\title{
Simulação computacional de escoamentos reativos com baixo número de Mach aplicando técnicas de refinamento adaptativo de malhas
}

\author{
Priscila Cardoso Calegari
}

TESE APRESENTADA

$\mathrm{AO}$

Instituto de Matemática e Estatística

DA

UNIVERSIDADE DE SÃo PAUlO

PARA

OBTENÇÃO DO TÍTULO

$\mathrm{DE}$

Doutor EM CiênCIAS

Área de Concentração: Matemática Aplicada
Orientador: Prof. Dr. Alexandre Megiorin Roma

Durante o desenvolvimento deste trabalho o autor recebeu auxílio financeiro do $\mathrm{CNPq}$ e da CAPES

São Paulo, agosto de 2012 



\title{
Simulação computacional de escoamentos reativos com baixo número de Mach aplicando técnicas de refinamento adaptativo de malhas
}

\author{
Priscila Cardoso Calegari
}

Esta versão da tese contém as correções e alterações sugeridas pela Comissão Julgadora durante a defesa da versão original do trabalho, realizada em 12/06/2012. Uma cópia da versão original está disponível no Instituto de Matemática e Estatística da Universidade de São Paulo.

Comissão Julgadora:

- Prof. Dr. Alexandre Megiorin Roma (orientador) - IME-USP

- Prof. Dr. Luis Carlos de Castro Santos - IME-USP

- Prof. Dr. Aristeu da Silveira Neto - UFU

- Prof. Dr. Ricardo Serfaty - PETROBRAS

- Prof. Dr. Antonio Castelo Filho - ICMC-USP 



\section{Agradecimentos}

Ao professor Alexandre pela paciência e disposição em me orientar, por sua luta diária em melhorar nosso ambiente de trabalho, por todo apoio, confiança e conselhos para o meu crescimento profissional e pessoal. Ao professor Guenther por apresentar o problema e por toda sua disposição e contribuição. Ao professor Luis Carlos por todas as sugestões e envolvimento na realização deste trabalho. Aos membros da banca por todas as sugestões e correções que enriqueceram o trabalho. Aos meus pais, por todo carinho e apoio ao longo destes onze anos que estive distante, amo vocês. À pessoa maravilhosa que acompanhou a realização deste trabalho diariamente: Álvaro, que sempre foi meu maior incentivador, companheiro de todas as horas, por todo o seu amor, envolvimento e compreensão. À toda minha família (em Santa Catarina e em Goiás), principalmente meus sobrinhos, por compreenderem minha ausência em suas comemorações. Aos colegas do LabMap que me acompanharam nesta jornada, Anderson, Daniel, Diane, Luciane, Marcelo, Nelson, Pedro e Tatiane. Em especial aos meus irmãos Wellington e Catalina. Ao Rudimar pela disposição em ler a versão preliminar. Ao Nils, ao Newton e ao Fernando, colegas engenheiros. Ao pessoal do MFLab, da Universidade Federal de Uberlândia, pela disposição em responder minhas dúvidas, em especial Millena, Pivello, Rafael, João Marcelo e Leonardo. Aos administradores do LabMap, Oda, Chico e Marcello por manterem nosso laboratório de pé. Aos meus amigos por me apoiarem. À todas as pessoas que de alguma maneira contribuiram com a realização deste trabalho. Agradeço ao IME pelo auxílio financeiro e à CAPES e ao CNPq pelo suporte financeiro durante a realização deste trabalho. 
"Embora ninguém possa voltar atrás e fazer um novo começo, qualquer um pode começar agora e fazer um novo fim."

Chico Xavier. 


\section{Resumo}

\section{Calegari, P. C. Simulação computacional de escoamentos reativos com baixo número}

de Mach aplicando técnicas de refinamento adaptativo de malhas. 2012. Tese (Doutorado) - Instituto de Matemática e Estatística, Universidade de São Paulo, 2012.

O foco principal do presente trabalho é estender uma metodologia numérica embasada no uso de uma técnica de refinamento adaptativo de malha (AMR - Adaptive Mesh Refinement) e no uso de esquemas temporais multipasso implícitos-explícitos (IMEX) a aplicações envolvendo escoamentos reativos com baixo número de Mach. Originalmente desenvolvida para escoamentos incompressíveis, a formulação euleriana daquela metodologia emprega as equações de Navier-Stokes como modelo matemático para descrever a dinâmica do escoamento e o Método da Projeção, baseado no divergente nulo da velocidade do escoamento, para tratar o acoplamento pressão-velocidade presente na formulação com variáveis primitivas. Tal formulação euleriana original é estendida para acomodar novas equações agregadas ao modelo matemático da fase contínua: conservação de massa, fração de mistura (para representar as concentrações de combustivel e oxidante), e energia. Além disso, uma equação termodinâmica de estado é integrada ao modelo matemático estendido e é empregada juntamente com a equação de conservação de massa para produzir uma nova restrição (não nula desta vez) ao divergente do campo de velocidade. Assume-se que o escoamento ocorre a baixo número de Mach (hipótese principal). O Método de Diferença Finita é empregado na discretização espacial das variáveis eulerianas de estado, empregando-se uma malha AMR. As vantagens e dificuldades desta extensão são cuidadosamente investigadas e reportadas. Pela importância, do ponto de vista de aplicações práticas, alguns estudos numéricos preliminares envolvendo escoamentos incompressíveis turbulentos com sprays são realizados (as gotículas compõem a fase dispersa). Num primeiro momento, apenas sprays com gotículas inertes são considerados. Embora ainda apenas iniciais, tais estudos já se mostram importantes pois identificam com clareza, em primeira instância, algumas das dificuldades inerentes a serem enfrentadas ao se tratar dentro desta nova metodologia um conjunto "relativamente grande" de gotículas lagrangianas. No caso de escoamentos incompressíveis turbulentos com sprays, a integração temporal se dá com métodos IMEX para a fase contínua e com o Método de Euler Modificado para a fase dispersa. A turbulência, em todos os casos que a envolvem, é tratada pelo modelo de Simulação das Grandes Escalas (LES - Large Eddy Simulation). As simulações computacionais se dão em um domínio tridimensional, um parelelepípedo, e empregam uma extensão (resultante do presente trabalho) do código "AMR3D", um programa de computador sequencial implementado em Fortran90, oriundo de uma colaboração de longa data entre o IME-USP e o MFLab/FEMEC-UFU (Laboratório de Dinâmica de Fluidos da Universidade Federal de Uberlândia). O processamento foi efetuado no LabMAP (Laboratório da Matemática Aplicada do IME-USP). 
Palavras-chave: AMR, IMEX, Método da Projeção, Escoamentos Reativos, Baixo Número de Mach, Sprays, Métodos Multinível-Multigrid. 


\section{Abstract}

\section{Calegari, P. C. Computational simulation of low Mach number reacting flows apply-}

ing adaptative mesh refinement techniques. 2012. Thesis (Ph.D.) - Institute of Mathematics and Statistics, University of São Paulo, 2012.

It is the main goal of the present work to extend a numerical methodology based on both the use of an adaptive mesh refinement technique (AMR) and the use of a multistep, implicit-explicit time-step strategy (IMEX) to applications involving low Mach number reactive flows. Originally developed for incompressible flows, the Eulerian formulation of that methodology employs the Navier-Stokes equations to model the flow dynamics and the Projection Method, based on the vanishing divergence of the velocity field, to tackle the pressure-velocity coupling present when using primitive variables. That Eulerian formulation is extended by adding a new set of equations to the original mathematical model, describing the various properties of the continuous phase: mass conservation, mixture fraction (to represent concentrations of fuel and oxidizer) and energy. Also, a thermodynamic equation of state is included into the extended mathematical model which is employed, along with the equation for the conservation of mass, to derive a new restriction (this time, different from zero) to the divergence of the velocity field. It is assumed that one is dealing with a low Mach number flow (the main hipothesis). The discretization in space employs the Finite Difference Method for the Eulerian variables on a AMR mesh. Advantages and difficulties of such an extension of the previous methodology are carefully investigated and reported. For its importance in the real-world applications, few preliminary numerical studies involving incompressible turbulent flows with sprays are performed (the droplets form what it is called the dispersed phase). Only sprays formed by inert droplets are considered. Even though initial yet, such studies are most important because they clearly identify, first hand, certain difficulties in handling relatively "large" sets of Lagrangian droplets in the context of this new AMR methodology. In the context of turbulent incompressible flows with sprays, the overall time-step scheme is given by IMEX methods for the continuous phase and by the Improved Euler Method for the dispersed phase. In all the cases in which it is considered, turbulence is modeled by the Large Eddy Simulation (LES) model. The computational simulations are held in a tridimensional domain given by a paralellepiped and all of them employ the extention (resulting of the present work) of the "AMR3D" code, a sequencial computer program implemented in Fortran90, whose origin is the collaborative work between IMEUSP and MFLab/FEMEC-UFU (Fluid Dynamics Laboratory, Federal University of Uberlândia). Computations were performed at LabMAP (Applied Mathematics Laboratoy at IME-USP).

Keywords: AMR, IMEX, Projection Method, Reacting Flows, Low Mach Number, Sprays, Multilevel-Multigrid Methods. 


\section{Sumário}

Lista de Figuras $\quad$ xi

$\begin{array}{ll}\text { Lista de Tabelas } & \text { XV }\end{array}$

1 Introdução $\quad \mathbf{1}$

1.1 Motivação e objetivos . . . . . . . . . . . . . . . . . . . . . . 2

1.2 Escoamentos reativos . . . . . . . . . . . . . . . . . . . . 3

1.3 Método da projeção para escoamentos reativos com baixo número de Mach . . . . 4

1.4 Refinamento adaptativo de malhas . . . . . . . . . . . . . . . . 4

1.5 Organização do trabalho . . . . . . . . . . . . . . . . . . . . . . 6

2 Modelo matemático $\quad 7$

2.1 Equações para um escoamento reativo na fase contínua . . . . . . . . . . . . 7

2.1.1 Equação da conservação da massa . . . . . . . . . . . . . . . . . 8

2.1.2 Equação da quantidade de movimento linear . . . . . . . . . . . . . 8

2.1.3 Equação da conservação da fração mássica das espécies químicas . . . . . . . 9

2.1.4 Equação da conservação da energia . . . . . . . . . . . . . . . . . . . 10

2.1.5 Equação do estado termodinâmico . . . . . . . . . . . . . . . . . . 12

2.1.6 Equações adimensionalizadas . . . . . . . . . . . . . . . . . 12

2.2 Escoamentos reativos com baixo número de Mach . . . . . . . . . . . . . . . . 13

2.3 Modelo da reação química . . . . . . . . . . . . . . . . . . . . 15

2.4 Modelo matemático para a fase dispersa de um escoamento incompressível . . . . . 18

2.4.1 Equações do movimento das gotículas . . . . . . . . . . . . . . . . . . . 19

2.5 Acoplamento entre as fases . . . . . . . . . . . . . . . . . . . . . 20

2.6 Sumário dos modelos matemáticos . . . . . . . . . . . . . . . . . 21

2.6.1 Modelo matemático para um escoamento incompressível (não-reativo) . . . 21

2.6.2 Modelo matemático para um escoamento incompressível (não-reativo) com gotículas inertes . . . . . . . . . . . . . . . . . . 21

2.6.3 Modelo matemático para um escoamento reativo com baixo número de Mach 22

2.7 Nomenclatura . . . . . . . . . . . . . . . . . . . . . . . . . . . 23

3 Metodologia numérica $\mathbf{2 5}$

3.1 Método da Projeção . . . . . . . . . . . . . . . . . . . . . . 25

3.1.1 Extensão do método da projeção para escoamentos reativos com baixo número de Mach . . . . . . . . . . . . . . . . . . . . . . . 27 
3.2 Solução das equações que modelam a fase contínua . . . . . . . . . . . . . . . . 28

3.2.1 Discretização temporal . . . . . . . . . . . . . . . . . . . . . . 28

3.2.2 Estratégia semi-implícita . . . . . . . . . . . . . . . . . . . 29

3.2.3 Discretização do domínio computacional . . . . . . . . . . . . . . . . . 30

3.2.4 Discretização espacial . . . . . . . . . . . . . . . . . . . . . . . . . 31

3.2.5 Método Multigrid. . . . . . . . . . . . . . . . . . . . . . . 34

3.2.6 Sumário da metodologia numérica para a fase contínua . . . . . . . . . . . . 36

3.3 Solução das equações que modelam a fase dispersa . . . . . . . . . . . . . . . . . 39

3.3.1 Discretização temporal da fase dispersa . . . . . . . . . . . . . . . . . . . 40

3.3.2 Operadores de interpolação entre as fases contínua e dispersa . . . . . . . . 40

3.3.3 Sumário da metodologia numérica para um escoamento incompressível contendo gotículas . . . . . . . . . . . . . . . . . . . 42

3.4 Nomenclatura . . . . . . . . . . . . . . . . . . . . . . . 43

4 Malha adaptativa bloco-estruturada $4 \mathbf{4 5}$

4.1 Descrição de uma malha bloco-estruturada . . . . . . . . . . . . . . . . . . . 45

4.2 Geração de uma malha bloco-estruturada . . . . . . . . . . . . . . . . . . . . . 48

4.2.1 Malha inicial do domínio computacional . . . . . . . . . . . . . . . . . 49

4.2.2 Seleção de células computacionais para refinamento . . . . . . . . . . . . 50

4.2.3 Localização das gotículas na malha bloco-estruturada . . . . . . . . . . . 50

4.3 Células computacionais fantasmas . . . . . . . . . . . . . . . . . . . . . 51

4.4 Método Multigrid-Multinível . . . . . . . . . . . . . . . . . . . . . . 52

4.5 Sumário da metodologia numérica na malha bloco-estruturada . . . . . . . . . 53

4.6 Nomenclatura . . . . . . . . . . . . . . . . . . . . . 54

5 Verificação da metodologia numérica $\mathbf{5 5}$

5.1 Verificação numérica por meio de soluções manufaturadas . . . . . . . . . . . . . 55

5.2 Escoamento incompressível . . . . . . . . . . . . . . . . . . . . 57

5.3 Advecção-difusão de uma grandeza escalar . . . . . . . . . . . . . . . . . . . . 59

5.4 Escoamento reativo com baixo número de Mach . . . . . . . . . . . . . . . 60

5.5 Fase dispersa . . . . . . . . . . . . . . . . . . . . . 61

5.6 Nomenclatura . . . . . . . . . . . . . . . . . . . 63

6 Resultados numéricos $\quad \mathbf{6 5}$

6.1 Simulação de escoamentos incompressíveis (não-reativos) . . . . . . . . . . . 65

6.1.1 Escoamento incompressível (não-reativo) laminar . . . . . . . . . . . . . 66

6.1.2 Escoamento incompressível (não-reativo) turbulento . . . . . . . . . . . . 67

6.2 Simulação de um escoamento (não-reativo) com gotículas inertes . . . . . . . . . 77

6.3 Simulação de escoamentos reativos a baixo Mach . . . . . . . . . . . . . . . . 83

6.3.1 Escoamento reativo laminar . . . . . . . . . . . . . . . . . . 84

6.3.2 Escoamento reativo turbulento ................. 86

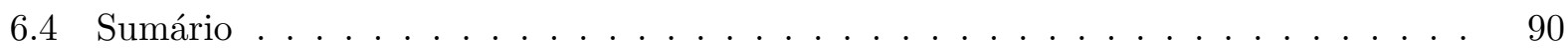

6.5 Nomenclatura . . . . . . . . . . . . . . . . . . . . 93 
7 Considerações finais $\quad 95$

7.1 Contribuições . . . . . . . . . . . . . . . . . . . . . . 97

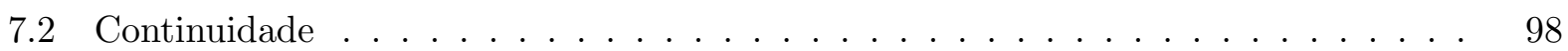

A Modelo de turbulência $\quad 99$

A.1 Modelo de turbulência para a taxa de reação . . . . . . . . . . . . . . . . . . 101

A.2 Modelo da fração de mistura em escoamentos turbulentos . . . . . . . . . . . . 102

B Sistema de equações rígido $\quad 105$

$\begin{array}{llr}\text { C } & \text { Dedução das formas da equação da energia } & 107\end{array}$

D Adimensionalização das equações $\quad 111$

D.1 Equação da conservação da massa adimesional . . . . . . . . . . . . . . . . 111

D.2 Equação da conservação das espécies químicas adimensional . . . . . . . . . . . . . 112

D.3 Equação da quantidade de movimento linear adimensional . . . . . . . . . . . . . 112

D.4 Equação da temperatura adimensional . . . . . . . . . . . . . . . . . . . . 113

D.5 Equação do estado termodinâmico adimensional . . . . . . . . . . . . . . . . . . . . 114

$\begin{array}{lr}\text { Referências Bibliográficas } & 117\end{array}$

$\begin{array}{lr}\text { Índice Remissivo } & 123\end{array}$ 


\section{Lista de Figuras}

1.1 Entrada do combustível e do oxidante na região de reação. A reação química em (a) resulta em uma chama pré-misturada e em (b) em uma chama não pré-misturada.

1.2 Corte central no domínio computacional tridimensional. (a) Componente da velocidade na direção do escoamento junto com a malha bloco-estruturada com refinamento adaptativo gerada pela técnica AMR. (b) Blocos que compõem a malha bloco-estruturada com refinamento adaptativo. . . . . . . . . . . . .

3.1 Localização das variáveis escalares e vetoriais discretizadas em uma célula computacional. (a) Variáveis escalares no centro de uma célula computacional. (b) Componente da velocidade na direção $x_{1}$ no centro da face $x_{2} x_{3}$. (c) Componente da velocidade na direção $x_{2}$ no centro da face $x_{1} x_{3}$. (d) Componente da velocidade

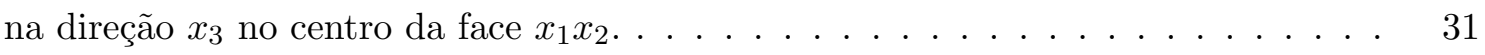

3.2 Aproximações quadráticas utilizadas pelo esquema QUICK em uma dimensão. . . 33

3.3 Restrição para uma variável escalar calculada no centro de uma célula computacional. 35

3.4 Prolongamento para uma variável calculada no centro da célula computacional. . . 36

3.5 Localização de uma variável escalar $\phi$ e uma vetorial $\mathbf{u}=\left(u_{1}, u_{2}, u_{3}\right)$ discretizadas, respectivamente no centro e nas faces de uma célula computacional e as gotículas lagrangianas, representadas pelo símbolo $\bullet$, contidas nesta célula. . . . . . . . . .

4.1 Malha bloco-estruturada em um domínio computacional $[0,1] \times[0,1] \times[0,1]$ com dois níveis de refinamento: (a) malha tri-dimensional, (b) corte na direção $x_{1}=0.42$, (c) corte na direção $x_{3}=0.45$ e (d) corte na direção $x_{2}=0.3$, Nós (2007) . . . . . . .

4.2 Elementos de uma malha bloco-estruturada bidimensional: (a) malha base; (b)-(d) regiões cobertas por um nível de refinamento; (c) nível de refinamento com dois blocos discretizados; (e) nível de refinamento com três blocos discretizados e (f) malha bloco-estruturada. . . . . . . . . . . . . . . . .

4.6 Exemplo de região de segurança bidimensional. A região de segurança está contornada por uma linha tracejada. . . . . . . . . . . . . . . 50

4.7 Lista encadeada. . . . . . . . . . . . . . . . . . . . 50

5.1 Malha composta utilizada na verificação numérica: (a) malha 3D no formato "L", (b) corte no domínio computacional na direção $x_{1}$, (c)-(d) cortes no domínio computacional na direção $x_{2}$ e (e)-(f) cortes no domínio computacional na direção $x_{3}$

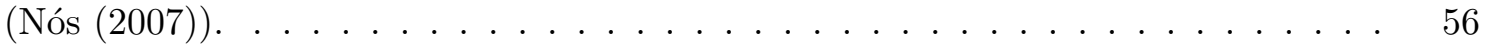

6.1 Esboço da entrada da componente da velocidade na direção do escoamento. . . . . 66 
6.3 Zoom da velocidade do escoamento em um corte central e malha bloco-estruturada .

6.2 Escoamento incompressível (não-reativo) laminar: componente da velocidade na direção do escoamento em um plano de corte no domínio computacional em $x_{3}=$ $8 \times 10^{-2} \mathrm{~m}$ em diferentes instantes de tempo. . . . . . . . . . . .

6.4 Decaimento da velocidade na direção do escoamento na linha de centro do domínio

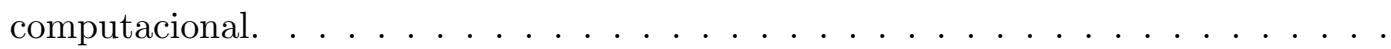

6.5 Razão entre os tempos gastos em uma malha uniforme (TMU) e em uma malha composta bloco-estruturada (TMC) . . . . . . . . . . . . . . .

6.6 Malha tridimensional com refinamento adaptativo. . . . . . . . . . . . . .

6.7 Escoamento incompressível (não-reativo) turbulento: componente da velocidade na direção do escoamento em um plano de corte no centro do domínio computacional

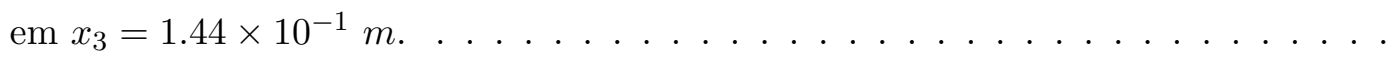

6.8 Velocidade do jato na direção do escoamento com a malha bloco-estruturada em um plano de corte central. . . . . . . . . . . . . . . . .

6.9 Escoamento incompressível (não-reativo) turbulento: componente da velocidade na direção do escoamento em um instante de tempo em um plano de corte transversal

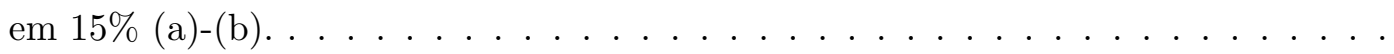

6.10 Escoamento incompressível (não-reativo) turbulento: componente da velocidade na direção do escoamento em um instante de tempo em em planos de corte transversais em $25 \%$ (a)-(b), $50 \%$ (c)-(d) e $75 \%$ (e)-(f) . . . . . . . . . . . . . .

6.12 Decaimento médio da componente da velocidade na direção do escoamento na linha de centro do domínio computacional. . . . . . . . . . . . . .

6.15 Esboço da entrada das gotículas e da componente da velocidade na direção do es-

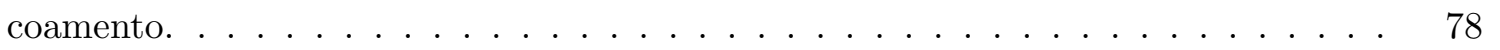

6.16 Distribuição uniforme sobre um disco. . . . . . . . . . . . . . . . . . . . 79

6.17 Distribuição log-normal para o diâmetro das gotículas. . . . . . . . . . . . . . 80

6.18 Velocidade na direção do escoamento e malha bloco-estruturada gerada na simulação do escoamento com gotículas em um corte transversal. . . . . . . . . . . . . . .

6.19 Escoamento incompressível (não-reativo) turbulento com gotículas inertes: (a)-(d) velocidade das gotículas na direção do escoamento com um plano de corte central.

6.20 Escoamento incompressível (não-reativo) turbulento com gotículas inertes: (a)-(b) velocidade das gotículas na direção do escoamento em um plano de corte central. .

6.21 Perfil da velocidade das gotículas na linha radial. . . . . . . . . . . . . .

6.22 Esboço da entrada do combustível e do ar na direção do escoamento reativo. . . .

6.23 Escoamento reativo laminar: temperatura do escoamento e malhas adaptativas em um plano de corte em $x_{3}=5 \times 10^{-2} \mathrm{~m} \ldots \ldots \ldots \ldots \ldots$

6.24 Temperatura do escoamento e malha bloco-estruturada gerada, em corte transversal na metade do domínio computacional. . . . . . . . . . . . . . .

6.25 Escoamento reativo turbulento: Fração de mistura em um plano de corte no centro do domínio computacional em $x_{3}=1.44 \times 10^{-1} \mathrm{~m} \ldots \ldots \ldots \ldots$. . . . . .

6.26 Escoamento reativo turbulento: Temperatura do escoamento em um plano de corte no centro do domínio computacional em $x_{3}=1.44 \times 10^{-1} \mathrm{~m}$. . . . . . . . . . 
6.27 Decaimento na linha de centro da velocidade média na direção do escoamento e dados experimentais. . . . . . . . . . . . . . . . . . 90

6.28 Decaimento na linha de centro na direção do escoamento da fração demistura média e dados experimentais. . . . . . . . . . . . . . . . . .

6.29 Perfil da temperatura na linha de centro na direção do escoamento e dados experimentais. . . . . . . . . . . . . . . . . . . 


\section{Lista de Tabelas}

2.1 Propriedades de referência do escoamento e suas unidades $($ SI) . . . . . . . . . . . 13

5.1 Erro global e ordem de aproximação para o campo de velocidade e pressão na malha

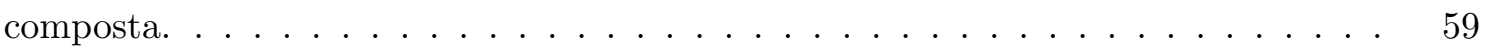

5.2 Erro e ordem de aproximação para a equação do transporte (5.18) . . . . . . . . . . 59

5.3 Erro e ordem de aproximação do campo de velocidades, da pressão, da densidade e da fração de mistura na malha composta. . . . . . . . . . . . . . . . . 61

5.4 Erro e ordem de aproximação das posições das gotículas. . . . . . . . . . . . . . . . 62

5.5 Erro e taxa de convergência da interpolação do campo de velocidade do escoamento

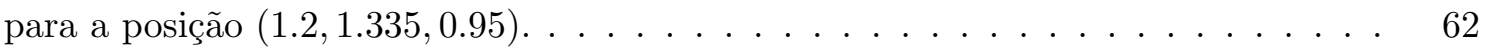

6.1 Número de células computacionais em diferentes malhas. . . . . . . . . . . . . . 77

6.2 Temperatura máxima da chama laminar. . . . . . . . . . . . . . . . . . 86 


\section{Lista de Algoritmos}

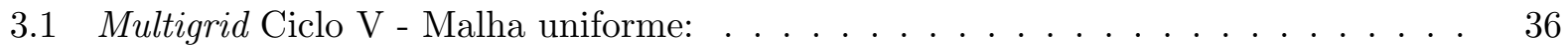

3.2 Um passo de integração - Escoamento reativo com baixo número de Mach: . . . . . 38

3.3 Um passo de integração - Escoamento incompressível (não-reativo) : . . . . . . . . . 39

3.4 Um passo de integração - Escoamento incompressível (não-reativo) com gotículas: . 42

4.1 Multigrid-Multinível Ciclo V - Malha bloco-estruturada: . . . . . . . . . . . . . . 52

4.2 Um passo de integração na malha bloco-estruturada - Escoamento incompressível (não-reativo) com gotículas: . . . . . . . . . . . . . . . . . . . 53 


\section{Capítulo 1}

\section{Introdução}

A combustão é uma importante área de pesquisa da engenharia que envolve diversos fenômenos como turbulência, transferência de calor, reações químicas, radiação, entre outros processos químicos e físicos. Dentre os processos de combustão estão aqueles que, devido a emissão de gases poluentes, são altamente nocivos. Desta maneira, modelos matemáticos são empregados para predizer escoamentos, temperaturas, concentrações de espécies químicas (resultantes das reações químicas), com o objetivo de minimizar efeitos nocivos ao meio ambiente. Neste contexto, é crescente o interesse em simulações numéricas de escoamentos reativos com combustíveis renováveis e menos agressivos ao meio ambiente como o etanol.

Uma das intenções do presente trabalho é investigar o desempenho e a aplicabilidade de técnicas de refinamento adaptativo de malhas visando a simulação numérica de escoamentos reativos com baixo número de Mach e escoamentos incompressíveis com gotículas inertes sem evaporação. Um grupo de pesquisadores do Instituto de Matemática e Estatística (IME-USP) e da Faculdade de Engenharia Mecânica da Universidade Federal de Uberlândia (MFLab/FEMEC-UFU) vem desenvolvendo um código computacional denominado "AMR3D" (veja Nós (2007) e Ceniceros et al. (2010a)). A metodologia empregada no código AMR3D usa técnicas de refinamento adaptativo de malhas e o Método da Projeção de Chorin-Temam para simular escoamentos incompressíveis. Originalmente, o código AMR3D foi desenvolvido por Nós (2007). Atualmente, o código vem sendo ampliado em parceria com o MFLab-UFU. Alguns trabalhos fizeram uso desta metodologia como Roma et al. (1999), Ceniceros et al. (2010a) e Ceniceros et al. (2010b). Observando a eficiência da metodologia numérica em desenvolvimento (quando aplicada a escoamentos incompressíveis), foi decidido estendê-la a escoamentos reativos com baixo número de Mach. A extensão proposta parte de três componentes principais: uma técnica de refinamento adaptativo de malhas para discretizar o domínio computacional (Berger e Oliger (1984) e Berger e Colella (1989)), a utilização de esquemas implícitos-explícitos para a discretização temporal (Ascher et al. (1995) e Wang e Ruuth (2008)) e a utilização de um método da projeção para tratar o acoplamento pressão-velocidade (Nós (2007), Ceniceros et al. (2010b) e Roma et al. (1999)).

Este capítulo apresenta, de uma forma geral, os principais tópicos deste trabalho. A Seção 1.2 apresenta uma breve descrição do problema. A Seção 1.3 introduz a extensão do método da Projeção de Chorin-Temam para escoamentos com baixo número de Mach. A Seção 1.4 apresenta o potencial da técnica AMR na simulação numérica de problemas de combustão. Na Seção 1.1, são descritos os objetivos do presente trabalho e, na Seção 1.5, é descrita a estrutura do trabalho. 


\subsection{Motivação e objetivos}

A simulação numérica de problemas que envolvem reações químicas e escoamentos turbulentos, necessita de boa resolução para capturar grande parte dos fenômenos envolvidos, Pember et al. (1998), Pitsch e Steiner (2000), García (2009), Apte et al. (2009), Day e Bell (2000), Gao e Groth (2008). O uso de malhas refinadas é um ponto favorável para a resolução de escoamentos reativos. No presente trabalho aplica-se a estratégia AMR, (Adaptive Mesh Refinement), proposta por Berger e Oliger (1984), para a geração de malhas bloco-estruturadas com refinamento adaptativo. Além disso, aplica-se uma extensão do Método da Projeção para tratar o acoplamento pressãovelocidade, baseada na proposta de Pember et al. (1998), para a solução de um escoamento reativo com baixo número de Mach. A extensão proposta no presente trabalho, parte de três componentes principais: uma técnica de refinamento adaptativo de malhas para discretizar o domínio computacional, a utilização de esquemas implícitos-explícitos (IMEX, veja Ascher et al. (1995) e Wang e Ruuth (2008)) para a discretização temporal e do Método da Projeção para tratar o acoplamento pressão-velocidade. Nas próximas seções cada uma destas componentes são descritas brevemente. A partir destas componentes foram desenvolvidas:

- uma extensão do Método da Projeção de Chorin-Temam para escoamentos reativos com baixo número de Mach combinado ao modelo químico baseado na fração de mistura;

- uma implementação da equação do transporte de uma variável escalar; e

- uma análise de critérios de refinamento adaptativos para captar os fenômenos físicos envolvidos no problema estudado.

Visando uma simulação numérica de um escoamento incompressível (não-reativo) com gotículas inertes sem evaporação adotou-se a formulação lagrangiana. A partir do código computacional com a metodologia numérica para um escoamento incompressível (não-reativo), foi necessário:

- adicionar estruturas de dados na ferramenta numérica para a descrição das gotículas; e

- incluir métodos numéricos para a solução de um sistema de equações diferenciais ordinárias associado a cada gotícula.

- investigar distribuições de probabilidade para o tamanho, posição e velocidade das gotículas; e

- análisar critérios de refinamento adaptativos para captar os fenômenos físicos envolvidos no problema estudado.

O presente trabalho trouxe informações relevantes associadas à metodologia numérica em uso. Pela primeira vez, o código AMR3D foi aplicado a escoamentos de jatos. Com isso, foi possível identificar dificuldades não encontradas antes como, por exemplo, o uso abusivo de células fantasmas que levam a um alto custo computacional. Algumas medidas que evitam um grande número células fantasmas foram tomadas no presente trabalho, mostrando-se eficazes.

Uma grande energia do grupo IME-MFLab vem sendo empregada para a aceleração do tempo computacional gasto pelo código, tanto na paralelização via decomposição de domínios quanto 
na montagem da matriz associada ao problema. O intuito da montagem da matriz é resolver os sistemas lineares oriundos, das discretizações espaciais e temporais, utilizando bibliotecas com métodos já paralelizados.

\subsection{Escoamentos reativos}

As chamas resultantes dos processos de combustão são, em geral, classificadas em pré-misturadas e não pré-misturadas. Nas chamas pré-misturadas, combustível e oxidante entram na região de reação já misturados. Nas chamas não pré-misturadas combustível e oxidante entram separadamente na região de reação (veja Turns (1996) e Poinsot e Veynante (2005)). A Figura 1.1 mostra a entrada do combustível e do oxidante que caracterizam os dois tipos de chama.

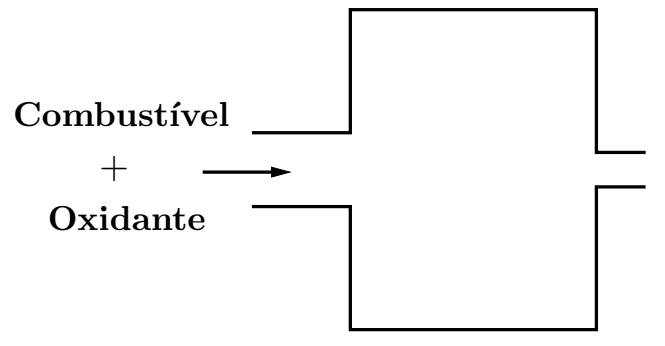

(a)

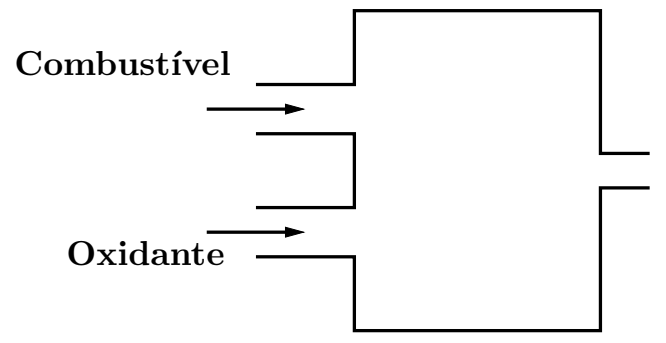

(b)

Figura 1.1: Entrada do combustível e do oxidante na região de reação. A reação química em (a) resulta em uma chama pré-misturada e em (b) em uma chama não pré-misturada.

Na chama não pré-misturada, as espécies químicas são determinadas pela resolução de um sistema de equações diferenciais. Este sistema pode envolver centenas de espécies e reações químicas (veja Pember et al. (1998), Day e Bell (2000), Najm e Knio (2005), Gao e Groth (2008)). Alguns modelos para a reação química contornam a dificuldade de se resolver este sistema, assumindo um modelo simplificado com um número menor de espécies químicas, (veja Kuo (2005), Versteeg e Malalasekera (2007), Turns (1996), Apte et al. (2009), Pierce e Moin (2004)). No presente trabalho adotou-se o emprego do modelo químico que assume a reação química infinitamente rápida e estequiométrica. Este modelo considera a natureza global da reação química por meio de uma única equação de conservação para uma variável escalar denominada fração de mistura (veja Kuo (2005), Turns (1996) e Poinsot e Veynante (2005)). A aplicação da metodologia proposta é baseada na simulação numérica de uma chama de difusão (Chama D). Com isso, espera-se explorar as vantagens da metodologia numérica e possíveis dificuldades a serem encontradas.

O processo da queima do combustível líquido na forma de spray é considerado um escoamento bifásico e pode ser modelado de duas maneiras: por intermédio de uma fomulação eulerianaeuleriana, onde cada fase é localizada em uma célula computacional por meio de uma função indicadora, (veja Crowe et al. (1998), García (2009) e Sirignano (1999)), ou por intermédio de uma formulação euleriana-lagrangiana, a qual possui dois conjuntos de equações acoplados, um para a fase gasosa, também chamada de fase contínua e outro para a fase líquida, também chamada de fase dispersa, representada por gotículas, (veja e Sirignano (1999), García (2009), Apte et al. (2009) e Crowe et al. (1998)). No presente trabalho, é empregada uma formulação eulerianalagrangiana. A fase contínua é modelada por meio das equações da conservação da massa, da conservação da fração de mistura, da conservação da energia e da quantidade de movimento. A 
fase dispersa, composta pelas gotículas é modelada por meio de um sistema de equações diferenciais ordinárias que descreve a evolução da posição, da temperatura, da massa e da velocidade de cada gotícula. O presente trabalho investiga a aplicabilidade da metodologia numérica a escoamentos incompressíveis (não-reativos) com gotículas. Serão consideradas gotículas inertes sem evaporação conforme Stårner e Masri (2003) e De et al. (2011) visando, futuramente, a simulação de um escoamento reativo com baixo número de Mach e com gotículas.

\subsection{Método da projeção para escoamentos reativos com baixo número de Mach}

Nas aplicações de escoamentos incompressíveis estudadas pelo grupo IME-MFLab, utiliza-se o método da Projeção de Chorin-Temam para tratar o acoplamento pressão-velocidade nas equações de Navier-Stokes (Roma et al. (1999), Nós (2007), Ceniceros et al. (2010a), Ceniceros et al. (2010b)). O método da projeção para escoamentos reativos com baixo número de Mach, o qual trata o acoplamento pressão-velocidade, é baseado na expansão assintótica das equações de Navier-Stokes em termos do número de Mach. A expansão, proposta por Majda e Sethian (1985) e Rehm e Baum (1978), motivou Pember et al. (1998) a estender um método da projeção para escoamentos reativos com baixo número de Mach. Neste caso, uma restrição para o divergente do campo de velocidade (não nula) é obtida a partir da equação do estado termodinâmico e da equação da conservação de massa.

A versão estendida do método da projeção para escoamentos com baixo número de Mach é utilizada por Bell (2005), Day e Bell (2000) e Nicoud (2000), que utilizam um modelo químico detalhado. No presente trabalho uma restrição para o divergente do campo de velocidade é determinada para o modelo químico baseado na fração de mistura, sendo esta abordagem, do nosso conhecimento, inédita no contexto da metodologia numérica adotada.

\subsection{Refinamento adaptativo de malhas}

A adaptatividade é uma técnica importante a ser considerada para a solução eficiente de equações diferenciais parciais. O termo refinamento adaptativo de malhas (ou simplesmente AMR) engloba uma coleção de aproximações aplicáveis a uma variadade de problemas, conforme descreve Plewa et al. (2005). Entretanto, a ideia ainda é a mesma: concentrar poder computacional onde é mais necessário com o aumento da resolução em regiões de interesse. Esta ideia evita o alto custo computacional necessário para simular um problema em uma malha uniforme tridimensional. Neste trabalho emprega-se a estratégia de refinamento adaptativo de malhas (AMR) proposta por Berger e Oliger (1984), Berger e Colella (1989) e Berger e Rigoutsos (1991).

A Figura 1.2 foi obtida aplicando a técnica AMR na simulação de um jato incompressível nãoreativo turbulento. Ela mostra um plano de corte central de uma malha bloco-estruturada com refinamento adaptativo contendo uma malha base e mais três níveis de refinamento. A Figura 1.2(a) mostra a componente da velocidade na direção do escoamento de um jato turbulento não-reativo e a Figura 1.2(b), os blocos discretizados que compõem a malha com refinamento adaptativo. Note que a união dos blocos discretizados com mesmo espaçamento formam um nível de refinamento e a união dos quatro níveis de refinamento (neste caso) compõem a malha bloco-estruturada. 


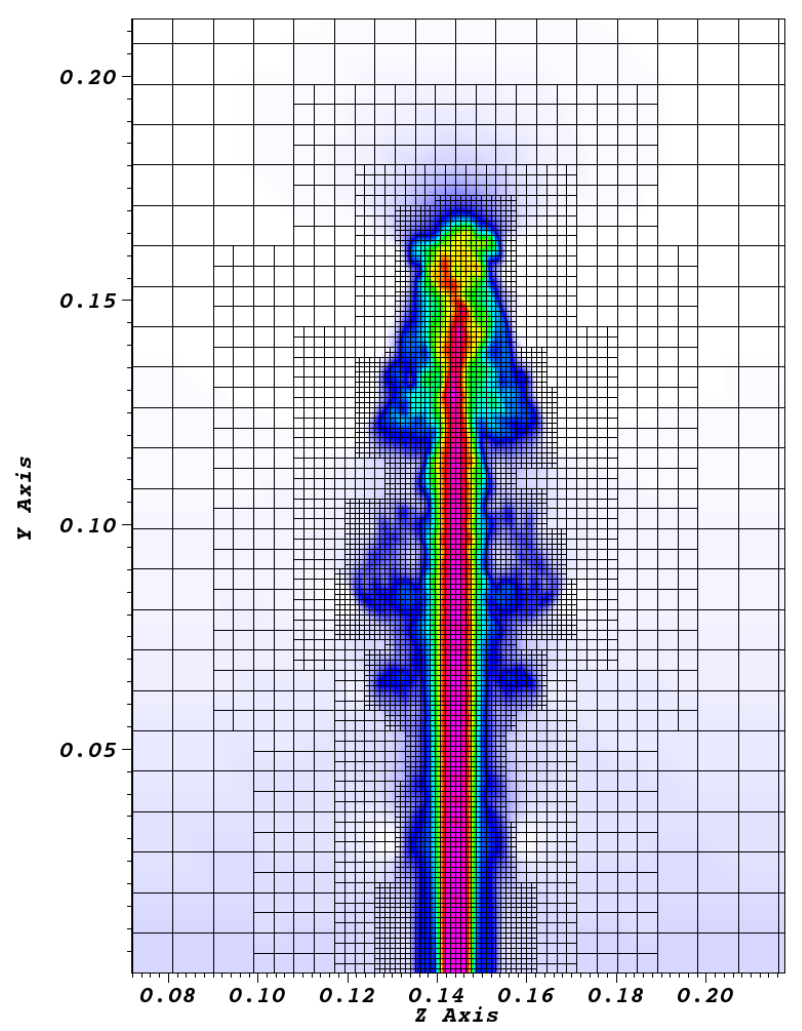

(a)

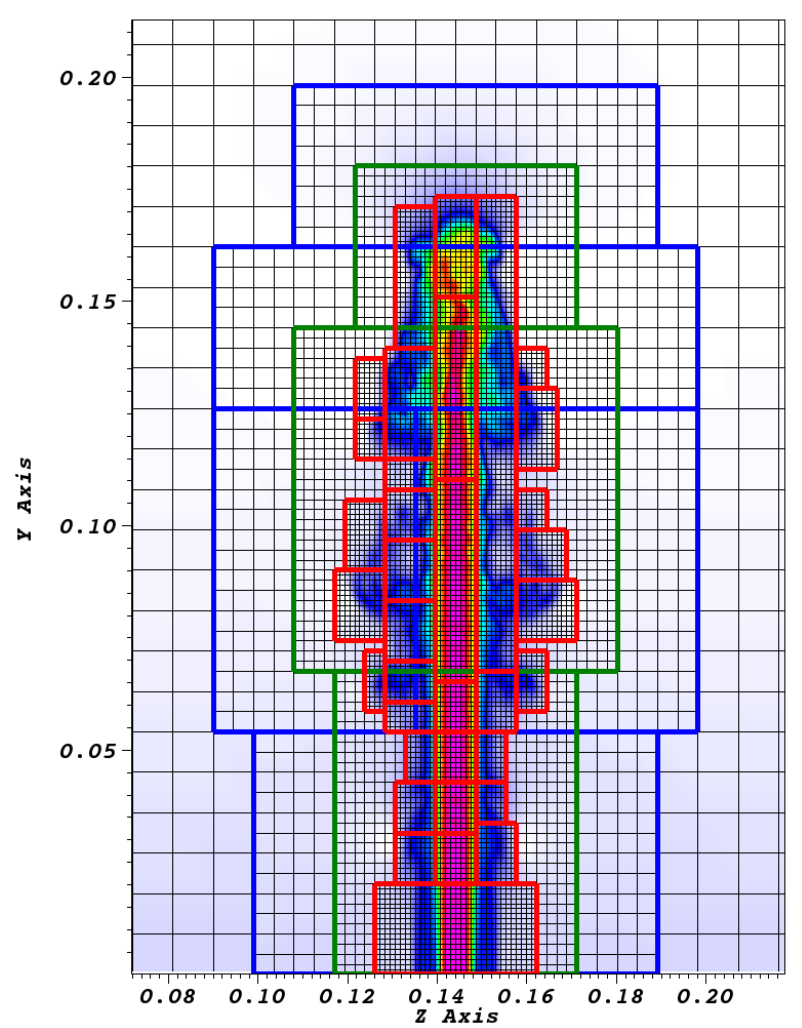

(b)

Figura 1.2: Corte central no domínio computacional tridimensional. (a) Componente da velocidade na direção do escoamento junto com a malha bloco-estruturada com refinamento adaptativo gerada pela técnica AMR. (b) Blocos que compõem a malha bloco-estruturada com refinamento adaptativo.

Na malha bloco-estruturada, as variáveis são dispostas em uma malha deslocada (modelo MAC, marker and cell): escalares no centro da célula computacional (por exemplo, massa específica, fração de mistura e viscosidade), e as componentes do campo de velocidade nas faces da célula computacional. Esquemas de diferenças finitas de segunda ordem são empregados na discretização dos operadores diferenciais espaciais. Para a discretização temporal são empregados os esquemas IMEX (Implicit-Explicit) de segunda ordem, os quais tratam o termo difusivo implicitamente e o termo advectivo explicitamente. O tratamento explícito do termo advectivo evita a resolução de um sistema não linear e o tratamento implícito do termo difusivo evita a escolha de um tamanho de passo de integração $\Delta t$ muito restritivo.

Na área de combustão a estratégia AMR vem sendo utilizada por Pember et al. (1998), Bell (2005), Day e Bell (2000), Northrup e Groth (2009) e Gao e Groth (2008) na simulação de chamas não pré-misturadas, de chamas de hidrogênio pré-misturadas, de chamas nucleares e problemas da área de astrofísica Zingale et al. (2009) e Nonaka et al. (2012). Além da estratégia AMR a metodologia destes trabalhos envolve aproximações a baixo número de Mach, modelo de química detalhada para as reações químicas, paralelismo e simulação numérica direta (DNS - Direct numerical simulation).

Os recentes trabalhos de simulação numérica de escoamento com gotículas (sprays) utilizam malhas não estruturadas (veja García (2009), Bini e Jones (2009), De et al. (2011) e Chrigui et al. 
(2012)). A simulação numérica de um escoamento com gotículas em uma malha bloco-estruturada com refinamento adaptativo, além da eficiência e precisão em regiões de interesse, facilita a localização das gotículas na malha. O uso da estratégia AMR para a simulação de escoamentos com uma nuvem de gotículas (spray), dentro do nosso conhecimento, possui seu grau de originalidade.

\subsection{Organização do trabalho}

No presente trabalho, a combinação de uma técnica AMR com um método implícito-explícito e um método da projeção é aplicada para resolver as equações que modelam o escoamento de dois jatos incompressíveis (não-reativos). Além disso, o trabalho apresenta uma metodologia numérica para a simulação de escoamentos incompressíveis (não-reativos) com gotículas inertes (Stårner e Masri (2003) e De et al. (2011)) e uma versão estendida do método da projeção visando a simulação de escoamentos reativos com baixo número de Mach.

O presente trabalho está estruturado como segue. O Capítulo 2 apresenta três modelos matemáticos: para um escoamento incompressível (não-reativo), para um escoamento incompressível (nãoreativos) com gotículas inertes e para um escoamento reativo com baixo número de Mach. Além disso, é descrito um modelo da reação química baseado na fração de mistura (escalar conservado). No Capítulo 3, é apresentada uma metodologia numérica para a simulação de um escoamento reativo com baixo número de Mach. Isso inclui uma extensão do método da projeção, uma discretização temporal para a fase contínua e uma discretização espacial. À metodologia numérica para escoamentos incompressíveis (não-reativos) é acrescentada à discretização temporal para a fase dispersa e os operadores responsáveis pelo acoplamento entre as fases. Com isso, estende-se à metodologia numérica a escoamentos incompressíveis (não reativos) com gotículas inertes sem evaporação. O Capítulo 4 apresenta a estratégia AMR e o método Multigrid-Multinível para a solução do sistema linear derivado da discretização da equação da conservação da fração de mistura. No Capítulo 5, são apresentadas as verificações numéricas da metodologia proposta por meio de soluções manufaturadas. No Capítulo 6, são apresentados os resultados numéricos das simulações dos jatos incompressíveis (não-reativos). Além disso, são apresentados resultados preliminares que mostram o potencial da técnica AMR, quando aplicada a um escoamento incompressível (nãoreativo) com gotículas inertes sem evaporação e a um escoamento reativo com baixo número de Mach (Chama D). O Capítulo 7 apresenta as considerações finais. A revisão da literatura é feita durante a apresentação de cada capítulo. No final dos Capítulos $2-6$ é apresentada uma lista de símbolos utilizados nos mesmos. Os Apêndices trazem informações que complementam o trabalho. 


\section{Capítulo 2}

\section{Modelo matemático}

Muitos problemas de combustão são modelados como escoamentos bifásicos onde se tem uma fase líquida (que pode ser modelada como gotículas de combustível) e uma fase gasosa (a qual modela o oxidante). A fase gasosa é denominada fase contínua e a fase líquida é denominada fase dispersa. No presente trabalho é empregada uma formulação matemática para um escoamento reativo na fase contínua, baseada nas equações de Navier-Stokes para um escoamento reativo (veja Kuo (2005), Versteeg e Malalasekera (2007), Turns (1996) e Poinsot e Veynante (2005)). Além disso, o presente trabalho emprega uma formulação matemática para a fase dispersa, a qual modela o movimento das gotículas inertes no escoamento (veja Stårner e Masri (2003), Crowe et al. (1998), García (2009) e De et al. (2011)). No presente trabalho a evaporação das gotículas na fase dispersa não é tratada.

Na Seção 2.1 são descritas as equações que modelam um escoamento reativo multicomponente da fase contínua. Na Seção 2.2 são descritas as equações que modelam um escoamento reativo com baixo número de Mach conforme descrevem Pember et al. (1998), Bell (2005), Day e Bell (2000) e Nicoud (2000). Estas equações são baseadas nas expansões assintóticas das equações de NavierStokes em termos do número de Mach apresentadas por Majda e Sethian (1985) e Rehm e Baum (1978). Na Seção 2.3 é descrito um modelo para as reações químicas que ocorrem na fase contínua. Este modelo é baseado na estrutura da chama de Burke-Schumann o qual assume que a reação química é infinitamente rápida e estequiométrica (Kuo (2005), Versteeg e Malalasekera (2007) e Poinsot e Veynante (2005)). Esta abordagem considera a natureza global da reação química descrita por uma propriedade escalar conservada e denominada fração de mistura. O modelo matemático para a fase dispersa, o qual descreve o movimento das gotículas é descrito na Seção 2.4. $\mathrm{Na}$ Seção 2.5 descreve-se o acoplamento entre as fases dispersa e contínua. A Seção 2.6 apresenta os três modelos matemáticos utilizados no presente trabalho: o primeiro para um escoamento incompressível (não-reativo), o segundo para um escoamento incompressível (não-reativo) com uma fase dispersa inerte sem evaporação e o terceiro para um escoamento reativo com baixo número de Mach.

\subsection{Equações para um escoamento reativo na fase contínua}

Para modelar um escoamento multicomponente reativo com baixo número de Mach e $N$ espécies químicas, empregam-se as equações da conservação da massa, da conservação da fração mássica de cada espécie química, da quantidade de movimento linear, da conservação da energia e do estado 
termodinâmico. Tais equações são apresentadas nas próximas seções na notação indicial. Isso permite escrever de forma compacta algumas expressões tratadas no presente trabalho. Com isso, o somatório, $\sum_{j=1}^{3} \frac{\partial u_{i}}{\partial x_{j}}$, é denotado por $\frac{\partial u_{i}}{\partial x_{j}}$, onde $i$ é o índice livre e $j$ é o índice repetido.

\subsubsection{Equação da conservação da massa}

A equação da conservação da massa de cada espécie química $l=1, \ldots, N$ é dada por

$$
\frac{\partial \rho_{l}}{\partial t}+\frac{\partial \dot{m}_{l, j}}{\partial x_{j}}=\dot{\omega}_{l}
$$

sendo $\rho_{l}=\rho Y_{l}$ a massa específica da espécie química $l, \rho$ a massa específica da mistura. O termo $Y_{l}$ é a fração mássica da espécie química $l$ dada por

$$
Y_{l}=\frac{m_{l}}{m}
$$

sendo $m_{l}$ a massa da espécie química $l$ e $m$ a massa da mistura (veja Kuo (2005), Poinsot e Veynante (2005), Turns (1996)). A quantidade $\dot{\mathbf{m}}_{l}=\left(\dot{m}_{l, 1}, \dot{m}_{l, 2}, \dot{m}_{l, 3}\right)$ é o fluxo de massa da espécie química $l$ dado por

$$
\dot{\mathbf{m}}_{l}=\rho Y_{l} \mathbf{v}_{l},
$$

onde $\mathbf{v}_{l}=\left(v_{l, 1}, v_{l, 2}, v_{l, 3}\right)$ é a velocidade da espécie química $l$. O lado direito da equação $(2.1)\left(\dot{\omega}_{l}\right)$ é a taxa de produção ou de consumo da espécie química $l$ devido às reações químicas. A soma das $N$ equações descritas em (2.1) resulta na equação da conservação da massa da mistura

$$
\frac{\partial \rho}{\partial t}+\frac{\partial\left(\rho u_{j}\right)}{\partial x_{j}}=0
$$

onde o campo vetorial $\mathbf{u}=\left(u_{1}, u_{2}, u_{3}\right)$ é a velocidade do escoamento. A equação (2.4) é obtida por meio da relação $\sum_{l=1}^{N} \dot{\mathbf{m}}_{l}=\rho \mathbf{u}$, da Lei da Conservação da Massa $\sum_{l=1}^{N} \rho_{l}=\rho, \sum_{l=1}^{N} Y_{l}=1$ e do somatório nulo $\sum_{l=1}^{N} \dot{\omega}_{l}=0$. Isso implica que enquanto uma espécie é produzida outra é consumida, conforme descrevem Kuo (2005), Poinsot e Veynante (2005) e Versteeg e Malalasekera (2007). Para a dedução da equação da conservação da massa veja Melo e Neto (1991), Batchelor (2000) e Chorin e Marsden (1993).

\subsubsection{Equação da quantidade de movimento linear}

A equação da quantidade de movimento linear é a Segunda Lei de Newton dada por

$$
\frac{\partial\left(\rho u_{i}\right)}{\partial t}+\frac{\partial\left(\rho u_{i} u_{j}\right)}{\partial x_{j}}=-\frac{\partial p}{\partial x_{i}}+\frac{\partial \tau_{j i}}{\partial x_{j}}+F_{i}, \quad \text { para } i=1,2,3
$$

onde $p$ é a pressão, $F_{i}$ são as forças que agem sobre o sistema, dentre as quais inclui-se a aceleração da gravidade. O tensor de tensões viscosas $\tau_{j i}$ é proporcional à taxa de deformação local,

$$
\tau_{i j}=\mu\left(\frac{\partial u_{i}}{\partial x_{j}}+\frac{\partial u_{j}}{\partial x_{i}}\right)+\lambda \delta_{i j} \frac{\partial u_{k}}{\partial x_{k}}
$$


onde $\mu$ é a viscosidade dinâmica (relacionada a deformação linear) e a segunda viscosidade $\lambda$ está relacionada à deformação volumétrica, com a hipótese que $\lambda=-\frac{2}{3} \mu$, veja Versteeg e Malalasekera (2007) e Kuo (2005). O delta de Kronecker, $\delta_{i j}$ na expressão (2.6), é definido por

$$
\delta_{i j}= \begin{cases}1, & \text { se } i=j \\ 0, & \text { se } i \neq j\end{cases}
$$

Para detalhes da dedução da equação da quantidade de movimento linear veja Chorin e Marsden (1993), Melo e Neto (1991), Batchelor (2000) e Kuo (2005).

Utilizando-se a equação da conservação da massa (2.4), obtém-se a forma não conservativa da equação (2.5) dada por

$$
\rho \frac{D u_{i}}{D t}=-\frac{\partial p}{\partial x_{i}}+\frac{\partial \tau_{j i}}{\partial x_{j}}, \text { para } i=1,2,3
$$

onde o operador $\frac{D}{D t}$ é a derivada material definida

$$
\frac{D}{D t}=\frac{\partial}{\partial t}+u_{j} \frac{\partial}{\partial x_{j}}
$$

Para o caso turbulento a viscosidade $\mu$, na expressão (2.6), inclui a viscosidade turbulenta $\mu_{t}$, dada por

$$
\mu_{t}=\left(C_{S} \Delta\right)^{2}|\mathcal{S}|,
$$

onde $C_{S}$ é uma constante do modelo, $\Delta$ é o tamanho do filtro e $\mathcal{S}$ é o tensor taxa de deformação filtrada. A viscosidade turbulenta, dada pela expressão (2.10), é obtida por meio do modelo de turbulência da simulação das grandes escalas (LES - Large Eddy Simulation) descrito no Apêndice A.

\subsubsection{Equação da conservação da fração mássica das espécies químicas}

As espécies químicas são representadas por meio da fração mássica $Y_{l}$ definida pela expressão (2.2). A massa específica, a velocidade e o fluxo de massa de cada espécie química $l$ são dados por $\rho_{l}=\rho Y_{l}, \mathbf{v}_{l}$ e $\dot{\mathbf{m}}_{l}=\rho_{l} \mathbf{v}_{l}$. Da equação (2.1) tem-se que

$$
\frac{\partial\left(\rho Y_{l}\right)}{\partial t}+\frac{\partial\left(\rho Y_{l} v_{l, j}\right)}{\partial x_{j}}=\dot{\omega}_{l}, \quad \text { para } 1 \leq l \leq N
$$

A diferença entre a velocidade de cada espécie química $l$ e a velocidade do escoamento é definida como a velocidade de difusão $\mathbf{v}_{l}^{d}=\mathbf{v}_{l}-\mathbf{u}$, ou seja, a velocidade relativa de uma espécie química em relação a velocidade do escoamento, veja Turns (1996) e Kuo (2005). Desta maneira, obtém-se

$$
\frac{\partial\left(\rho Y_{l}\right)}{\partial t}+\frac{\partial}{\partial x_{j}}\left[\rho Y_{l}\left(u_{j}+v_{l, j}^{d}\right)\right]=\dot{\omega}_{l}, \quad \text { para } 1 \leq l \leq N .
$$

Da Segunda Lei de Fick da Difusão, descrita em Kuo (2005) e Turns (1996), tem-se

$$
\rho Y_{l} v_{l, i}^{d}=-\rho D_{l} \frac{\partial Y_{l}}{\partial x_{i}}, \quad \text { para } 1 \leq l \leq N
$$

sendo $D_{l}$ o coeficiente de difusão binária de massa da espécie química $l$. No presente trabalho, 
assume-se que todas as espécies químicas possuem o mesmo coeficiente de difusão, isto é, $D_{l}=D$ para $1 \leq l \leq N$, veja Poinsot e Veynante (2005), Kuo (2005) e Turns (1996). Desta forma, a equação da conservação da fração mássica de cada espécie química (2.11) pode ser reescrita como

$$
\frac{\partial\left(\rho Y_{l}\right)}{\partial t}+\frac{\partial\left(\rho u_{j} Y_{l}\right)}{\partial x_{j}}=\frac{\partial}{\partial x_{j}}\left(\rho D \frac{\partial Y_{l}}{\partial x_{j}}\right)+\dot{\omega}_{l}, \quad \text { para } 1 \leq l \leq N
$$

O termo da reação química $\dot{\omega}_{l}$ que representa a taxa de produção ou de consumo da espécie química $l$, torna o sistema de equações (2.14) rígido, conforme salientam Bell (2005) e Pember et al. (1998). Para exemplificar o significado deste fato, Butcher (2003) apresenta um exemplo de sistema de equações rígidas originário de um processo químico (veja o Apêndice B).

Outra dificuldade no tratamento da equação (2.14) é sua modelagem não trivial na presença de turbulência do termo $\dot{\omega}_{l}$, conforme descrevem Poinsot e Veynante (2005) (veja mais detalhes no Apêndice A). Para contornar tais dificuldades, foi adotado um modelo químico simplificado com a hipótese de reação química rápida e estequiométrica, o qual considera a natureza global da reação química por meio de uma equação de transporte de um escalar conservado denominado fração de mistura. Esse modelo evita os termos de reação $\dot{\omega}_{l}$ e é descrito na Seção 2.3. Desta maneira, a rigidez associada ao sistema de equações diferenciais oridinárias e o modelo de turbulência para o termo $\dot{\omega}_{l}$, podem ser tratadas com maior facilidade. Em contrapartida, este modelo químico não trata as reações químicas intermediárias e consequentemente, as espécies químicas resultantes destas reações.

\subsubsection{Equação da conservação da energia}

A equação da conservação da energia é a Primeira Lei da Termodinâmica, ou seja, a taxa de variação da energia do escoamento é igual ao calor adicionado ao escoamento menos o trabalho realizado pelo escoamento (veja Kuo (2005), Chorin e Marsden (1993), Turns (1996) e Versteeg e Malalasekera (2007)). Ela é dada por

$$
\frac{D e_{t}}{D t}=-\frac{\partial\left(p u_{i}\right)}{\partial x_{i}}-\frac{\partial \dot{q}_{j}}{\partial x_{j}}+\frac{\partial\left(\tau_{j i} u_{j}\right)}{\partial x_{i}}+\rho \sum_{l=1}^{N} Y_{l} f_{l, j} v_{l, j}+\dot{Q}
$$

onde $e_{t}$ é a energia total do sistema, os termos $\rho \sum_{l=1}^{N} Y_{l} f_{l, j} v_{l, j}$ e $\dot{Q}$ são, respectivamente, a soma do trabalho das forças externas sobre todas as espécies químicas e a taxa de transferência de calor por radiação, e $\dot{\mathbf{q}}=\left(\dot{q}_{1}, \dot{q}_{2}, \dot{q}_{3}\right)$ é o fluxo de calor dado por

$$
\dot{q}_{j}=-\kappa \frac{\partial T}{\partial x_{j}}+\rho \sum_{l=1}^{N} h_{l} Y_{l} v_{l, j}^{d}, \text { para } j=1,2,3 .
$$

No presente trabalho, assumir-se-á que não há transferência de calor por radiação e nem o trabalho das forças externas agindo sobre o sistema. Na expressão (2.16), $\kappa$ é a condutividade térmica do meio e $h_{l}$ é a entalpia absoluta da espécie química $l$, esta dada por

$$
h_{l}=h_{s l}+\Delta h_{l}^{*}, \quad \text { com } h_{s l}=\int c_{p l}(T) d T,
$$


sendo $h_{s l}$ a entalpia sensivel da espécie química $l, \Delta h_{l}^{*}$ a entalpia de formação da espécie química $l$ sob a temperatura de referência $T^{*}$ e $c_{p}$ o calor específico à pressão constante. A entalpia absoluta da mistura é dada por

$$
h=\sum_{l=1}^{N} h_{l} Y_{l} .
$$

A entalpia sensível $h_{s}$ da mistura, é definida de maneira análoga à expressão (2.18). A entalpia total absoluta é a soma da entalpia absoluta da mistura com a energia cinética, dada por

$$
h_{t}=h+\frac{1}{2} \sum_{i=1}^{3} u_{i}^{2}
$$

A energia total do sistema é dada por

$$
e_{t}=h_{t}-\frac{p}{\rho}
$$

A expressão (2.20) também determina a energia sensivel por meio da entalpia sensível e a energia absoluta por meio da entalpia absoluta. Da expressão (2.20) e utilizando a equação da conservação da massa (2.4) obtém-se

$$
\rho \frac{D e_{t}}{D t}=\rho \frac{D h_{t}}{D t}-\frac{D p}{D t}-p \frac{\partial u_{i}}{\partial x_{i}}
$$

A partir das expressões (2.19) e (2.21) obtém-se a equação da energia em termos da entalpia absoluta, da energia cinética, da energia absoluta e da temperatura. O Apêndice C apresenta a dedução das equações (2.22) e (2.23) a partir da equação (2.15).

Assim sendo, as equações da energia em termos da entalpia absoluta e da temperatura conforme descrito em Versteeg e Malalasekera (2007), Poinsot e Veynante (2005), Turns (1996) e Kuo (2005) são dadas por

$$
\begin{aligned}
\rho \frac{D h}{D t} & =\frac{D p}{D t}+\frac{\partial}{\partial x_{j}}\left(\rho D \frac{\partial h}{\partial x_{j}}\right)+\tau_{j i} \frac{\partial u_{j}}{\partial x_{i}}, \\
\rho c_{p} \frac{D T}{D t} & =\frac{D p}{D t}+\frac{\partial}{\partial x_{j}}\left(\kappa \frac{\partial T}{\partial x_{j}}\right)+\tau_{j i} \frac{\partial u_{j}}{\partial x_{i}}+\dot{\omega}_{T},
\end{aligned}
$$

sendo $\dot{\omega}_{T}$ o calor liberado devido às reações químicas, dado por

$$
\dot{\omega}_{T}=-\sum_{l=1}^{N} \Delta h_{l}^{*} \dot{\omega}_{l} .
$$

No presente trabalho, o termo $\tau_{j j} \frac{\partial u_{j}}{\partial x_{i}}$ não será considerado pois assumir-se-á que não há dissipação de energia devido às tensões viscosas. Portanto, as equações (2.22) e (2.23) tornam-se

$$
\begin{gathered}
\frac{\partial(\rho h)}{\partial t}+\frac{\partial\left(\rho u_{j} h\right)}{\partial x_{j}}=\frac{D p}{D t}+\frac{\partial}{\partial x_{j}}\left(\rho D \frac{\partial h}{\partial x_{j}}\right) \\
c_{p}\left[\frac{\partial(\rho T)}{\partial t}+\frac{\partial\left(\rho u_{j} T\right)}{\partial x_{j}}\right]=\frac{D p}{D t}+\frac{\partial}{\partial x_{j}}\left(\kappa \frac{\partial T}{\partial x_{j}}\right)+\dot{\omega}_{T} .
\end{gathered}
$$




\subsubsection{Equação do estado termodinâmico}

Uma equação do estado fornece uma relação entre a pressão $p$, a temperatura $T$ e a massa específica $\rho$ da mistura. Para gases ideais, onde são negligenciados as forças intermoleculares e o volume das moléculas, a equação do estado termodinâmico é dada por

$$
p=\rho R_{M} T
$$

com $R_{M}$ definida por

$$
R_{M}=\frac{\mathcal{R}}{W}=\mathcal{R} \sum_{l=1}^{N} \frac{Y_{l}}{W_{l}},
$$

sendo $W$ o peso molecular da mistura, $\mathcal{R}$ a constante universal dos gases, $Y_{l}$ a fração mássica e $W_{l}$ o peso molecular de cada espécie química $l$.

Os produtos do processo de combustão, conforme Poinsot e Veynante (2005), Turns (1996) e Kuo (2005), podem ser tratados como gases ideais já que as altas temperaturas associadas ao processo de combustão resultam em massas específicas suficientemente baixas.

Assim completa-se o modelo matemático, onde tem-se como incógnitas a massa específica $\rho$ (equação (2.4)), as três componentes da velocidade $\mathbf{u}$ (equação (2.5)), as $N$ espécies químicas $Y$ (equação (2.14)), a temperatura (equação (2.23)) e a pressão (equação (2.27)). A próxima seção apresenta as equações adimensionalizadas para que se possa deduzir o modelo matemático com a hipótese de baixo número de Mach. Além disso, é descrito o modelo de reação química baseada no escalar conservado (denominado fração de mistura) que permite acoplar as equações das espécies químicas e da energia (escrita em termos da temperatura) em uma única equação.

\subsubsection{Equações adimensionalizadas}

Segundo Hou e Mahesh (2005), as equações adimensionais da conservação da massa (2.4), das espécies químicas (2.14), da quantidade de movimento (2.8), da temperatura (2.23) e do estado termodinâmico (2.27) são dadas por

$$
\begin{aligned}
\frac{\partial \rho^{a}}{\partial t^{a}}+\frac{\partial\left(\rho^{a} u_{j}^{a}\right)}{\partial x_{j}^{a}} & =0 \\
\frac{\partial\left(\rho^{a} Y_{l}^{a}\right)}{\partial t^{a}}+\frac{\partial\left(\rho^{a} u_{j}^{a} Y_{l}^{a}\right)}{\partial x_{j}^{a}} & =\frac{1}{R e} \frac{1}{S c} \frac{\partial}{\partial x_{j}^{a}}\left(\rho^{a} D^{a} \frac{\partial Y_{l}^{a}}{\partial x_{j}^{a}}\right)+\dot{\omega}_{l}^{a} \\
\rho^{a} \frac{D u_{i}^{a}}{D t^{a}} & =-\frac{\partial p^{a}}{\partial x_{i}^{a}}+\frac{1}{R e} \frac{\partial \tau_{j i}^{a}}{\partial x_{j}^{a}} \\
\rho^{a} c_{p}^{a} \frac{D T^{a}}{D t^{a}} & =\frac{1}{\operatorname{Pr}} \frac{1}{\operatorname{Re}} \frac{\partial}{\partial x_{j}^{a}}\left(\kappa^{a} \frac{\partial T^{a}}{\partial x_{j}^{a}}\right)+(\gamma-1)(M a)^{2} \frac{D p^{a}}{D t^{a}}+\dot{\omega}_{T}^{a} \\
\frac{\rho^{a} T^{a}}{W^{a}} & =1+\gamma(M a)^{2} p^{a} .
\end{aligned}
$$

Nas equações (2.29)-(2.33), o caracter ${ }^{a}$ indica uma quantidade adimensional. Os números 
adimensionais que caracterizam o escoamento são o número de Mach $(M a)$, o número de Reynolds $(R e)$, o número de Schmidt $(S c)$, e o número de Prandtl $(P r)$ respectivamente dados por

$$
M a=\frac{u^{*}}{c_{s}}=\frac{u^{*}}{\sqrt{\gamma \frac{p^{*}}{\rho^{*}}}}, \quad \operatorname{Re}=\frac{\rho^{*} L u^{*}}{\mu^{*}}, \quad S c=\frac{\mu^{*}}{\rho^{*} D^{*}}, \quad \operatorname{Pr}=\frac{\mu^{*} c_{p}^{*}}{\kappa^{*}},
$$

sendo $\kappa$ a condutividade térmica do escoamento, $D$ o coeficiente de difusão de massa, $c_{s}$ a velocidade do som. O valor $L$ é o comprimento característico do escoamento, $\gamma$ é a razão entre $c_{p}$ (a capacidade de calor à pressão constante) e $c_{v}$ (a capacidade de calor ao volume constante). O caracter * indica os valores característicos do escoamento. A Tabela 2.1 apresenta as unidades de medidas usualmente utilizadas em cada uma das propriedades do escoamento.

Tabela 2.1: Propriedades de referência do escoamento e suas unidades (SI).

\begin{tabular}{|c|c||c|c||c|c|}
\hline Propriedade & Unidade & Propriedade & Unidade & Propriedade & Unidade \\
\hline$\rho^{*}$ & $k g / m^{3}$ & $u^{*}$ & $m / s$ & $L$ & $m$ \\
$D^{*}$ & $m^{2} / s$ & $\mu^{*}$ & $N \times s / m^{2}$ & $c_{p}^{*}$ & $J / k g K$ \\
$\kappa^{*}$ & $J / s \times m \times K$ & $T^{*}$ & $K$ & $\dot{\omega}_{l}^{*}$ & $\mathrm{~kg} / \mathrm{s} \times \mathrm{m}^{3}$ \\
$p^{*}$ & $N / m^{2}$ & $Y_{l}$ & - & & \\
\hline
\end{tabular}

As equações (2.29)-(2.33) são obtidas pela substitução das propriedades adimensionais dadas por

$$
\rho^{a}=\frac{\rho}{\rho^{*}}, u_{i}^{a}=\frac{u_{i}}{u^{*}}, x_{i}^{a}=\frac{x_{i}}{L}, t^{a}=u^{*} \frac{t}{L}, \mu^{a}=\frac{\mu}{\mu^{*}}, T^{a}=\frac{T}{T^{*}}, p^{a}=\frac{p-p^{*}}{\rho^{*} u^{*} u^{*}}, D^{a}=\frac{D}{D^{*}},
$$

nas equações de conservação (2.4), (2.8), (2.14), (2.23) e (2.27). Note que a escolha da pressão adimensionalizada altera as equações adimensionais para a equação da quantidade de movimento linear e da temperatura. Ao escolher $p^{a}=p / p^{*}$, obtêm-se

$$
\begin{aligned}
\rho^{a} \frac{D u_{i}^{a}}{D t^{a}} & =-\frac{1}{\gamma(M a)^{2}} \frac{\partial p^{a}}{\partial x_{i}^{a}}+\frac{1}{R e} \frac{\partial \tau_{j i}^{a}}{\partial x_{j}^{a}}, \quad \text { e } \\
\rho^{a} c_{p}^{a} \frac{D T^{a}}{D t^{a}} & =\frac{1}{\operatorname{Pr}} \frac{1}{R e} \frac{\partial}{\partial x_{j}^{a}}\left(\kappa^{a} \frac{\partial T^{a}}{\partial x_{j}^{a}}\right)+\frac{\gamma-1}{\gamma} \frac{D p^{a}}{D t}+\dot{\omega}_{T}^{a} .
\end{aligned}
$$

Assim, obtém-se as equações adimensionalizadas do modelo matemático. No Apêndice D, encontram-se os detalhes destas deduções.

\subsection{Escoamentos reativos com baixo número de Mach}

Os escoamentos reativos podem ser modelados por meio da formulações incompressível, baixo número de Mach e completamente compressível (veja Poinsot e Veynante (2005)). Na primeira formulação (escoamento incompressível) a massa específica $(\rho)$ é constante. Esta formulação é limitada pela ausência de modificações do escoamento devido a liberação de calor. Na formulação baixo número de Mach a massa específica varia em função da temperatura, mas permanece cons- 
tante em relação ao campo de pressão. As ondas acústicas são eliminadas de forma que o tamanho do passo de integração temporal não seja tão restritivo, veja o Capítulo 3. Esta é uma formulação aceitável para escoamentos subsônicos (baixa velocidade do escoamento em relação à velocidade do som), não sendo possível estudar instabilidades da combustão, onde escoamento, acústica e reações químicas interagem.

No presente trabalho, serão considerados escoamentos reativos com baixo número de Mach, nos quais a massa específica pode mudar devido às variações da temperatura, mas independe da pressão. A formulação para escoamentos com baixo número de Mach adotada explora a separação das escalas de tempo em duas: a do movimento do escoamento e a das ondas acústicas. Essa formulação foi deduzida a partir da análise das expansões assintóticas das equações de NavierStokes, em relação ao número Mach feita por Majda e Sethian (1985) e Rehm e Baum (1978). A ideia é supor que as variáveis são funções do número de Mach. Assim sendo, tomam-se massa específica, campo de velocidade, temperatura e pressão dadas, respectivamente, por

$$
\begin{aligned}
\rho & =\rho_{0}+\rho_{1} \sqrt{\gamma}(M a)+\rho_{2} \gamma(M a)^{2}+O\left((M a)^{3}\right), \\
\mathbf{u} & =\mathbf{u}_{0}+\mathbf{u}_{1} \sqrt{\gamma}(M a)+\mathbf{u}_{2} \gamma(M a)^{2}+O\left((M a)^{3}\right), \\
T & =T_{0}+T_{1} \sqrt{\gamma} M a+T_{2} \gamma(M a)^{2}+O\left((M a)^{3}\right), \\
p & =p_{0}+p_{2} \gamma(M a)^{2}+O\left((M a)^{3}\right),
\end{aligned}
$$

e substitui-se nas equações adimensionalizadas (2.36) e (2.37). Na expansão (2.41), a pressão é escrita em função do número de Mach com base na equação do estado termodinâmico adimensional (2.33). Desta forma, obtém-se para a equação da quantidade de movimento linear

$$
\begin{aligned}
& {\left[\rho_{0}\left(\frac{\partial u_{i, 0}}{\partial t}+u_{j, 0} \frac{\partial u_{i, 0}}{\partial x_{j}}\right)+\frac{1}{\gamma(M a)^{2}} \frac{\partial p_{0}}{\partial x_{i}}-\frac{1}{R e} \frac{\partial \tau_{j i, 0}}{\partial x_{j}}\right]+} \\
& {\left[\rho_{1}\left(\frac{\partial u_{i, 1}}{\partial t}+u_{j, 1} \frac{\partial u_{i, 1}}{\partial x_{j}}\right)-\frac{1}{R e} \frac{\partial \tau_{j i, 1}}{\partial x_{j}}\right] \sqrt{\gamma} M a} \\
& {\left[\rho_{2}\left(\frac{\partial u_{i, 2}}{\partial t}+u_{j, 2} \frac{\partial u_{i, 2}}{\partial x_{j}}\right)+\frac{1}{\gamma(M a)^{2}} \frac{\partial p_{2}}{\partial x_{i}}-\frac{1}{R e} \frac{\partial \tau_{j i, 2}}{\partial x_{j}}\right] \gamma(M a)^{2}=0,}
\end{aligned}
$$

onde $\tau_{j i, r}$ é dado por

$$
\tau_{j i, r}=\mu\left(\frac{\partial u_{i, r}}{\partial x_{j}}+\frac{\partial u_{j, r}}{\partial x_{i}}-\frac{2}{3} \delta_{i j} \frac{\partial u_{k, r}}{\partial x_{k}}\right), \text { para } 0 \leq r \leq 2 .
$$

Para a equação da conservação da energia em termos da temperatura tem-se

$$
\begin{aligned}
& {\left[\rho_{0} c_{p}\left(\frac{\partial T_{0}}{\partial t}+u_{j, 0} \frac{\partial T_{0}}{\partial x_{j}}\right)-\frac{\gamma-1}{\gamma} \frac{D p_{0}}{D t}+\frac{1}{\operatorname{Pr} R e} \frac{\partial}{\partial x_{j}}\left(\kappa \frac{\partial T_{0}}{\partial x_{j}}\right)-\dot{\omega}_{T}\right]+} \\
& {\left[\rho_{1} c_{p}\left(\frac{\partial T_{1}}{\partial t}+u_{j, 1} \frac{\partial T_{1}}{\partial x_{j}}\right)+\frac{1}{\operatorname{PrRe}} \frac{\partial}{\partial x_{j}}\left(\kappa \frac{\partial T_{1}}{\partial x_{j}}\right)\right] \sqrt{\gamma} M a+} \\
& {\left[\rho_{2} c_{p}\left(\frac{\partial T_{2}}{\partial t}+u_{j, 2} \frac{\partial T_{2}}{\partial x_{j}}\right)-\frac{\gamma-1}{\gamma} \frac{D p_{2}}{D t}+\frac{1}{\operatorname{PrRe}} \frac{\partial}{\partial x_{j}}\left(\kappa \frac{\partial T_{2}}{\partial x_{j}}\right)\right] \gamma(M a)^{2}=0 .}
\end{aligned}
$$


Comparando, na equação (2.42) os termos $M a$ de mesma potência obtêm-se

$$
\begin{gathered}
\rho_{0}\left(\frac{\partial u_{i, 0}}{\partial t}+u_{j, 0} \frac{\partial u_{i, 0}}{\partial x_{j}}\right)+\frac{\partial p_{2}}{\partial x_{i}}-\frac{1}{R e} \frac{\partial \tau_{i j, 0}}{\partial x_{j}}=0, \quad \mathrm{e} \\
\frac{\partial p_{0}}{\partial x_{i}}=0 .
\end{gathered}
$$

Como consequência de (2.46), tem-se que $p_{0}$ independe da posição, $p_{0}(\mathbf{x}, t)=p_{0}(t)$. Assim, a expansão (2.41) torna-se

$$
p(\mathbf{x}, t)=p_{0}(t)+\gamma(M a)^{2} p_{2}(\mathbf{x}, t),
$$

onde $p_{0}(t)$ é a componente termodinâmica da pressão e $p_{2}(\mathbf{x}, t)$ é a componente dinâmica da pressão. Comparando na equação (2.44) os termos $M a$ de mesma potência e desprezando-se os termos da ordem de $M a$ e $(M a)^{2}$, obtêm-se,

$$
\rho_{0} c_{p}\left(\frac{\partial T_{0}}{\partial t}+u_{j, 0} \frac{\partial T_{0}}{\partial x_{j}}\right)-\frac{\gamma-1}{\gamma} \frac{\partial p_{0}}{\partial t}-\frac{1}{\operatorname{PrRe}} \frac{\partial}{\partial x_{j}}\left(\kappa \frac{\partial T_{0}}{\partial x_{i}}\right)-\dot{\omega}_{T}=0 .
$$

Desta maneira, as equações da conservação da massa, da quantidade de movimento, temperatura e estado termodinâmico, modificadas com a hipótese de escoamento com baixo número de Mach são

$$
\begin{aligned}
\frac{\partial \rho}{\partial t}+\frac{\partial\left(\rho u_{j}\right)}{\partial x_{j}} & =0 \\
\rho\left(\frac{\partial u_{i}}{\partial t}+u_{j} \frac{\partial u_{i}}{\partial x_{j}}\right) & =-\frac{\partial p_{2}}{\partial x_{i}}+\frac{\partial}{\partial x_{j}}\left[\mu\left(\frac{\partial u_{i}}{\partial x_{j}}+\frac{\partial u_{j}}{\partial x_{i}}-\frac{2}{3} \delta_{i j} \frac{\partial u_{k}}{\partial x_{k}}\right)\right] \\
\rho c_{p} \frac{\partial T}{\partial t}+u_{j} \frac{\partial T}{\partial x_{j}} & =\frac{\partial}{\partial x_{j}}\left(\kappa \frac{\partial T}{\partial x_{j}}\right)+\frac{\gamma-1}{\gamma} \frac{\partial p_{0}}{\partial t}+\dot{\omega}_{T} \\
p_{0} & =\frac{\rho T \mathcal{R}}{W}
\end{aligned}
$$

Assumindo-se que o escoamento ocorre em um ambiente aberto, a pressão termodinâmica $p_{0}$ é constante e a derivada da pressão em relação ao tempo na equação (2.51) desaparece. Para completar o modelo, a próxima seção apresenta o modelo químico utilizado no presente trabalho.

\subsection{Modelo da reação química}

As chamas de difusão constituem uma classe de problemas reativos onde combustível e oxidante não são misturados a nível molecular antes de entrarem na zona de reação. No presente trabalho, a reação química será considerada infinitamente rápida, estequiométrica e irreversível. Para descrever o processo de combustão com esta hipótese será necessário definir um escalar que representará uma quantidade conservada (veja Turns (1996)). Para tanto, considera-se a reação química que envolve somente as espécies químicas combustível $(C)$, oxidante $(O)$ e produto $(P)$, descrita pela reação (lê-se combustível mais $s$ vezes oxidante produz 1 mais $s$ vezes produto)

$$
C+s O \longrightarrow(1+s) P,
$$


sendo $s$ a razão de massa estequiométrica entre as quantidades de oxidante e combustível dada por

$$
s=\left(\frac{Y_{O}}{Y_{C}}\right)_{e s t},
$$

sendo $Y_{O}$ a fração mássica do oxidante e $Y_{C}$ a fração mássica do combustível. A fração mássica $Y_{l}$ de cada espécie $C, O$ e $P$ é descrita pela equação

$$
\frac{\partial \rho Y_{l}}{\partial t}+\frac{\partial\left(\rho u_{j} Y_{l}\right)}{\partial x_{j}}=\frac{\partial}{\partial x_{j}}\left(\rho D \frac{\partial Y_{l}}{\partial x_{j}}\right)+\dot{\omega}_{l}, \text { para } l=C, O, P .
$$

Como a reação química é estequiométrica, as espécies químicas reagentes, combustível e oxidante, são transformadas completamente e instantaneamente em produtos. Desta forma, a taxa de consumo do oxidante satisfaz

$$
\dot{\omega}_{O}=s \dot{\omega}_{C},
$$

e a taxa de reação para a temperatura, segundo Poinsot e Veynante (2005), é dada por

$$
\dot{\omega}_{T}=-\Delta h_{C}^{*} \dot{\omega}_{C}
$$

onde $\Delta h_{C}^{*}$ é a entalpia de formação da espécie química combustível.

Usando as equações (2.51) e (2.55) e as expressões (2.56) e (2.57), obtém-se

$$
\begin{aligned}
& \frac{\partial \rho Y_{C}}{\partial t}+\frac{\partial\left(\rho u_{j} Y_{C}\right)}{\partial x_{j}}=\frac{\partial}{\partial x_{j}}\left(\rho D \frac{\partial Y_{C}}{\partial x_{j}}\right)+\dot{\omega}_{C}, \\
& \frac{\partial \rho Y_{O}}{\partial t}+\frac{\partial\left(\rho u_{j} Y_{O}\right)}{\partial x_{j}}=\frac{\partial}{\partial x_{j}}\left(\rho D \frac{\partial Y_{O}}{\partial x_{j}}\right)+s \dot{\omega}_{C}, \\
& \frac{\partial \rho T}{\partial t}+\frac{\partial\left(\rho u_{j} T\right)}{\partial x_{j}}=\frac{\partial}{\partial x_{j}}\left(\frac{\kappa}{c_{p}} \frac{\partial T}{\partial x_{j}}\right)-\frac{\Delta h_{C}^{*}}{c_{p}} \dot{\omega}_{C} .
\end{aligned}
$$

A fração mássica do produto da reação $Y_{p}$ é obtido a partir da Lei de Conservação de Massa $\sum_{l} Y_{l}=1$, ou seja,

$$
Y_{P}=1-Y_{O}-Y_{C}
$$

Assumindo que o número de Lewis,

$$
L e=\frac{\kappa}{\rho c_{p} D}=\frac{S c}{P r}
$$

é unitário, isto é, $\rho D=\frac{\kappa}{c_{p}}$, espécies químicas e temperatura difundem da mesma maneira. Combinando-se as equações descritas em (2.58) duas a duas, conforme Poinsot e Veynante (2005), definem-se três quantidades escalares

$$
Z_{1}=s Y_{C}-Y_{O}, \quad Z_{2}=\frac{c_{p} T}{\Delta h_{C}^{*}}+Y_{C}, \quad Z_{3}=\frac{s c_{p} T}{\Delta h_{C}^{*}}+Y_{O},
$$

onde $s$ é a razão estequiométrica entre as quantidades de oxidante e combustível. O escalar $Z_{1}$ é denominado fração de mistura. Não é difícil perceber que os três escalares $Z_{i}$, com $i=1,2,3$, 
satisfazem a equação de advecção-difusão sem termo fonte

$$
\frac{\partial \rho Z_{i}}{\partial t}+\frac{\partial\left(\rho u_{j} Z_{i}\right)}{\partial x_{j}}=\frac{\partial}{\partial x_{j}}\left(\rho D \frac{\partial Z_{i}}{\partial x_{j}}\right), \quad \text { para } i=1,2,3 .
$$

Em uma chama de difusão, combustível e oxidante entram separadamente na zona de reação (chama não pré-misturada, como mostra a Figura 1.1). Desta maneira, os escalares $Z_{i}$ assumem dois valores na entrada da zona de reação: $Z_{i, C}$ na região da entrada do combustível e $Z_{i, O}$ na região da entrada do oxidante. Os escalares $Z_{i}$ normalizados são definidos por

$$
z_{i}=\frac{Z_{i}-Z_{i, O}}{Z_{i, C}-Z_{i, O}} \quad \text { para } i=1,2,3
$$

os quais continuam satisfazendo a equação (2.62) e possuem o mesmo valor na entrada da zona de reação, quando $Z_{i}=Z_{i, C}$ tem-se $z_{i}=1$ (indicando a presença de combustível) e quando $Z_{i}=Z_{i, O}$ tem-se $z_{i}=0$ (indicando a presença de oxidante). Assim, pode-se resolver a equação (2.62) para apenas uma das variáveis $z_{i}$, e esta nova variável escalar $z$ é denominada escalar conservado. A introdução do escalar conservado $z$ permite reduzir o número de variáveis do problema e divide o cálculo das espécies químicas e da temperatura em dois subproblemas:

1. determinar a mistura, onde a equação de advecção-difusão (2.62) é solucionada para se obter a variável $z(\mathbf{x}, t)$; e

2. determinar a estrutura da chama, onde as relações entre as frações mássicas $Y_{l}(z)$, a temperatura $T(z)$ e o escalar conservado $z$ são utilizadas.

No presente trabalho, o segundo subproblema é resolvido por meio da estrutura de chama de Burke-Schumann, descrita por Poinsot e Veynante (2005) e Kuo (2005), na qual assume-se que combustível e oxidante não coexistem. A estrutura de chama é definida da seguinte maneira:

se a mistura é rica, $z \geq z_{\text {est }}$ então

$$
\begin{aligned}
Y_{C}(z) & =Y_{C}^{\star} \frac{z-z_{e s t}}{1-z_{e s t}}, \\
Y_{O}(z) & =0 \\
T(z) & =z T_{C}^{\star}+(1-z) T_{O}^{\star}+\frac{\Delta h_{C}^{*} Y_{C}^{\star}}{c_{p}} z_{e s t} \frac{1-z}{1-z_{e s t}},
\end{aligned}
$$

e se a mistura é pobre, $z<z_{\text {est }}$ então

$$
\begin{aligned}
Y_{C}(z) & =0 \\
Y_{O}(z) & =Y_{O}^{\star}\left(1-\frac{z}{z_{e s t}}\right), \\
T(z) & =z T_{C}^{\star}+(1-z) T_{O}^{\star}+\frac{\Delta h_{C}^{*} Y_{C}^{\star}}{c_{p}} z,
\end{aligned}
$$

onde $Y_{C}^{\star}$ e $Y_{O}^{\star}$ são, respectivamente, a fração mássica de combustível e de oxidante na entrada da zona de reação e $T_{O}^{\star}$ e $T_{C}^{\star}$ são, respectivamente, a temperatura do oxidante e do combustível na entrada da zona de reação. 
A posição da chama ocorre onde as frações mássicas das espécies químicas reagentes $Y_{C}$ e $Y_{O}$ são nulas, isto é, combustível e oxidante tornaram-se produtos da reação. Neste local, $z$ é igual ao seu valor estequiométrico $z_{\text {est }}$ definido por

$$
z_{e s t}=\frac{1}{1+\Phi}, \quad \operatorname{com} \Phi=s \frac{Y_{C}^{\star}}{Y_{O}^{\star}}
$$

onde $\Phi$ é chamada razão de equivalência. Além disso, quando $z=z_{\text {est }}$ na equação (2.64), a chama obtém temperatura máxima dada por

$$
T=z_{e s t} T_{C}^{\star}+\left(1-z_{e s t}\right) T_{O}^{\star}+\frac{\Delta h_{C}^{*} Y_{C}^{\star}}{c_{p}} z_{e s t}=\frac{1}{1+\Phi}\left(T_{C}^{\star}+T_{O}^{\star}+\frac{\Delta h_{C}^{*}}{c_{p}} Y_{C}^{\star}\right) .
$$

As espécies químicas reagentes podem estar acompanhadas por espécies químicas inertes, como o nitrogênio $\left(N_{2}\right)$ que não fazem parte da reação química (veja Versteeg e Malalasekera (2007), Turns (1996), Kuo (2005) e Poinsot e Veynante (2005)). A fração mássica da espécie inerte pode ser obtida a partir da relação

$$
Y_{I}(z)=Y_{I, O}(1-z)+Y_{I, C} z
$$

onde $Y_{I, O}$ é o valor da espécie química inerte na região da entrada do oxidante e $Y_{I, C}$ é o valor da espécie química inerte na região da entrada do combustível. Neste caso, a fração mássica dos produtos da combustão é obtida por meio da expressão

$$
Y_{P}=1-Y_{O}-Y_{C}-Y_{I}
$$

Quando o produto da reação contém duas ou mais espécies, a razão entre a massa de cada componente e a massa total pode ser usada para aproximar a fração mássica dos diferentes componentes do produto da reação. Por exemplo, considere a reação química,

$$
Y_{C}+s Y_{O} \longrightarrow \frac{s}{2} Y_{P 1}+\left(1+\frac{s}{2}\right) Y_{P 2}
$$

As razões entre as massas dos componentes e a massa total são, respectivamente,

$$
r_{P 1}=\frac{s / 2}{1+s} \text { e } r_{P 2}=\frac{1+s / 2}{1+s}
$$

Desta forma, tem-se

$$
Y_{P 1}=r_{P 1} Y_{P} \quad \text { e } Y_{P 2}=r_{P 2} Y_{P}
$$

\subsection{Modelo matemático para a fase dispersa de um escoamento incompressível}

No presente trabalho, a fase dispersa é descrita por gotículas inertes que não evaporam e as equações empregadas que a modelam são baseadas nas equações de seu movimento conforme García (2009), Sirignano (1999), Crowe et al. (1998), Turns (1996), Kuo (2005) e Apte et al. (2009).

Os escoamentos contendo uma fase dispersa são descritos por algumas propriedades, como por exemplo, a massa específica, a temperatura de cada gotícula, a velocidade e parâmetros associados ao acoplamento entre as fases, conforme Crowe et al. (1998). Esses escoamentos podem ser clas- 
sificados em dois grupos: os escoamentos densos e os escoamentos diluídos. Segundo Crowe et al. (1998), em um escoamento denso o movimento de uma gotícula é controlado pelas colisões entre as gotículas, enquanto que em um escoamento diluído o movimento de uma gotícula é controlado pelas forças fluído-dinâmicas (arrasto e sustentação).

O número de Stokes St de um escoamento que contém gotículas determina o quanto a velocidade do escoamento influencia na velocidade das gotículas. O número de Stokes é a razão entre o tempo de reação da gotícula $\tau_{p}$ (o tempo que uma gotícula leva para responder a variações do escoamento) e o tempo característico do escoamento $t^{*}$, dado por

$$
S t=\frac{\tau_{p}}{t^{*}}=\tau_{p} \frac{\mathbf{u}^{*}}{L}
$$

onde $\mathbf{u}^{*}$ e $L$ são, respectivamente, a velocidade e o comprimento característico do escoamento da fase contínua. Se $S t \ll 1$ então o tempo de reação das gotículas às mudanças na velocidade do escoamento é muito menor que o tempo característico do escoamento. Neste caso, as gotículas têm tempo para responder às mudanças na velocidade do escoamento e as velocidades do escoamento e das gotículas serão aproximadamente iguais. Por outro lado, se $S t \gg 1$, as gotículas não têm tempo para responder às mudanças na velocidade do escoamento e as velocidades das gotículas são pouco afetadas pela velocidade do escoamento.

No presente trabalho as seguintes hipóteses são assumidas: as gotículas são inertes e esféricas, a densidade da fase dispersa é muito maior que a densidade da fase contínua, as gotículas são tratadas isoladamente (uma gotícula não afeta as outras), a evaporação e os efeitos de deformação são desconsiderados e as gotículas são representadas por pontos no espaço.

\subsubsection{Equações do movimento das gotículas}

A dinâmica das gotículas é modelada empregando uma abordagem lagrangiana. As posições das gotículas são obtidas resolvendo a equação

$$
\frac{d \mathbf{x}_{p}}{d t}=\mathbf{u}_{p}
$$

onde $\mathbf{x}_{p}=\left(x_{p, 1}, x_{p, 2}, x_{p, 3}\right)$ e $\mathbf{u}_{p}=\left(u_{p, 1}, u_{p, 2}, u_{p, 3}\right)$ são, respectivamente, a posição e a velocidade da gotícula $p$.

A equação que descreve a velocidade de cada gotícula $p$ é obtida a partir da Segunda Lei de Newton,

$$
\frac{d\left(m_{p} \mathbf{u}_{p}\right)}{d t}=\mathbf{F}_{p}
$$

onde $m_{p}$ é a massa da gotícula $p$ e $\mathbf{F}_{\mathbf{p}}$ é a soma de todas as forças agindo sobre a gotícula $p$. No presente trabalho, assumir-se-á que as forças agindo sobre a gotícula são a força de arrasto $\mathbf{F}_{p, a}$, a força de empuxo $\mathbf{F}_{p, e}$ e a força gravitacional $\mathbf{F}_{p, g}$. Desta forma, tem-se que

$$
\mathbf{F}_{p}=\mathbf{F}_{p, g}+\mathbf{F}_{p, e}+\mathbf{F}_{p, a}
$$

Sobre uma esfera, as forças descritas na expressão (2.80) são dadas, respectivamente, por

$$
\mathbf{F}_{p, g}+\mathbf{F}_{p, e}=\frac{\pi d_{p}^{3}}{6} \mathbf{g}\left(\rho_{d}-\rho_{c}\right) \text { e } \mathbf{F}_{p, a}=\frac{\pi d_{p}^{2}}{8} C_{a} \rho_{c}\left|\mathbf{u}_{c, p}-\mathbf{u}_{p}\right|\left(\mathbf{u}_{c, p}-\mathbf{u}_{p}\right),
$$


sendo $\mathbf{g}$ a aceleração da gravidade, $\rho_{d}$ a massa específica da fase dispersa, $d_{p}$ o diâmetro da gotícula $p, \rho_{c}$ a massa específica da fase contínua, $\mathbf{u}_{p}$ a velocidade da gotícula $p, \mathbf{u}_{c, p}$ a velocidade da fase contínua na posição da gotícula $p$ e $C_{a}$ o coeficiente de arrasto que, segundo Bini e Jones (2009), é modelado por

$$
C_{a}= \begin{cases}\frac{24}{R e_{p}}\left(1+\frac{R e_{p}^{\frac{2}{3}}}{6}\right), & 0<R e_{p}<1000 \\ 0.424, & R e_{p} \geq 1000 .\end{cases}
$$

Em (2.82), $R e_{p}$ é o número de Reynolds da gotícula $p$, dado por

$$
R e_{p}=\frac{d_{p} \rho_{c}\left|\mathbf{u}_{c, p}-\mathbf{u}_{p}\right|}{\mu_{c}}
$$

sendo $\mu_{c}$ a viscosidade dinâmica da fase contínua. Substituindo a resultante das forças agindo sobre a gotícula $p$ em (2.79) obtém-se

$$
\frac{d\left(m_{p} \mathbf{u}_{p}\right)}{d t}=\frac{\pi d_{p}^{3}}{6} \mathbf{g}\left(\rho_{d}-\rho_{c}\right)+C_{a} \frac{\pi d_{p}^{2}}{8} \rho_{c}\left|\mathbf{u}_{c, p}-\mathbf{u}_{p}\right|\left(\mathbf{u}_{c, p}-\mathbf{u}_{p}\right) .
$$

O lado esquerdo da equação (2.84) pode ser reescrito como

$$
\frac{d\left(m_{p} \mathbf{u}_{p}\right)}{d t}=m_{p} \frac{d \mathbf{u}_{p}}{d t}+\mathbf{u}_{p} \frac{d m_{p}}{d t} .
$$

O termo $\frac{d m_{p}}{d t}$ desaparece da equação (2.85) pois assumiu-se que as gotículas não evaporam. Substituindo (2.85) na equação (2.84) e dividindo-a pela massa da gotícula

$$
m_{p}=\frac{\pi d_{p}^{3} \rho_{d}}{6}
$$

obtém-se

$$
\frac{d \mathbf{u}_{p}}{d t}=\frac{1}{\tau_{p}}\left(\mathbf{u}_{\mathbf{c}, \mathbf{p}}-\mathbf{u}_{\mathbf{p}}\right)+\left(1-\frac{\rho_{c}}{\rho_{d}}\right) \mathbf{g}
$$

onde $\tau_{p}$ é o tempo de reação da gotícula $p$, dado por

$$
\frac{1}{\tau_{p}}=\frac{3 C_{a} \rho_{c}}{4 d_{p} \rho_{d}}\left|\mathbf{u}_{\mathbf{c}, \mathbf{p}}-\mathbf{u}_{\mathbf{p}}\right|
$$

\subsection{Acoplamento entre as fases}

Um aspecto importante na análise de escoamentos bifásicos é o modo como o acoplamento entre as fases é descrito. Se o escoamento de uma fase afeta o outro sem haver o efeito inverso, então o escoamento é acoplado em um sentido apenas. Se existe efeito mútuo então o acoplamento é nos dois sentidos. No presente trabalho, em um escoamento incompressível contendo gotícula inertes, é considerado o acoplamento da quantidade de movimento nos dois sentidos. O acoplamento no sentido da fase contínua para a fase dispersa é feito por meio dos termos indicados com o índice $(c, p)$, descritos na Seção 2.4.1. O acoplamento no sentido da fase dispersa para a fase contínua é feito por meio do acréscimo de um termo fonte, oriundo das propriedades da fase dispersa, na 
equação da quantidade de movimento linear (2.8). Desta forma, tem-se que

$$
S_{u_{i}}=-\frac{1}{V} \sum_{p=1}^{n_{p}} m_{p} \frac{d u_{p, i}}{d t}
$$

onde $n_{p}$ é o número de gotículas em um volume $V$ e $S_{u_{i}}$, com $i=1,2,3$, são os termos fontes associados às equações da quantidade de movimento linear (2.8).

\subsection{Sumário dos modelos matemáticos}

Esta seção apresenta três modelos matemáticos que serão utilizados no presente trabalho. A Seção 2.6.1 apresenta o modelo para um escoamento incompressível (Nós (2007), Roma et al. (1999), Ceniceros et al. (2010b)). A Seção 2.6.2 apresenta o modelo para um escoamento incompressível (não-reativo) com gotículas inertes que não evaporam (veja a Seção 2.4). A Seção 2.6.3 apresenta o modelo para um escoamento reativo com baixo número de Mach.

\subsubsection{Modelo matemático para um escoamento incompressível (não-reativo)}

O modelo matemático para um escoamento incompressível (não-reativo) usado neste trabalho é dado pelas equações

$$
\begin{aligned}
\frac{\partial u_{j}}{\partial x_{j}} & =0 \\
\rho\left(\frac{\partial u_{i}}{\partial t}+u_{j} \frac{\partial u_{i}}{\partial x_{j}}\right) & =-\frac{\partial p}{\partial x_{i}}+\frac{\partial}{\partial x_{j}}\left[\mu\left(\frac{\partial u_{i}}{\partial x_{j}}+\frac{\partial u_{j}}{\partial x_{i}}\right)\right], \text { para } i=1,2,3,
\end{aligned}
$$

onde $\rho$ é a massa específica (constante), $\mathbf{u}=\left(u_{1}, u_{2}, u_{3}\right)$ é o campo de velocidade do escoamento, $p$ é a pressão e $\mu$ é a viscosidade do escoamento (constante). Para o caso turbulento $\mu$ inclui a viscosidade turbulenta, veja o Apêndice A. As equações (2.90)-(2.91) possuem no total quatro variáveis, a pressão $p$ e as três componentes do campo de velocidade $\mathbf{u}$ para um total de quatro equações.

\subsubsection{Modelo matemático para um escoamento incompressível (não-reativo) com gotículas inertes}

O modelo matemático para um escoamento incompressível (não-reativo) com gotículas inertes, usado neste trabalho é dado pelas equações que descrevem o escoamento incompressível na fase contínua e pelas equações que descrevem o movimento das gotículas. O modelo usado para a fase contínua é dado por

$$
\begin{aligned}
\frac{\partial u_{j}}{\partial x_{j}} & =0 \\
\rho_{c}\left(\frac{\partial u_{i}}{\partial t}+u_{j} \frac{\partial u_{i}}{\partial x_{j}}\right) & =-\frac{\partial p}{\partial x_{i}}+\frac{\partial}{\partial x_{j}}\left[\mu\left(\frac{\partial u_{i}}{\partial x_{j}}+\frac{\partial u_{j}}{\partial x_{i}}\right)\right]+S_{u_{i}}, \quad \text { para } i=1,2,3,
\end{aligned}
$$

onde $\rho_{c}$ é a massa específica (constante) da fase contínua, $\mathbf{u}=\left(u_{1}, u_{2}, u_{3}\right)$ é a velocidade do escoamento, $p$ é a pressão e $\mu$ é a viscosidade do escoamento (constante). Para o caso turbulento $\mu$ 
inclui a viscosidade turbulenta, veja o Apêndice A. O termo fonte $S_{u_{i}}$ representa um dos sentidos do acoplamento entre as fases contínua e dispersa dado pela expressão (2.89). O modelo usado para a fase dispersa é dado por

$$
\begin{aligned}
\frac{d \mathbf{u}_{p}}{d t} & =\frac{1}{\tau_{p}}\left(\mathbf{u}_{\mathbf{c}, \mathbf{p}}-\mathbf{u}_{\mathbf{p}}\right)+\left(1-\frac{\rho_{c}}{\rho_{d}}\right) \mathbf{g}, \\
\frac{d \mathbf{x}_{\mathbf{p}}}{d t} & =\mathbf{u}_{\mathbf{p}}, \quad \text { para } p=1,2, \ldots, N_{p}
\end{aligned}
$$

onde $\mathbf{u}_{\mathbf{p}}=\left(u_{p, 1}, u_{p, 2}, u_{p, 3}\right)$ e $\mathbf{x}_{\mathbf{p}}=\left(x_{p, 1}, x_{p, 2}, x_{p, 3}\right)$ são, respectivamente, a velocidade e a posição da gotícula $p, \rho_{d}$ é a massa específica da fase dispersa, $\mathbf{u}_{\mathbf{c}, \mathbf{p}}=\left(u_{c, p, 1}, u_{c, p, 2}, u_{c, p, 3}\right)$ é a velocidade da fase contínua na posição da gotícula $p, \mathbf{g}$ é a aceleração gravitacional, $\tau_{p}$ é o tempo de reação da gotícula $p$ devido às variações da quantidade de movimento do escoamento dado pela expressão (2.88) e $N_{p}$ o número de gotículas presentes no escoamento. As equações (2.92)-(2.93) possuem no total quatro variáveis, a pressão $p$ e as três componentes do campo de velocidade $\mathbf{u}$ para um total de quatro equações. Já as equações (2.94)-(2.95) possuem no total $6 N_{p}$ variáveis, as posições (três coordenadas) e as velocidades (três componentes) de $N_{p}$ gotículas, para um total de $6 N_{p}$ equações.

\subsubsection{Modelo matemático para um escoamento reativo com baixo número de Mach}

O modelo matemático para um escoamento reativo com baixo número de Mach usado no presente trabalho é dado pelas equações

$$
\begin{aligned}
\frac{\partial \rho}{\partial t}+\frac{\partial\left(\rho u_{j}\right)}{\partial x_{j}} & =0 \\
\rho\left(\frac{\partial u_{i}}{\partial t}+u_{j} \frac{\partial u_{i}}{\partial x_{j}}\right) & =-\frac{\partial p_{2}}{\partial x_{i}}+\frac{\partial}{\partial x_{j}}\left[\mu\left(\frac{\partial u_{i}}{\partial x_{j}}+\frac{\partial u_{j}}{\partial x_{i}}-\frac{2}{3} \delta_{i j} \frac{\partial u_{k}}{\partial x_{k}}\right)\right], \text { para } i=1,2,3, \\
\frac{\partial(\rho z)}{\partial t}+\frac{\partial\left(\rho u_{i} z\right)}{\partial x_{i}} & =\frac{\partial}{\partial x_{i}}\left(\rho D \frac{\partial z}{\partial x_{i}}\right), \\
p_{0} & =\frac{\rho T \mathcal{R}}{W}
\end{aligned}
$$

onde $\rho$ é a massa específica, $\mathbf{u}=\left(u_{1}, u_{2}, u_{3}\right)$ é o campo de velocidade do escoamento, $p_{2}$ é a pressão dinâmica, $z$ é o escalar conservado, $p_{0}$ é a pressão termodinâmica (pressão ambiente constante), $T$ é a temperatura, $\mathcal{R}$ é constante universal dos gases, $W$ é o peso molecular da mistura e $\mu$ é a viscosidade do escoamento (constante). Para o caso turbulento $\mu$ inclui a viscosidade turbulenta, veja o Apêndice A. As equações (2.96)-(2.99) possuem no total seis variáveis, a massa específica $\rho$, a pressão $p_{2}$, as três componentes do campo de velocidade $\mathbf{u}$ e o escalar conservado $z$, para um total de seis equações. A temperatura $T$ e as espécies químicas $Y_{C}, Y_{O}$ e $Y_{P}$ são obtidas por meio das expressões (2.64)-(2.69).

Nos próximos capítulos são apresentadas a metodologia numérica proposta, uma técnica de refinamento adaptativo de malhas para a resolução destes problemas, a verificação numérica e os resultados numéricos obtidos. 


\subsection{Nomenclatura do presente capítulo}

$c_{p} \quad$ Calor específico à pressão constante $(J / K g \times K$ ou $J / K m o l \times K)$

$c_{s} \quad$ Velocidade do som $(\mathrm{m} / \mathrm{s})$

$c_{v} \quad$ Calor específico ao volume constante $(J / K g \times K$ ou $J / K m o l \times K)$

$C \quad$ Espécie química combustível

$C_{a} \quad$ Coeficiente de arrasto

$C_{S} \quad$ Constante de Smagorinsky

$d_{p} \quad$ Diâmetro da gotícula $p(m)$

$D \quad$ Coeficiente de difusão de massa $\left(\mathrm{m}^{2} / \mathrm{s}\right)$

$e_{t} \quad$ Energia total $(J$ ou $J / k g)$

$f \quad$ Forças externas $(N)$

g Aceleração gravitacional $\left(\mathrm{m} / \mathrm{s}^{2}\right)$

$h \quad$ Entalpia absoluta $(J / K)$

$h_{s} \quad$ Entalpia sensível $(J / K)$

$h_{t} \quad$ Entalpia total absoluta $(J / K)$

I Espécie química inerte

$\dot{\mathbf{m}} \quad$ Fluxo de massa $(\mathrm{kg} / \mathrm{s})$

$m \quad$ Massa $(\mathrm{kg})$

$N \quad$ Número de espécies químicas

$n_{p} \quad$ Número de gotículas

$O \quad$ Espécie química oxidante

$p \quad$ Pressão (atm)

$p_{0} \quad$ Pressão termodinâmica $(\mathrm{atm})$

$p_{2} \quad$ Pressão dinâmica $(\mathrm{atm})$

$P \quad$ Espécie química produto

$\dot{\mathbf{q}} \quad$ Fluxo de calor $(\mathrm{W} / \mathrm{kg})$

$\dot{Q} \quad$ Taxa de transferência de calor (por radiação) $(W / k g)$

$r_{p} \quad$ Raio da gotícula $(m)$

$\mathcal{R} \quad$ Constante universal dos gases $(\mathrm{J} / \mathrm{Kmol} \times \mathrm{K})$

$R_{M}$ Parâmetro da equação do estado termodinâmico $(J / k g \times K)$

$s \quad$ Razão estequiométrica entre a fração mássica de oxidante e combustível

$\mathcal{S}$ Tensor taxa de deformação filtrado

$t \quad$ Tempo $(s)$

$T$ Temperatura $(K)$

u Velocidade do escoamento $(\mathrm{m} / \mathrm{s})$

$\mathbf{v}^{d} \quad$ Velocidade de difusão das espécies $(\mathrm{m} / \mathrm{s})$

$V \quad$ Volume $\left(m^{3}\right)$

$W \quad$ Peso molecular $(\mathrm{kg} / \mathrm{Kmol})$

$\mathbf{x}_{p} \quad$ Posição de uma gotícula $(m)$

$Y \quad$ Fração mássica de uma espécie química

$z \quad$ Escalar conservado normalizado

$Z \quad$ Escalar conservado 


\section{Letras Gregas}

$\delta_{i j} \quad$ Delta de Kronecker

$\Delta \quad$ Tamanho do filtro

$\Delta h \quad$ Entalpia de formação $(J / K g$ ou $J / K m o l)$

$\Phi \quad$ Razão de equivalência

$\gamma \quad$ Razão entre $c_{p}$ e $c_{v}$

$\kappa \quad$ Condutividade térmica $(\mathrm{kg} / \mathrm{m} \times \mathrm{s})$

$\mu \quad$ Viscosidade dinâmica $\left(N \times s / m^{2}\right)$

$\mu_{t} \quad$ Viscosidade turbulenta $\left(N \times s / m^{2}\right)$

$\rho \quad$ Massa específica, densidade $\left(\mathrm{kg} / \mathrm{m}^{3}\right)$

$\tau_{j i} \quad$ Tensor de tensões viscosas $\left(N / m^{2}\right)$

$\tau_{p} \quad$ Tempo de reação de uma gotícula $(s)$

$\dot{\omega} \quad$ Taxa de produção/consumo das espécies $\left(\mathrm{kg} / \mathrm{s} \times \mathrm{m}^{3}\right)$

$\dot{\omega}_{T} \quad$ Calor liberado devido às reações químicas $\left(W / m^{3}\right)$

\section{Adimensionais}

Ma Número de Mach Le Número de Lewis

$\operatorname{Pr} \quad$ Número de Prandtl Re Número de Reynolds

$S c \quad$ Número de Schimdt St Número de Stokes

\section{Índices}

$\begin{array}{llll}a & \text { Adimensional } & c & \text { Fase contínua } \\ d & \text { Fase dispersa } & i, j, k & \text { Notação indicial } \\ l & \text { Espécie química } & p & \text { Gotícula } \\ \text { est } & \text { Valores estequiométricos } & * & \text { Valores característicos } \\ \star & \text { Valores na entrada da zona de reação } & C & \text { Espécie química combustível } \\ O & \text { Espécie química oxidante } & P & \text { Espécie química produto }\end{array}$

\section{Operadores}

$\frac{D}{D t}=\frac{\partial}{\partial t}+u_{i} \frac{\partial}{\partial x_{i}} \quad$ Derivada total

Tabela de Conversões

$W-J / s \quad J-k g / m^{2} \times s^{2} \quad J-N m \quad N-P a \times m^{2}$ 


\section{Capítulo 3}

\section{Metodologia numérica}

Neste capítulo são apresentadas as metodologias que serão aplicadas para obter as soluções numéricas dos modelos apresentados no capítulo anterior. As fases do escoamento são aproximadas por um método Euleriano-Lagrangiano. A fase contínua é aproximada na forma euleriana, ou seja, por meio das equações que descrevem um escoamento reativo com baixo número de Mach discretizadas em uma malha Cartesiana bloco-estruturada. A fase dispersa é aproximada na forma lagrangiana, onde as propriedades de cada gotícula (posição, velocidade e diâmetro) são calculadas em termos da sua trajetória. Para a discretização temporal da fase contínua são utilizados esquemas Implicítos-Explícitos, (IMEX), de segunda ordem, descritos por Ascher et al. (1995) e Wang e Ruuth (2008). Para a fase dispersa, é utilizado o método de Euler Modificado que também possui segunda ordem de aproximação. Uma versão da extensão do método da projeção, proposto por Pember et al. (1998), é utilizado para desacoplar a pressão na equação da quantidade do movimento linear. Este método é baseado no método da Projeção de Chorin (1968) e Temam (1968). Para o acoplamento entre as fases contínua e dispersa é utilizada a funçâo Delta de Dirac (veja Griffith (2005) e Roma (1996)). A discretização espacial é feita por meio do esquema de diferenças finitas de segunda ordem (veja Strikwerda (2004)). As soluções dos sistemas lineares originados das discretizações temporal e espacial é aproximada por meio do método Multigrid conforme Roma (1996) e Nós (2007), para mais detalhes veja Briggs e McCormick (2000) e Trottenberg et al. (2001).

O capítulo é dividido em três seções. A Seção 3.1 apresenta o método da projeção de ChorinTeman e uma versão da extensão do método da projeção para escoamento reativos com baixo número de Mach. A Seção 3.2 apresenta a metodologia numérica utilizada para aproximar a fase contínua do escoamento. Esta metodologia inclui: a discretização temporal da fase contínua, a discretização espacial e o método Multigrid para a solução dos sistemas lineares. A Seção 3.3 apresenta a metodologia numérica para a fase dispersa, a qual inclui a discretização temporal e os operadores de interpolação e espalhamento, responsáveis pela troca de informações entre as fases.

\subsection{Método da Projeção}

A solução numérica das equações que modelam um escoamento reativo com baixo número de Mach são calculadas por meio de uma versão estendida de um método da projeção (veja Pember et al. (1998)), o qual é uma variação do método da Projeção de Chorin-Temam para um escoamento incompressível (veja Roma (1996), Griffith (2005), Nós (2007) e Ceniceros et al. 
$(2010 b))$.

O objetivo do método da projeção é desacoplar, na equação da quantidade de movimento linear (2.5), os cálculos do campo de velocidade e da pressão. Em trabalhos independentes, Chorin (1968) e Temam (1968) constataram que para escoamentos incompressíveis a pressão não possui significado termodinâmico, mas força a condição de incompressibilidade.

O método da projeção pode ser resumido em dois passos:

1. Determinar uma aproximação para um campo de velocidade auxiliar $\mathbf{u}^{\star}$ solucionando um problema parabólico.

2. Resolver uma equação elíptica para garantir a condição de incompressibilidade e obter uma correção usada para determinar a pressão e corrigir o campo de velocidade $\mathbf{u}$.

O método da projeção é um método de passo fracionado, diferindo dos demais métodos na maneira de avançar o campo de velocidade auxiliar e corrigir a pressão, conforme apresentam Chorin (1968), Kim e Moin (1985), Roma (1996), Bell et al. (1989) e Griffith (2005). Alguns métodos de passo fracionado resolvem a pressão diretamente na equação elíptica, como descreve Ferziger e Perić (1999).

O método da Projeção de Chorin-Temam para escoamentos incompressíveis é baseado no Teorema da Decomposição de Helmholtz-Hodge descrito pelo Teorema 1.

Teorema 1 Seja $\Omega$ uma região no espaço (ou no plano) com fronteira $\partial \Omega$ suave. Um campo vetorial $\mathbf{w}$ em $\Omega$ pode ser decomposto de maneira única como

$$
\mathbf{w}=\mathbf{u}_{d}+\nabla \phi,
$$

onde $\nabla \cdot \mathbf{u}_{d}=0$, $\phi$ é uma propriedade escalar e $\mathbf{u}_{d}$ satisfaz $\mathbf{u}_{d} \cdot \mathbf{n}=0$ em $\partial \Omega$.

Para a demonstração do Teorema 1 veja Chorin e Marsden (1993).

A decomposição descrita na expressão (3.1) pode ser vista como a projeção do campo vetorial no espaço dos campos vetoriais com divergente nulo. Desta forma, o campo de velocidade auxiliar obtido no passo 1 é projetado no espaço dos campos vetoriais com divergente nulo. A transformação que leva o campo de velocidade auxiliar no espaço dos campos vetoriais com divergente nulo é o operador de projeção $\mathcal{P}$ dado por

$$
\mathcal{P}=\mathcal{I}-\frac{1}{\rho} \nabla\left\{\left[\nabla \cdot\left(\frac{1}{\rho} \nabla\right)\right]^{-1}\right\} \nabla \cdot
$$

Desta maneira, a decomposição do campo de velocidade auxiliar é dada por

$$
\mathbf{u}^{\star}=\mathbf{u}+\frac{1}{\rho} \nabla \phi,
$$

sendo $\phi$ uma correção para o campo de velocidade $\mathbf{u}$ e para a pressão $p$. Para determinar a correção $\phi$, aplica-se o operador $\nabla \cdot$ na equação (3.3) obtendo-se

$$
\nabla \cdot\left(\frac{1}{\rho} \nabla \phi\right)=\nabla \cdot \mathbf{u}^{\star}
$$

pois $\nabla \cdot \mathbf{u}=0$. 


\subsubsection{Extensão do método da projeção para escoamentos reativos com baixo número de Mach}

A extensão do método da projeção para escoamentos reativos com baixo número de Mach (Pember et al. (1998)) é baseada na expansão das equações de Navier-Stokes em relação ao número de Mach proposta por Majda e Sethian (1985) e Rehm e Baum (1978). Segundo Pember et al. (1998) a hipótese do modelo para baixo número de Mach assegura que para um escoamento em um domínio espacial aberto com o número de Mach suficientemente pequeno $(M a<0.3)$, a pressão $p$ pode ser decomposta como uma soma

$$
p(\mathbf{x}, t)=p_{0}+p_{2}(\mathbf{x}, t),
$$

onde $\frac{p_{2}(\mathbf{x}, t)}{p_{0}}=O\left((M a)^{2}\right), p_{0}$ é a pressão termodinâmica constante e $p_{2}$ é a pressão dinâmica. Todas as propriedades termodinâmicas são independentes de $p_{2}$ (veja a Seção 2.2).

Os escoamentos reativos com baixo número de Mach incluem variações de densidade de amplitude finita, e a restrição no divergente do campo de velocidade não é homogênea, como o é para escoamentos incompressíveis. A extensão da metodologia para escoamentos reativos com baixo número de Mach também se baseia no Teorema 1 e mantém o mesmo operador de projeção definido pela expressão (3.2). A diferença entre o método da projeção e a sua extensão para escoamentos reativos com baixo número de Mach está na maneira de decompor o campo de velocidade auxiliar. Define-se $\nabla \cdot \nabla \xi=S$, sendo $S=\nabla \cdot \mathbf{u}$ e decompõe-se o campo de velocidade auxiliar da seguinte maneira

$$
\mathbf{u}^{\star}=\mathbf{u}_{d}+\nabla \xi+\frac{1}{\rho} \nabla \phi,
$$

sendo $\nabla \cdot \mathbf{u}_{d}=0, \nabla \cdot \mathbf{u}^{\star} \neq 0, \phi$ e $\xi$ variáveis escalares. Desta forma, projetando-se o campo vetorial $\mathbf{u}^{\star}-\nabla \xi$ no espaço vetorial dos campos com divergente nulo, pode-se escrever o campo de velocidade como segue

$$
\mathbf{u}=\mathbf{u}_{d}+\nabla \xi
$$

Aplicando-se o operador $\nabla \cdot$ na equação (3.7) verifica-se a condição $\nabla \cdot \mathbf{u}=S$. A expressão para o termo $S$ é obtida por intermédio da equação da conservação da massa,

$$
\frac{\partial \rho}{\partial t}+\frac{\partial\left(u_{j} \rho\right)}{\partial x_{j}}=0
$$

e da equação do estado termodinâmico

$$
p_{0}=\frac{\rho \mathcal{R} T}{W} .
$$

Derivando-se a equação (3.9) em relação ao tempo obtém-se

$$
\frac{D p_{0}}{D t}=\frac{T \mathcal{R}}{W} \frac{D \rho}{D t}+\frac{\rho \mathcal{R}}{W} \frac{D T}{D t}+T \rho \mathcal{R} \sum_{l} \frac{1}{W_{l}} \frac{D Y_{l}}{D t}, \quad \text { para } l=C, O, P .
$$

onde $p_{0}$ é a pressão termodinâmica, $T$ é a temperatura, $W$ é o peso molecular da mistura, $\rho$ é a 
massa específica da mistura, $Y_{l}$ e $W_{l}$, são respectivamente, a fração mássica e o peso molecular da espécie química $l$. Como $p_{0}$ é contante e substituindo-se a equação da conservação da massa,

$$
\frac{D \rho}{D t}=-\rho \nabla \cdot \mathbf{u},
$$

na expressão (3.10) obtém-se

$$
\nabla \cdot \mathbf{u}=\frac{1}{T} \frac{D T}{D t}+W \sum_{l} \frac{1}{W_{l}} \frac{D Y_{l}}{D t}, \text { para } l=C, O, P
$$

Assim, a restrição para o divergente da velocidade para um escoamento reativo com baixo número de Mach é dada pela expressão

$$
\begin{aligned}
S & =\frac{1}{T}\left(\frac{\partial T}{\partial t}+u_{j} \frac{\partial T}{\partial x_{j}}\right)+W \sum_{l}\left[\frac{1}{W_{l}}\left(\frac{\partial Y_{l}}{\partial t}+u_{j} \frac{\partial Y_{l}}{\partial x_{j}}\right)\right] \\
& =\frac{1}{\rho T}\left(\frac{\partial(\rho T)}{\partial t}+\frac{\partial\left(\rho u_{j} T\right)}{\partial x_{j}}\right)+\frac{W}{\rho} \sum_{l}\left[\frac{1}{W_{l}}\left(\frac{\partial\left(\rho Y_{l}\right)}{\partial t}+\frac{\partial\left(\rho u_{j} Y_{l}\right)}{\partial x_{j}}\right)\right],
\end{aligned}
$$

para $l=C, O, P$.

A equação para a correção $\phi$ é obtida ao aplicar o operador $\nabla \cdot$ na expressão (3.6), obtendo-se

$$
\nabla \cdot\left(\frac{1}{\rho} \nabla \phi\right)=\nabla \cdot \mathbf{u}^{\star}-S
$$

pois $\nabla \cdot \mathbf{u}_{d}=0$ e $\nabla \cdot \nabla \xi=S$, note que $\nabla \cdot=\frac{\partial}{\partial x_{j}}$.

\subsection{Solução das equações que modelam a fase contínua}

\subsubsection{Discretização temporal}

Considere a equação

$$
\frac{\partial u(t)}{\partial t}=g(u(t))+f(u(t)),
$$

onde $g(u(t))$ representa o termo advectivo e $f(u(t))$ o termo difusivo. Wang e Ruuth (2008) e Ascher et al. (1995) propõe esquemas temporais denominados IMEX (métodos Implícitos-Explícitos) pois tratam explicitamente o termo advectivo e implicitamente o termo difusivo. O tratamento explícito do termo advectivo evita a resolução de um sistema não linear e o tratamento implícito do termo difusivo evita a escolha de um tamanho de passo $\Delta t$ muito restritivo (da ordem de $(\Delta x)^{2}$, onde $\Delta x=\min \left\{\Delta x_{1}, \Delta x_{2}, \Delta x_{3}\right\}$ e $\Delta x_{i}$ é o espaçamento na direção $x_{i}$, com $\left.i=1,2,3\right)$.

Os métodos IMEX são obtidos por meio de uma expansão em série de Taylor da equação (3.15). No trabalho de Wang e Ruuth (2008) é apresentada uma forma geral de um método de $s$ passos, para a qual os autores mostram que com um tamanho de passo $\Delta t$ variável, o método possui ordem de aproximação $s$. No presente trabalho, serão apresentados os esquemas IMEX de dois passos. Considere três instantes de tempo $t^{n-1}, t^{n}$ e $t^{n+1}$, de forma que o tamanho do passo seja $\Delta t^{n}=t^{n}-t^{n-1}$ e $\Delta t^{n+1}=t^{n+1}-t^{n}$. A forma geral dos esquemas de dois passos com um tamanho 
de passo de integração variável é dada pela expressão

$$
\frac{\alpha_{2} u^{n+1}+\alpha_{1} u^{n}+\alpha_{0} u^{n-1}}{\Delta t^{n+1}}=\theta_{2} f(u)^{n+1}+\theta_{1} f(u)^{n}+\theta_{0} f(u)^{n-1}+\beta_{1} g(u)^{n}+\beta_{0} g(u)^{n-1},
$$

onde $u^{n}$ é uma aproximação para $u\left(t^{n}\right)$ e $\alpha_{i}, \beta_{i}$ e $\theta_{i}$ são parâmetros que dependem de $\Delta t^{n+1}$ e $\Delta t^{n}$, com $\alpha_{2} \neq 0$ e $\theta_{2} \neq 0$. A apresentação paramétrica dos esquemas IMEX em função de $\alpha_{i}, \beta_{i}$ e $\theta_{i}$ facilita a escolha de diferentes métodos. Para os esquemas de dois passos é possível escolher entre os métodos Cranck-Nicolson Adams-Bashforth (CNAB), Cranck-Nicolson Adams-Bashforth Modificado (MCNAB), Gear extrapolado e Cranck-Nicolson Leap-Frog (CNLF). Os parâmetros $\alpha_{i}, \beta_{i}$ e $\theta_{i}$ são dados, respectivamente, por

$$
\begin{gathered}
\alpha_{2}=\frac{\Delta t^{n}+2 \gamma \Delta t^{n+1}}{\Delta t^{n}+\Delta t^{n+1}}, \alpha_{1}=\frac{\Delta t^{n+1}-\Delta t^{n}-2 \gamma \Delta t^{n+1}}{\Delta t^{n}}, \alpha_{0}=-\alpha_{2}-\alpha_{1}, \\
\beta_{1}=\frac{\Delta t^{n}+\gamma \Delta t^{n+1}}{\Delta t^{n}}, \beta_{0}=-\frac{\gamma \Delta t^{n+1}}{\Delta t^{n}}, \\
\theta_{2}=\gamma+c \frac{\Delta t^{n+1}}{\Delta t^{n}+\Delta t^{n+1}}, \theta_{1}=1-\gamma-c \frac{\Delta t^{n+1}}{\Delta t^{n}}, \quad \text { e } \theta_{0}=c\left(\frac{\Delta t^{n+1}}{\Delta t^{n}}-\frac{\Delta t^{n+1}}{\Delta t^{n}+\Delta t^{n+1}}\right),
\end{gathered}
$$

onde $c$ e $\gamma$ são parâmetros que definem o método escolhido. Nas expressões (3.17)-(3.19), os esquemas CNAB, MCNAB, Gear e CNLF são obtidos com as seguintes escolhas para o par de parâmetros $(c, \gamma)$, respectivamente, $\left(0, \frac{1}{2}\right),\left(\frac{1}{8}, \frac{1}{2}\right),(0,1)$ e $(1,0)$. Os equemas temporais IMEX de dois passos precisam de um esquema temporal de um passo para aproximar o primeiro passo de integração. A forma geral dos esquemas IMEX de um passo é dada pela expressão,

$$
\frac{u^{n+1}-u^{n}}{\Delta t^{n+1}}=(1-\theta) f(u)^{n}+\theta f(u)^{n+1}+g(u)^{n} .
$$

Na expressão (3.20), é possível escolher entre os métodos Cranck-Nicolson com $\theta=\frac{1}{2}$ e Euler semi-implícito $\operatorname{com} \theta=1$.

\subsubsection{Estratégia semi-implícita}

Na equação da quantidade de movimento linear (2.5), Nós (2007) utilizou a estratégia semiimplícita proposta por Gottlieb e Orszag (1993) e Douglas e Dupont (1971) para tratar a não linearidade do termo difusivo. A ideia funciona bem para equações predominantemente difusivas, porém é menos eficiente para problemas dispersivos, conforme descreve Nós (2007).

A ideia é reescrever a equação da quantidade de movimento como

$$
\frac{\partial u_{i}}{\partial t}=\frac{\lambda}{\rho} \frac{\partial^{2} u_{i}}{\partial x_{j}^{2}}-\frac{1}{\rho} \frac{\partial p_{2}}{\partial x_{i}}+\tilde{g}_{i}, \text { para } i=1,2,3,
$$

sendo $\lambda=c_{1}|\mu|_{\max }$, com $c_{1}>0$ (para mais detalhes veja Nós (2007), Gottlieb e Orszag (1993) e Douglas e Dupont (1971)), $\rho$ a massa específica da mistura, $\mathbf{u}=\left(u_{1}, u_{2}, u_{3}\right)$ o campo de velocidade do escoamento e $p_{2}$ a pressão dinâmica. O termo $\tilde{g}_{i}$ é dado por

$$
\tilde{g}_{i}=\frac{1}{\rho}\left\{\frac{\partial}{\partial x_{j}}\left[\mu\left(\frac{\partial u_{j}}{\partial x_{i}}+\frac{\partial u_{i}}{\partial x_{j}}-\frac{2}{3} \delta_{i j} \frac{\partial u_{k}}{\partial x_{k}}\right)\right]-\lambda \frac{\partial^{2} u_{i}}{\partial x_{j}^{2}}+F_{i}\right\}-\rho\left(u_{j} \frac{\partial u_{i}}{\partial x_{j}}\right),
$$


sendo $\mu$ a viscosidade dinâmica mais a viscosidade turbulenta e $F_{i}$ o termo que contém as forças que agem sobre o sistema.

Assim a aproximação temporal para as equações (3.21) são dadas por

$$
\frac{\alpha_{2} u_{i}^{n+1}+\alpha_{1} u_{i}^{n}+\alpha_{0} u_{i}^{n-1}}{\Delta t^{n+1}}=\theta_{2} \tilde{f}_{i}^{n+1}+\theta_{1} \tilde{f}_{i}^{n}+\theta_{0} \tilde{f}_{i}^{n-1}+\beta_{1} \tilde{g}_{i}^{n}+\beta_{0} \tilde{g}_{i}^{n-1}, \quad \text { para } i=1,2,3,
$$

sendo $\tilde{g}_{i}$ dado pela expressão $(3.22)$ e $\tilde{f}_{i}$ dado por

$$
\tilde{f}_{i}=\frac{\lambda}{\rho} \frac{\partial^{2} u_{i}}{\partial x_{j}^{2}}-\frac{1}{\rho} \frac{\partial p_{2}}{\partial x_{i}}
$$

Para a equação do transporte do escalar conservado (2.62), também foi utilizada a estratégia semi-implícita descrita para a equação da quantidade de movimento. Assim a aproximação temporal para a equação do escalar conservado (fração de mistura) via método IMEX é dada por

$$
\begin{aligned}
\frac{\alpha_{2}(\rho z)^{n+1}+\alpha_{1}(\rho z)^{n}+\alpha_{0}(\rho z)^{n-1}}{\Delta t^{n+1}} & =\theta_{2}\left(\tilde{D} \frac{\partial^{2} z}{\partial x_{j}^{2}}\right)^{n+1}+\theta_{1}\left(\tilde{D} \frac{\partial^{2} z}{\partial x_{j}^{2}}\right)^{n}+\theta_{0}\left(\tilde{D} \frac{\partial^{2} z}{\partial x_{j}^{2}}\right)^{n-1}+ \\
& +\beta_{1} \tilde{g}_{0}^{n}+\beta_{0} \tilde{g}_{0}^{n-1}
\end{aligned}
$$

sendo $\tilde{g}_{0}$ e $\tilde{D}$, respectivamente,

$$
\tilde{g}_{0}=\frac{\partial}{\partial x_{j}}\left(\rho D \frac{\partial z}{\partial x_{j}}\right)-\frac{\partial \rho u_{j} z}{\partial x_{j}}-\tilde{D} \frac{\partial^{2} z}{\partial x_{j}^{2}}, \quad \text { e } \widetilde{D}=c_{1}|\rho D|_{\max }, \quad \text { com } c_{1}>0 .
$$

A condição de estabilidade dos esquemas numéricos IMEX para a fase contínua é dada pela condição de estabilidade CFL, descrita por Strikwerda (2004), dada por

$$
\Delta t_{c}=C \min \left(\frac{\Delta x_{1}}{\left|u_{1}\right|_{\max }}, \frac{\Delta x_{2}}{\left|u_{2}\right|_{\max }}, \frac{\Delta x_{3}}{\left|u_{3}\right|_{\max }}\right), \quad \text { com } 0<C<1,
$$

já que os termos difusivos nas equações (3.23) e (3.25) são tratados implicitamente.

\subsubsection{Discretização do domínio computacional}

As equações discretas para a fase contínua definidas em um domínio físico $\Omega=\left[a_{1}, b_{1}\right] \times\left[a_{2}, b_{2}\right] \times$ $\left[a_{3}, b_{3}\right]$, são aproximadas em uma malha computacional bloco-estruturada refinada localmente que cobre $\Omega$. Nesta seção são apresentados os elementos de uma malha Cartesiana estruturada uniforme. No Capítulo 4 são descritas as propriedades de uma malha bloco-estruturada com refinamento adaptativo.

Uma célula computacional do domínio $\Omega$ corresponde a um paralelepípedo de dimensões $\Delta x_{1}$, $\Delta x_{2}$ e $\Delta x_{3}$, com

$$
\Delta x_{1}=\frac{b_{1}-a_{1}}{n_{1}}, \quad \Delta x_{2}=\frac{b_{2}-a_{2}}{n_{2}}, \quad \Delta x_{3}=\frac{b_{3}-a_{3}}{n_{3}},
$$

e $n_{i}$, com $i=1,2,3$ é o número de partições em cada uma direções. A posição do centro de cada 
célula computacional é dada por

$$
\mathbf{x}_{i j k}=\left(x_{1, i}, x_{2, j}, x_{3, k}\right)=\left(a_{1}+\left(i-\frac{1}{2}\right) \Delta x_{1}, a_{2}+\left(j-\frac{1}{2}\right) \Delta x_{2}, a_{3}+\left(k-\frac{1}{2}\right) \Delta x_{3}\right),
$$

para $1 \leq i \leq n_{1}, 1 \leq j \leq n_{2}$ e $1 \leq k \leq n_{3}$.

Dado que o domínio computacional está coberto por células computacionais, é necessário definir em qual posição da célula serão aproximados os valores de cada variável tratada. No presente trabalho é utilizado o modelo MAC (marker and cell): a aproximação de grandezas vetoriais é feita nas faces de cada célula computacional da malha Cartesiana e a aproximação das variáveis escalares é feita no centro de cada célula computacional, como mostra a Figura 3.1.

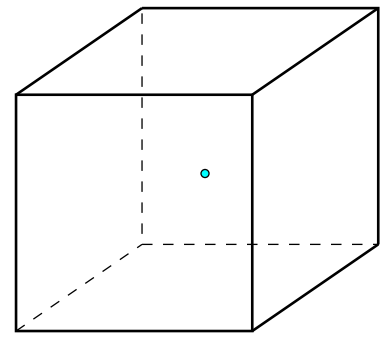

(a)

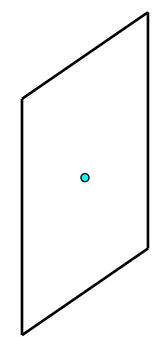

(b)

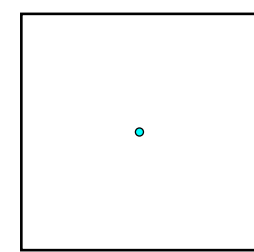

(c)

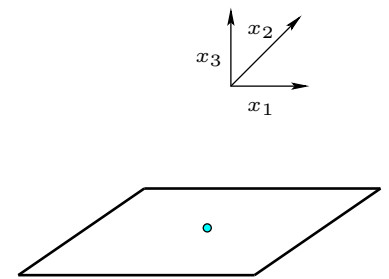

(d)

Figura 3.1: Localização das variáveis escalares e vetoriais discretizadas em uma célula computacional. (a) Variáveis escalares no centro de uma célula computacional. (b) Componente da velocidade na direção $x_{1}$ no centro da face $x_{2} x_{3}$. (c) Componente da velocidade na direção $x_{2}$ no centro da face $x_{1} x_{3}$. (d) Componente da velocidade na direção $x_{3}$ no centro da face $x_{1} x_{2}$.

As variáveis escalares localizadas no centro de cada célula computacional são denotadas por $\phi_{i j k}$, enquanto que as componentes do campo de velocidade $\mathbf{u}=\left(u_{1}, u_{2}, u_{3}\right)$, localizadas nas faces de cada célula computacional são denotadas por

$$
\mathbf{u}_{i j k}=\left(u_{1, i-\frac{1}{2} j k}, u_{2, i j-\frac{1}{2} k}, u_{3, i j k-\frac{1}{2}}\right) \text {. }
$$

\subsubsection{Discretização espacial}

As equações que descrevem a fase contínua são aproximadas por diferenças finitas de segunda ordem, em uma malha Cartesiana deslocada, adaptativa e bloco-estruturada. A malha Cartesiana refinada localmente e a estratégia do refinamento adaptativo serão descritas no Capítulo 4 . Na malha Cartesiana deslocada, veja Fortuna (2000) e Trottenberg et al. (2001), são introduzidos os operadores de interpolação e de diferenças finitas para as aproximações dos operadores diferenciais espaciais.

Desta forma, os operadores diferenciais discretos: gradiente $(\mathbf{G})$, divergente $(\mathbf{D})$ e laplaciano (L) são definidos, respectivamente, pelas expressões

$$
\begin{aligned}
(\mathbf{G} \phi)_{i j k} & =\left(\left(\mathbf{D}_{\mathbf{1}} \phi\right)_{i-\frac{1}{2} j k},\left(\mathbf{D}_{\mathbf{2}} \phi\right)_{i j-\frac{1}{2} k},\left(\mathbf{D}_{\mathbf{3}} \phi\right)_{i j k-\frac{1}{2}}\right), \\
(\mathbf{D} \cdot \mathbf{u})_{i j k} & =\frac{u_{1, i+\frac{1}{2} j k}-u_{1, i-\frac{1}{2} j k}}{\Delta x_{1}}+\frac{u_{2, i j+\frac{1}{2} k}-u_{2, i j-\frac{1}{2} k}}{\Delta x_{2}}+\frac{u_{3, i j k+\frac{1}{2}}-u_{3, i j k-\frac{1}{2}}}{\Delta x_{3}}, \\
(\mathbf{L} \phi)_{i j k} & =\frac{\phi_{i+1 j k}-2 \phi_{i j k}+\phi_{i-1 j k}}{\Delta x_{1}^{2}}+\frac{\phi_{i j+1 k}-2 \phi_{i j k}+\phi_{i j-1 k}}{\Delta x_{2}^{2}}+\frac{\phi_{i j k+1}-2 \phi_{i j k}+\phi_{i j k-1}}{\Delta x_{3}^{2}},
\end{aligned}
$$


onde $\mathbf{D}_{1}, \mathbf{D}_{\mathbf{2}}$ e $\mathbf{D}_{\mathbf{3}}$ são dados por

$$
\begin{aligned}
& \left(\mathbf{D}_{\mathbf{1}} \phi\right)_{i-\frac{1}{2} j k}=\frac{\phi_{i j k}-\phi_{i-1 j k}}{\Delta x_{1}}, \\
& \left(\mathbf{D}_{\mathbf{2}} \phi\right)_{i j-\frac{1}{2} k}=\frac{\phi_{i j k}-\phi_{i j-1 k}}{\Delta x_{2}} \mathrm{e} \\
& \left(\mathbf{D}_{\mathbf{3}} \phi\right)_{i j k-\frac{1}{2}}=\frac{\phi_{i j k}-\phi_{i j k-1}}{\Delta x_{3}} .
\end{aligned}
$$

Os operadores de interpolação do centro de uma célula computacional para a face $x_{2} x_{3}\left(\mathbf{I}_{x_{2} x_{3}}^{c \rightarrow f}\right)$ e da face $x_{2} x_{3}$ para o centro de uma célula computacional $\left(\mathbf{I}_{x_{2} x_{3}}^{f \rightarrow c}\right)$ são definidos respectivamente pelas expressões

$$
\begin{aligned}
\left(\mathbf{I}_{x_{2} x_{3}}^{c \rightarrow f} \phi\right)_{i+\frac{1}{2} j k} & =\frac{\phi_{i+1 j k}+\phi_{i j k}}{2}, \\
\left(\mathbf{I}_{x_{2} x_{3}}^{f \rightarrow c} u_{1}\right)_{i j k} & =\frac{u_{1, i+\frac{1}{2} j k}+u_{1, i-\frac{1}{2} j k}}{2} .
\end{aligned}
$$

Os operadores $\mathbf{I}_{x_{1} x_{3}}^{c \rightarrow f}, \mathbf{I}_{x_{1} x_{3}}^{f \rightarrow c}, \mathbf{I}_{x_{1} x_{2}}^{c \rightarrow f}$ e $\mathbf{I}_{x_{1} x_{2}}^{f \rightarrow c}$, relacionados às faces $x_{1} x_{3}$ e $x_{1} x_{2}$ são definidos de maneira similar.

A discretização do termo advectivo na equação da conservação da massa (3.8) por diferenças finitas centradas de segunda ordem não é estável. Strikwerda (2004) sugere o acréscimo de um termo de difusão numérica nesta equação para evitar tal instabilidade. Assim é obtida uma equação da conservação da massa modificada dada por

$$
\frac{\partial \rho}{\partial t}+\frac{\partial\left(\rho u_{j}\right)}{\partial x_{j}}=\epsilon \frac{\partial^{2} \rho}{\partial x_{j}^{2}},
$$

onde $\epsilon=O(\Delta x)$ e $\Delta x=\min \left\{\Delta x_{1}, \Delta x_{2}, \Delta x_{3}\right\}$. A discretização por diferenças finitas centradas de segunda ordem gera oscilações numéricas que tornam este método instável quando aplicado aos termos advectivos das equações do transporte da fração de mistura (2.62) e da conservação da massa modificada (3.39) (quando o termo advectivo domina o termo difusivo), conforme descrevem Strikwerda (2004), Trottenberg et al. (2001) e Versteeg e Malalasekera (2007). Para verificar se a equação possui termo advectivo dominante é necessário analisar o número de Péclet de malha. O número de Péclet $(P e)$ é a razão entre a taxa de advecção de uma propriedade física do escoamento e a taxa de difusão, definido por

$$
P e=\frac{L u^{*}}{D^{*}},
$$

onde $L$ é o comprimento característico do escoamento, $D^{*}$ é o coeficiente de difusão característico do escoamento e $u^{*}$ é a velocidade característica do escoamento. Desta forma, o número de Péclet fornece a razão entre a escala de tempo difusiva $\frac{L^{2}}{\rho^{*} D^{*}}$ e a escala de tempo advectiva $\frac{L}{u^{*} \rho^{*}}$. Se $P e \ll 1$, então a advecção é dominada pela difusão, sendo o transporte essencialmente afetado pela difusão. Se $P e \gg 1$, então a advecção domina a difusão. A seguinte inequação é conhecida como a condição de Péclet

$$
\frac{\Delta x}{D} \max \left(u_{1}, u_{2}, u_{3}\right) \leq 2 .
$$

Se a condição de Péclet é satisfeita, então a aproximação por diferenças finitas centradas fornece uma aproximação estável para o termo advectivo $\left(O\left(\Delta x^{2}\right)\right)$. Caso contrário, pode-se es- 
perar complicações numéricas como oscilações e instabilidade. Para contornar esta dificuldade Trottenberg et al. (2001) sugere a utilização de um esquema que combina diferenças finitas de primeira e segunda ordem ou um esquema de alta ordem para o termo advectivo.

No presente trabalho, utiliza-se o esquema QUICK (Quadratic Upstream Interpolation for Convective Kinetics) que possui terceira ordem para aproximar o termo advectivo. Para mais detalhes veja os trabalhos de Versteeg e Malalasekera (2007) e Ferziger e Perić (1999). Considere o termo advectivo da equação (3.39). A Figura 3.2 mostra as aproximações quadráticas de uma variável escalar, do centro para as faces de uma célula computacional, utilizadas pelo esquema QUICK.

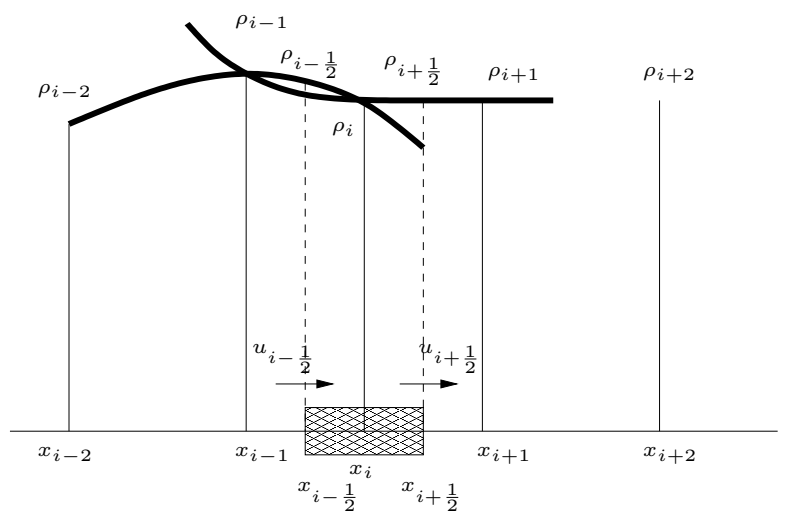

Figura 3.2: Aproximações quadráticas utilizadas pelo esquema QUICK em uma dimensão.

A aproximação da massa específica $\rho$, nas faces de uma célula computacional na direção $x_{1}$, é obtida por meio de uma interpolação quadrática dada por,

$$
\begin{gathered}
\rho_{i+\frac{1}{2} j k}= \begin{cases}\frac{6}{8} \rho_{i j k}+\frac{3}{8} \rho_{i+1 j k}-\frac{1}{8} \rho_{i-1 j k}, & \text { se } u_{i+\frac{1}{2} j k} \geq 0, \\
\frac{6}{8} \rho_{i+1 j k}+\frac{3}{8} \rho_{i j k}-\frac{1}{8} \rho_{i+2 j k}, & \text { caso contrário },\end{cases} \\
\rho_{i-\frac{1}{2} j k}= \begin{cases}\frac{6}{8} \rho_{i-1 j k}+\frac{3}{8} \rho_{i j k}-\frac{1}{8} \rho_{i-2 j k}, & \text { se } u_{i-\frac{1}{2} j k} \geq 0, \\
\frac{6}{8} \rho_{i j k}+\frac{3}{8} \rho_{i-1 j k}-\frac{1}{8} \rho_{i+1 j k}, & \text { caso contrário } .\end{cases}
\end{gathered}
$$

A aproximação é análoga para os valores de $\rho$ calculados nas faces nas direções $x_{2}$ e $x_{3}$. Tendo as aproximações da propriedade $\rho$ nas faces da célula computacional, a aproximação do termo advectivo é obtida por meio da expressão,

$$
\begin{aligned}
(\mathbf{D} u \rho)_{i j k}= & \frac{u_{i+\frac{1}{2} j k} \rho_{i+\frac{1}{2} j k}-u_{i-\frac{1}{2} j k} \rho_{i-\frac{1}{2} j k}}{\Delta x_{1}}+\frac{u_{i j+\frac{1}{2} k} \rho_{i j+\frac{1}{2} k}-u_{i j-\frac{1}{2} k} \rho_{i j-\frac{1}{2} k}}{\Delta x_{2}}+ \\
& +\frac{u_{i j k+\frac{1}{2}} \rho_{i j k+\frac{1}{2}}-u_{i j k+\frac{1}{2}} \rho_{i j k-\frac{1}{2}}}{\Delta x_{3}} .
\end{aligned}
$$




\subsubsection{Método Multigrid}

Uma vez feitas as discretizações espacial e temporal, a extensão do método da projeção requer a solução de doze sistemas lineares por passo de integração (seis para $k=1$ e seis para $k=2$ ). A solução destes sistemas lineares é feita por meio do método Multigrid-Multinível (descrito no Capítulo 4). Para estender o código AMR3D (veja Nós (2007)) para um escoamento reativo com baixo número de Mach, foi necessário acrescentar a resolução de uma equação de transporte para uma variável escalar localizada no centro de uma célula computacional. A resolução do sistema linear originado pela discretização desta equação de transporte é descrita brevemente. Detalhes dos métodos multigrid para as componentes das velocidades localizadas nas faces da célula computacional são encontrados em Nós (2007).

A ideia básica dos métodos multigrid consiste na combinação de dois princípios: suavizar as altas frequências do erro e calcular uma correção para a solução em uma malha mais grossa.

Considere o sistema linear:

$$
A_{l b} \phi_{l b}=f_{l b}
$$

obtido a partir da discretização de uma equação diferencial parcial em um domínio computacional $\Omega_{l b}$, com espaçamento $\Delta x_{1, l b}, \Delta x_{2, l b}, \Delta x_{3, l b}$ e uma sequência de malhas cada vez mais grossas $\Omega_{l b-l}$ com $l=1,2, \ldots n v l$, onde $n v l=l b-1$ é o número de níveis virtuais, isto é, as malhas que estão abaixo do nível físico $l b, A_{l b}$ é o operador contínuo discretizado, $\phi_{l b}$ é a solução exata e $f_{l b}$ é o lado direito do problema na malha $\Omega_{l b}$.

O erro na malha $\Omega_{l b}$ é definido por $\mathbf{e}_{l b}=\phi_{l b}-\tilde{\phi}_{l b}$, onde $\tilde{\phi}_{l b}$ é uma solução aproximada do sistema linear (3.45). Desta forma, a solução aproximada $\tilde{\phi}_{l b}$, pode ser vista como uma correção da solução $\phi_{l b}$, ou seja, $\phi_{l b}=\tilde{\phi}_{l b}+\mathbf{e}_{l b}$. Assim, pode-se reescrever o sistema linear (3.45) substituindo $\phi_{l b}$ por $\tilde{\phi}_{l b}+\mathbf{e}_{l b}$ e obtendo a equação residual,

$$
A_{l b} \mathbf{e}_{l b}=\mathbf{r}_{l b},
$$

sendo $\mathbf{r}_{l b}=f_{l b}-A \tilde{\phi}_{l b}$ o resíduo do sistema linear (3.45). Se $\mathbf{e}_{l b}$ for suave, então pode-se aproximá-lo na malha progressivamente mais grossa, $\Omega_{l b-1}$, e assim sucessivamente, até a malha mais grossa $\Omega_{1}$. Desta forma, obtém-se a equação residual na malha mais grossa, dada por

$$
A_{1} \mathbf{e}_{1}=\mathbf{r}_{1}
$$

Para resolver o sistema (3.45) são necessários operadores que transmitam as informações entre as malhas finas e grossas. Para cada nível $l=2, \ldots, l b$, considere $\mathcal{R}_{l}^{l-1}$ o operador de restrição que transfere informações da malha $\Omega_{l}$ para a malha $\Omega_{l-1}$ e $\mathcal{P}_{l-1}^{l}$ o operador de prolongamento que transfere informações da malha $\Omega_{l-1}$ para a malha $\Omega_{l}$. Em cada nível, um método iterativo, como por exemplo, o método de Gauss-Seidel, é utilizado para resolver o sistema (3.46). O objetivo é suavizar as altas frequências do erro neste nível. Na malha mais grossa $\Omega_{1}$ o sistema (3.47) deve ser resolvido exatamente. Numericamente isto significa que o método iterativo utilizado para suavizar o erro nas malhas mais finas é aplicado um número maior de vezes na malha mais grossa. Muitos métodos de relaxação eliminam as componentes de alta frequência do erro, mas não conseguem eliminar as componentes de baixa frequência. Esta característica é conhecida como uma propriedade de suavização. Por isso, um bom método de relaxação torna o método multigrid 
eficiente já que as baixas frequências do erro em uma malha são vistas como altas frequências em uma malha progressivamente mais grossa (para mais detalhes veja Briggs e McCormick (2000)).

Existem muitos operadores de prolongamento, restrição e suavização que podem ser empregados nos métodos multigrid. Os operadores de prolongamento e de restrição dependem da localização das variáveis no domínio computacional, Trottenberg et al. (2001) apresenta muitos exemplos de operadores tanto de restrição e prolongamento quanto de relaxação. No presente trabalho, utiliza-se o método de Gauss-Seidel preto-vermelho para a suavização.

O operador de restrição padrão utilizado, para as variáveis localizadas no centro de uma célula computacional, é descrito por meio da expressão

$$
\phi_{l-1}=\frac{1}{8} \sum_{m=1}^{8} \phi_{l, m}, \quad \text { para } l=2,3, \ldots, l b .
$$

A Figura 3.3 mostra o operador de interpolação para uma variável localizada no centro de uma célula computacional, descrito pela expressão (3.48).

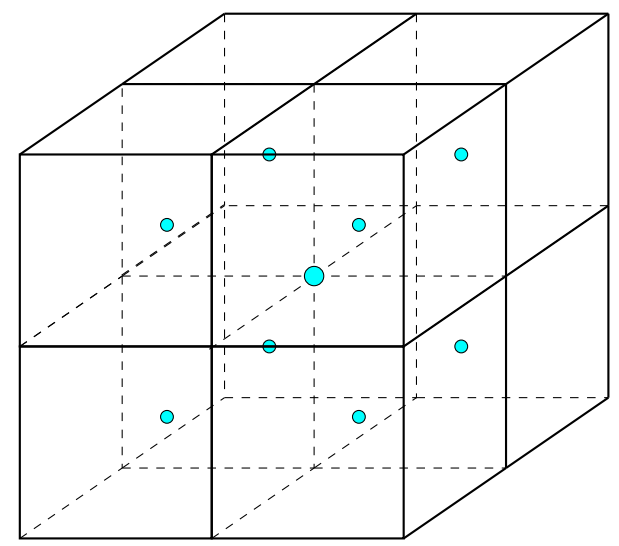

$$
\begin{array}{rr}
\phi_{l-1} & \circ \\
\phi_{l} & \circ
\end{array}
$$

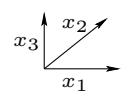

Figura 3.3: Restrição para uma variável escalar calculada no centro de uma célula computacional.

O operador de prolongamento utilizado para as variáveis localizadas no centro das células computacionais é dado por

$$
\begin{aligned}
\phi_{l} & =\left(1-t_{1}\right)\left(1-t_{2}\right)\left(1-t_{3}\right) \phi_{l-1,1}+t_{1}\left(1-t_{2}\right)\left(1-t_{3}\right) \phi_{l-1,2}+ \\
& +t_{1} t_{2}\left(1-t_{3}\right) \phi_{l-1,3}+\left(1-t_{1}\right) t_{2}\left(1-t_{3}\right) \phi_{l-1,4}+ \\
& +\left(1-t_{1}\right)\left(1-t_{2}\right) t_{3} \phi_{l-1,5}+t_{1}\left(1-t_{2}\right) t_{3} \phi_{l-1,6}+ \\
& +t_{1} t_{2} t_{3} \phi_{l-1,7}+\left(1-t_{1}\right) t_{2} t_{3} \phi_{l-1,8}, \quad \text { para } l=2,3, \ldots, l b,
\end{aligned}
$$

onde $t_{1}, t_{2}$ e $t_{3}$ definem os pesos, que podem valer $\frac{1}{4}$ ou $\frac{3}{4}$. A Figura 3.4 mostra o operador de prolongamento descrito pela espressão (3.49).

$\mathrm{O}$ método multigrid ciclo $\mathrm{V}$ para uma malha uniforme é descrito pelo algoritmo 3.1. Para mais detalhes veja Nós (2007), Trottenberg et al. (2001) e Briggs e McCormick (2000). 


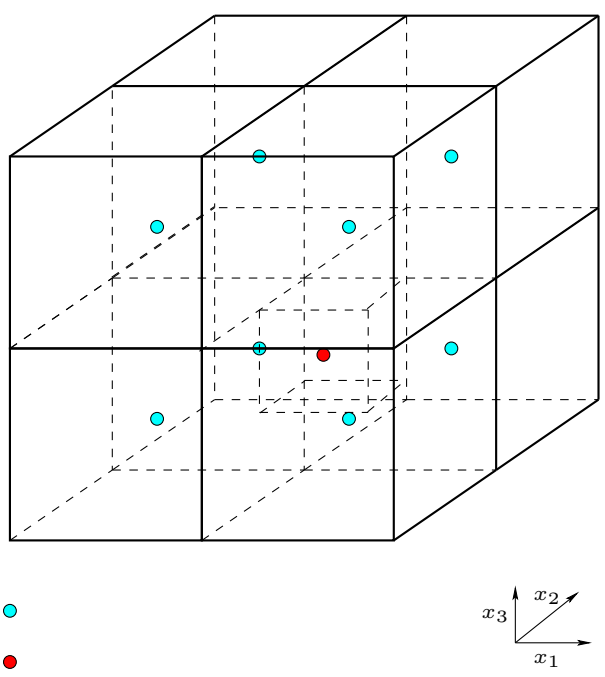

Figura 3.4: Prolongamento para uma variável calculada no centro da célula computacional.

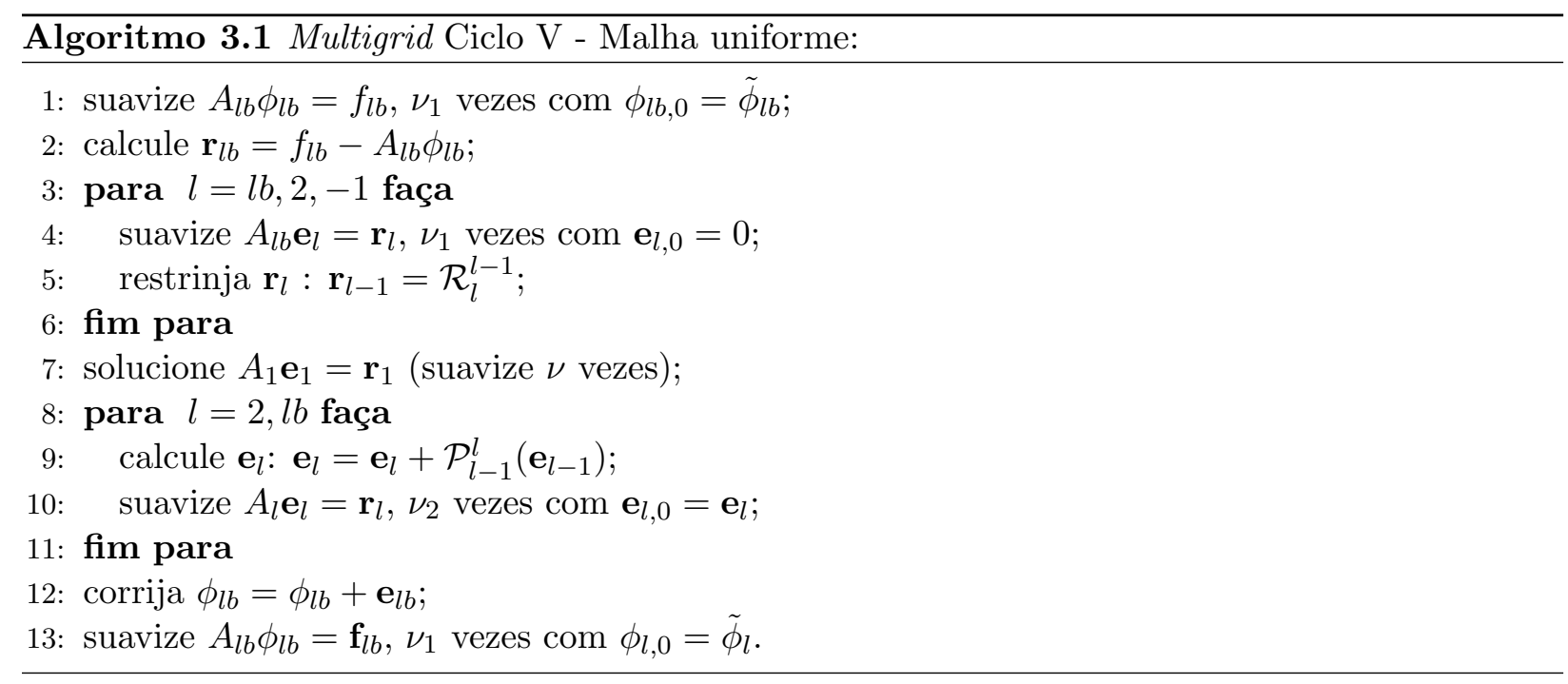

\subsubsection{Sumário da metodologia numérica para a fase contínua}

Para simplificar a descrição da metodologia numérica para a fase contínua, primeiramente, será descrita a versão estendida do método da Projeção. Com isso, os Algoritmos 3.2 e 3.3 descrevem um passo de integração da metodologia numérica para a simulação de um escoamento com baixo número de Mach e para um escoamento incompressível não-reativo.

O acoplamento pressão-velocidade presente na equação (3.23) é tratado com a extensão do método da projeção para escoamentos reativos com baixo número de Mach. No presente trabalho utiliza-se uma versão estendida baseada na metodologia numérica proposta por Pember et al. (1998). Além disso, faz-se uso da estratégia semi-implícita proposta por Bell et al. (1989), também aplicada por Roma (1996) e Nós (2007). Dados $\mathbf{u}^{n}, \mathbf{u}^{n-1}, p^{n}, p^{n-1}, \rho_{c}^{n}, \rho_{c}^{n-1}, S^{n+1}, \Delta t^{n-1}, \Delta t^{n}$, $\Delta t^{n+1}, \alpha_{2}, \theta_{2}$ e $\lambda$, a ideia da versão estendida do método da projeção, é descrita por meio dos seguintes passos. 
Passo 1. Determine o campo de velocidade auxiliar $\mathbf{u}^{\star}$ por meio da equação

$$
\begin{aligned}
\frac{\alpha_{2} u_{i}^{\star}+\alpha_{1} u_{i}^{n}+\alpha_{0} u_{i}^{n-1}}{\Delta t^{n+1}} & =\theta_{2}\left(\frac{\lambda}{\rho_{c}^{n+1}} \frac{\partial^{2} u_{i}^{\star}}{\partial x_{j}^{2}}-\frac{1}{\rho_{c}^{n+1}} \frac{\partial p_{2}^{n}}{\partial x_{i}}\right)+ \\
& +\theta_{1} \tilde{f}_{i}^{n}+\theta_{0} \tilde{f}_{i}^{n-1}+\beta_{1} \tilde{g}_{i}^{n}+\beta_{0} \tilde{g}_{i}^{n-1}
\end{aligned}
$$

para $i=1,2,3$ e com $\tilde{f}_{i}$ e $\tilde{g}_{i}$ dadas, respectivamente, pelas expressões (3.24) e (3.22).

Passo 2. Projete $\mathbf{u}^{\star}-\nabla \xi$ no espaço vetorial dos campos com divergente nulo, sendo que $\xi$ satisfaz $\nabla \cdot \nabla \xi=\nabla \mathbf{u}^{n+1}$. Isto é necessário pois $\nabla \cdot \mathbf{u}^{\star} \neq S^{n+1}$. Desta maneira, $\mathbf{u}^{\star}$ pode ser decomposto como segue

$$
\mathbf{u}^{\star}=\mathbf{u}^{n+1}+\frac{\Delta t^{n+1} \theta_{2}}{\alpha_{2} \rho_{c}^{n+1}} \nabla \phi
$$

sendo $\mathbf{u}^{n+1}=\mathbf{u}_{d}+\nabla \xi, \nabla \cdot \mathbf{u}^{n+1}=S^{n+1}, \nabla \cdot \mathbf{u}_{d}=0, \phi$ a correção para a pressão $p_{2}^{n}$ e para o campo de velocidade $\mathbf{u}^{n+1}$.

Passo 3. Aplique o operador de projeção, $\mathcal{P}\left(\mathbf{u}^{\star}-\nabla \xi\right)=\mathbf{u}_{d}$. Isso é feito substituindo-se a expressão (3.51) na equação (3.50), obtendo-se

$$
\begin{aligned}
\frac{\alpha_{2} u_{i}^{n+1}+\alpha_{1} u_{i}^{n}+\alpha_{0} u_{i}^{n-1}}{\Delta t^{n+1}} & =-\frac{\theta_{2}}{\rho_{c}^{n+1}} \frac{\partial \phi}{\partial x_{i}}+\theta_{2}\left[\frac{\lambda}{\rho_{c}^{n+1}} \frac{\partial^{2} u_{i}^{n+1}}{\partial x_{j}^{2}}-\frac{1}{\rho_{c}^{n+1}} \frac{\partial p_{2}^{n}}{\partial x_{i}}+\right. \\
& \left.+\frac{\lambda \Delta t^{n+1}}{\alpha_{2} \rho_{c}^{n+1}} \frac{\partial}{\partial x_{j}}\left(\frac{1}{\rho_{c}^{n+1}} \frac{\partial \phi}{\partial x_{j}}\right)\right]+ \\
& +\theta_{1} \tilde{f}_{i}^{n}+\theta_{0} \tilde{f}_{i}^{n-1}+\beta_{1} \tilde{g}_{i}^{n}+\beta_{0} \tilde{g}_{i}^{n-1},
\end{aligned}
$$

com $i=1,2,3$ e $\tilde{f}_{i}$ e $\tilde{g}_{i}$ dadas, respectivamente, pelas expressões (3.24) e (3.22). Subtraindo a equação (3.52) da equação (3.23) obtém-se a expressão

$$
\frac{\partial p_{2}^{n+1}}{\partial x_{i}}=\frac{\partial p_{2}^{n}}{\partial x_{i}}+\frac{\partial \phi}{\partial x_{i}}-\frac{\lambda \Delta t^{n+1}}{\alpha_{2}} \frac{\partial}{\partial x_{j}}\left(\frac{1}{\rho_{c}^{n+1}} \frac{\partial \phi}{\partial x_{j}}\right)
$$

Como observado por Nós (2007), ao desprezar o último termo do lado direito da expressão (3.53), obtém-se uma aproximação de pelo menos primeira ordem para a pressão por meio da expressão

$$
\frac{\partial p_{2}^{n+1}}{\partial x_{i}}=\frac{\partial p_{2}^{n}}{\partial x_{i}}+\frac{\partial \phi}{\partial x_{i}}
$$

Passo 4. Resolva a equação que determina a correção $\phi$. Para isso basta aplicar o operador $\nabla \cdot$ na equação $(3.51)$ obtendo-se

$$
\nabla \cdot\left(\frac{1}{\rho_{c}^{n+1}} \nabla \phi\right)=\frac{\alpha_{2}}{\theta_{2} \Delta t^{n+1}}\left(\nabla \cdot \mathbf{u}^{\star}-S^{n+1}\right)
$$


ou na notação indicial,

$$
\frac{\partial}{\partial x_{j}}\left(\frac{1}{\rho_{c}^{n+1}} \frac{\partial \phi}{\partial x_{j}}\right)=\frac{\alpha_{2}}{\theta_{2} \Delta t^{n+1}}\left(\frac{\partial u_{j}^{\star}}{\partial x_{j}}-S^{n+1}\right) .
$$

Após determinar a correção $\phi$, a pressão e o campo de velocidade são corrigidos, respectivamente, por

$$
\begin{aligned}
& p_{2}^{n+1}=p_{2}^{n}+\phi \\
& u_{i}^{n+1}=u_{i}^{\star}-\frac{\Delta t^{n+1} \theta_{2}}{\alpha_{2} \rho_{c}^{n+1}} \frac{\partial \phi}{\partial x_{i}}, \quad \text { para } i=1,2,3 .
\end{aligned}
$$

As condições de fronteira para a equação elíptica (3.55) são obtidas a partir das condições de fronteira impostas para o campo de velocidade $\mathbf{u}^{n+1}$. O uso das condições de fronteira Dirichlet para o campo de velocidade $\mathbf{u}^{n+1}$ implica em condições de fronteira homogêneas de Neumann para a correção $\phi$. Vale ressaltar que neste caso, é necessário satisfazer a condição de compatibilidade dada por

$$
\int_{\Omega} \frac{\alpha_{2}}{\Delta t} \nabla \cdot \mathbf{u}^{\star} d \Omega=\int_{\partial \Omega} \frac{\partial}{\partial \mathbf{n}} \phi^{n+1} d S=0,
$$

que garante a unicidade da solução do sistema linear obtido a partir da discretização da equação (3.55). Para mais detalhes veja Thomas (1995) e Hackbusch (1992).

O Algoritmo 3.2 descreve um passo de integração para um escoamento reativo com baixo número de Mach. No início de cada passo de integração é assumido que os valores para $\Delta t^{n-1}, \Delta t^{n}, \mathbf{u}^{n-1}$, $\mathbf{u}^{n}, p_{2}^{n-1}, p_{2}^{n}, \mu^{n-1}, \mu^{n}, \rho_{c}^{n-1}, \rho_{c}^{n}, z^{n-1}, z^{n}, D^{n-1}, D^{n}, Y_{l}^{n-1}, Y_{l}^{n}, T^{n-1}$ e $T^{n}$ são conhecidos.

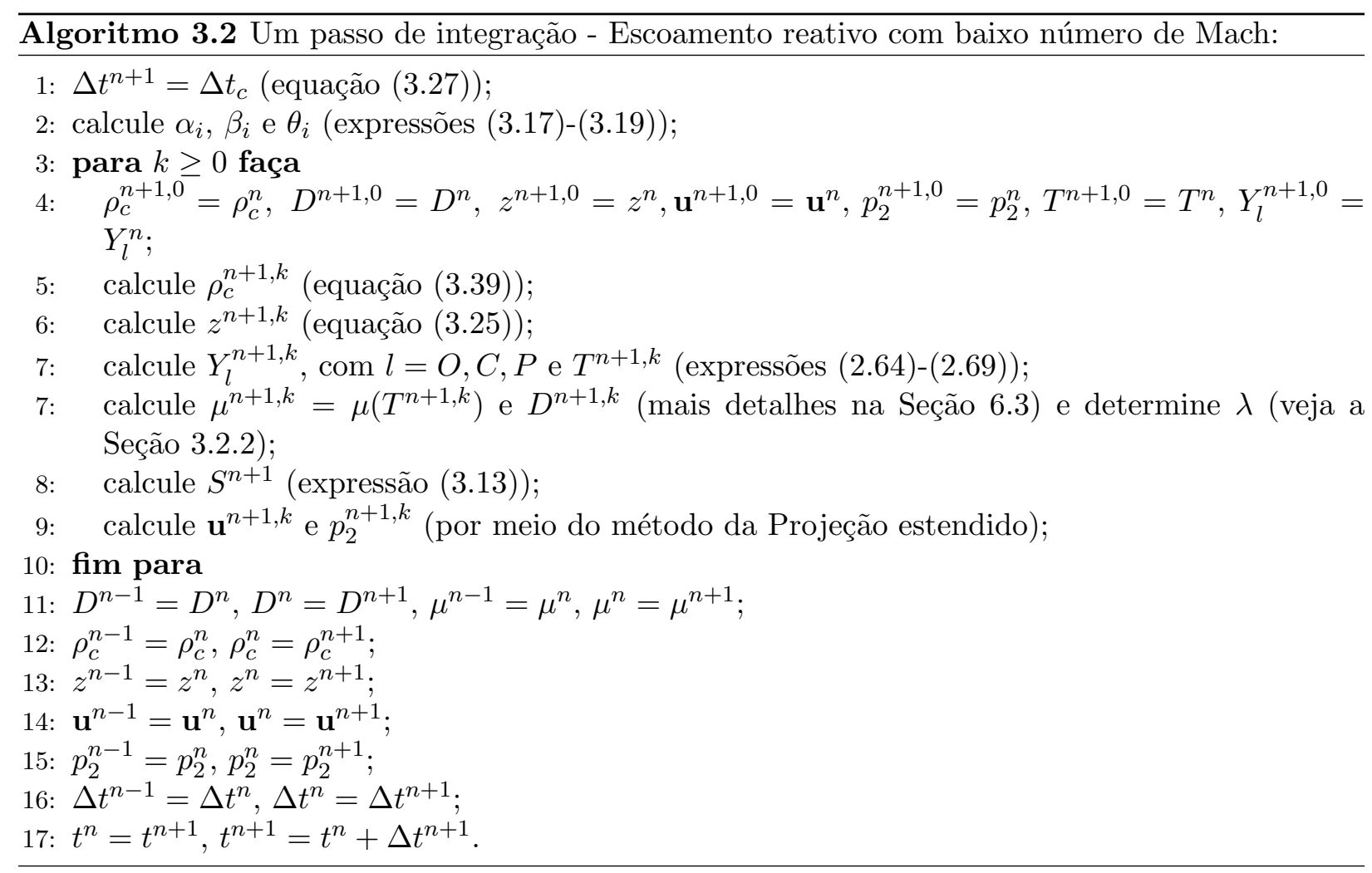


O termo $S^{n+1}$, dado pela expressão (3.13), no Algoritmo 3.2 é aproximado por meio da expressão

$$
\begin{aligned}
S^{n+1} & =\frac{1}{\left(\rho_{c} T\right)^{n+1}}\left[\frac{\alpha_{2}\left(\rho_{c} T\right)^{n+1}+\alpha_{1}\left(\rho_{c} T\right)^{n}+\alpha_{0}\left(\rho_{c} T\right)^{n-1}}{\Delta t^{n+1}}+\beta_{1}\left(\frac{\partial \rho_{c} u_{i} T}{\partial x_{i}}\right)^{n}+\right. \\
& \left.+\beta_{0}\left(\frac{\partial \rho_{c} u_{i} T}{\partial x_{i}}\right)^{n-1}\right]+\frac{W}{\rho_{c}^{n+1}} \sum_{l}\left[\frac { 1 } { W _ { l } } \left(\frac{\alpha_{2}\left(\rho_{c} Y_{l}\right)^{n+1}+\alpha_{1}\left(\rho_{c} Y_{l}\right)^{n}+\alpha_{0}\left(\rho_{c} Y_{l}\right)^{n-1}}{\Delta t^{n+1}}+\right.\right. \\
& \left.\left.+\beta_{1}\left(\frac{\partial \rho_{c} u_{i} Y_{l}}{\partial x_{i}}\right)^{n}+\beta_{0}\left(\frac{\partial \rho_{c} u_{i} Y_{l}}{\partial x_{i}}\right)^{n-1}\right)\right], \quad \text { com } l=C, O, P .
\end{aligned}
$$

Note que a iteração em $k$ é necessária para separar a solução em duas partes, uma para as componentes da velocidade e uma para a correção da pressão. Além disso, esta iteração atualiza a viscosidade $\mu$ e o coeficiente de difusão $D$. No presente trabalho duas iterações garantiram a convergência de metodologia.

O Algoritmo 3.3 descreve um passo de integração para um escoamento incompressível nãoreativo. No início de cada passo de integração assume-se que os valores para $\Delta t^{n-1}, \Delta t^{n}, \mathbf{u}^{n-1}$, $\mathbf{u}^{n}, p_{2}^{n-1}, p_{2}^{n}, \mu^{n-1}, \mu^{n}$ são conhecidos. Para o caso incompressível, o termo $S^{n+1}$ na versão estendida do método da Projeção é nula, além disso, no presente trabalho, $\rho_{c}$ é constante.

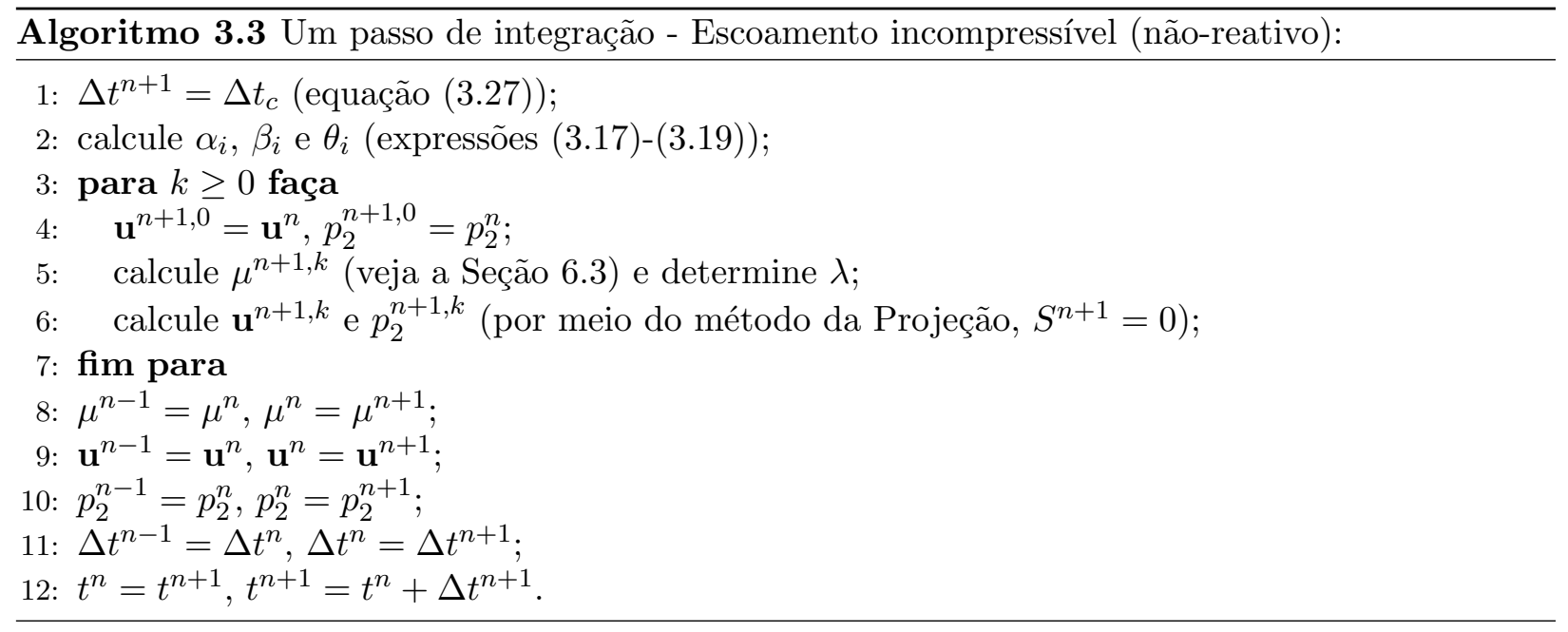

\subsection{Solução das equações que modelam a fase dispersa}

$\mathrm{Na}$ formulação lagrangiana da fase dispersa, cada gotícula é representada no domínio computacional por meio da sua posição $\mathbf{X}_{p}(t)$. A Figura 3.5 mostra uma célula computacional de uma malha euleriana com a representação lagrangiana das gotículas, a posição de um campo de velocidade $\mathbf{u}=\left(u_{1}, u_{2}, u_{3}\right)$ e a posição de uma variável escalar $\phi$.

As propriedades da fase dispersa são modeladas por meio das equações descritas no Capítulo 2 para cada gotícula. Denota-se por $N_{p}$ o número de gotículas presentes na simulação numérica. 

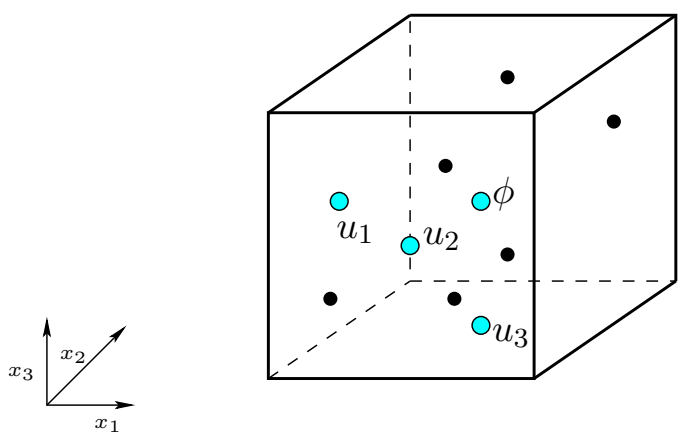

Figura 3.5: Localização de uma variável escalar $\phi$ e uma vetorial $\mathbf{u}=\left(u_{1}, u_{2}, u_{3}\right)$ discretizadas, respectivamente no centro e nas faces de uma célula computacional e as gotículas lagrangianas, representadas pelo símbolo $\bullet$, contidas nesta célula.

Associado a cada gotícula tem-se o seguinte sistema de equações diferenciais ordinárias

$$
\begin{aligned}
& \frac{d \mathcal{Y}_{p}(t)}{d t}=\mathcal{F}\left(t, \mathcal{Y}_{p}\right), t \in\left[t_{0}, \bar{t}\right], \\
& \mathcal{Y}_{p}\left(t_{0}\right)=\mathcal{Y}_{p}^{0}, \text { para } 1 \leq p \leq N_{p},
\end{aligned}
$$

sendo $t_{0}$ o tempo inicial da simulação, $\bar{t}$ o tempo final da simulação, $\mathcal{Y}_{p}=\left[\mathbf{X}_{p}, \mathbf{U}_{p}\right]^{T}$ e $\mathcal{F}$ representa o lado direito das equações, o qual pode envolver propriedades vindas da fase contínua do escoamento.

O tamanho do passo no tempo para a fase dispersa é dado por

$$
\Delta t_{d}=C \min \left(\frac{\Delta x_{1}}{\left|u_{1, p}\right|_{\max }}, \frac{\Delta x_{2}}{\left|u_{2, p}\right|_{\max }}, \frac{\Delta x_{3}}{\left|u_{3, p}\right|_{\max }}\right), \text { com } 0<C<1 \quad \text { e } 0 \leq p \leq N_{p} .
$$

\subsubsection{Discretização temporal da fase dispersa}

A discretização temporal das equações que modelam a fase dispersa na formulação lagrangiana é feita por meio do método de Euler Modificado. O método de Euler Modificado é um método de passo único de segunda ordem de aproximação. Ao aplicar tal método ao sistema (3.60) obtém-se

$$
\left\{\begin{array}{l}
\mathcal{Y}_{p}^{n+1}=\mathcal{Y}_{p}^{n}+\frac{\Delta t}{2}\left(\kappa_{1}+\kappa_{2}\right), \\
\mathcal{Y}_{p}^{0}=\mathcal{Y}_{p}\left(t_{0}\right), \\
\kappa_{1}=\mathcal{F}\left(t^{n}, \mathcal{Y}_{p}^{n}\right), \\
\kappa_{2}=\mathcal{F}\left(t^{n}+\Delta t, \mathcal{Y}_{p}^{n}+\Delta t \kappa_{1}\right),
\end{array}\right.
$$

onde $p$ e $n$ são inteiros tais que $1 \leq p \leq N_{p}, n \geq 0$ e $\mathcal{Y}_{p}^{n} \approx \mathcal{Y}_{p}\left(t^{n}\right)$. Mais detalhes sobre o método de Euler Modificado podem ser encontrados em Burden e Faires (2005) e Stoer e Bulirsch (2002).

\subsubsection{Operadores de interpolação entre as fases contínua e dispersa}

Para o acoplamento entre as fases contínua e dispersa são necessários operadores de interpolação que levem as variáveis representadas na formulação euleriana para a posição das variáveis repre- 
sentadas na formulação lagrangiana e vice-versa. Os termos responsáveis pelo acoplamento entre as fases são descritos na Seção 2.5. O operador de interpolação no sentido fase dispersa-contínua é chamado operador de espalhamento. Os operadores de interpolação e espalhamento que determinam o acoplamento entre as fases contínua e dispersa são uma aproximação suave da função Delta de Dirac $\left(\delta_{\Delta x}\left(\mathbf{x}_{i j k}-\mathbf{X}_{p}\right)\right)$ conforme descrevem Griffith (2005) e Roma (1996). Tal função é dada pela expressão

$$
\delta_{\Delta x}\left(\mathbf{x}_{i j k}-\mathbf{X}_{p}\right)=\delta_{\Delta x_{1}}\left(x_{1, i j k}-X_{p, 1}\right) \delta_{\Delta x_{2}}\left(x_{2, i j k}-X_{p, 2}\right) \delta_{\Delta x_{3}}\left(x_{3, i j k}-X_{p, 3}\right),
$$

onde $1 \leq p \leq N_{p}, \mathbf{x}_{i j k}$ e $\mathbf{X}_{p}$ representam, respectivamente, a posição de uma variável no domínio computacional e a posição da gotícula lagrangiana $p$. Uma aproximação suave para a função Delta de Dirac unidimensional, é dada por

$$
\delta_{\Delta x_{i}}\left(x_{i}-X_{p, i}\right) \approx \frac{1}{\Delta x_{i}} \psi\left(\frac{x_{i}-X_{p, i}}{\Delta x_{i}}\right), \text { para } i=1,2,3,
$$

onde a função $\psi$ é

$$
\psi(r)= \begin{cases}\frac{1}{8}\left(3-2|r|+\sqrt{1+4|r|-4 r^{2}}\right), & \text { se } 0 \leq|r|<1, \\ \frac{1}{8}\left(5-2|r|-\sqrt{12|r|-7-4 r^{2}}\right), & \text { se } 1 \leq|r|<2, \\ 0, & \text { se } 2 \leq|r| .\end{cases}
$$

Essa função é determinada pela imposição das seguintes condições:

1. $\psi(r)$ é contínua para todo número real $r$,

2. $\psi(r)=0$ para $|r| \geq 2$,

3. $\sum_{j}(r-j) \psi(r-j)=0$, para todo $r$ tal que $-\infty<j<+\infty$,

4. $\sum_{j \text { pares }} \psi(r-j)=\sum_{j \text { ímpares }} \psi(r-j)=\frac{1}{2}$, para todo $r$,

5. $\sum_{j}[\psi(r-j)]^{2}=\frac{3}{8}$, para todo $r$.

Tanto o operador de interpolação quanto o operador de espalhamento são definidos pelas expressões (3.63)-(3.65). Para fazer o acoplamento entre as fases é necessário localizar as gotículas em cada uma das células do domínio computacional. A localização das gotículas em uma malha estruturada uniforme é imediata. Dada uma gotícula $p$ com $\mathbf{X}_{p}=\left(X_{p, 1}, X_{p, 2}, X_{p, 3}\right)$, os índices $(i, j, k)$, da célula computacional que contém a gotícula $p$, são obtidos por meio das expressões

$$
i=\left\lfloor\frac{X_{p, 1}-a_{1}}{\Delta x_{1}}\right\rfloor+1, \quad j=\left\lfloor\frac{X_{p, 2}-a_{2}}{\Delta x_{2}}\right\rfloor+1 \quad \text { e } \quad k=\left\lfloor\frac{X_{p, 3}-a_{3}}{\Delta x_{3}}\right\rfloor+1,
$$

onde $\Delta x_{1}, \Delta x_{2}$ e $\Delta x_{3}$ são os espaçamentos em cada uma das direções $a_{1}, a_{2}$ e $a_{3}$ são as coordenadas da posição inicial do domínio computacional e $\lfloor\cdot\rfloor$ é a parte inteira de um número real. 
Com isso, o espalhamento das forças que agem sobre uma gotícula em uma célula computacional $(i, j, k)$ é dado por

$$
S_{\mathbf{u}, p}=-\delta\left(\mathbf{x}_{i j k}-\mathbf{X}_{p}\right) \frac{d \mathbf{U}_{p}}{d t} .
$$

O espalhamento das forças que agem sobre todas as gotículas contidas em uma célula computacional é

$$
S_{\mathbf{u}}=\sum_{p=1}^{n_{p}} S_{\mathbf{u}, p},
$$

sendo $n_{p}$ o número de gotículas contidas na célula computacional $(i, j, k)$.

\subsubsection{Sumário da metodologia numérica para um escoamento incompressível contendo gotículas}

Para o escoamento incompressível contendo gotículas, a fase dispersa é aproximada por meio do método de Euler Modificado. O Algoritmo 3.4 descreve um passo de integração para um escoamento incompressível (não-reativo) com gotículas inertes. No início de cada passo de integração assume-se que os valores para $\Delta t^{n-1}, \Delta t^{n}, \mathbf{u}^{n-1}, \mathbf{u}^{n}, p_{2}^{n-1}, p_{2}^{n}, \mu^{n-1}, \mu^{n}, \mathbf{X}_{p}^{n}$ e $\mathbf{U}_{p}^{n}$ são conhecidos.

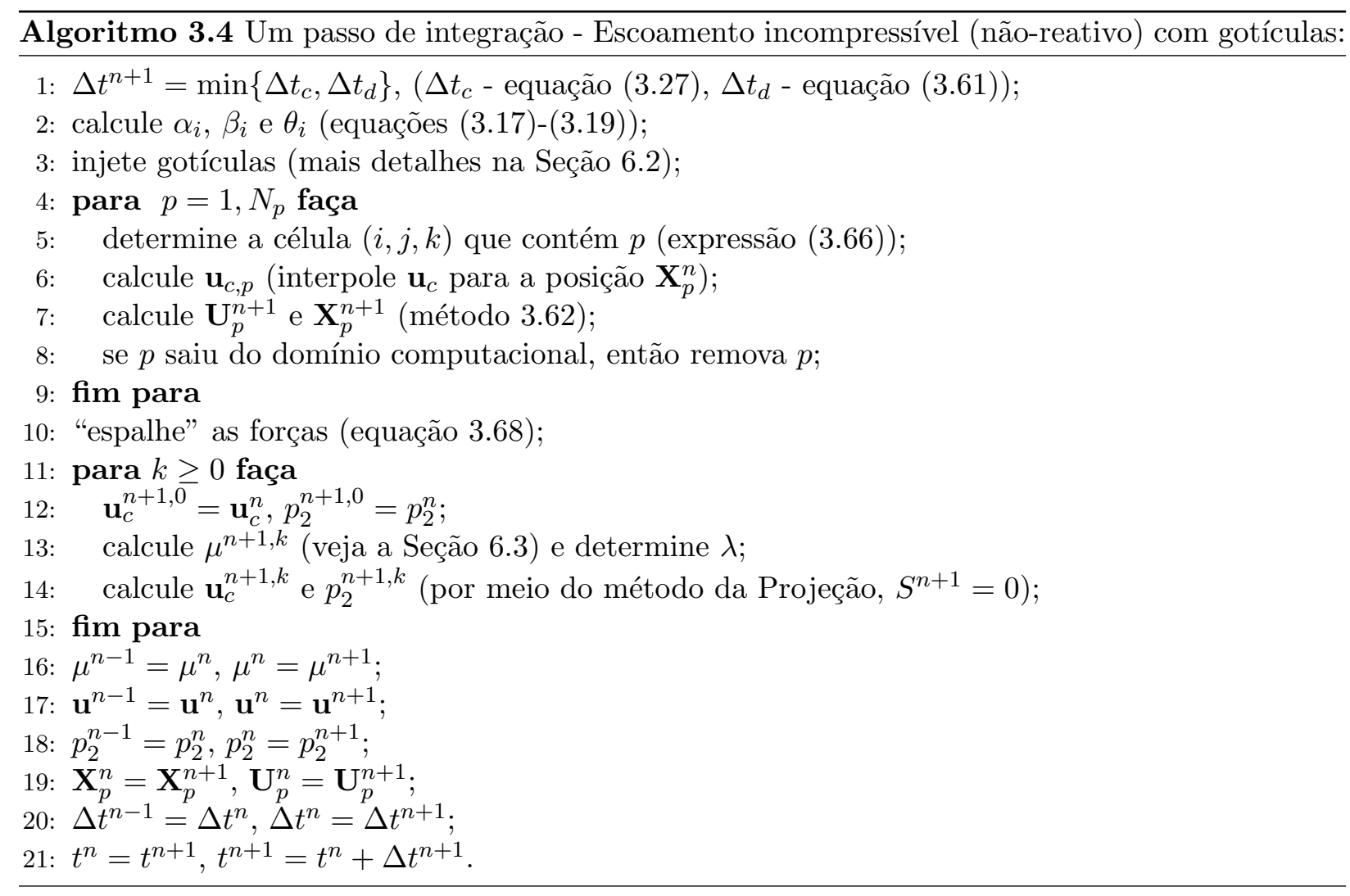

No Capítulo 4 é descrito a aplicação das metodologias numéricas descritas neste capítulo, considerando uma malha bloco-estruturada com refinamento adaptativo. No Capítulo 5 são apresentadas as verificações numéricas das metodologias propostas e no Capítulo 6 são apresentados os resultados numéricos. 


\subsection{Nomenclatura do presente capítulo}

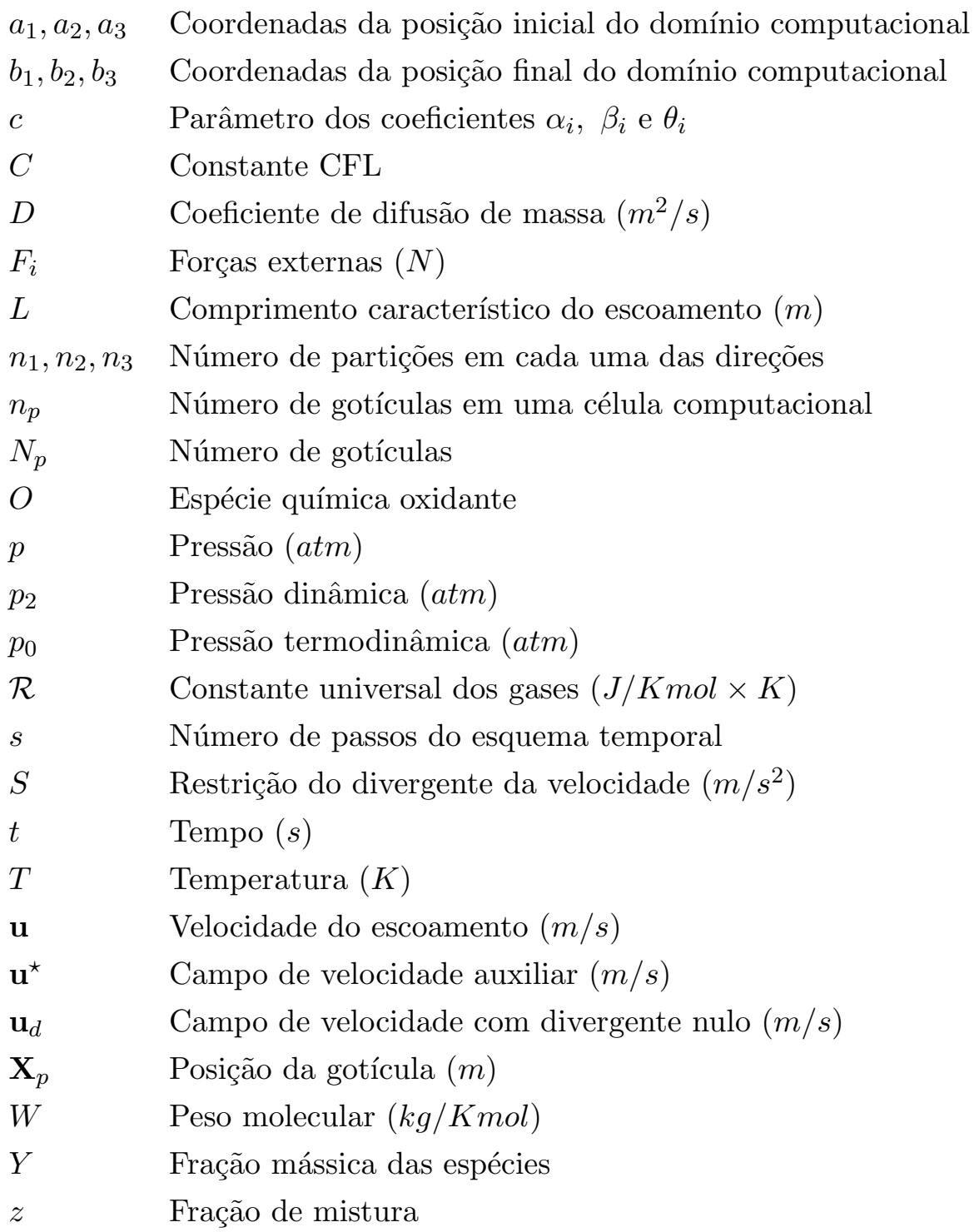

\section{Letras Gregas}

$\alpha_{i}, \theta_{i}$ e $\beta_{i} \quad$ Parâmetros dos esquemas temporais IMEX

$\delta \quad$ Delta de Dirac

$\Delta t \quad$ Tamanho do passo no tempo $(s)$

$\Delta x_{i} \quad$ Espaçamento na direção $i(m)$

$\epsilon \quad$ Coeficiente de difusão numérica

$\gamma \quad$ Parâmetro dos coeficientes $\alpha, \beta$ e $\theta$

$\kappa_{1}, \kappa_{2} \quad$ Parâmetros do método de Euler Modificado

$\mu \quad$ Viscosidade dinâmica $\left(N \times s / m^{2}\right)$

$\rho \quad$ Massa específica, densidade $\left(\mathrm{kg} / \mathrm{m}^{3}\right)$

$\Omega \quad$ Domínio computacional

$\phi \quad$ Correção da pressão e da velocidade

$\xi \quad$ Variável escalar

\section{Adimensionais}


Pe Número de Péclet Ma Número de Mach

\section{Índices}

$i, j, k \quad$ Direções das coordenadas $l \quad$ Espécie química

$p \quad$ Gotícula $n$ Tempo

* Valores característicos $\quad C$ Espécie química combustível

$P \quad$ Espécie química produto $O$ Espécie química oxidante

$c \quad$ Fase contínua $\quad d \quad$ Fase dispersa

\section{Operadores}

$\nabla \quad$ Gradiente

$\Delta \quad$ Laplaciano

$\nabla \cdot \quad$ Divergente

$\mathcal{P} \quad$ Projeção

$\lfloor\cdot\rfloor \quad$ Parte inteira de um número real

I Operador de Interpolação
G Gradiente Discreto

L Laplaciano Discreto

D Divergente Discreto

$\frac{D}{D t} \quad$ Derivada total

$\mathcal{I}$ Identidade

\section{Siglas}

IMEX Implícito-Explícito

CNAB Cranck Nicolson Adams-Bashforth

MCNAB Modified Cranck Nicolson Adams-Bashforth

CNLF Cranck Nicolson Leap Frog

CFL Courant-Friedrichs-Lewy

QUICK Quadratic Upstream Interpolation for Convective Kinetics 


\section{Capítulo 4}

\section{Malha adaptativa bloco-estruturada}

A adaptatividade é um conceito importante para a eficiência de métodos numéricos aplicados à resolução de equações diferenciais parciais. A principal vantagem da técnica de malhas adaptativas é concentrar boa resolução em regiões de interesse. A princípio, distinguem-se dois tipos de aproximação adaptativa: o refinamento estático, no qual as regiões de refinamento no domínio computacional são determinadas antes da execução do método numérico iniciar, e o refinamento dinâmico, no qual as regiões de refinamento são determinadas durante a execução do método numérico, controlado por algum critério de adaptatividade apropriado. Na prática, as duas aproximações podem ser combinadas.

Para a discretização do domínio computacional, o presente trabalho utiliza uma malha blocoestruturada com refinamento adaptativo e dinâmico baseando-se na estratégia de refinamento adaptativo de malhas, (Adaptative Mesh Refinement-AMR), proposta por Berger e Oliger (1984), Berger e Rigoutsos (1991) e Berger e Colella (1989).

A Seção 4.1 descreve o tipo e as características da malha adaptativa utilizada. A Seção 4.2 explica o procedimento de geração da malha com refinamento adaptativo, bem como os critérios de refinamento utilizados para os problemas de aplicação. A Seção 4.3 apresenta o tratamento das células computacionais fantasmas. A Seção 4.4 apresenta o método Multigrid-Multinível usado para resolver o sistema linear derivado da discretização espacial e temporal da equação do transporte da fração de mistura. O capítulo é encerrado com a apresentação da metodologia numérica com refinamento adaptativo de malhas para um escoamento incompressível (não-reativo) com gotículas inertes.

\subsection{Descrição de uma malha bloco-estruturada}

A descrição da malha bloco-estruturada utilizada no presente trabalho segue as descrições feitas por Berger e Colella (1989), Roma (1996) e Nós (2007). Uma malha bloco-estruturada é formada por um conjunto de blocos (paralelepípedos) discretizados em diferentes níveis de refinamento. As faces de cada bloco estão alinhadas com as faces dos eixos coordenados do domínio computacional. Cada bloco é um conjunto de células computacionais. Os blocos que compõem um nível de refinamento tem o mesmo espaçamento $\Delta x_{1}, \Delta x_{2}$ e $\Delta x_{3}$. A razão de refinamento $r$ entre o espaçamento de dois níveis de refinamento consecutivos é constante, de maneira que

$$
\Delta x_{i, l+1}=\frac{\Delta x_{i, l}}{r}, \text { para } i=1,2,3 .
$$


A Figura 4.1 apresenta uma malha bloco-estruturada tridimensional com a malha base e mais dois níveis de refinamento.
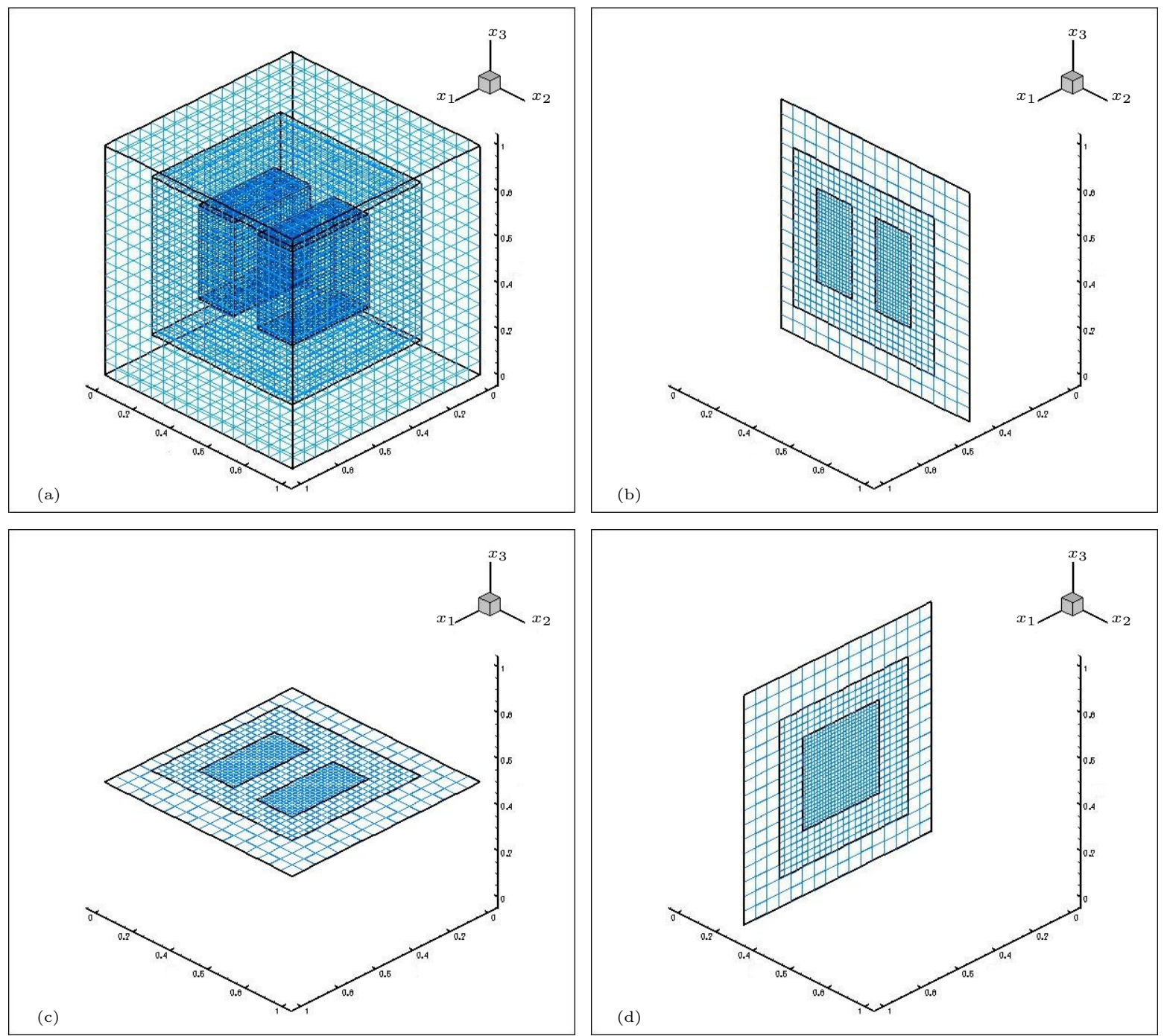

Figura 4.1: Malha bloco-estruturada em um domínio computacional $[0,1] \times[0,1] \times[0,1]$ com dois níveis de refinamento: (a) malha tri-dimensional, (b) corte na direção $x_{1}=0.42$, (c) corte na direção $x_{3}=0.45$ e (d) corte na direção $x_{2}=0.3$, Nós (2007).

Considere $G_{l, m}$ como sendo o $m$-ésimo bloco de um nível de refinamento $l$. Uma malha blocoestruturada deve atender àlgumas restrições. Uma restrição é que para cada dois blocos distintos de um nível de refinamento $l, G_{l, i}$ e $G_{l, j}$, tem-se que $G_{l, i} \cap G_{l, j}=\emptyset$ (exceto pelas faces). Assim, define-se para cada nível de refinamento $l$, a malha do nível $l$ por

$$
G_{l}=\bigcup_{k=1}^{n_{l}} G_{l, k},
$$

onde $n_{l}$ é o número de blocos do nível $l$. A partir desse ponto uma malha do nível $l$ é um conjunto de blocos discretizados no nível de refinamento $l$. Uma malha bloco-estruturada é a união das malhas de todos os níveis

$$
G=\bigcup_{l} G_{l}
$$

A Figura 4.2 mostra um exemplo de malha bloco-estruturada bidimensional. A Figura 4.2(a) 
mostra a malha do nível base. As Figuras 4.2(b) e 4.2(d) mostram as regiões cobertas por um nível de refinamento. As Figuras 4.2(c) e 4.2(e) mostram os blocos discretizados que formam cada nível de refinamento. A Figura 4.2(f) mostra a malha bloco-estruturada formada pela união dos três niveis de refinamento.

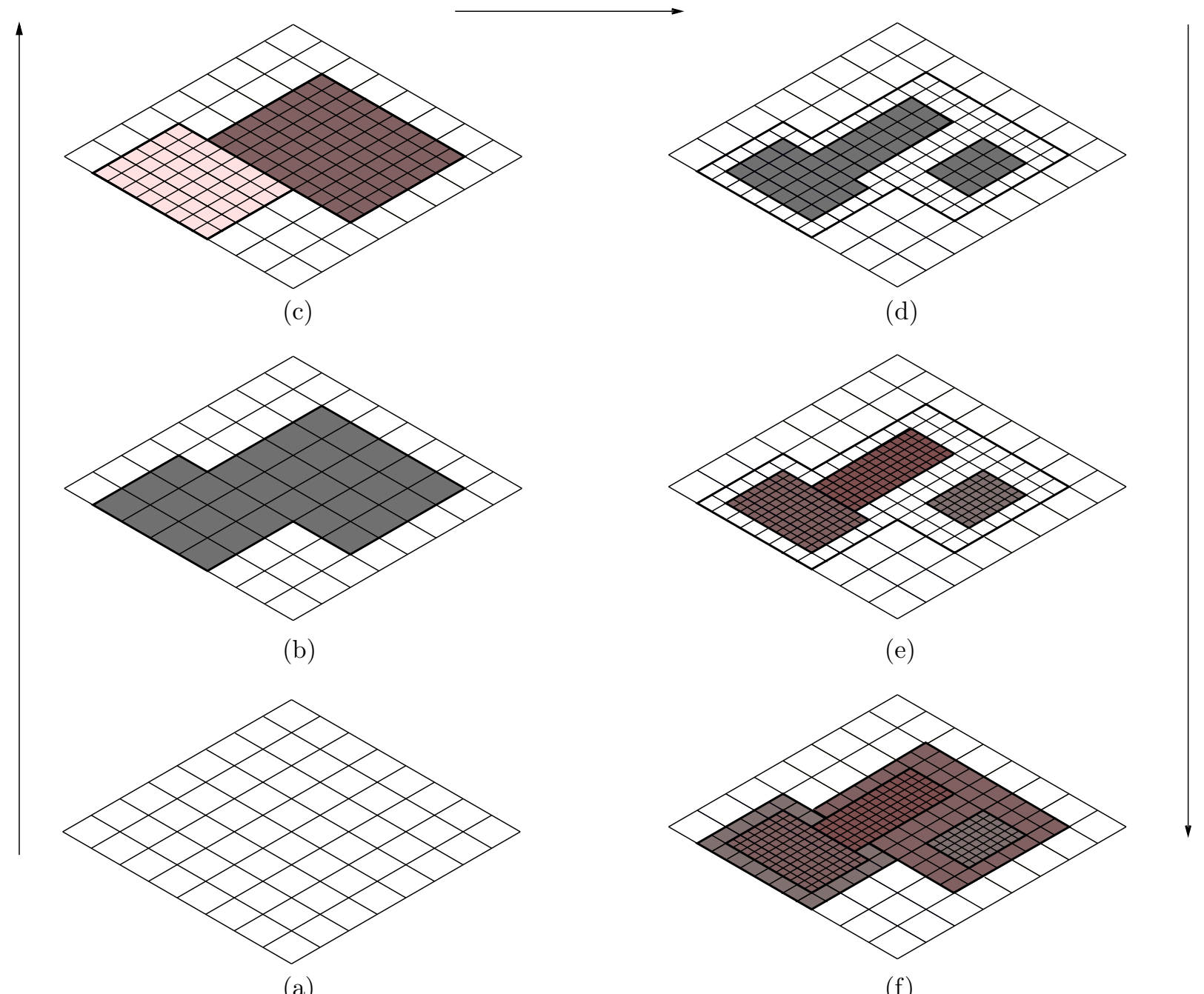

(a)

(f)

Figura 4.2: Elementos de uma malha bloco-estruturada bidimensional: (a) malha base; (b)-(d) regiões cobertas por um nível de refinamento; (c) nível de refinamento com dois blocos discretizados; (e) nível de refinamento com três blocos discretizados e (f) malha bloco-estruturada.

Outra restrição é que blocos em níveis diferentes adjacentes precisam estar propriamente aninhados, ou seja,

1. um bloco em um nível fino deve começar e terminar no canto de uma célula computacional do próximo nível mais grosso; e

2. para cada canto de um bloco do nível fino, deve existir pelo menos uma célula computacional do próximo nível mais grosso em todas as direções (à direita, à esquerda, para frente, para trás, para cima e para baixo) que separa este bloco do nível fino de uma célula computacional do segundo próximo nível mais grosso (a menos que este bloco toque a fronteira do domínio computacional, veja a Figura 4.2(f)).

As Figuras 4.3(a) e 4.3(b) são exemplos, em duas dimensões, com dois e três blocos em níveis diferentes que não estão propriamente aninhados. A primeira restrição é violada na Figura 4.3(a) 
e a segunda, é violada na Figura 4.3(b). A Figura 4.3(c) mostra três blocos em níveis diferentes propriamente aninhados. Note que é permitido um bloco de um nível interceptar dois blocos do nível sucessivamente mais grosso.

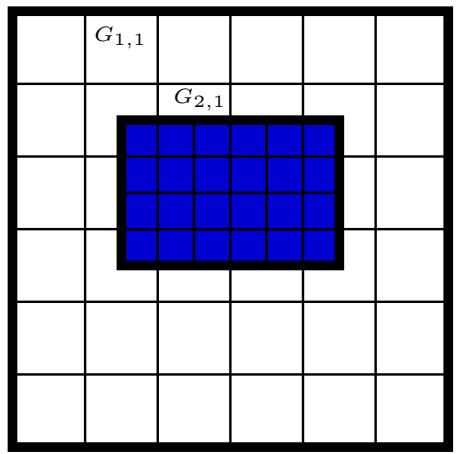

(a) Primeira restrição violada.

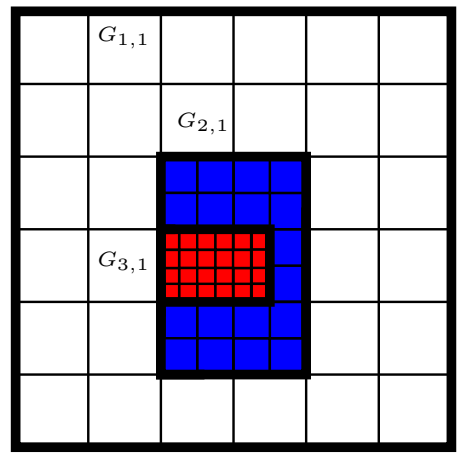

(b) Segunda restrição violada.

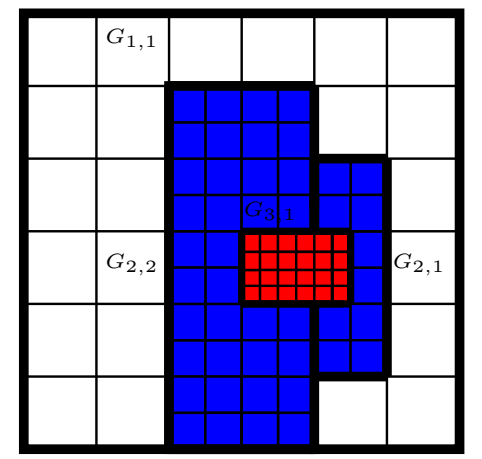

(c) Malha propriamente aninhada.

Figura 4.3: Exemplos de malhas não propriamente aninhadas (a)-(b) e malha propriamente aninhada (c).

As malhas são refinadas no espaço com razão de refinamento $r=2$. Uma importante diferença do trabalho original de Berger e Colella (1989) em relação ao presente trabalho, é que não há refinamento no tamanho do passo de integração, $\Delta t$. Isto significa que a solução numérica, em todos os níveis de refinamento, é determinada com o mesmo tamanho de passo de integração $\Delta t$ dado pelo espaçamento da malha mais fina. Note que isso não significa que o tamanho do passo $\Delta t$ seja contante, veja Seção 3.2. Com isso, garante-se que o erro da discretização espacial e temporal tenham a mesma ordem de aproximação.

\subsection{Geração de uma malha bloco-estruturada}

O número de níveis de refinamento da malha bloco-estruturada é fixo e denotado por $n p l$. Cada nível de refinamento, do mais grosso para o mais fino, é denotado por $l b, l b+1, \ldots, l t$. Aqui abusa-se da linguagem dizendo que o nível base que cobre todo o domínio computacional, $l b$, é o primeiro nível de refinamento. São selecionadas para refinamento células dos níveis $l t-1, l t-2, \ldots, l b$. Quando uma célula de um nível $l$ é selecionada para ser refinada, o resultado deste refinamento são células correspondentes no nível $l+1$.

Dada uma lista de células computacionais de um nível $l$ selecionadas para refinamento, utilizase um método proposto por Berger e Rigoutsos (1991) que agrupa tais células em blocos do nível $l$ (não discretizados) de tal forma que: o volume de todas as células computacionais dadas na lista é coberto. O volume do bloco que não precisa de refinamento e o número de blocos gerados são tão pequenos quanto possíveis. Denota-se por $\epsilon_{\min }$ o parâmetro que ajusta estas duas quantidades por meio da expressão

$$
\epsilon_{\min } \leq \frac{\text { ncells }}{\text { ncellm }}
$$

onde $\epsilon_{\min } \in(0,1)$, ncells é o número de células computacionais selecionadas pelo critério de um bloco no nível $l$ e ncellm é o número total de células computacionais que formam este mesmo bloco no nível $l$. Assim, se $\epsilon_{\min }=0.85$ significa que pelo menos $85 \%$ das células computacionais de um novo bloco de um nível $l$ são células selecionadas pelo critério, veja a Figura 4.4. Este método utiliza-se de técnicas da área de visão computacional e de reconhecimento de padrões. Para mais 
detalhes veja Berger e Rigoutsos (1991). Cada um dos blocos do nível $l$, começando pelos blocos do nível $l t-1$ e decrescendo até $l b$, é discretizado com espaçamento do nível $l+1$. Pelo critério de seleção de células para refinamento, sabe-se que para cada bloco de um nível $l$ existem blocos do nível $l-1$ que o contém. Para garantir a segunda restrição de malhas propriamente aninhadas, os blocos do nível $l-1$ são aumentados com pelo menos duas células computacionais em todas as direções.

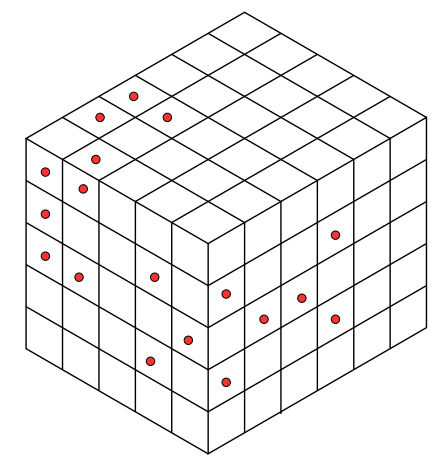

(a) Células selecionadas no nível $l$.

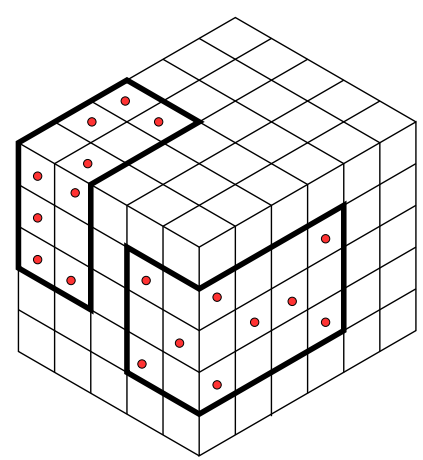

(b) Blocos do nível $l$. A inequação (4.2) está satisfeita.

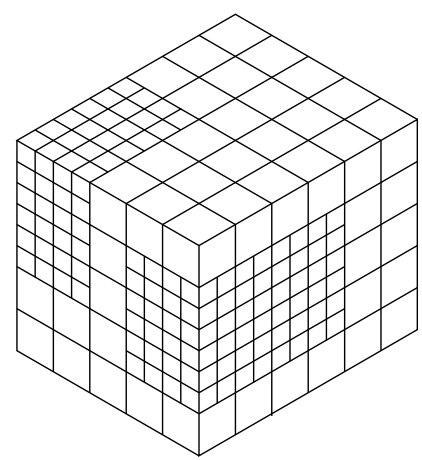

(c) Blocos discretizados gerados no nível $l+1$.

Figura 4.4: Células computacionais selecionadas para a geração nível mais fino.

\subsubsection{Malha inicial do domínio computacional}

A malha bloco-estruturada inicial é gerada por meio de um refinamento estático, de maneira que o refinamento local contenha a região de entrada do escoamento (denominada bocal), veja as Figuras 4.5(a)-4.5(b). Esse refinamento estático continua sendo gerado a cada remalhagem. Esta região precisa de refinamento para que os fenômenos físicos que ocorrem na região de entrada do escoamento sejam bem capturados durante toda a simulação. Assim como as gotículas que são injetadas a cada passo de integração devem estar contidas no nível de refinamento mais fino durante toda a simulação. Detalhes sobre a injeção das gotículas são dados na Seção 6.2.

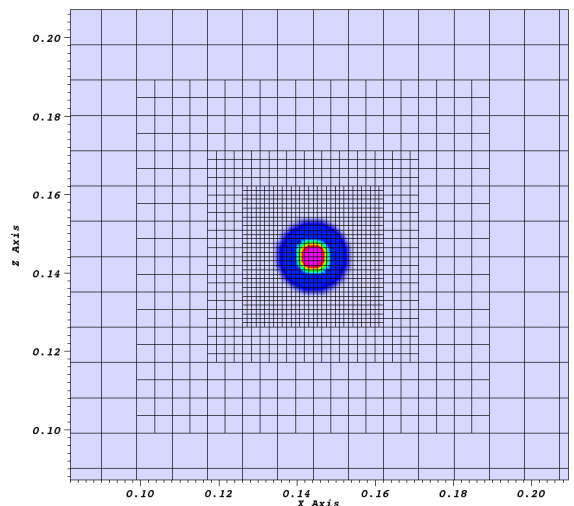

(a) Vista frontal da entrada do escoamento.

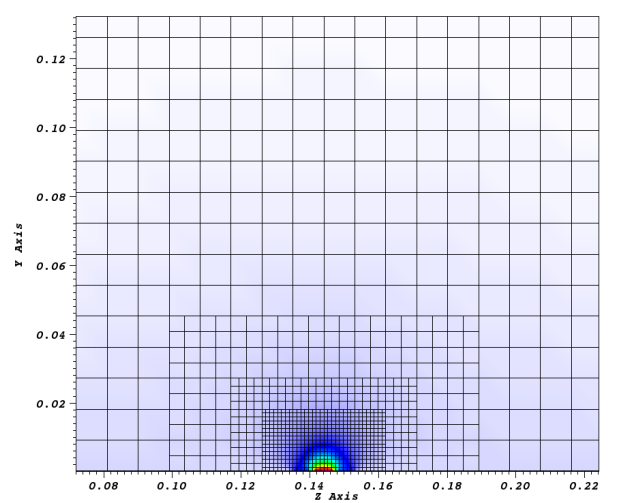

(b) Corte na central na direção $x_{1}$.

Figura 4.5: Zoom de uma malha inicial com quatro níveis de refinamento adaptativo estático e velocidade do escoamento. 


\subsubsection{Seleção de células computacionais para refinamento}

Para cada nível $l$ começando em $l t-1$ e decrescendo até $l b$, células computacionais do nível $l$ são selecionadas (se necessário) para refinamento. Uma célula computacional é selecionada para refinamento se ela satisfaz algum dos critérios descritos abaixo e for uma célula visível. Isto é, não existe um nível mais fino que a cobre. Os critérios utilizados são descritos a seguir.

Se o tensor de tensões viscosas, a vorticidade ou a viscosidade turbulenta, denotadas por $\psi$ satisfazem

$$
\tilde{\psi} \leq \frac{\left|\psi_{i j k}\right|}{\psi_{\max }} \leq 1
$$

onde $\tilde{\psi}$ é um valor no intervalo $[0,1]$. O valor $\tilde{\psi}$ é o valor mínimo da propriedade $\psi$ normalizada, escolhido apropriadamente. Se a temperatura ou a componente da velocidade na direção do escoamento satisfazem

$$
\psi_{i j k} \geq \tilde{\psi}
$$

sendo $\tilde{\psi}$ é um limite inferior da propriedade escolhido apropriadamente. Por exemplo, para a temperatura determinou-se $\tilde{\psi}=$ $800 K$.

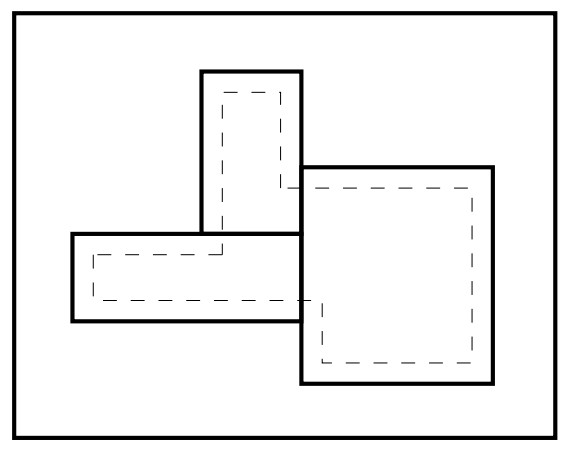

Figura 4.6: Exemplo de região de segurança bidimensional. A região de segurança está contornada por uma linha tracejada.

Os critérios não são utilizados todos ao mesmo tempo em todas as simulações numéricas. A escolha deles depende do problema que está sendo resolvido. Mais detalhes no Capítulo 6.

Quando trata-se de escoamentos com gotículas, mais um critério de refinamento é considerado. Assume-se que todas as gotículas estão contidas no nível de refinamento mais fino. Essa escolha inicial visa garantir que os operadores de interpolação e espalhamento (responsáveis pelo acoplamento entre as fases, veja a Seção 3.3.2) sejam aproximados no nível mais fino de refinamento. Para atender a essa restrição foi definida no nível mais fino da malha bloco-estruturada uma região de segurança (veja um exemplo da região de segurança em duas dimensões na Figura 4.6). Se alguma gotícula estiver fora da região de segurança, a célula computacional correspondente do nível $l t-1$ que contém essa gotícula (mais uma vizinhança) é selecionada para refinamento.

\subsubsection{Localização das gotículas na malha bloco-estruturada}

As gotículas são armazenadas em uma lista encadeada com cabeça a qual possui $N_{p}+1$ células, onde $N_{p}$ é o número de gotículas presentes na simulação. Uma lista encadeada é uma sequência de células, como mostra a Figura 4.7. Cada célula da lista é composta pelo conteúdo e pelo endereço da célula seguinte, para mais detalhes veja Feofiloff (2009).

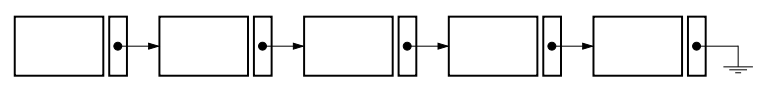

Figura 4.7: Lista encadeada.

Para identificar a célula computacional que contém uma gotícula em uma malha Cartesiana estruturada, basta determinar os índices desta célula computacional por meio da expressão (3.66). 
Na malha bloco-estruturada refinada localmente é necessário identificar a célula computacional que contém a gotícula e o bloco discretizado que contém essa célula computacional. Para fazer esta identificação rapidamente são utilizadas as seguintes estruturas de dados construídas no início da simulação numérica e a cada remalhagem:

- Mapa euleriano: é formado por dois vetores (arrays) com ncell $l_{l t-1}$ posições, onde ncell $l_{l t-1}$ é o número de células computacionais do nível $l t-1$ que estão cobertas pelo nível $l t$. Considere que ambos vetores são indexados por células do nível $l t-1$, isto é, uma posição de qualquer um dos dois vetores é acessada por um identificador de uma célula no nível $l t-1$. A $n$ ésima posição do primeiro vetor armazena um ponteiro para o bloco do nível $l t$ que contém a correspondente célula $n$. A $n$-ésima posição do segundo vetor armazena verdadeiro ou falso, dependendo se a correspondente célula $n$ está ou não na região de segurança.

- Mapa lagrangiano: para cada gotícula $p$ é armazenado um ponteiro para o bloco no nível lt que contém $p$. Considerando todos os ponteiros para todas as gotículas têm-se um mapa lagrangiano.

Os mapas são construídos no início da simulação numérica e a cada remalhagem, pois são nesses momentos que uma nova malha euleriana é construída. A cada passo de integração a localização do bloco discretizado (e assim, da célula computacional) que contém uma gotícula se resume a uma consulta no mapa lagrangiano. A obtenção de tal bloco é importante para a aplicação dos operadores de acoplamento entre as fases dispersa e contínua. Com este bloco, obtém-se rapidamente a célula que contém uma gotícula mais suas células vizinhas. O primeiro mapa euleriano é usado para atualizar o mapa lagrangiano quando uma gotícula sai do bloco que estava. O segundo mapa euleriano é usado para verificar se uma gotícula está ou não na região de segurança, indicando ou não a necessidade de remalhagem.

\subsection{Células computacionais fantasmas}

É importante ressaltar que uma parte essencial da técnica AMR é a existência de células fantasmas, conforme observado por Roma (1996). Os operadores de interpolação e de diferenças finitas descritos na Seção 3.2.4 são aplicados por nível e por bloco discretizado. Os blocos discretizados de um mesmo nível são disjuntos, isto é, dados dois blocos discretizados de um mesmo nível de refinamento, estes não compartilham células computacionais interiores. As células fantasmas são células computacionais auxiliares que contornam cada bloco discretizado (veja um simples exemplo em duas dimensões mostrado pelas Figuras 4.8(a)-4.8(b)). As células fantasmas fornecem condições de fronteiras por meio de interpolações polinomiais de células computacionais de blocos discretizados pertencentes ao mesmo nível de refinamento e que são vizinhos (blocos irmãos, veja como exemplo os blocos $G_{2,1}$ e $G_{2,2}$ na Figura 4.3(c)) e entre blocos discretizados de níveis diferentes. Por exemplo, os valores das propriedades nas células fantasmas pertencentes a um bloco discretizado do nível $l+1$ são obtidos por intermédio da interpolação dos valores entre os blocos discretizados do nível $l$ e $l+1$. 


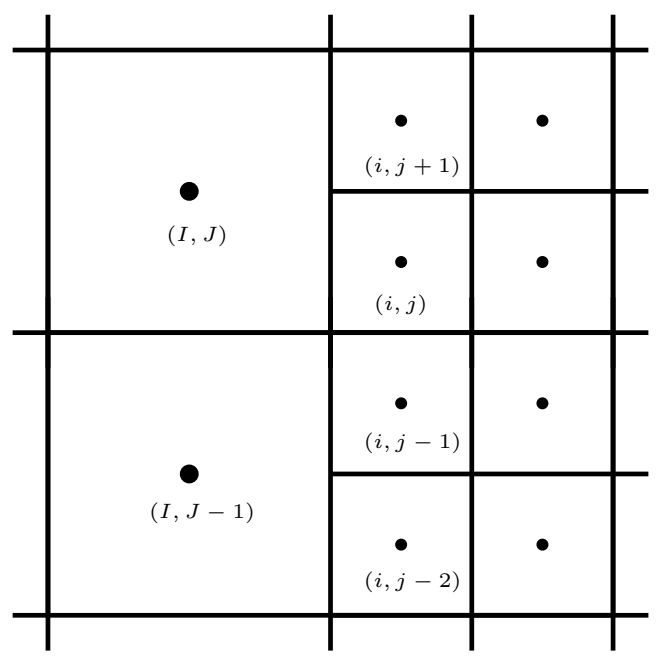

(a) Interface sem células fantasmas.

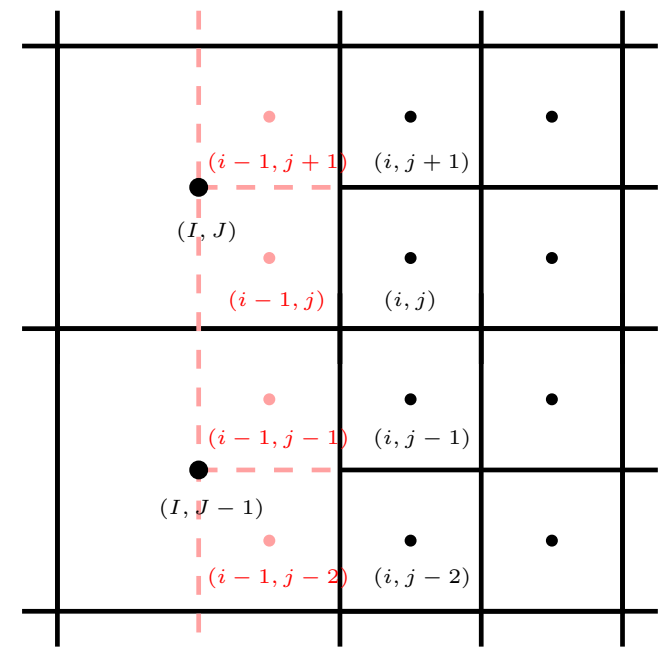

(b) Interface com células fantasmas.

Figura 4.8: Células computacionais fantasmas.

\subsection{Método Multigrid-Multinível}

Uma vez que a discretização espacial na malha bloco-estruturada foi introduzida é necessário, a cada passo de integração temporal, resolver os sistemas lineares resultantes da aproximação. Para resolver estes sistemas lineares é empregado o método multigrid-multinível, para mais detalhes veja Trottenberg et al. (2001). Aqui "multinível" se refere ao fato de que os níveis de refinamento da malha bloco-estruturada (níveis físicos) são também considerados níveis do método multigrid (veja Seção 3.2.5). O método Multigrid-Multinível ciclo V para um problema na malha bloco-estruturada é descrito pelo algoritmo 4.1. Para mais detalhes veja Nós (2007) e Roma (1996).

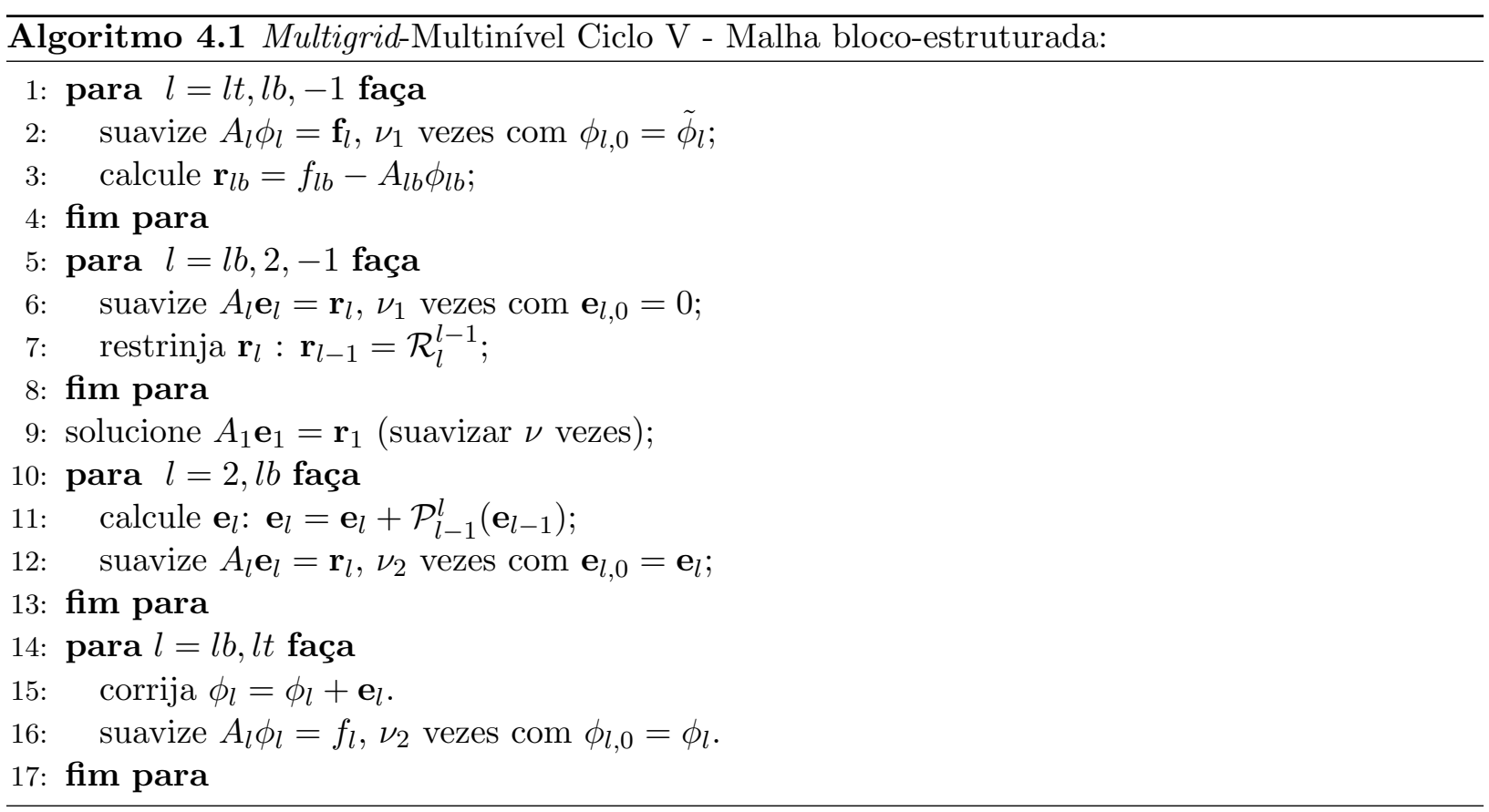

É importante ressaltar que os níveis de refinamento físicos diferem dos níveis multigrid usuais (níveis virtuais) já que eles não cobrem todo o domínio computacional (exceto o nível base). O critério de convergência adotado foi $\mathrm{O}\left(\Delta x^{2}\right)$, sendo $\Delta x=\min \left\{\Delta x_{1, l t}, \Delta x_{2, l t}, \Delta x_{3, l t}\right\}$ o espaçameto 
do nível mais fino $l t$. Os operadores de prolongamento, restrição e suavização são descritos na Seção 3.2.5.

\subsection{Sumário da metodologia numérica na malha bloco-estruturada}

A metodologia numérica com refinamento adaptativo de malhas proposta no presente trabalho visam simular os três modelos matemáticos descritos na Seção 2.6. A diferença entre os algoritmos em uma malha uniforme e em uma malha bloco-estruturada, para os escoamentos sem a fase dispersa, está no procedimento de remalhagem. Para os escoamentos com a fase dispersa aparecem diferenças significativas, por exemplo, o uso dos mapas euleriano e lagrangiano. O Algoritmo 4.2 apresenta, para um passo de integração, uma metodologia numérica com refinamento adaptativo de malhas para o escoamento incompressível (não-reativo) com gotículas inertes. No início de cada passo de integração assume-se que os valores para $\Delta t^{n-1}, \Delta t^{n}, \mathbf{u}^{n-1}, \mathbf{u}^{n}, p_{2}^{n-1}, p_{2}^{n}, \mu^{n-1}, \mu^{n}, \mathbf{X}_{p}^{n}$ e $\mathbf{U}_{p}^{n}$ são conhecidos.

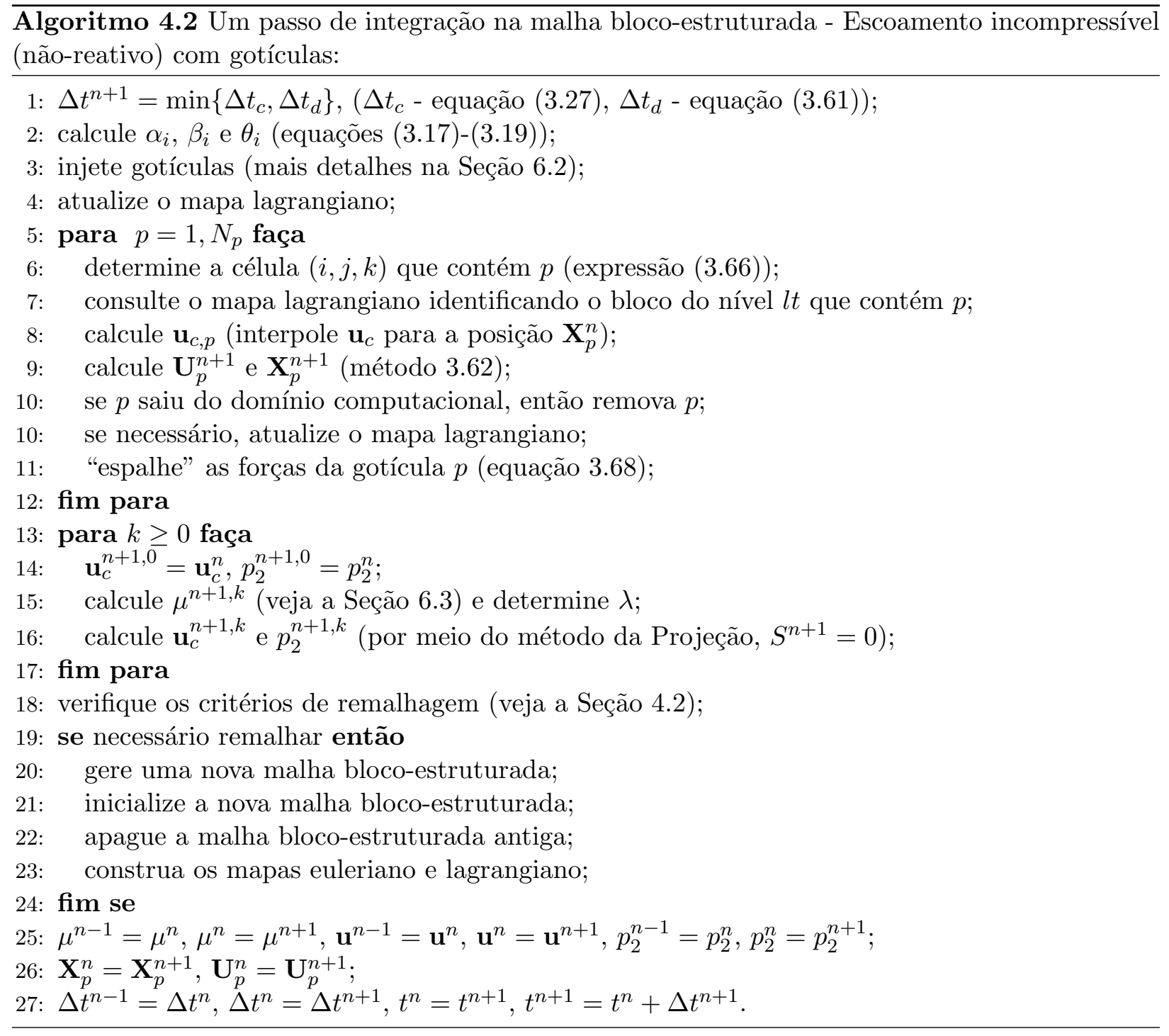

A metodologia numérica dos escoamentos sem a presença de uma fase dispersa em uma malha bloco-estruturada com refinamento adaptativo, descrita nos Algoritmos 3.2 e 3.3, inclui apenas os passos do Algoritmo 4.2 que: verifica os critérios de refinamento, constrói e destrói a malha 
bloco-estruturada, se necessário.

\subsection{Nomenclatura do presente capítulo}

$C_{\text {min }} \quad$ Valor mínimo no critério de refinamento

$G \quad$ Bloco discretizado da malha composta

$l \quad$ Nível de refinamento

$l b \quad$ Nível base de refinamento

lt $\quad$ Nível de refinamento mais fino

ncellm Número de células que formarão o bloco discretizado no nível $l+1$

ncells Número de células computacionais selecionadas para refinamento

$n_{l} \quad$ Número de blocos discretizados do nível $l$

nvl Número de níveis virtuais

npl Número de níveis físicos

$N_{p} \quad$ Número de gotículas

$p_{2} \quad$ Pressão dinâmica $(\mathrm{atm})$

$r \quad$ Razão de refinamento

$t \quad$ Tempo $(s)$

$\mathbf{u} \quad$ Velocidade do escoamento $(\mathrm{m} / \mathrm{s})$

U Velocidade da gotícula $(\mathrm{m} / \mathrm{s})$

\section{Letras Gregas}

$\epsilon_{\min }$ Parâmetro que ajusta o número de células selecionadas para refinamento

$\Delta t \quad$ Tamanho do passo no tempo $(s)$

$\Delta x_{i} \quad$ Espaçamento na direção $i(m)$

$\Omega \quad$ Domínio computacional

$\psi \quad$ Variável escalar

$\widetilde{\psi} \quad$ Valor mínimo da propriedade $\psi$ normalizada

$\nu \quad$ Número de suavizações do método Multigrid-Multinível

Índices

$p$ Gotícula $n$ Tempo

$m$ Blocos discretizados $c$ Fase contínua

\section{Operadores}

$\mathcal{P}$ Prolongamento $\mathcal{R}$ Restrição

\section{Siglas}

AMR Adaptative Mesh Refinement 


\section{Capítulo 5}

\section{Verificação da metodologia numérica}

Neste capítulo, apresentam-se as verificações das implementações dos esquemas numéricos empregados nas discretizações das equações do escoamento da fase contínua e da dinâmica da fase dispersa, descritos nos Capítulos 3 e 4 . Tais verificações numéricas são realizadas empregandose a estratégia de soluções manufaturadas a serem descritas na Seção 5.1. Neste contexto, a Seção 5.2 apresenta a verificação da implementação do esquema numérico empregado para resolver as equações de Navier-Stokes para um escoamento incompressível, a Seção 5.3 a do esquema numérico empregado à equação de advecção-difusão de uma grandeza escalar e, a Seção 5.4, a do esquema numérico completo empregado num escoamento reativo com baixo número de Mach. O Capítulo se encerra com a apresentação da verificação dos esquemas numéricos das equações que modelam a fase dispersa, dos operadores de interpolação e de espalhamento, responsáveis pelo acoplamento entre as fases contínua e dispersa.

\subsection{Verificação numérica por meio de soluções manufaturadas}

A verificação da metodologia numérica foi realizada em um domínio computacional $\Omega=[0,1] \times$ $[0,1] \times[0,1]$, em uma malha composta, bloco-estruturada, com o mesmo espaçamento em todas as direções (isto é, $\Delta x_{1}=\Delta x_{2}=\Delta x_{3}$ ). Esta malha composta possui um nível de refinamento num formato de "L" como mostra a Figura 5.1. As verificações em malhas compostas têm por objetivo confirmar que o uso deste tipo de malha não deteriora a acurácia do método que, quando a solução do problema é bastante suave em todo lugar, deve ser a mesma que se tem em uma malha uniforme, isto é, o uso de uma malha adaptativa no processo de resolução das equações só pode ser benéfico.

As normas $L_{2}$ e $L_{\infty}$, utilizadas para medir os erros das propriedades localizadas no centro da célula computacional, são definidas na malha composta pelas expressões (5.1)-(5.2). Para variáveis vetoriais como a velocidade, localizadas nas faces, tais normas são definidas por (5.3)-(5.4),

$$
\begin{gathered}
\|\psi\|_{2}=\left(\sum_{n}\left|\psi_{n}\right|^{2} \Delta x_{1} \Delta x_{2} \Delta x_{3}\right)^{\frac{1}{2}}, \\
\|\psi\|_{\infty}=\max _{n}\left|\psi_{n}\right|, \\
\left\|u_{i}\right\|_{2}=\left(\sum_{n}\left|u_{i, n}\right|^{2} a_{i, n}\right)^{\frac{1}{2}}, \quad \text { para } i=1,2,3,
\end{gathered}
$$



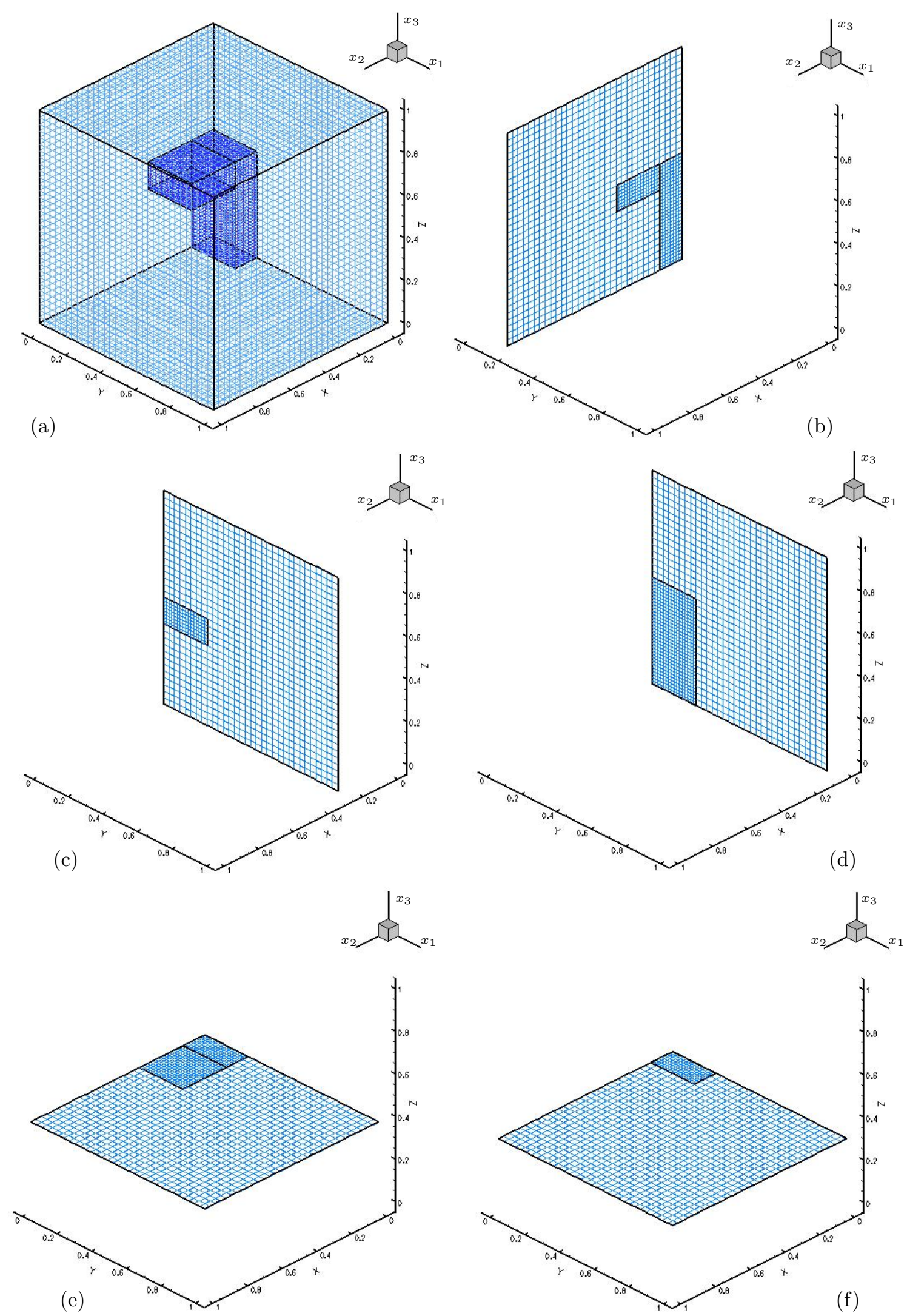

Figura 5.1: Malha composta utilizada na verificação numérica: (a) malha 3D no formato "L", (b) corte no domínio computacional na direção $x_{1}$, (c)-(d) cortes no domínio computacional na direção $x_{2}$ e (e)-(f) cortes no domínio computacional na direção $x_{3}$ (Nós (2007)). 


$$
\left\|u_{i}\right\|_{\infty}=\max _{n}\left|u_{i, n}\right|, \quad \text { para } i=1,2,3,
$$

onde $a_{i, n}$ são os pesos para as normas das componentes do campo de velocidade, necessários na malha composta. Para a componente da velocidade $u_{1}$, na direção $x_{1}$, tem-se

$$
a_{1, n}= \begin{cases}\Delta x_{1} \Delta x_{2} \Delta x_{3} & \text { nas faces } x_{2} x_{3} \text { da malha grossa } \\ \frac{\Delta x_{1} \Delta x_{2} \Delta x_{3}}{r^{2}} & \text { nas faces } x_{2} x_{3} \text { da malha fina } \\ \frac{(2 r+1) \Delta x_{1} \Delta x_{2} \Delta x_{3}}{r^{2}} & \text { nas faces } x_{2} x_{3} \text { da interface da malha grossa-fina }\end{cases}
$$

onde $r$ é a razão de refinamento entre as malhas ( $r=2$, fixado). Os pesos $a_{2, n}$ e $a_{3, n}$ das componentes $u_{2}$ e $u_{3}$ do campo de velocidade são definidos de maneira similar.

Para analisar a convergência dos esquemas numéricos descritos no Capítulo 3, assume-se que a solução numérica pode ser obtida por meio de uma expansão assintótica em potências do espaçamento $h=\min \left(\Delta x_{1}, \Delta x_{2}, \Delta x_{3}\right)$ da malha, conforme descreve Stoer e Bulirsch (2002). Por exemplo, em uma malha uniforme a solução numérica $\psi(\mathbf{x}, \bar{t}, h)$ de uma variável escalar $\psi$ pode ser escrita na forma assintótica como

$$
\psi(\mathbf{x}, \bar{t}, h)=\psi_{e}(\mathbf{x}, \bar{t})+E_{q}(\mathbf{x}, \bar{t}) h^{q}+E_{q+1}(\mathbf{x}, \bar{t}) h^{q+1}+\ldots,
$$

onde $\psi_{e}$ é a solução exata, $E_{i}$ é um coeficiente que não depende de $h, \operatorname{com} i=q, q+1, \ldots, \bar{t}$ é um instante de tempo fixo e $q$ é a ordem de aproximação do método numérico utilizado na aproximação. Desta forma, aproximando a solução em uma malha mais fina ainda, uma com espaçamento $\frac{h}{2}$, obtêm-se

$$
\psi(\mathbf{x}, \bar{t}, h) \approx \psi_{e}(\mathbf{x}, \bar{t})+E_{q}(\mathbf{x}, \bar{t}) h^{q} \quad \text { e } \quad \psi\left(\mathbf{x}, \bar{t}, \frac{h}{2}\right) \approx \psi_{e}(\mathbf{x}, \bar{t})+E_{q}(\mathbf{x}, \bar{t}) \frac{h^{q}}{2^{q}} .
$$

As aproximaçõees na expressão (5.7) implicam na seguinte estimativa para a taxa de convergência do esquema numérico

$$
\frac{\left\|\psi(\mathbf{x}, \bar{t}, h)-\psi_{e}(\mathbf{x}, \bar{t})\right\|}{\left\|\psi\left(\mathbf{x}, \bar{t}, \frac{h}{2}\right)-\psi_{e}(\mathbf{x}, \bar{t})\right\|} \approx 2^{q},
$$

onde $\|\cdot\|$ é uma norma definida por (5.1)-(5.5). Observe que as razões $2^{q}$, obtidas na expressão (5.8), implicam em métodos numéricos de $q$-ésima ordem. A ordem de convergência $q$ é obtida aplicando-se $\log _{2}$ na expressão (5.8). Nas verificações numéricas feitas na malha bloco-estruturada o refinamento é fixo e mantém suas dimensões inalteradas. Nas próximas seções, são apresentados os estudos de convergência numérica por refinamento de malha dos métodos numéricos descritos nos Capítulos 3 e 4 .

\subsection{Escoamento incompressível}

Aqui, apresenta-se a verificação do esquema numérico empregado para resolver as equações de Navier-Stokes que descrevem um escoamento incompressível. Oriundo do trabalho de Nós (2007), a 
implementação desta metodologia numérica evoluiu ao longo do tempo para um código-base denominado "AMR3D", fruto de um trabalho colaborativo com o Laboratório de Dinâmica de Fluidos da Faculdade de Engenharia Mecânica da Universidade Federal de Uberlândia (MFLab/FEMECUFU). A verificação da implementação daquelas equações neste código é o ponto de partida de qualquer desenvolvimento novo. As equações de Navier-Stokes para um escoamento incompressível são dadas por

$$
\begin{aligned}
\rho\left(\frac{\partial u_{i}}{\partial t}+u_{j} \frac{\partial u_{i}}{\partial x_{j}}\right) & =-\frac{\partial p}{\partial x_{i}}+\frac{\partial}{\partial x_{j}}\left[\mu\left(\frac{\partial u_{i}}{\partial x_{j}}+\frac{\partial u_{j}}{\partial x_{i}}\right)\right]+f_{i}, \quad \text { para } i=1,2,3 \\
\frac{\partial u_{j}}{\partial x_{j}} & =0
\end{aligned}
$$

sendo $f_{i}$ o termo forçante que garantirá a igualdade das equações descritas na expressão (5.9) dado por

$$
f_{i}=\rho\left(\frac{\partial u_{i}}{\partial t}+u_{j} \frac{\partial u_{i}}{\partial x_{j}}\right)+\frac{\partial p}{\partial x_{i}}-\frac{\partial}{\partial x_{j}}\left[\mu\left(\frac{\partial u_{i}}{\partial x_{j}}+\frac{\partial u_{j}}{\partial x_{i}}\right)\right] .
$$

Manufatura-se a solução de (5.9)-(5.10) impondo-se as funções

$$
\begin{aligned}
\rho_{e}(\mathbf{x}, t) & =1, \\
\mu_{e}(\mathbf{x}, t) & =\mu_{0}+\mu_{1} \cos ^{2}\left(2 \pi\left(x_{1}+x_{2}+x_{3}\right)+t\right), \\
u_{1, e}(\mathbf{x}, t) & =1+\exp \left(-4 \pi^{2} \lambda t\right)\left[\cos \left(2 \pi\left(x_{2}-t\right)\right)+\sin \left(2 \pi\left(x_{3}-t\right)\right)\right], \\
u_{2, e}(\mathbf{x}, t) & =1+\exp \left(-4 \pi^{2} \lambda t\right)\left[\sin \left(2 \pi\left(x_{1}-t\right)\right)+\cos \left(2 \pi\left(x_{3}-t\right)\right)\right], \\
u_{3, e}(\mathbf{x}, t) & =1+\exp \left(-4 \pi^{2} \lambda t\right)\left[\cos \left(2 \pi\left(x_{1}-t\right)\right)+\sin \left(2 \pi\left(x_{2}-t\right)\right)\right], \\
p_{e}(\mathbf{x}, t) & =\cos \left(2 \pi\left(x_{1}+x_{2}+x_{3}\right)+t\right),
\end{aligned}
$$

com $\mu_{0}=1, \mu_{1}=0.5, \lambda=0.05$ e o campo de velocidade tem divergente zero. As funções utilizadas como solução exata para o campo de velocidade são descritas por Griffith (2005).

Neste teste, foram utilizadas condições de fronteira Dirichlet para as componentes do campo de velocidade e Neumann para a pressão, vindas das soluções exatas. Conforme apresentado na Seção 3.2.1, a escolha das condições de fronteira para a pressão são determinadas pela escolha das condições de fronteira das componentes do campo de velocidade. A Tabela 5.1, apresenta os erros globais e a ordem de aproximação para o campo de velocidade $\mathbf{u}$ e para a pressão $p$ na malha composta apresentada anteriormente, empregando-se o método de Gear extrapolado dado por (3.16). Constata-se segunda ordem para todas as grandezas.

Para a aproximação do campo de velocidade $\mathbf{u}$, espera-se segunda ordem de convergência. Para a pressão espera-se pelo menos primeira ordem de convergência devido a utilização do método da projeção que desacopla a pressão e a velocidade nas equações de Navier-Stokes, conforme descrevem Chorin (1968), Roma (1996) e Nós (2007). Nesta verificação, o tempo final de simulação foi $\bar{t}=0.5$ e foi utilizado o modelo de turbulência LES (Large Eddy Simulation), descrito no Apêndice A. A constante de Smagorinsky utilizada foi $C_{s}=0.18$.

A implementação das equações de Navier-Stokes para um escoamento incompressível (5.9)(5.10) foram verificadas para os diferentes esquemas temporais IMEX descritos na Seção 3.2.1, sendo os erros globais para cada um deles muito similares. Durante o processo de verificação numérica, observou-se que o método de Gear extrapolado gasta mais tempo de processamento. $\mathrm{O}$ 
Tabela 5.1: Erro global e ordem de aproximação para o campo de velocidade e pressão na malha composta.

\begin{tabular}{|c|c|c|c|c|c|c|c|c|}
\hline$N$ & $\left\|u_{1, e}-u_{1}\right\|_{2}$ & $q$ & $\left\|u_{2, e}-u_{2}\right\|_{2}$ & $q$ & $\left\|u_{3, e}-u_{3}\right\|_{2}$ & $q$ & $\left\|p_{e}-p\right\|_{2}$ & $q$ \\
\hline $16^{3}$ & $3.4348 \times 10^{-3}$ & - & $3.4765 \times 10^{-3}$ & - & $3.4310 \times 10^{-3}$ & - & $1.4308 \times 10^{-1}$ & - \\
\hline $32^{3}$ & $8.6952 \times 10^{-4}$ & 1.99 & $8.7751 \times 10^{-4}$ & 1.99 & $8.6033 \times 10^{-4}$ & 1.99 & $4.0890 \times 10^{-2}$ & 1.80 \\
\hline $64^{3}$ & $2.1885 \times 10^{-4}$ & 1.99 & $2.2058 \times 10^{-7}$ & 1.99 & $2.1586 \times 10^{-4}$ & 1.99 & $1.1163 \times 10^{-2}$ & 1.87 \\
\hline $128^{3}$ & $5.4830 \times 10^{-5}$ & 2.00 & $5.5216 \times 10^{-5}$ & 2.00 & $5.4000 \times 10^{-5}$ & 2.00 & $3.0100 \times 10^{-3}$ & 1.94 \\
\hline
\end{tabular}

tempo gasto por passo de integração (normalizado pelo tempo do método de Gear extrapolado) foi, em média, 0.79 para o MCNAB, 0.83 para o CNAB, 0.98 para o CNLF e 1 para Gear extrapolado. Embora o método de Gear extrapolado seja aquele que apresenta o maior tempo computacional por passo de integração, é justo ele aquele que permite maiores passos de integração com garantia de estabilidade sendo portanto, no fim, a escolha mais eficiente.

\subsection{Advecção-difusão de uma grandeza escalar}

Ao AMR3D original, agrega-se a implementação da equação de advecção-difusão de variáveis escalares (por exemplo, fração de mistura),

$$
\frac{\partial(\rho z)}{\partial t}+\frac{\partial\left(\rho u_{j} z\right)}{\partial x_{j}}=\frac{\partial}{\partial x_{j}}\left(D \frac{\partial z}{\partial x_{j}}\right)+f_{z}
$$

onde $f_{z}$ é o termo forçante. Para esta verificação, foram escolhidas funções suaves para $\rho, \mathbf{u}, D$ e $z$ dadas por

$$
\begin{aligned}
z_{e}(\mathbf{x}, t) & =\exp (-t)\left(x_{2}^{3}+x_{3}^{2}\right) \sin \left(\pi x_{1}\right), \\
D_{e}(\mathbf{x}, t) & =\left(x_{1}^{3}+x_{2}^{3}+x_{3}^{3}+1\right) \exp (-t), \\
\rho_{e}(\mathbf{x}, t) & =\left(x_{1}^{2}+x_{2}^{2}+x_{3}^{2}\right) \exp (-t), \\
u_{1, e}(\mathbf{x}, t) & =\sin ^{2}\left(2 \pi\left(x_{1}+x_{2}+x_{3}\right)+t\right), \\
u_{2, e}(\mathbf{x}, t) & =\cos ^{2}\left(2 \pi\left(x_{1}+x_{2}+x_{3}\right)+t\right), \\
u_{3, e}(\mathbf{x}, t) & =\sin \left(2 \pi\left(x_{1}+x_{2}+x_{3}\right)+t\right),
\end{aligned}
$$

Como exemplo, a Tabela 5.2 apresenta os erros e a ordem de aproximação na malha uniforme e na malha composta com um nível de refinamento (Figura 5.1). O tempo final nesta verificação foi $\bar{t}=1$. Note que a segunda ordem de convergência é obtida em ambos os casos.

Tabela 5.2: Erro e ordem de aproximação para a equação do transporte (5.18).

\begin{tabular}{|c|c|c|c|c|c|}
\hline \multicolumn{3}{|c|}{ Malha uniforme } & \multicolumn{3}{c|}{ Malha composta } \\
\hline$N$ & $\left\|z_{e}-z\right\|_{2}$ & $q$ & $N$ & $\left\|z_{e}-z\right\|_{2}$ & $q$ \\
\hline $16^{3}$ & $2.1594 \times 10^{-4}$ & - & $16^{3}$ & $2.1154 \times 10^{-4}$ & - \\
\hline $32^{3}$ & $5.4075 \times 10^{-5}$ & 1.99 & $32^{3}$ & $5.2973 \times 10^{-5}$ & 1.99 \\
\hline $64^{3}$ & $1.3463 \times 10^{-5}$ & 2.00 & $64^{3}$ & $1.3188 \times 10^{-5}$ & 2.00 \\
\hline $128^{3}$ & $3.3660 \times 10^{-6}$ & 2.00 & $128^{3}$ & $3.2950 \times 10^{-6}$ & 2.00 \\
\hline
\end{tabular}




\subsection{Escoamento reativo com baixo número de Mach}

Além da implementação numérica para a advecção-difusão de uma grandeza escalar, é necessária a implementação para a equação da conservação de massa e a generalização do Método da Projeção, originalmente implementada no código AMR3D, para se poder simular escoamentos reativos com baixo número de Mach. Tal generalização é necessária para se poder resolver a correção de pressão que, no contexto de baixo Mach, provém de uma condição de divergência não nula do campo de velocidade. As equações utilizadas para a verificação do modelo matemático completo que descreve a fase contínua, neste caso, são

$$
\begin{aligned}
\frac{\partial \rho}{\partial t}+\frac{\partial\left(\rho u_{j}\right)}{\partial x_{j}} & =\epsilon \frac{\partial^{2} \rho}{\partial x_{j}^{2}}+f_{\rho} \\
\rho\left(\frac{\partial u_{i}}{\partial t}+u_{j} \frac{\partial u_{i}}{\partial x_{j}}\right) & =-\frac{\partial p_{2}}{\partial x_{i}}+\frac{\partial}{\partial x_{j}}\left[\mu\left(\frac{\partial u_{i}}{\partial x_{j}}+\frac{\partial u_{j}}{\partial x_{i}}-\frac{2}{3} \delta_{i j} \frac{\partial u_{k}}{\partial x_{k}}\right)\right]+f_{i}, \quad 1 \leq i \leq 3 \\
\frac{\partial \rho z}{\partial t}+\frac{\partial\left(\rho u_{j} z\right)}{\partial x_{j}} & =\frac{\partial}{\partial x_{j}}\left(\rho D \frac{\partial z}{\partial x_{j}}\right)+f_{z} \\
p_{0} & =\rho z
\end{aligned}
$$

onde $f_{\rho}, f_{i}$ e $f_{z}$ são, respectivamente, os termos forçantes da equação da conservação de massa, da equação da conservação da quantidade de movimento e da equação da advecção-difusão da fração de mistura. O termo de difusão $\rho D$ é dado em função da viscosidade dinâmica $\mu$, já que assume-se $L e=1$, conforme Pember et al. (1998) e Poinsot e Veynante (2005). Assim sendo, tem-se $\rho D=\frac{\mu}{P r}$. A primeira e a última equação, (5.25) e (5.28), combinadas produzem uma restrição não nula para o divergente do campo de velocidade que requer que o Método da Projeção seja extendido, no caso de escoamentos reativos com baixo número de Mach, como descrito na Seção 3.1.1. Neste caso, obtém-se

$$
\frac{\partial u_{j}}{\partial x_{j}}=\frac{1}{z} \frac{D z}{D t}=\frac{1}{p_{0}}\left[\frac{\partial(\rho z)}{\partial t}+\frac{\partial\left(\rho u_{j} z\right)}{\partial x_{j}}\right]
$$

Dependendo do regime do escoamento, há instabilidades numéricas oriundas das discretizações centradas de equações hiperbólicas. Para a equação da conservação de massa (tipo hiperbólico), foi adotada a estratégia de se acrescentar um termo de difusão artificial, $\epsilon=\mathrm{O}(\Delta x)$, para estabilizá-la (veja a Seção 3.2.4) . Assim sendo, o resultado é a equação (5.25), conforme Strikwerda (2004) e Trottenberg et al. (2001). As funções usadas para esta verificação foram

$$
\begin{aligned}
\rho_{e}(\mathbf{x}, t) & =\rho_{0}+\rho_{1} \sin ^{2}\left(2 \pi\left(x_{1}+x_{2}+x_{3}\right)+t\right), \\
z_{e}(\mathbf{x}, t) & =\frac{p_{0}}{\rho_{e}} \\
\mu_{e}(\mathbf{x}, t) & =\mu_{0}+\mu_{1} \cos ^{2}\left(2 \pi\left(x_{1}+x_{2}+x_{3}\right)+t\right), \\
u_{1, e}(\mathbf{x}, t) & =\sin ^{2}\left(2 \pi\left(x_{1}+x_{2}+x_{3}\right)+t\right), \\
u_{2, e}(\mathbf{x}, t) & =\cos ^{2}\left(2 \pi\left(x_{1}+x_{2}+x_{3}\right)+t\right), \\
u_{3, e}(\mathbf{x}, t) & =\sin \left(2 \pi\left(x_{1}+x_{2}+x_{3}\right)+t\right), \\
p_{2, e}(\mathbf{x}, t) & =\cos \left(2 \pi\left(x_{1}+x_{2}+x_{3}\right)+t\right),
\end{aligned}
$$


com $p_{0}=1, \rho_{0}=0.5, \rho_{1}=1, \mu_{0}=1, \mu_{1}=0.5$ e o número de Prandtl, $\operatorname{Pr}=0.75$. Note que o divergente do campo de velocidade é diferente de zero. Na face $x_{1} x_{3}$ em $x_{2}=0$ foram empregadas condições de fronteira Dirichlet para as componentes do campo de velocidade, para o escalar conservado e para a massa específica. Para a pressão $p_{2}$ foi empregada condição de fronteira Neumann. Nas demais faces foram utilizadas condições de fronteira Neumann para as componentes do campo de velocidade, para o escalar conservado e para a massa específica e condição de fronteira Dirichlet para a pressão $p_{2}$. Todas as condições de fronteira são oriundas das soluções manufaturadas. A Tabela 5.3 apresenta os erros e a ordem de aproximação numérica das componentes do campo de velocidade, da pressão dinâmica $p_{2}$, da massa específica $\rho$ e do escalar $z$ na malha composta, obtidas para estas soluções manufaturadas. O tempo final utilizado nesta verificação foi $\bar{t}=0.5$.

Tabela 5.3: Erro e ordem de aproximação do campo de velocidades, da pressão, da densidade e da fração de mistura na malha composta.

\begin{tabular}{|c|c|c|c|c|c|c|}
\hline$N$ & $\left\|u_{1, e}-u_{1}\right\|_{2}$ & $q$ & $\left\|u_{2, e}-u_{2}\right\|_{2}$ & $q$ & $\left\|u_{3, e}-u_{3}\right\|_{2}$ & $q$ \\
\hline $16^{3}$ & $1.7730 \times 10^{-2}$ & - & $1.8726 \times 10^{-2}$ & - & $3.4106 \times 10^{-3}$ & - \\
\hline $32^{3}$ & $4.4185 \times 10^{-3}$ & 2.00 & $4.6506 \times 10^{-3}$ & 2.00 & $9.1857 \times 10^{-4}$ & 1.89 \\
\hline $64^{3}$ & $1.1145 \times 10^{-3}$ & 1.98 & $1.1676 \times 10^{-3}$ & 1.99 & $2.5448 \times 10^{-4}$ & 1.85 \\
\hline $128^{3}$ & $2.8220 \times 10^{-4}$ & 1.98 & $2.9866 \times 10^{-4}$ & 1.96 & $7.0944 \times 10^{-5}$ & 1.84 \\
\hline \hline$N$ & $\left\|p_{2, e}-p_{2}\right\|_{2}$ & $q$ & $\left\|\rho_{e}-\rho\right\|_{2}$ & $q$ & Erro $\left\|z_{e}-z\right\|_{2}$ & $q$ \\
\hline $16^{3}$ & $7.3144 \times 10^{-1}$ & - & $4.0612 \times 10^{-3}$ & - & $3.4434 \times 10^{-3}$ & - \\
\hline $32^{3}$ & $2.0906 \times 10^{-1}$ & 1.80 & $9.8890 \times 10^{-4}$ & 2.03 & $8.3116 \times 10^{-4}$ & 2.05 \\
\hline $64^{3}$ & $5.8046 \times 10^{-2}$ & 1.84 & $2.4180 \times 10^{-4}$ & 2.03 & $2.0215 \times 10^{-4}$ & 2.03 \\
\hline $128^{3}$ & $2.0189 \times 10^{-2}$ & 1.52 & $5.9304 \times 10^{-5}$ & 2.02 & $5.0875 \times 10^{-5}$ & 1.99 \\
\hline
\end{tabular}

\subsection{Fase dispersa}

A fase dispersa, descrita por meio de uma abordagem lagrangiana, é modelada por meio de um sistema de equações diferenciais ordinárias, conforme descrito na Seção 2.4.1. Na verificação numérica, foi utilizada a equação que descreve a posição $\mathbf{X}_{p}$, de cada gotícula $p$, dada por

$$
\begin{aligned}
& \frac{d \mathbf{X}_{p}(t)}{d t}=\mathbf{U}_{p}(t), \\
& \mathbf{X}_{p}(0)=(2.1,0.1,1) .
\end{aligned}
$$

A verificação numérica foi realizada em um domínio computacional $\Omega=[0,3] \times[0,3] \times[0,6]$. As soluções manufaturadas utilizadas foram,

$$
\begin{aligned}
& X_{1, e}(t)=2 \exp (-3 t)+0.1 \\
& X_{2, e}(t)=\frac{100}{1+99 \exp (-0.2 t)}, \\
& X_{3, e}(t)=t+\frac{1}{1-t} .
\end{aligned}
$$

Substituindo as funções descritas na expressão (5.38)-(5.40) nas equações descritas pela ex- 
pressão (5.37), obtêm-se

$$
\begin{aligned}
U_{1, e}(t) & =-3 X_{1, e}(t)+0.3, \\
U_{2, e}(t) & =\frac{198 \exp (0.2 t)}{(99+\exp (0.2 t))^{2}}, \\
U_{3, e}(t) & =1+\left(t-X_{3, e}(t)\right)^{2} .
\end{aligned}
$$

O método de Euler Modificado (3.62) foi utilizado para resolver numericamente o sistema (5.37). A verificação numérica é feita em uma malha uniforme e em uma malha composta. A Tabela 5.4 apresenta os erros e a ordem de aproximação para a verificação numérica do sistema (5.37). Nesta verificação o tempo final de simulação foi $\bar{t}=0.6$. Conforme esperado é obtida segunda ordem de aproximação.

Tabela 5.4: Erro e ordem de aproximação das posições das gotículas.

\begin{tabular}{|c|c|c|c|c|c|c|}
\hline$N$ & $\left\|X_{1, e x}-X_{1}\right\|_{2}$ & $q$ & $\left\|X_{2, e x}-X_{2}\right\|_{2}$ & $q$ & $\left\|X_{3, e x}-X_{3}\right\|$ & $q$ \\
\hline $32^{3}$ & $2.9931 \times 10^{-3}$ & - & $1.1701 \times 10^{-7}$ & - & $4.2890 \times 10^{-2}$ & - \\
\hline $64^{3}$ & $7.4847 \times 10^{-4}$ & 2.00 & $2.9254 \times 10^{-8}$ & 2.00 & $1.0813 \times 10^{-2}$ & 1.99 \\
\hline $128^{3}$ & $1.8714 \times 10^{-4}$ & 2.00 & $7.3152 \times 10^{-9}$ & 2.00 & $2.7155 \times 10^{-3}$ & 1.99 \\
\hline $256^{3}$ & $4.6794 \times 10^{-5}$ & 2.00 & $1.8304 \times 10^{-9}$ & 2.00 & $6.8616 \times 10^{-4}$ & 1.98 \\
\hline $512^{3}$ & $1.1706 \times 10^{-5}$ & 2.00 & $4.5887 \times 10^{-10}$ & 1.99 & $1.7685 \times 10^{-4}$ & 1.96 \\
\hline
\end{tabular}

Para completar a validação da fase dispersa é necessário verificar se a aproximação da função Delta de Dirac, a qual é utilizada para acoplar as fases dispersa e contínua, conforme descrito na Seção 3.3.2 está implementada corretamente. A função utilizada para aproximar a Delta de Dirac é dada pela expressão (3.65). O domínio computacional $\Omega=[0,2] \times[0,2] \times[0,2]$ com um refinamento estático na região $[0.8,2] \times[0.8,2] \times[0.5,1.2]$ é utilizado para a verificação numérica. O campo de velocidade $\mathbf{u}$ é dado pelas funções (5.33)-(5.35). A posição no domínio computacional para ser feita a interpolação do campo de velocidade foi $\mathbf{X}_{p}=(1.2,1.335,0.95)$. A Tabela 5.5 apresenta os resultados da verificação numérica do acoplamento entre as fases no sentido fase contínua para a fase dispersa. Conforme o esperado é obtida segunda ordem nesta aproximação.

Tabela 5.5: Erro e taxa de convergência da interpolação do campo de velocidade do escoamento para a posição $(1.2,1.335,0.95)$.

\begin{tabular}{|c|c|c|c|c|c|c|}
\hline$N$ & $\left\|U_{1, e}-U_{1, i}\right\|_{2}$ & $q$ & $\left\|U_{2, e}-U_{2, i}\right\|_{2}$ & $q$ & $\left\|U_{3, e}-U_{3, i}\right\|$ & $q$ \\
\hline $16^{3}$ & $1.9052 \times 10^{-1}$ & - & $1.9332 \times 10^{-1}$ & - & $1.0514 \times 10^{2}$ & - \\
\hline $32^{3}$ & $5.6170 \times 10^{-2}$ & 1.76 & $5.5428 \times 10^{-2}$ & 1.80 & $2.8237 \times 10^{-3}$ & 1.90 \\
\hline $64^{3}$ & $1.4517 \times 10^{-2}$ & 1.95 & $1.4970 \times 10^{-2}$ & 1.89 & $6.9805 \times 10^{-4}$ & 2.02 \\
\hline $128^{3}$ & $3.7391 \times 10^{-3}$ & 1.96 & $3.5926 \times 10^{-3}$ & 2.06 & $1.8021 \times 10^{-4}$ & 1.95 \\
\hline $256^{3}$ & $9.3327 \times 10^{-4}$ & 2.00 & $9.3916 \times 10^{-4}$ & 1.93 & $4.4822 \times 10^{-5}$ & 2.00 \\
\hline
\end{tabular}




\subsection{Nomenclatura do presente capítulo}

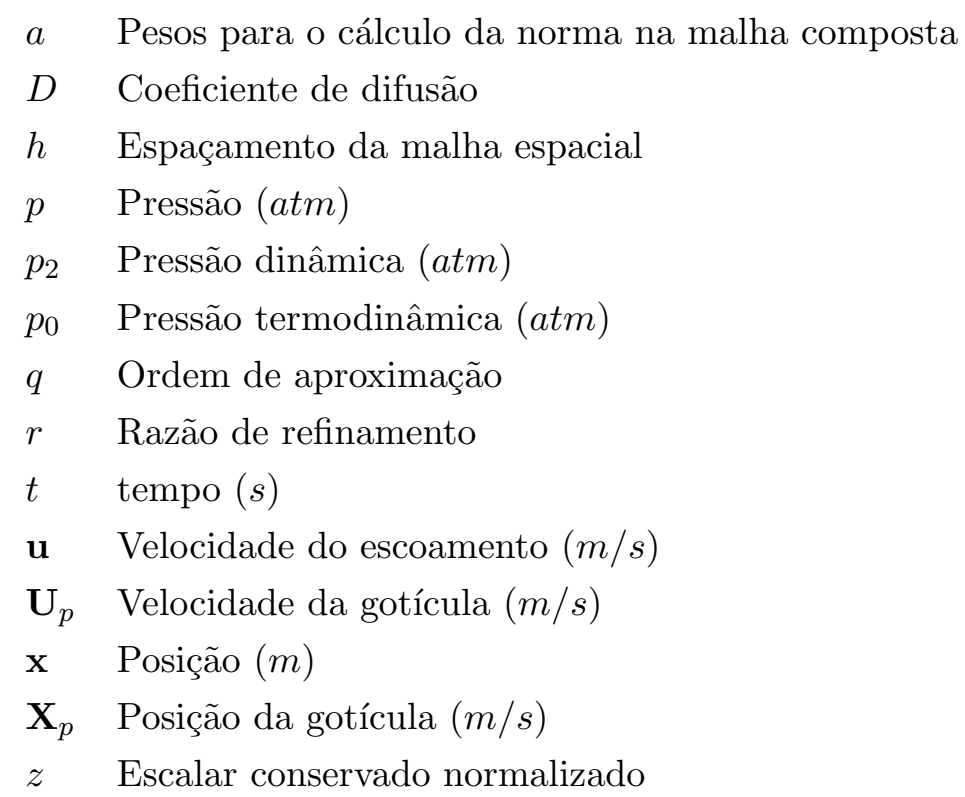

\section{Letras Gregas}

$\Delta x_{i} \quad$ Espaçamento na direção $i(m)$

$\epsilon \quad$ Coeficiente de difusão numérica

$\rho \quad$ Massa específica, densidade $\left(\mathrm{kg} / \mathrm{m}^{3}\right)$

$\Omega \quad$ Domínio computacional

$\psi \quad$ Variável escalar

$\mu \quad$ Viscosidade dinâmica $(\mathrm{kg} / \mathrm{m} \times \mathrm{s})$

\section{Adimensionais}

Le Número de Lewis $\operatorname{Pr} \quad$ Número de Prandtl

Índices

$p$ Gotícula $n$ Número de células computacionais

\section{Operadores}

$\|\cdot\|_{2} \quad$ Norma $L_{2} \quad\|\cdot\|_{\infty} \quad$ Norma do máximo

\section{Siglas}

LES Large-Eddy Simulation

IMEX Esquemas numéricos Implícitos-Explícitos

CNAB Cranck Nicolson Adams-Bashforth

MCNAB Cranck Nicolson Adams-Bashforth Modificado

CNLF Cranck Nicolson Leap-frog 


\section{Capítulo 6}

\section{Resultados numéricos}

O presente capítulo apresenta os resultados da aplicação da metodologia numérica proposta em três situações distintas. Na primeira, abordam-se dois casos de escoamentos incompressíveis (não-reativos): um jato laminar e um jato turbulento. A Seção 6.1 apresenta os resultados obtidos, contendo comparações dos decaimentos da componente axial da velocidade na linha de centro do domínio computacional com decaimentos descritos por Hussein et al. (1994), Turns (1996) e Boersma et al. (1998). Na segunda situação, aplica-se a metodologia numérica proposta a um escoamento incompressível (não-reativo) com gotículas lagrangianas inertes e que não se evaporam. A Seção 6.2 apresenta os resultados preliminares de uma simulação numérica baseada no experimento descrito no trabalho de Stårner e Masri (2003) e nos dados da simulação numérica feita por De et al. (2011). Finalmente, na terceira situação, com a metodologia proposta abordam-se dois escoamentos reativos com baixo número de Mach: um jato laminar e um jato turbulento. Os dados da simulação do jato laminar reativo são baseados no experimento descrito por Smooke et al. (1996). A simulação do jato turbulento reativo é baseada nos dados experimentais da chama de difusão D descrita em Balow e Frank (2007) e simulada numericamente por Pitsch e Steiner (2000) e Kempf et al. (2005), dentre outros. Informações sobre o número de remalhagens, células computacionais utilizadas, estabilidade do esquema temporal e tamanho do passo de integração, dentre outras, são coletadas com o objetivo de se mostrar o desempenho e o potencial da metodologia numérica quando aplicada no contexto aqui considerado.

\subsection{Simulação de escoamentos incompressíveis (não-reativos)}

Para a simulação de jatos incompressíveis (não-reativo) a densidade e a viscosidade dinâmica são constantes, $\rho=1.184 \mathrm{~kg} / \mathrm{m}^{3}$ e $\mu=1.85 \times 10^{-5} \mathrm{~kg} / \mathrm{ms}$. Na Seção 6.1.1, são apresentados os resultados numéricos das simulações de um jato laminar (não-reativo) e na Seção 6.1.2 os resultados da simulação de um jato turbulento não-reativo. As simulações computacionais são feitas empregando-se o modelo matemático dado pelas equações de Navier-Stokes. Dessa forma para um escoamento incompressível têm-se

$$
\begin{aligned}
\rho\left(\frac{\partial u_{i}}{\partial t}+u_{j} \frac{\partial u_{i}}{\partial x_{j}}\right) & =-\frac{\partial p}{\partial x_{i}}+\frac{\partial}{\partial x_{j}}\left[\mu\left(\frac{\partial u_{i}}{\partial x_{j}}+\frac{\partial u_{j}}{\partial x_{i}}\right)\right], \text { para } i=1,2,3 \\
\frac{\partial u_{j}}{\partial x_{j}} & =0
\end{aligned}
$$


onde $\rho$ é a massa específica, $u_{i}$ é a componente do campo de velocidade em cada uma das direções, $p$ é a pressão e $\mu$ é a viscosidade dinâmica. Para o caso turbulento utilizou-se o modelo de turbulência LES (veja o Apêndice A), após a filtragem das equações (6.1)-(6.2) o termo $\mu$ representa a viscosidade dinâmica mais a viscosidade turbulenta $\mu_{t}=\left(C_{s} \Delta\right)^{2}\left|\mathcal{S}_{i j}\right|$, onde $\Delta=\left(\Delta x_{1} \Delta x_{2} \Delta x_{3}\right)^{\frac{1}{3}}$ é o tamanho do filtro, $\left|\mathcal{S}_{i j}\right|$ é o módulo da taxa de deformação dada por

$$
\left|\mathcal{S}_{i j}\right|=\sqrt{2 S_{i j} S_{i j}}, \quad \text { com } S_{i j}=\frac{1}{2}\left(\frac{\partial u_{i}}{\partial x_{j}}+\frac{\partial u_{j}}{\partial x_{i}}\right)
$$

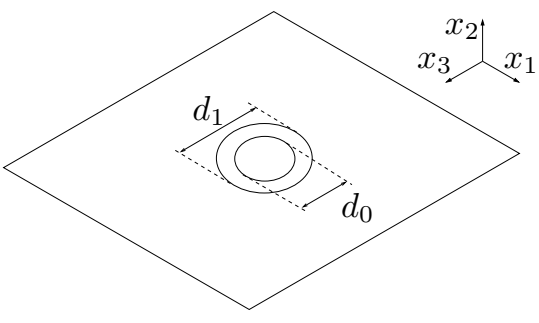

e $C_{s}$ é a constante de Smagorinsky, $0.1 \leq C_{s} \leq 0.18$ (veja Pope (2000)). Para os resultados que se seguem, foi utilizada $C_{s}=0.15$.

A Figura 6.1 mostra um esboço da entrada do domínio computacional composta por dois discos concêntricos, o menor denominado bocal com diâmetro $d_{0}$ e o segundo, denominado piloto que serve para estabilizar a velocidade (no caso turbulento) cujo diâmetro é $d_{1}$. Na entrada do domínio computacional, são usadas condição de fronteira Dirichlet para as componentes do campo de velocidade e condição de fronteira Neumann nula para a correção da pressão. Nas demais faces do domínio computacional, são usadas as condições de fronteira Neumann nulas para as componentes do campo de velocidade e a condição de fronteira Dirichlet para a correção da pressão.

\subsubsection{Escoamento incompressível (não-reativo) laminar}

Os dados da simulação do jato laminar (não-reativo) foram baseados nos dados da simulação do jato laminar reativo descritos por Pember et al. (1998). O diâmetro do bocal do jato laminar é $d_{0}=4 \times 10^{-3} \mathrm{~m}$. As dimensões do domínio computacional são $0.16 \mathrm{~m} \times 0.16 \mathrm{~m} \times 0.16 \mathrm{~m}$. A velocidade na entrada do domínio é $u_{2, d_{0}}=0.25 \mathrm{~m} / \mathrm{s}$ no bocal, $u_{2}=2 \times 10^{-3} \mathrm{~m} / \mathrm{s}$ na região piloto e no restante da entrada (veja a Figura 6.1). O número de Reynolds do jato laminar baseado na medida do diâmetro do bocal e na velocidade $u_{2, d_{0}}$ é $R e \approx 65$.

A simulação foi realizada em uma malha cartesiana bloco-estruturada tridimensional adaptativa, composta por uma malha base mais três níveis de refinamento (quatro níveis no total). A malha base possui $32 \times 32 \times 32$ células computacionais. O nível de refinamento mais fino possui espaçamento $6.25 \times 10^{-4} \mathrm{~m}$. Isso garante que a região de saída do jato contenha pelo menos 6 células computacionais do nível mais fino.

A seleção das células computacionais que geram um nível de refinamento é descrita na Seção 4.2. O critério de refinamento utilizado nesta simulação foi baseado na norma do tensor de tensões viscosas, por meio da expressão (4.3) e no valor da componente da velocidade na direção do escoamento por meio da expressão (4.4). O tamanho do passo de integração é determinado pela condição CFL (4.1) sendo $\Delta t=\mathrm{O}\left(\Delta x^{0.988}\right)$.

A Figura 6.3 mostra a componente da velocidade na direção do escoamento em um plano de corte central do domínio computacional com a malha bloco-estruturada de quatro níveis de refinamento. As Figuras 6.2(a)- 6.2(f) mostram, em um plano de corte no centro do domínio computacional, a evolução da componente da velocidade na direção do escoamento do jato laminar 
(não-reativo).

Segundo Kuo (2005), Turns (1996) e Boersma et al. (1998), o decaimento da componente da velocidade na direção do escoamento para um jato laminar na linha de centro do domínio computacional é descrito pela expressão

$$
\frac{u_{2}^{*}}{u_{d_{0}}}=0.375 R e_{r}\left(\frac{r_{0}}{x_{2}}\right)
$$

onde $u_{2}^{*}$ é a velocidade do escoamento na linha de centro na direção do escoamento, $u_{d_{0}}$ é a velocidade do escoamento na saída do bocal, $r_{0}$ é a medida do raio do bocal e $R e_{r}$ é o número de Reynolds baseado na medida de $r_{0}$, neste caso, $R e_{r} \approx 37.5$. A Figura 6.4 mostra o decaimento da componente da velocidade na direção do escoamento obtido nesta simulação e o decaimento descrito pela expressão (6.4). É importante ressaltar, conforme Turns (1996), que a comparação do decaimento é válida a partir $x_{2}^{*}=\frac{x_{2}}{r_{0}} \gtrsim 0.375 R e_{r}$, o qual, neste caso, é equivalente a $\frac{x_{2}}{d_{0}} \approx 10$. É necessário investigar a diferença entre os decaimentos na Figura 6.4 utilizando malhas com mais níveis de refinamento, aumentando o número de células computacionais no bocal a fim de descrever melhor a velocidade.

Para comparar o benefício do uso da malha composta (bloco-estruturada) versus o uso da malha uniforme, o mesmo problema foi simulado em uma malha uniforme equivalente ao nível mais fino da simulação do escoamento

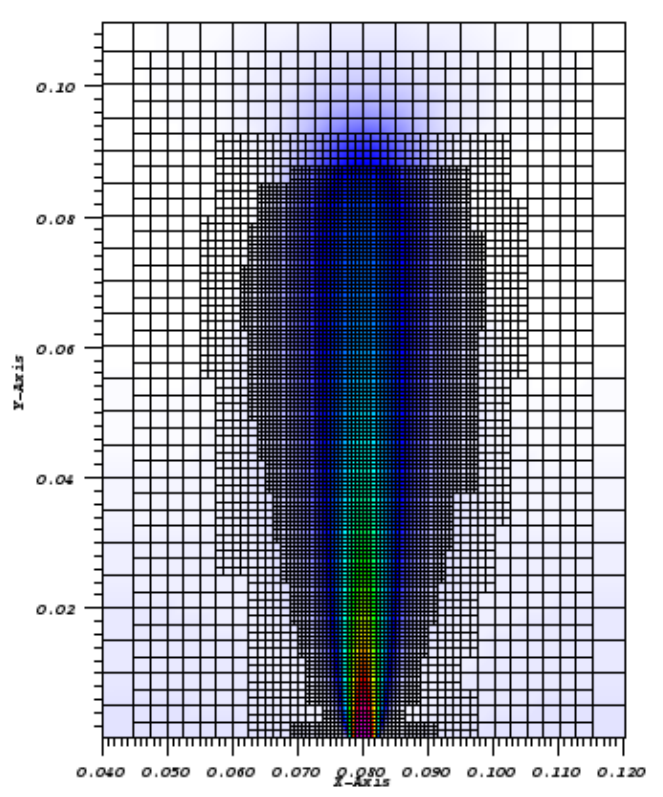
laminar, uma malha uniforme dada por $256 \times 256 \times 256$

Figura 6.3: Zoom da velocidade do escoamento em um corte central e malha blocoestruturada .

células computacionais. A razão entre o tempo computacional gasto na malha uniforme e o tempo gasto na malha bloco-estruturada adaptativa (AMR) indica que no início da simulação numérica a malha AMR chegou a ser 146 vezes mais rápida que a malha uniforme e nos instantes de tempo finais, chegou a ser 16 vezes mais rápida. A simulação numérica do escoamento laminar realizou aproximadamente 50 remalhagens ao longo dos quase $30 \times 10^{3}$ passos de integração. A Figura 6.5 mostra a razão entre os tempos gastos em uma malha uniforme (TMU) e em uma malha composta (TMC) bloco-estruturada. A partir do passo de integração $2 \times 10^{3}$ o consumo de tempo por passo de integração é mantido aproximadamente constante.

\subsubsection{Escoamento incompressível (não-reativo) turbulento}

A simulação do jato turbulento incompressível (não-reativo) empregou os dados da velocidade e domínio físico da simulação da chama de difusão, descritos nos trabalhos de Balow e Frank (2007), Kempf et al. (2005) e Pitsch e Steiner (2000). O diâmetro do bocal é $d_{0}=7.2 \times 10^{-3} \mathrm{~m}$ e o diâmetro da região piloto é $d_{1}=1.8 \times 10^{-2} \mathrm{~m}$. As dimensões do domínio computacional nesta simulação são $40 d_{0} \times 80 d_{0} \times 40 d_{0}$, aproximadamente $0.288 m \times 0.576 m \times 0.288 m$.

As componentes da velocidade na entrada do domínio são dadas por $u_{1, \text { in }}=u_{3, \text { in }}=0 \mathrm{e}$ 


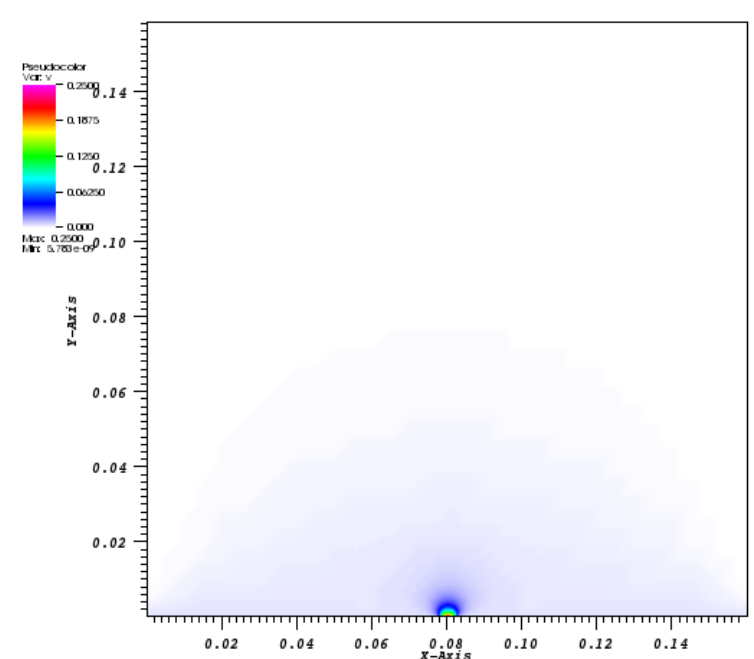

(a)

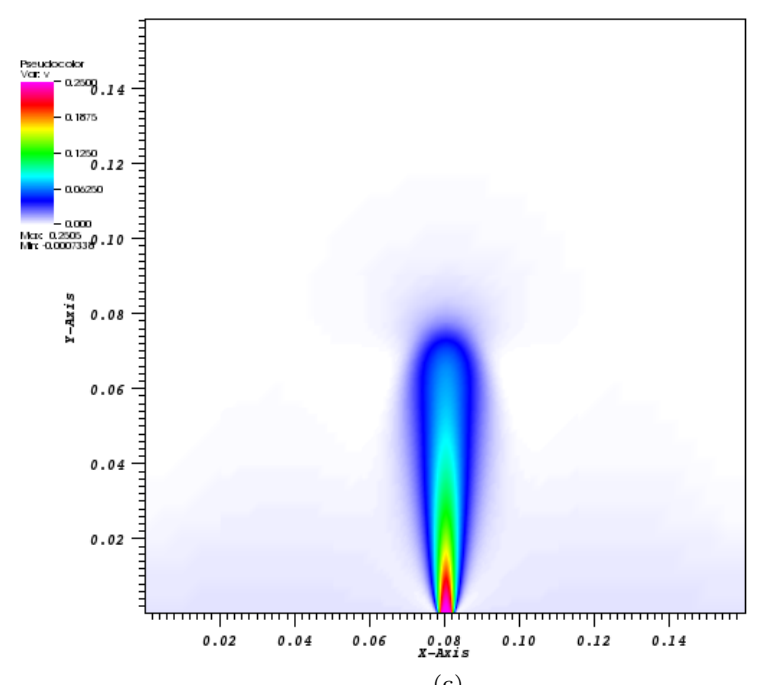

(c)

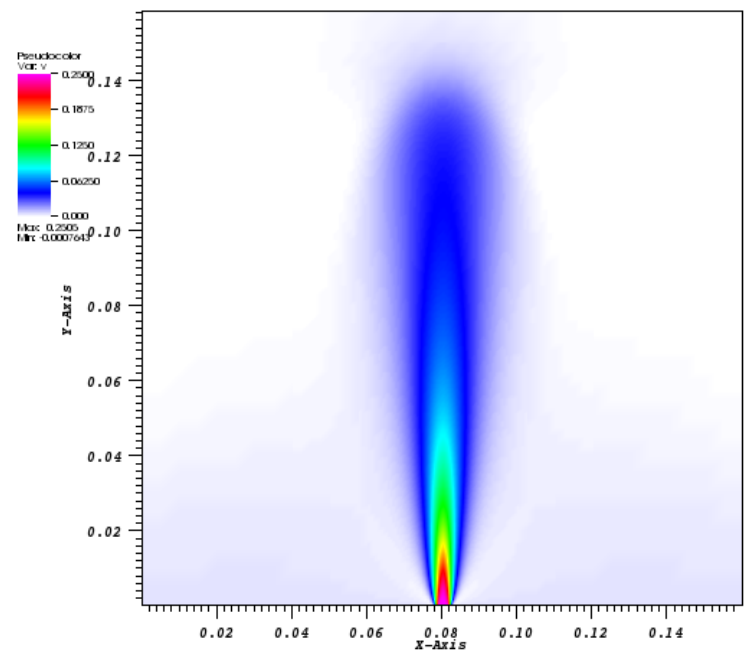

(e)

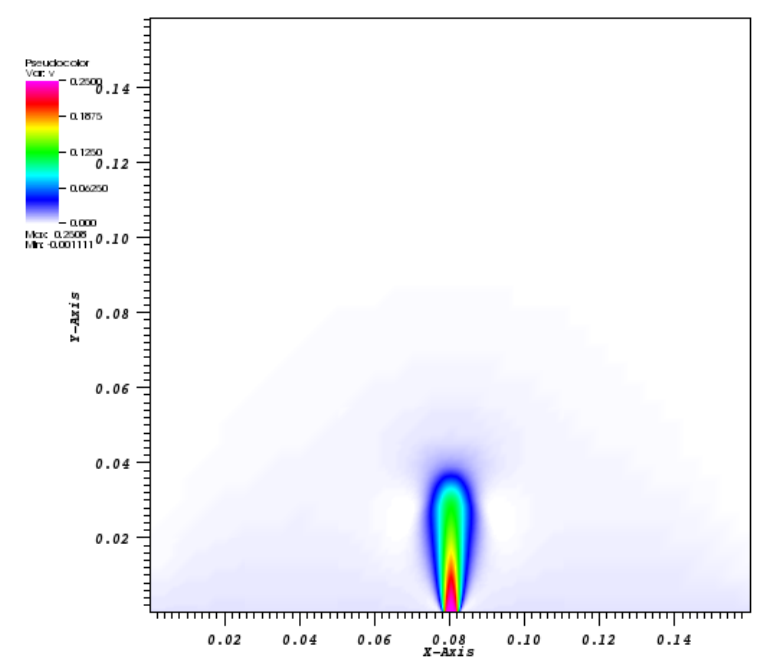

(b)

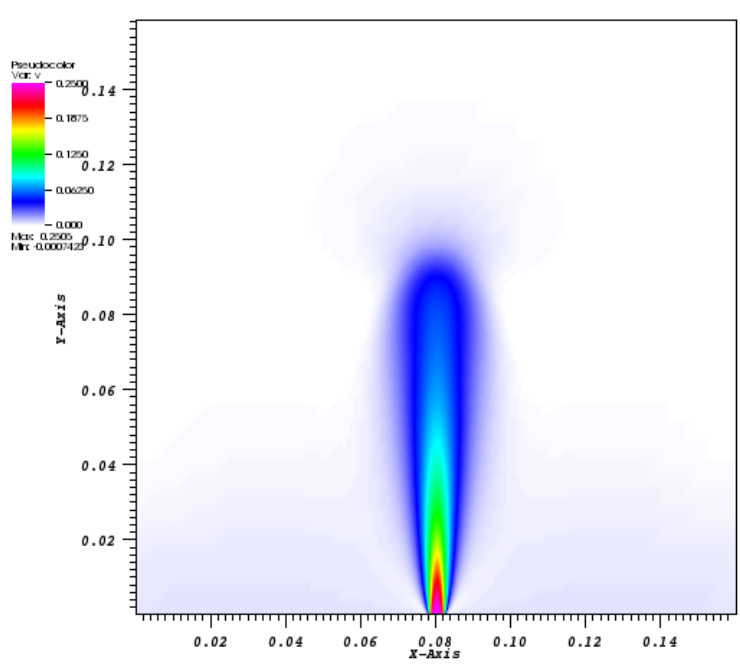

(d)

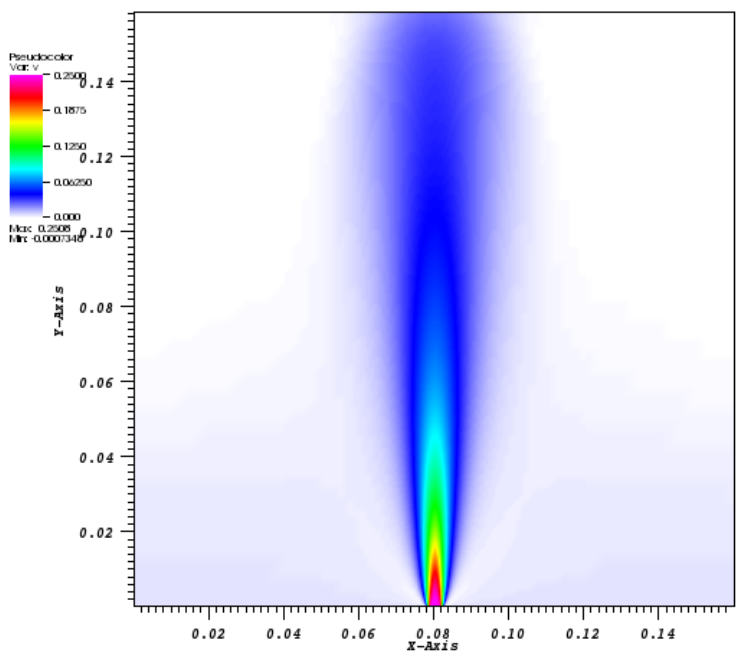

(f)

Figura 6.2: Escoamento incompressível (não-reativo) laminar: componente da velocidade na direção do escoamento em um plano de corte no domínio computacional em $x_{3}=8 \times 10^{-2} \mathrm{~m}$ em diferentes instantes de tempo. 


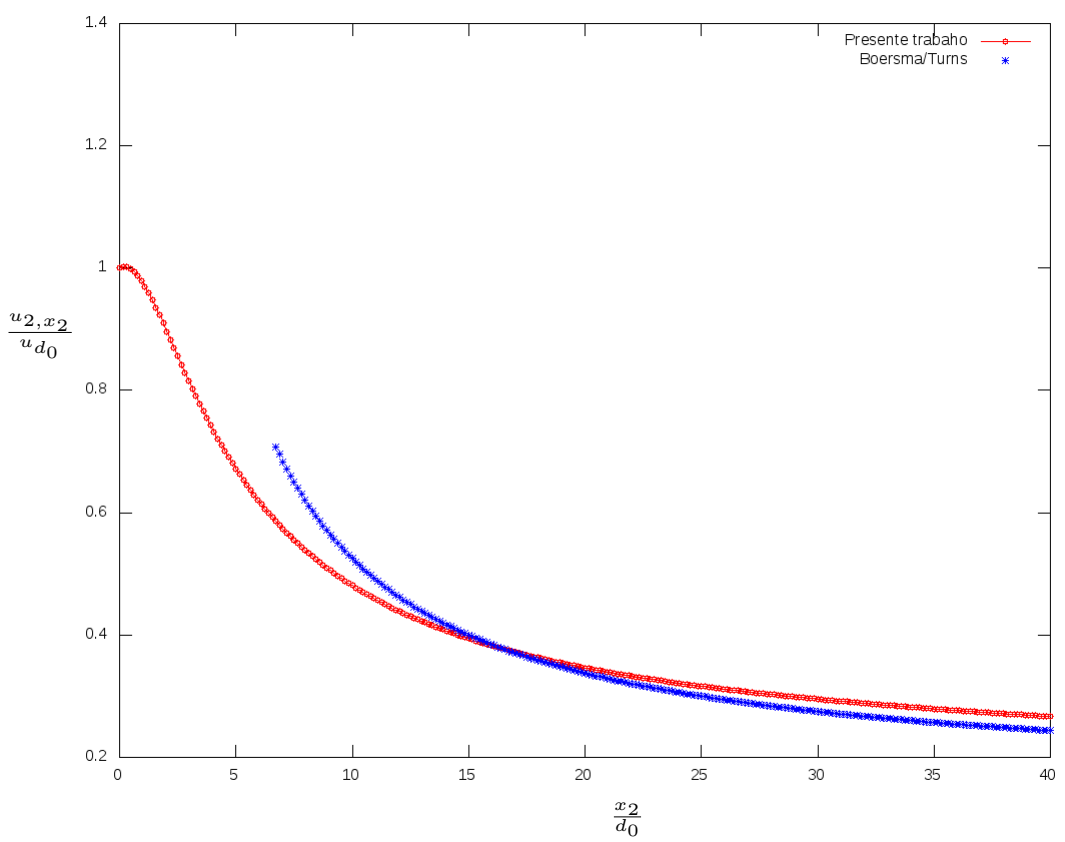

Figura 6.4: Decaimento da velocidade na direção do escoamento na linha de centro do domínio computacional.

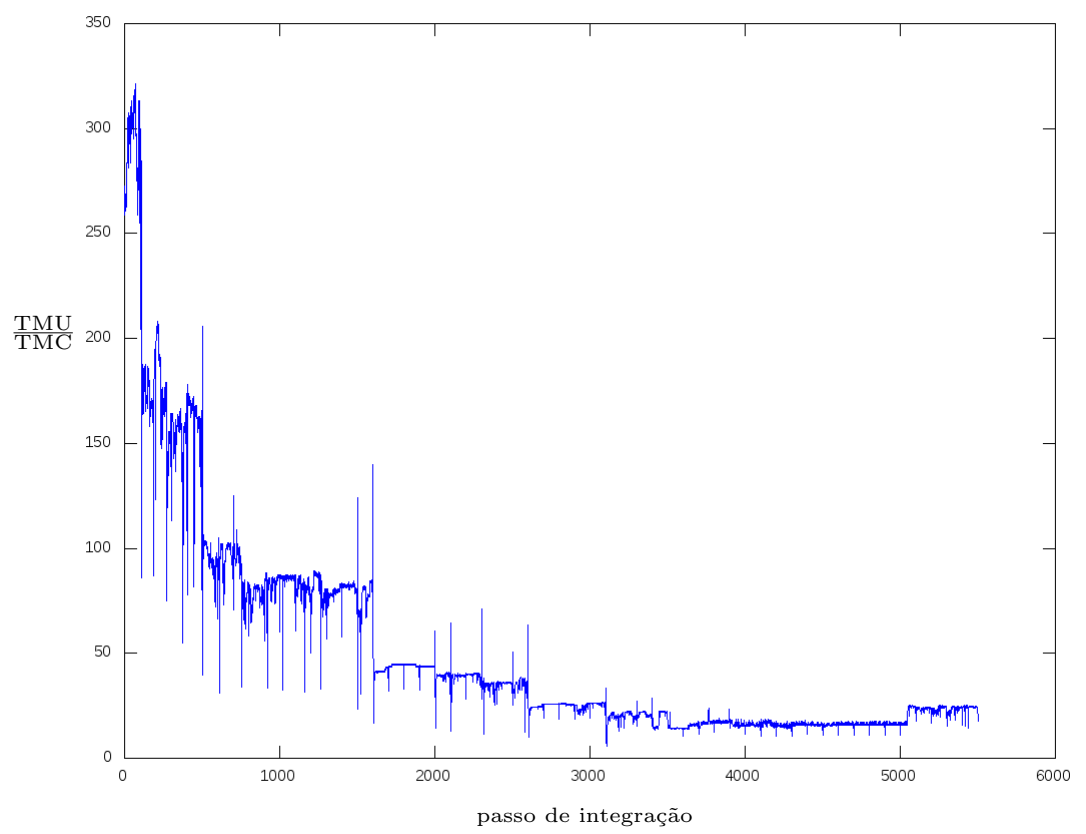

Figura 6.5: Razão entre os tempos gastos em uma malha uniforme (TMU) e em uma malha composta bloco-estruturada (TMC).

$u_{2, i n}=u_{d_{0}}+u_{d_{1}}, \mathrm{com}$

$$
\begin{aligned}
& u_{d_{1}}=\frac{v_{1}+v_{0}}{2}-\left(\frac{v_{1}-v_{0}}{2}\right) \tanh \left[25\left(\frac{r_{j}}{r_{1}}-\frac{r_{1}}{r_{j}}\right)\right] \\
& u_{d_{0}}=\frac{v_{2}+v_{0}}{2}-\left(\frac{v_{2}-v_{0}}{2}\right) \tanh \left[25\left(\frac{r_{j}}{r_{0}}-\frac{r_{0}}{r_{j}}\right)\right],
\end{aligned}
$$


onde $r_{j}=\sqrt{\left(x_{3}-0.5 \cdot b_{3}\right)^{2}+\left(x_{1}-0.5 \cdot b_{1}\right)^{2}}, v_{0}=0.9 \mathrm{~m} / \mathrm{s}, v_{1}=11.4 \mathrm{~m} / \mathrm{s}$ e a velocidade do jato principal é $v_{2}=49.6 \mathrm{~m} / \mathrm{s}, r_{1}$ e $r_{0}$ são, respectivamente, os raios da região piloto e do bocal, $b_{1}$ e $b_{3}$ são os tamanhos do domínio nas direções $x_{1}$ e $x_{3}$, respectivamente.

Além disso, foi acrescentada uma perturbação (ruído branco) na componente $u_{2}$ do campo de velocidade na direção do escoamento para simular a turbulência na entrada do domínio computacional conforme descreve Boersma et al. (1998), dada por $0.01 \times$ ran $\times u_{2}$, onde ran é um número aleatório gerado uniformemente entre 0 e 1.

O número de Reynolds baseado no diâmetro do bocal é $R e=2.24 \times 10^{4}$. A simulação foi realizada em uma malha bloco-estruturada composta por uma malha base mais três níveis de refinamento adaptativo (quatro níveis no total). A malha base possui $32 \times 64 \times 32$ células computacionais. A Figura 6.6 mostra uma malha blocoestruturada utilizada nesta simulação. O nível mais fino possui espaçamento $1.125 \times 10^{-3} \mathrm{~m}$, o qual garante que

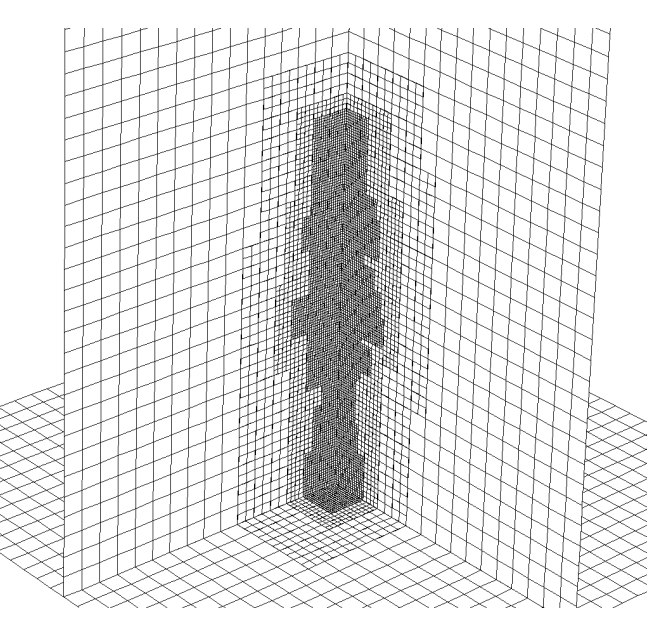

Figura 6.6: Malha tridimensional com refinamento adaptativo. o diâmetro do bocal contenha pelo menos 6 células computacionais. O que pode não ser suficiente para representar a física do problema, veja comentários na Seção 6.4. O critério de refinamento usado nesta simulação foi baseado na norma do tensor de tensões viscosas (o qual se mostrou equivalente ao critério baseado na vorticidade) e na norma da viscosidade turbulenta com $85 \%$ de eficiência (isto é, a razão entre o número de células que precisam ser refinadas sobre o número de células que formarão um bloco discretizado, veja a Seção 4.2). A marcação das células computacionais que necessitam de refinamento é descrita na Seção 4.2. Para esta simulação numérica, o tamanho do passo de integração, determinado pela condição CFL (4.1) foi $\Delta t=\mathrm{O}\left(\Delta x^{1.65}\right)$. As Figuras 6.7(a)-(f) mostram, em um corte central do domínio computacional, a evolução da componente da velocidade na direção do escoamento. A Figura 6.8 mostra a componente da velocidade na direção do escoamento e os detalhes da malha bloco-estruturada gerada, com quatro níveis de refinamento. As Figuras 6.9-6.10 mostram, em cortes transversais do domínio computacional em 15\%, 25\%, 50\% e 75\% na direção do escoamento, a componente da velocidade na direção do escoamento junto com a malha bloco-estruturada com quatro níveis de refinamento em um mesmo instante de tempo. Os cortes são, respectivamente, em $x_{2}=0.0864 \mathrm{~m}$ nas Figuras 6.9(a)-(b), $x_{2}=0.144 m$ nas Figuras 6.10(a)-(b), $x_{2}=0.288 m$ nas Figuras 6.10(c)(d) e $x_{2}=0.432 m$ nas Figuras 6.10(e)-(f). As Figuras 6.11(a)-6.11(b) mostram detalhes da malha composta com refinamento adaptativo. A Figura 6.11(a) mostra, em um corte no centro do domínio computacional, a componente da velocidade na direção do escoamento junto com o refinamento adaptativo. A Figura 6.11(b) mostra os blocos discretizados que compõem a malha bloco-estruturada com refinamento adaptativo. Os contornos identificam os diferentes blocos discretizados de cada nível de refinamento. Note que a união de blocos discretizados com mesmo espaçamento formam um nível de refinamento.

O decaimento médio da velocidade de jatos turbulentos incompressíveis na linha de centro é 


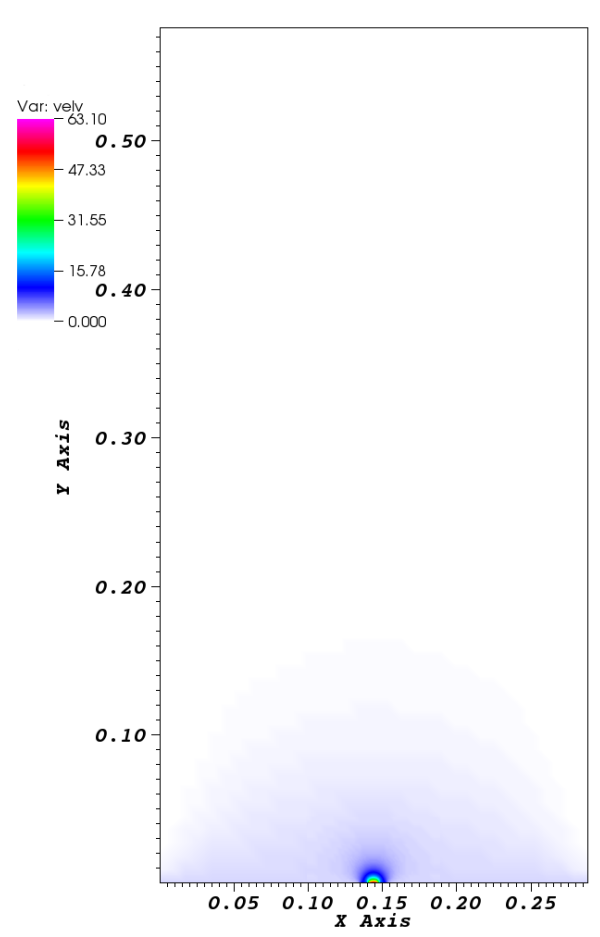

(a)

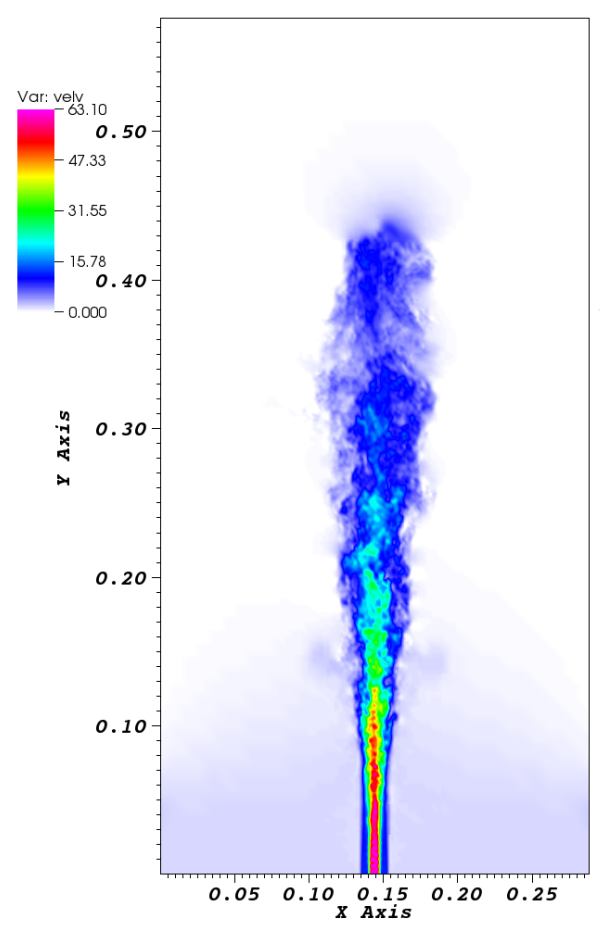

(d)

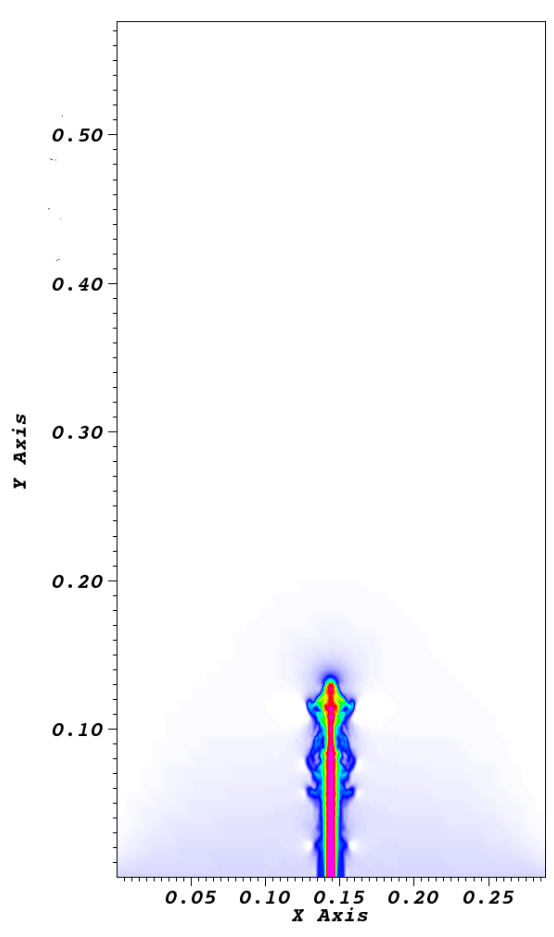

(b)

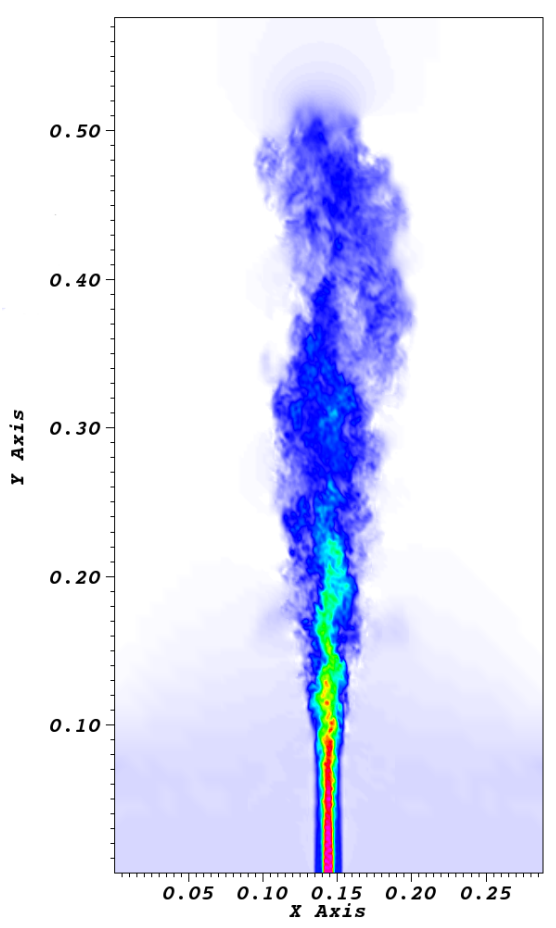

(e)

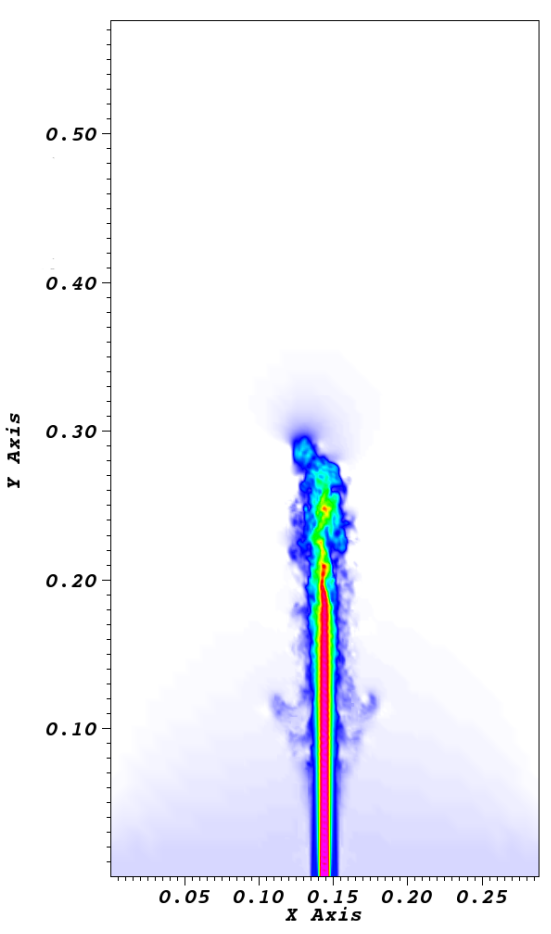

(c)

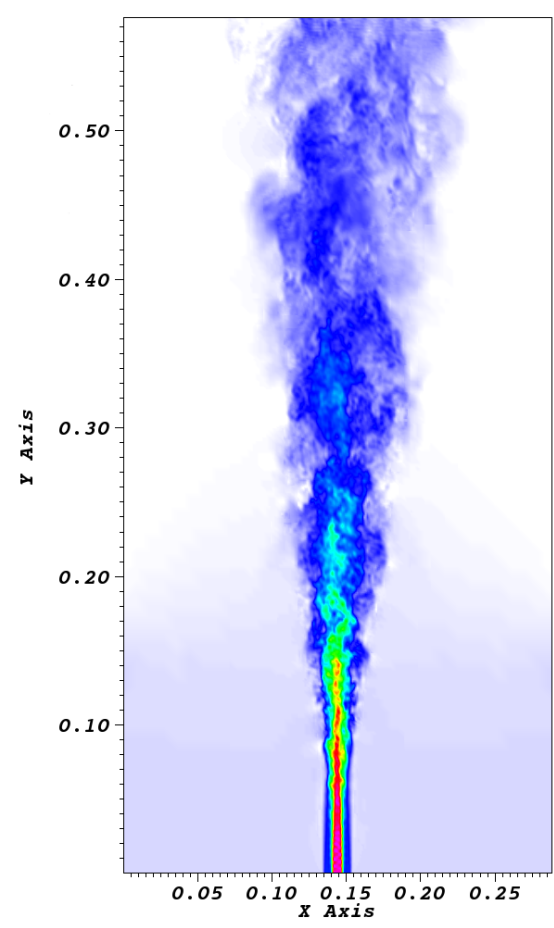

(f)

Figura 6.7: Escoamento incompressível (não-reativo) turbulento: componente da velocidade na direção do escoamento em um plano de corte no centro do domínio computacional em $x_{3}=1.44 \times 10^{-1} \mathrm{~m}$.

descrito pela expressão

$$
\frac{u_{2}^{*}}{u_{d_{0}}}=\frac{B_{u} d_{0}}{\left(x_{2}-x_{0}\right)}
$$

onde $B_{u}=5.8$ e $x_{0}=4 d_{0}$, conforme Hussein et al. (1994) e Boersma et al. (1998). Boersma et al. (1998) descreve que o perfil do decaimento da velocidade depende do diâmetro do bocal e da velocidade inicial do jato. A Figura 6.12 mostra o decaimento obtido nesta simulação e o decaimento 

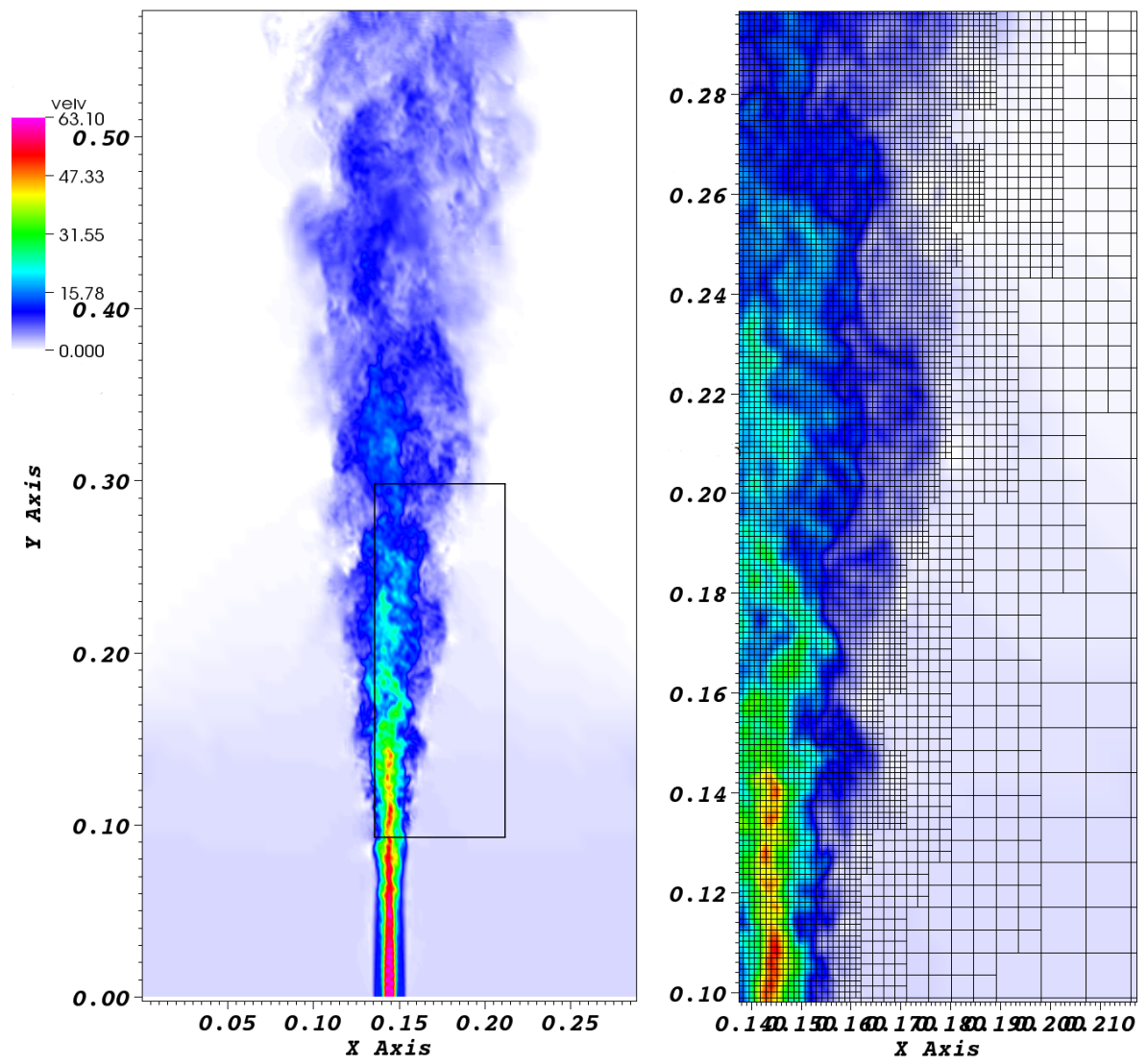

Figura 6.8: Velocidade do jato na direção do escoamento com a malha bloco-estruturada em um plano de corte central.
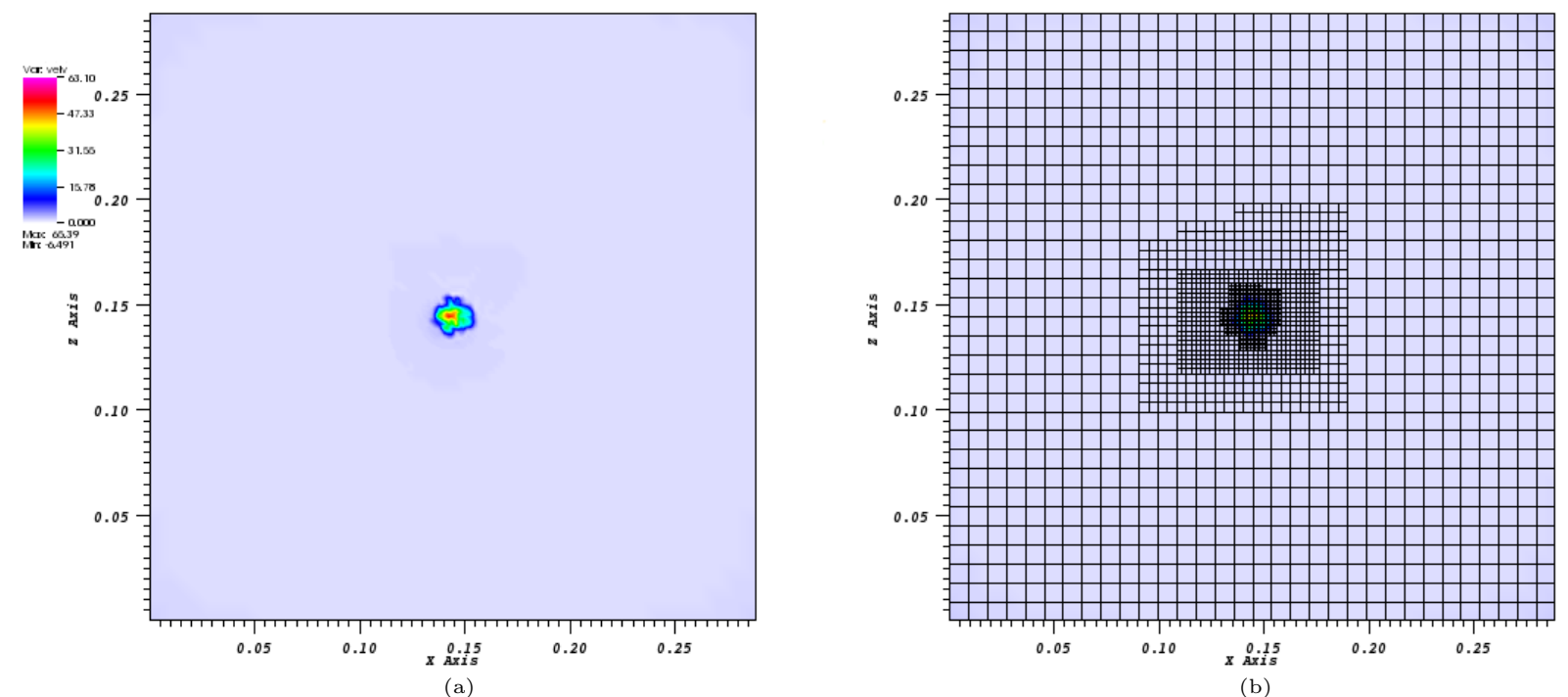

Figura 6.9: Escoamento incompressível (não-reativo) turbulento: componente da velocidade na direção do escoamento em um instante de tempo em um plano de corte transversal em $15 \%$ (a)-(b).

descrito pela expressão (6.7). Vale ressaltar que seria necessário mais um nível de refinamento para que o bocal contivesse o número mínimo de células computacionais relatado na literatura. Além disso, verificou-se que o decaimento da velocidade na linha de centro é influenciado pela escolha da constante de Smagorinsky, do modelo de turbulência. Mais comentários são descritos na Seção 6.4. 


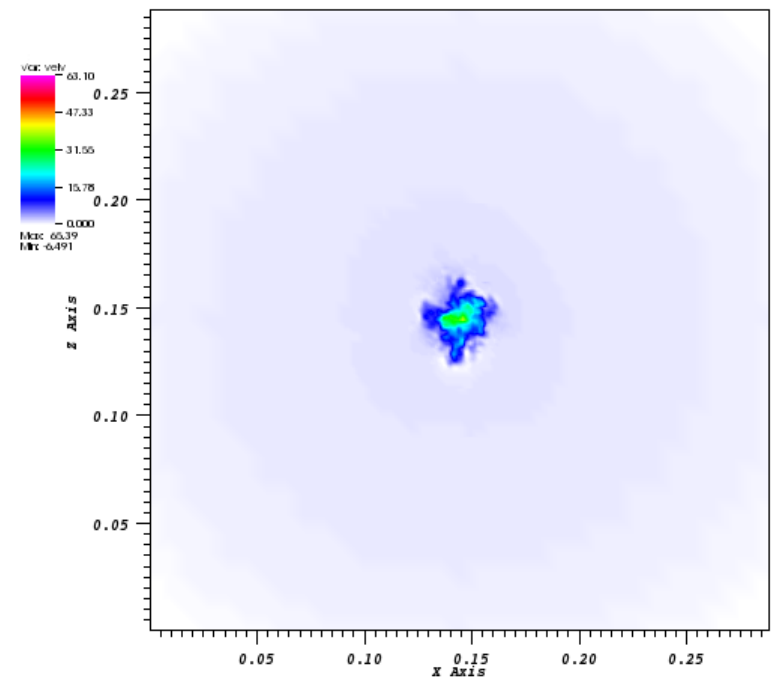

(a)

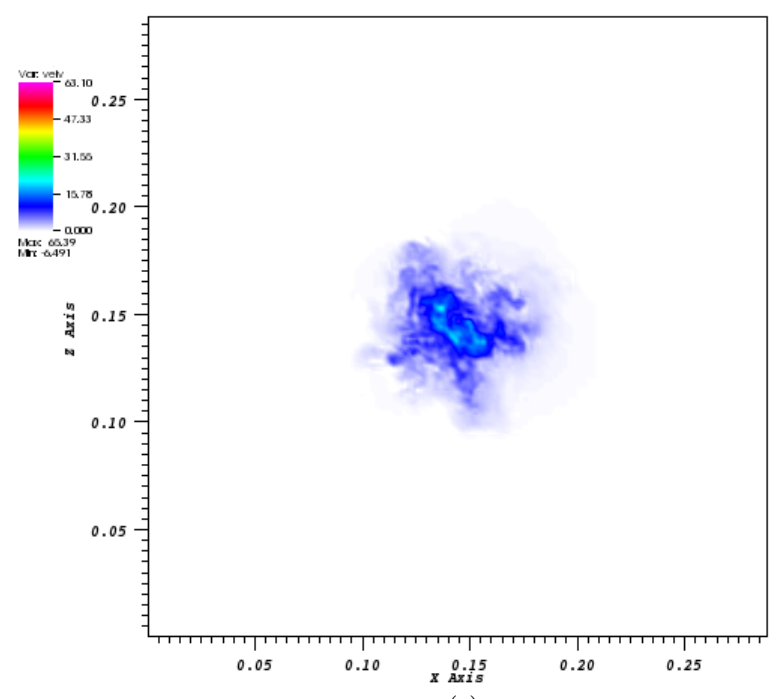

(c)

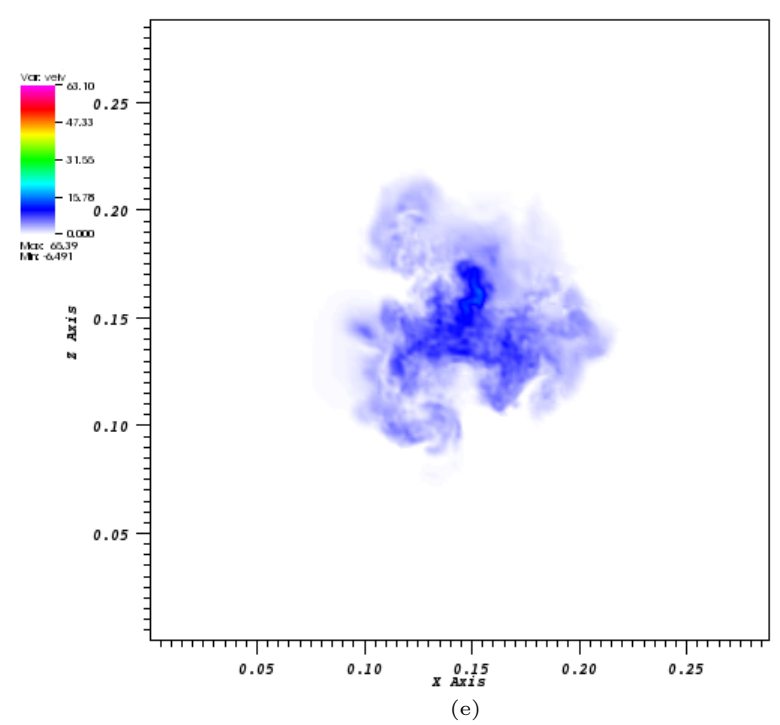

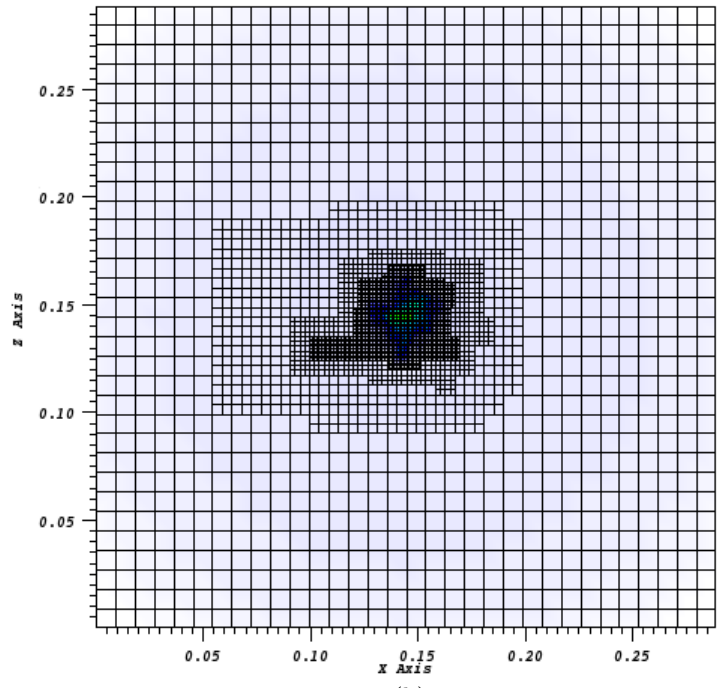

(b)

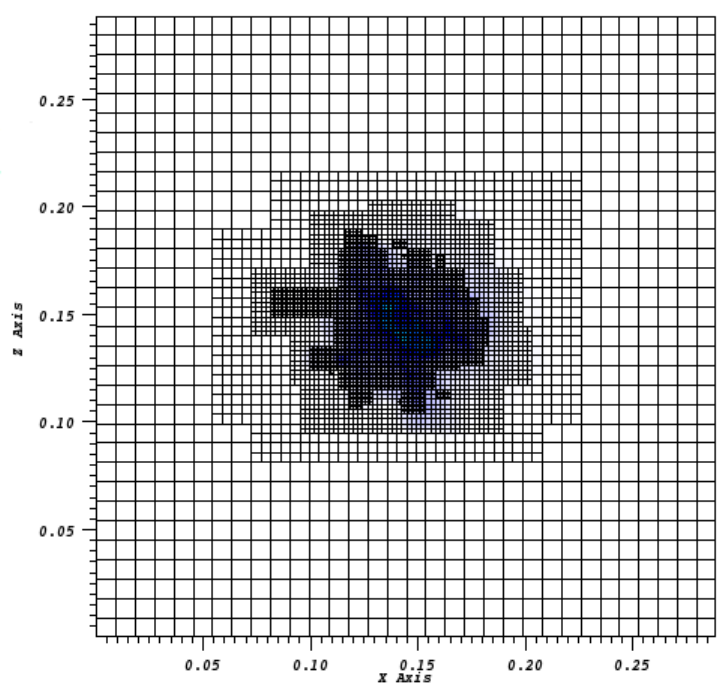

(d)

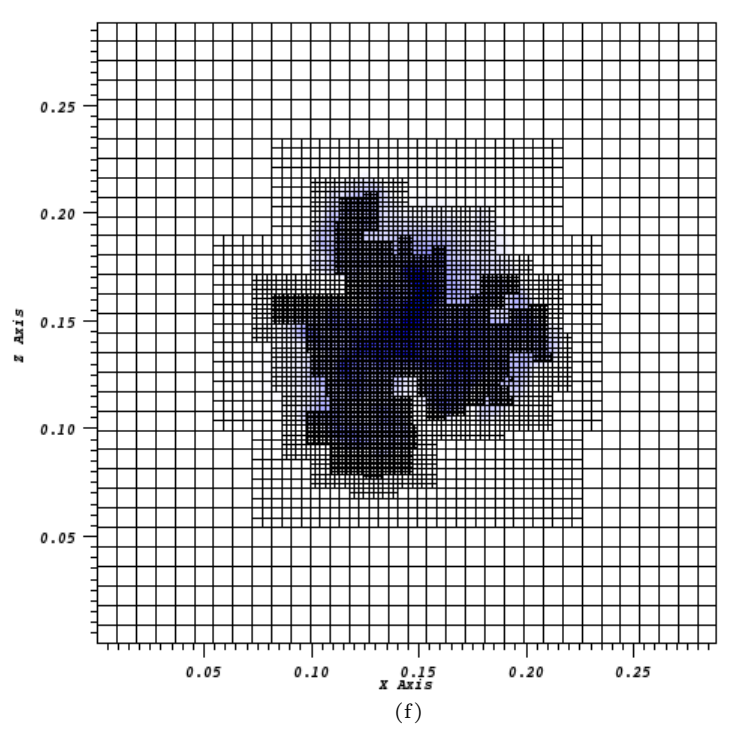

Figura 6.10: Escoamento incompressível (não-reativo) turbulento: componente da velocidade na direção do escoamento em um instante de tempo em em planos de corte transversais em $25 \%$ (a)-(b), $50 \%$ (c)-(d) e $75 \%$ (e)-(f). 


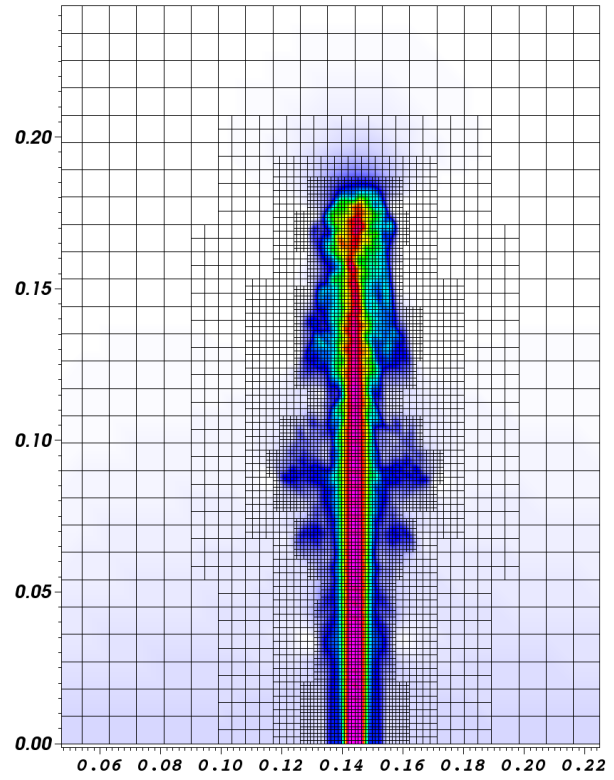

(a) Componente da velocidade e malha.

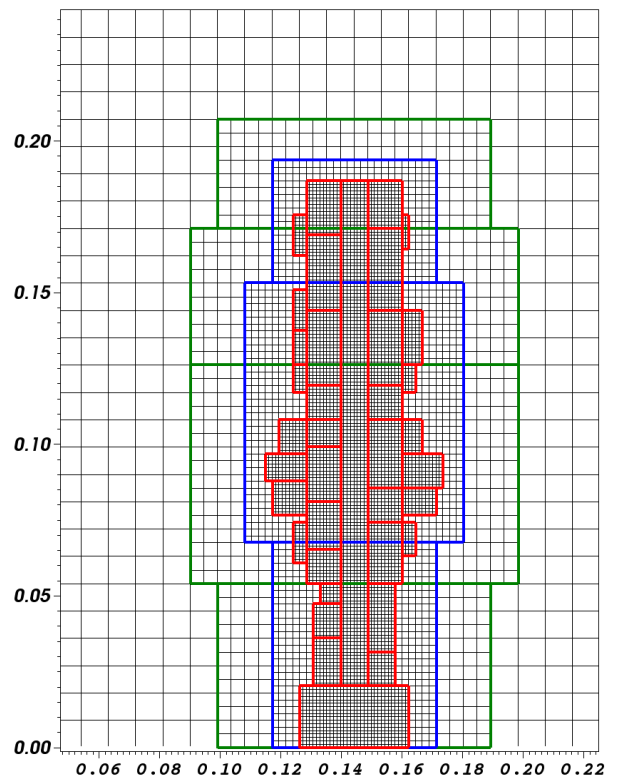

(b) Blocos discretizados e níveis de refinamento.

Figura 6.11: Detalhe de uma malha com quatro níveis de refinamento adaptativo na simulação do escoamento (não-reativo) turbulento.

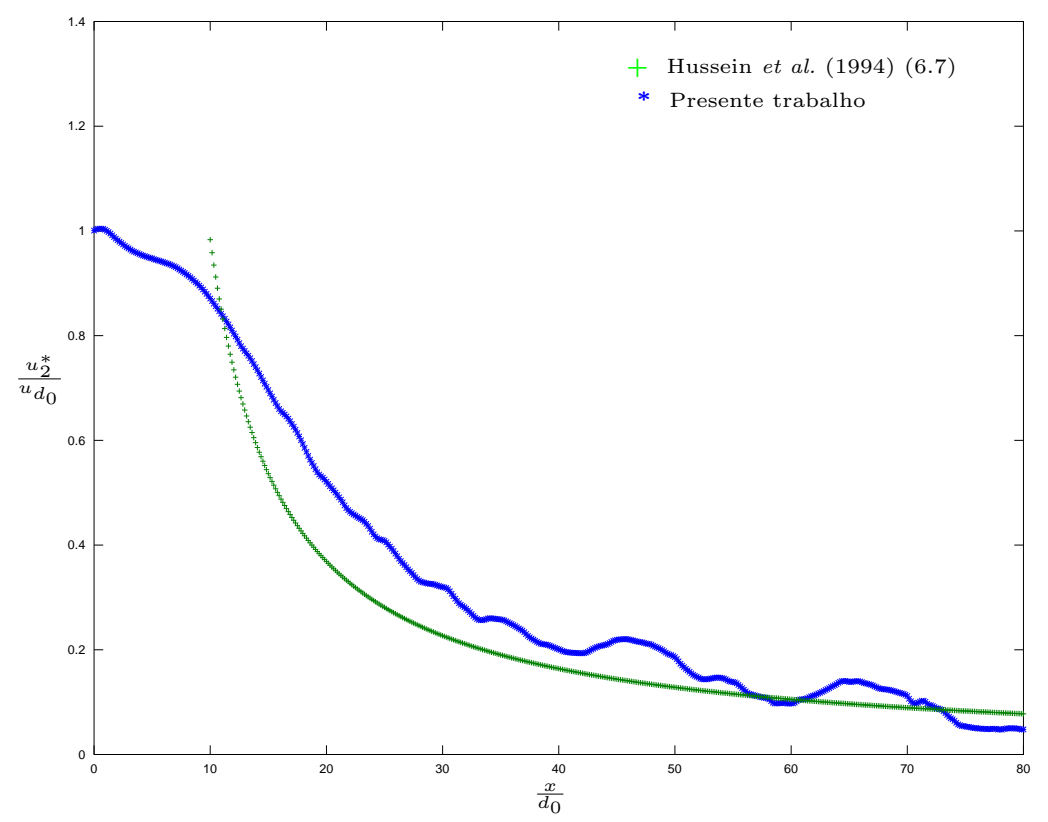

Figura 6.12: Decaimento médio da componente da velocidade na direção do escoamento na linha de centro do domínio computacional.

Para ilustrar a eficácia da modelagem da turbulência, calculou-se o espectro da energia cinética turbulenta em dois pontos do domínio computacional. A Figura 6.13(a) mostra a localização dos 
pontos escolhidos para a coleta das informações (estatísticas) para o cálculo da energia cinética turbulenta (para mais detalhes veja Pope (2000)). A Figura 6.13(b) mostra o espectro da energia cinética turbulenta nos dois pontos escolhidos. A imagem inferior se refere ao ponto $B=(1.44 \times$ $\left.10^{-1} \mathrm{~m}, 1.125 \times 10^{-1} \mathrm{~m}, 1.44 \times 10^{-1} \mathrm{~m}\right)$ próximo a saída do bocal e a imagem superior mostra o espectro da energia cinética turbulenta no ponto $A=\left(1.44 \times 10^{-1} \mathrm{~m}, 3.24 \times 10^{-1} \mathrm{~m}, 1.44 \times 10^{-1} \mathrm{~m}\right)$. Ambas as imagens mostram certa concordância com o decaimento esperado de $-\frac{5}{3}$. Um próximo passo do trabalho incluirá um modelo de turbulência dinâmico, veja Germano et al. (1991) e outras maneiras de simular a turbulência na entrada do domínio computacional.

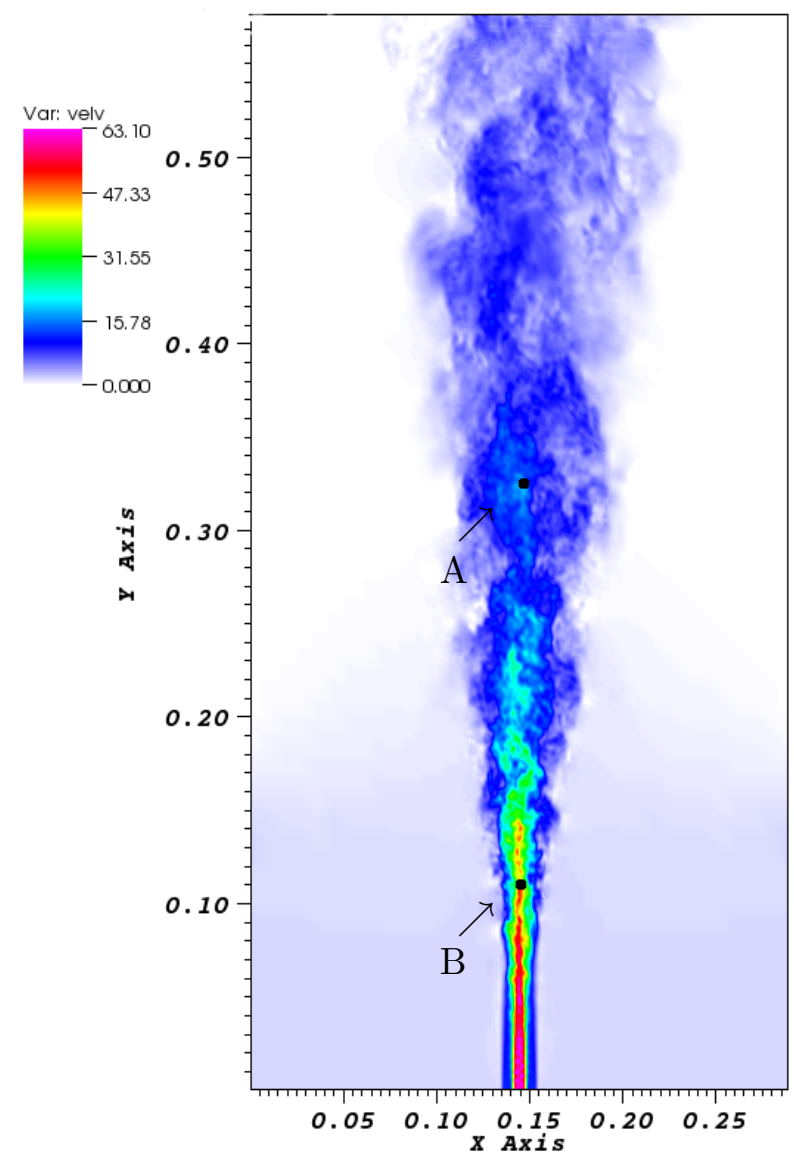

(a) Localização dos pontos escolhidos para o coleta das informações.
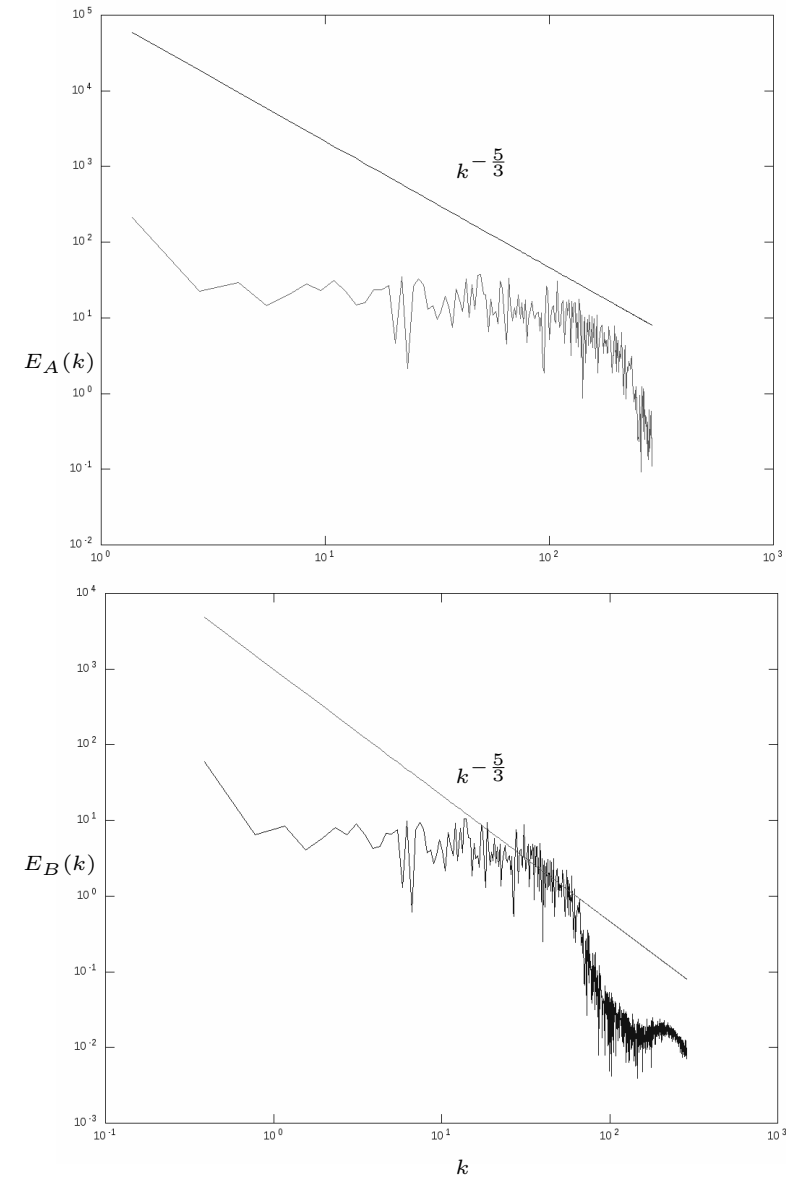

(b) Decaimento do espectro da energia cinética turbulenta.

Figura 6.13: Decaimento do espectro da energia cinética turbulenta nos pontos A e B juntamente com a reta de inclinação $-\frac{5}{3}$.

A seguir são descritos alguns aspectos da implementação que mostram as vantagens e algumas dificuldades da metodologia adotada para simular um escoamento incompressível (não-reativo) turbulento. Para comparar o benefício do uso da malha composta bloco-estruturada com refinamento adaptativo versus o uso da malha uniforme, a simulação foi realizada em uma malha com $256 \times 512 \times 256$ células computacionais, a qual é equivalente ao nível de refinamento mais fino. Comparando-se com o tempo gasto pela simulação numérica quando o escoamento já estava desenvolvido (veja a Figura 6.13(a)) com aproximadamente $8.9 \times 10^{6}$ células computacionais (incluindo células fantasmas e células de níveis de multigrid, abaixo do nível base), a simulação numérica na 
malha composta foi 5 vezes mais rápida. Vale ressaltar que a simulação numérica em uma malha uniforme possui um custo computacional constante, enquanto que para este problema de aplicação, a simulação na malha composta é muito mais rápida no início da simulação já que a quantidade de blocos discretizados é menor que no caso comparado (com o escoamento desenvolvido), chegando a ser 190 vezes mais rápida que a malha uniforme. As Figuras 6.14(a)-6.14(b) mostram a razão entre os tempos gastos na malha uniforme (TMU) e na malha composta (TMC) bloco-estruturada. A vantagem da simulação numérica na malha AMR sobre a simulação na malha uniforme está diretamente ligada ao número de células computacionais. Uma pequena porcentagem do tempo de processamento é gasto durante o processo de remalhagem (troca da malha e inicialização das variáveis), geralmente menos de $2 \%$ do tempo gasto em um passo de integração. Nesta simulação foram feitas 131 remalhagens ao longo dos $1.4 \times 10^{4}$ passos de integração.

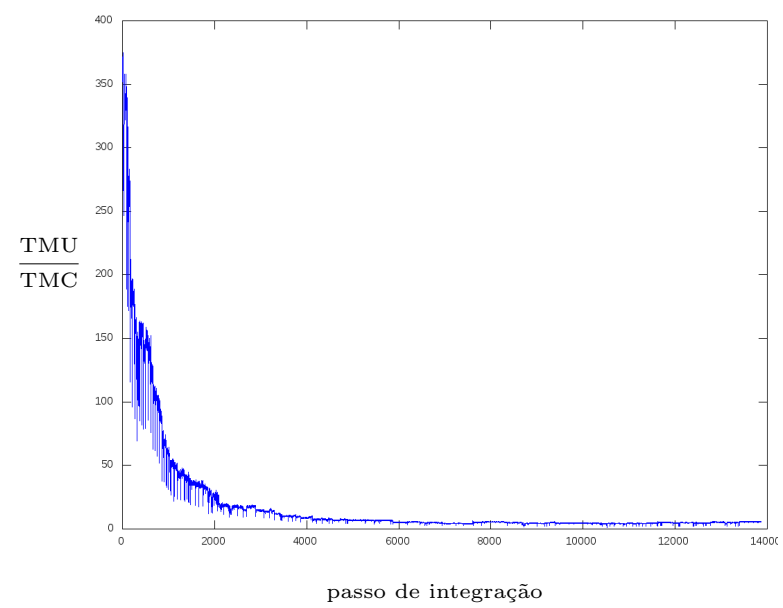

(a) Em todos os passos de integração.

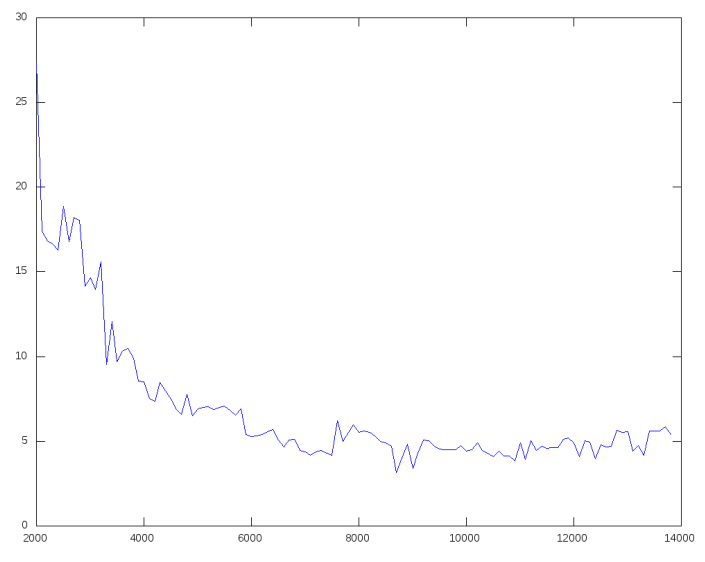

passo de integração

(b) A partir do passo de integração 2000.

Figura 6.14: Razão entre o tempo computacional gasto na malha uniforme (TMU) e na malha composta bloco-estruturada (TMC).

A respeito dos compiladores, foram medidos o tempo computacional de um único passo de integração utilizando-se os compiladores gnu (versão 4.4.4) e intel (versão 11.0) usando a flag "-O3", em uma máquina (Intel Core 2 Quad processador $2.33 \mathrm{GHz}$ e 16Gb RAM). O melhor desempenho foi obtido com o compilador da gnu o qual foi 1.2 vezes mais rápido que o compilador intel para esta simulação.

Um dos "gargalos" do atual código é a resolução da equação da correção da pressão a qual gasta $69.5 \%$ do tempo computacional em cada passo de integração. Fazendo um perfil do código, utilizando a flag "-pg" e executando o gprof é possível identificar as funções/subrotinas da implementação numérica que gastam mais tempo de processamento. Os resultados mostram que o conjunto de subrotinas envolvidas no cálculo de valores de células fantasmas gastam $43 \%$ do tempo computacional de um passo de integração. Uma razão é o grande número de blocos discretizados gerados quando o escoamento está desenvolvido. Para aumentar a eficiência, diminuiu-se a quantidade de blocos, aproveitando a estrutura do problema, substituindo-os por único bloco discretizado ao longo da direção do escoamento (um "tubo"). Esta redução de blocos é automática, sendo feita a medida que o escoamento vai se desenvolvendo. Com esta estratégia, diminuiu-se o número de chamadas para o conjunto de subrotinas que determina valores para as células fantas- 
mas. A Tabela 6.1 mostra o número de células computacionais da simulação na malha uniforme e da simulação nas malhas com refinamento adaptativo, com e sem a estratégia do "tubo". A coluna "Total" apresenta todas as células computacionais empregadas (isto é, a soma das células fantasmas, interiores, cobertas por níveis mais finos e níveis multigrid), a coluna "Interiores" apresenta o número total de células computacionais que compõem os "níveis físicos" (incluem-se células computacionais cobertas, mas não células computacionais fantasmas e de níveis multigrid) e a coluna "Visíveis" inclui apenas as células computacionais interiores e que não são cobertas por células computacionais de níveis de refinamento mais finos. Empregando-se a estratégia que diminui o número de blocos discretizados resultou em um aumento de eficiência de aproximadamente $30 \%$, o que mostra a sobrecarga de cálculos acarretada pela quantidade de blocos de refinamento.

Tabela 6.1: Número de células computacionais em diferentes malhas.

\begin{tabular}{c|c|c|c}
\hline Malha & Total & Interiores & Vísiveis \\
\hline AMR & $8.69 \times 10^{6}$ & $3.44 \times 10^{6}$ & $3.00 \times 10^{6}$ \\
AMR $($ tubo $)$ & $7.47 \times 10^{6}$ & $3.40 \times 10^{6}$ & $2.92 \times 10^{6}$ \\
Uniforme & $41.5 \times 10^{6}$ & $33.5 \times 10^{6}$ & $33.5 \times 10^{6}$ \\
\hline
\end{tabular}

\subsection{Simulação de um escoamento (não-reativo) com gotículas iner- tes}

Um dos objetivos do presente trabalho é o de explorar a metodologia numérica em desenvolvimento visando a identificação das primeiras dificuldades na simulação de gotículas (fase dispersa) em escoamentos turbulentos. A simulação computacional de escoamento contendo gotículas lagrangianas impõe uma sobrecarga computacional sobre a metodologia como um todo (García (2009) é De et al. (2011) citam entre $6 \times 10^{5}$ e $1 \times 10^{6}$ de gotículas).

As questões que surgem vão desde os modelos matemáticos mais adequados a serem empregados, (e.g. para descrever seu transporte, eventual evaporação, troca de massa e de quantidade de movimento) até as técnicas/algoritmos que devem ser consideradas para que o código computacional seja o mais eficiente possível sem perder a física do problema, (e.g. qual o nível adequado de refinamento da malha euleriana e como marcar a vizinhança de uma célula computacional que necessita de refinamento a custo computacional reduzido). Aqui, dá-se o primeiro passo para se propor um módulo lagrangiano para tratar gotículas em uma malha bloco-estruturada com refinamento adaptativo. O problema a ser considerado é o dado por um escoamento incompressível (não-reativo) turbulento e as gotículas são consideradas inertes e sem evaporação. A simulação numérica de um spray em escoamentos reativos é um problema complexo demais para ser abordado num primeiro momento (veja De et al. (2011), García (2009), Crowe et al. (1998) e Sirignano (1999)).

O problema-modelo selecionado a ser simulado numericamente é baseado no experimento realizado por Stårner e Masri (2003). No trabalho de Stårner e Masri (2003), os autores realizam experimentos em laboratório de dois jatos turbulentos (não-reativo) (em condições idênticas), com gotículas inertes: o primeiro com um spray de terebentina (turpentine, um solvente orgânico, considerado no referido trabalho como "não evaporativo", veja Gounder e Masri (2009)) e o segundo com um spray de acetona (altamente volátil o qual exigiria do modelo matemático a inclusão de um 
modelo de evaporação). O líquido pressurizado é alimentado por um nebulizador ultrasônico para produzir as gotículas dispersas. Estas gotículas são removidas do nebulizador por intermédio de um fluxo de ar através do bocal de injeção. Mais detalhes a respeito do experimento são descritos por Stårner e Masri (2003), Gounder e Masri (2009) e De et al. (2011).

Neste contexto, é descrita a seguir a simulação computacional de um jato turbulento (nãoreativo) com gotículas de terebentina. O modelo matemático para a fase contínua é aquele dado pelas equações de Navier-Stokes

$$
\begin{aligned}
\rho_{c}\left(\frac{\partial u_{i}}{\partial t}+u_{j} \frac{\partial u_{i}}{\partial x_{j}}\right) & =-\frac{\partial p}{\partial x_{i}}+\frac{\partial}{\partial x_{j}}\left[\mu\left(\frac{\partial u_{i}}{\partial x_{j}}+\frac{\partial u_{j}}{\partial x_{i}}\right)\right]+S_{u_{i}}, \text { para } i=1,2,3 \\
\frac{\partial u_{j}}{\partial x_{j}} & =0
\end{aligned}
$$

onde $\rho_{c}$ a massa específica da fase contínua, $\mu$ é a vicosidade dinâmica mais a viscosidade turbulenta $\mu_{t}$ (veja o Apêndice A), $u_{i}$ é a componente do campo de velocidade em cada uma das direções, $p$ é a pressão e $S_{u_{i}}$ contém o termo que acopla a fase dispersa à fase contínua.

A dinâmica das gotículas lagrangianas é modelada matematicamente pelo sistema de equações diferenciais ordinárias associado a cada gotícula $p$, dado por

$$
\begin{aligned}
& \frac{d U_{i, p}}{d t}=\frac{1}{\tau_{p}}\left(u_{i, p}-U_{i, p}\right)+\left(1-\frac{\rho_{c}}{\rho_{d}}\right) \mathbf{g}, \\
& \frac{d X_{i, p}}{d t}=U_{i, p}, \quad \text { para } i=1,2,3,
\end{aligned}
$$

sendo $\left(X_{1, p}, X_{2, p}, X_{3, p}\right)=\mathbf{X}_{p}$ a posição da gotícula, $\left(U_{1, p}, U_{2, p}, U_{3, p}\right)=\mathbf{U}_{p}$ a velocidade da gotícula, $\rho_{d}$ a densidade da fase líquida, $\tau_{p}$ o tempo de resposta da gotícula e $\mathbf{g}$ a aceleração da gravidade, para mais detalhes veja a Seção 2.4.1.

Os dados da simulação, descritos por Stårner e Masri (2003) e De et al. (2011), são aqui apresentados brevemente. As gotículas de terebentina entram no domínio computacional através de um do bocal de injeção de tamanho $d_{0}=10 \times 10^{-3} \mathrm{~m}$ junto com o escoamento da fase contínua à velocidade $u_{2, d_{0}}=$ $24 \mathrm{~m} / \mathrm{s}$, veja a Figura 6.15. No restante da entrada do domínio computacional a velocidade do escoamento da fase contínua é $u_{2}=3 \mathrm{~m} / \mathrm{s}$. O número de Reynolds do escoamento é $1.96 \times 10^{4}$. As dimensões do domínio computacional são dadas por $30 d_{0} \times 60 d_{0} \times 30 d_{0}$. A velocidade da fase contínua na entrada do domínio computacional é dada por um perfil tangencial

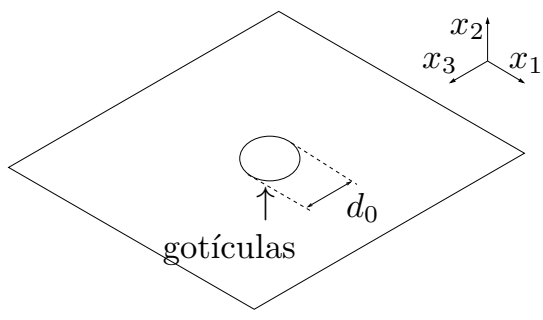

Figura 6.15: Esboço da entrada das gotículas e da componente da velocidade na direção do escoamento. (6.5), acrescido de uma perturbação de $2 \%$ (ruído branco, veja a Seção 6.1.2) para simular a turbulência. A turbulência é modelada por meio do modelo de Smagorinsky com constante $C_{s}=0.15$.

A densidade da fase líquida (das gotículas de terebentina) é $\rho=870 \mathrm{~kg} / \mathrm{m}^{3}$. A quantidade de massa líquida injetada é $m_{d, \text { in }}=5.95 \times 10^{-5} \mathrm{~kg} / \mathrm{s}$. As gotículas são distribuídas uniformemente em um disco de raio $r_{0}=d_{0} / 2$ no plano $x_{2}=0.3 \times d_{0}$, como mostra a Figura 6.16 , conforme Stårner e Masri (2003). A velocidade inicial da gotículas na direção do escoamento é dada pela velocidade $u_{2, d_{0}}$ acrescida de uma perturbação de $1 \%$. 


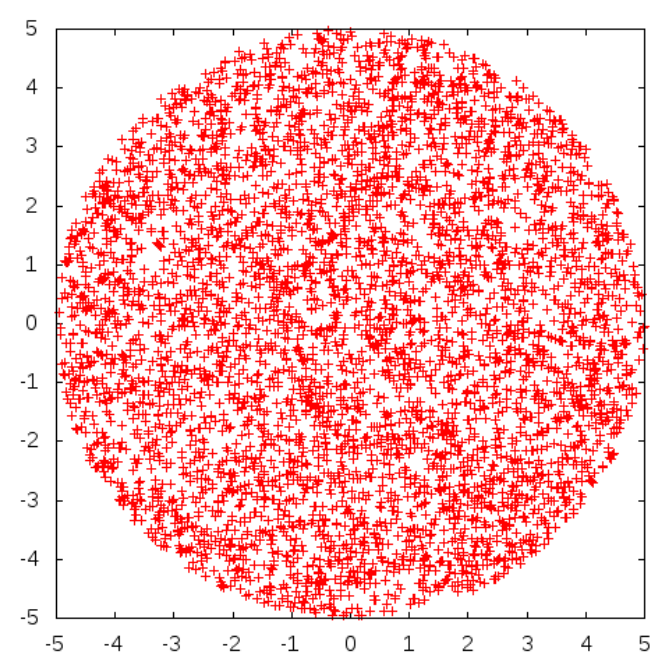

Figura 6.16: Distribuição uniforme sobre um disco.

O diâmetro médio das gotículas é $d_{p, M}=40 \mu \mathrm{m}$ e as gotículas são distribuidas por meio de uma função distribuição de probabilidade. São exemplos de distribuições de probabilidade as funções Gaussiana, log-normal, Nukiyama-Tanasawa e a log-hiperbólica, veja Crowe et al. (1998), Bhatia e Durst (1989) e Lefebvre (1989). No presente trabalho, inicialmente, emprega-se a distribuição log-normal a qual frequentemente é utilizada para representar gotículas, veja García (2009).

A distribuição log-normal é obtida a partir de uma distribuição normal. Se y possui uma distribuição log-normal, então $x=\ln (y)$ possui uma distribuição normal. A função densidade de probabilidade de uma distribuição log-normal é dada por

$$
f\left(x ; \mu_{o}, \sigma_{o}\right)=\frac{1}{x \sigma_{o} \sqrt{2 \pi}} \exp \left(-\frac{\ln x-\mu_{o}}{2 \sigma_{o}^{2}}\right),
$$

sendo os parâmetros $\sigma_{o}$ e $\mu_{o}$ são obtidos a partir da média $d_{p, M}$ e da variância var por meio das expressões,

$$
\mu_{o}=\ln \left(\frac{d_{p, M}^{2}}{\sqrt{v a r+d_{p, M}^{2}}}\right) \text { e } \sigma_{o}=\sqrt{\ln \left(\frac{v a r}{d_{p, M}^{2}}+1\right)} .
$$

Para mais detalhes veja Mood et al. (1974). A Figura 6.17 mostra a distribuição log-normal do diâmetro das gotículas lagrangianas. O número de gotículas $N_{p, i n}$ injetadas por passo no tempo depende da quantidade de massa da fase líquida que entra no domínio computacional,

$$
m_{d, i n} \Delta t=\sum_{p=1}^{N_{p, i n}} \rho_{d} \frac{d_{p}^{3}}{6} \pi
$$

onde $\Delta t$ é o tamanho do passo de integração e $d_{p}$ é o diâmetro da gotícula $p$.

A simulação computacional é realizada em uma malha bloco-estruturada composta por uma malha base mais três níveis de refinamento adaptativo (quatro níveis de refinamento no total). A malha base possui $32 \times 64 \times 32$ células computacionais. O espaçamento do nível mais fino é $\Delta x=1.17 \times 10^{-3} \mathrm{~m}$. O que garante oito células computacionais na região do bocal. Os critérios de 


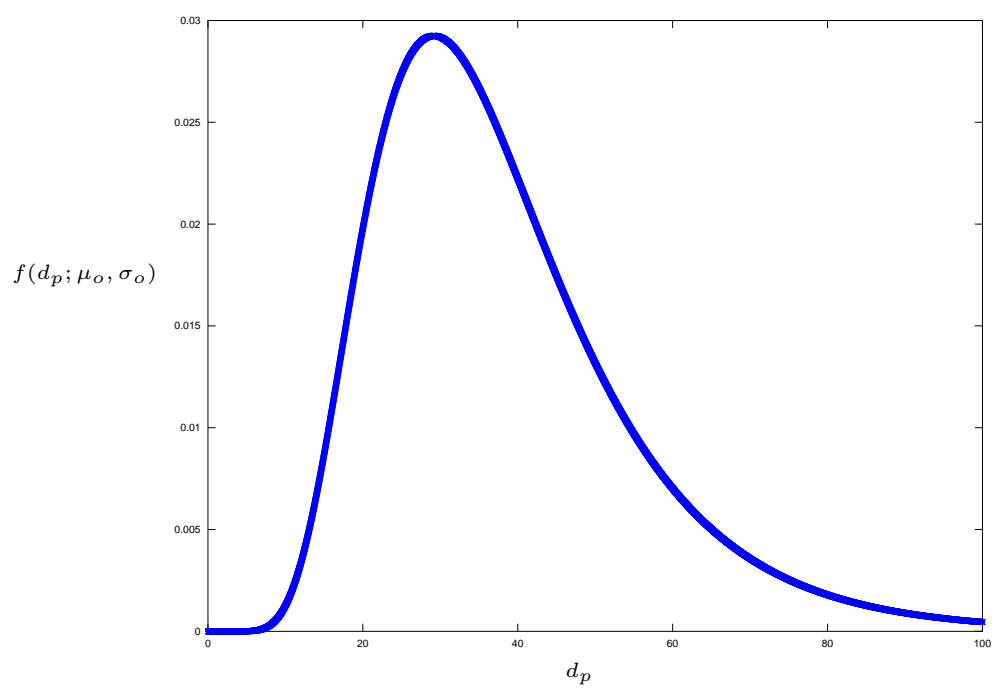

Figura 6.17: Distribuição log-normal para o diâmetro das gotículas.

refinamento utilizados para a fase contínua são os já descritos na Seção 6.1.2. Além destes critérios, nesta simulação assume-se que todas as gotículas devem estar no nível mais fino de refinamento, veja a Seção 4.2 (um critério bastante exigente que facilita a identificação de possíveis dificuldades de cunho computacional). A Figura 6.18 mostra a malha bloco-estruturada gerada em dois instantes de tempo diferentes e a velocidade do escoamento, em um corte transversal em $45 \%$ do domínio computacional na direção do escoamento. Os contornos identificam os blocos discretizados que compõem cada um dos quatro níveis de refinamento. As Figuras 6.19(a)-(d) e 6.20(a)-(b) mostram a evolução da velocidade das gotículas em um plano de corte no centro do domínio computacional.
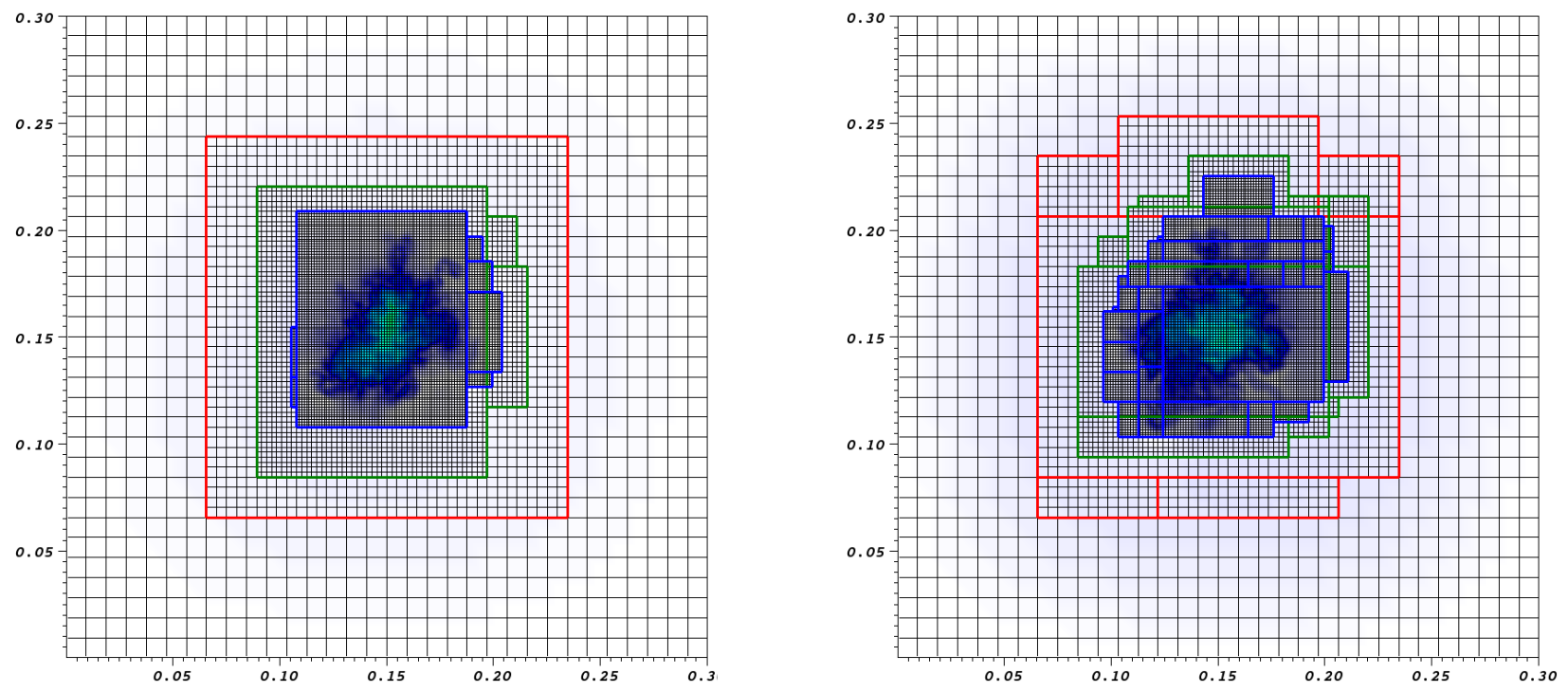

Figura 6.18: Velocidade na direção do escoamento e malha bloco-estruturada gerada na simulação do escoamento com gotículas em um corte transversal. 


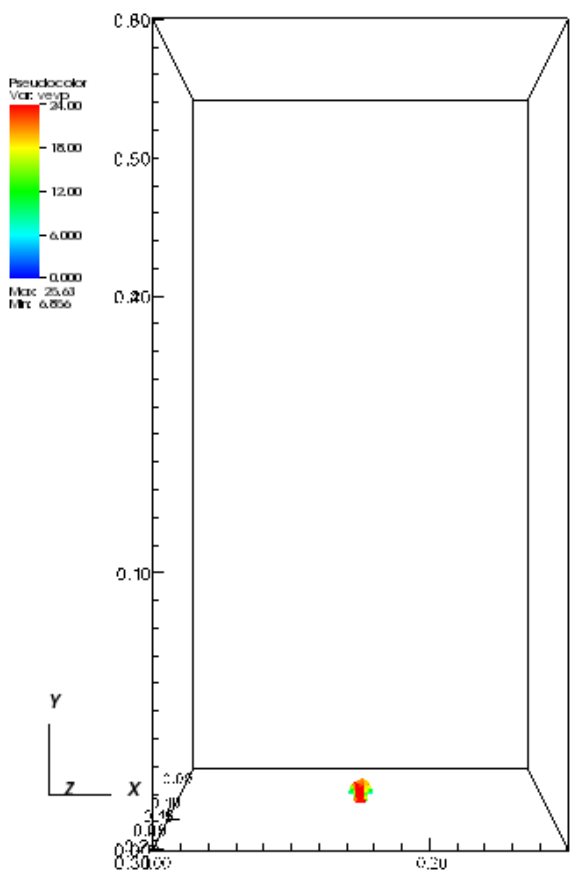

(a)

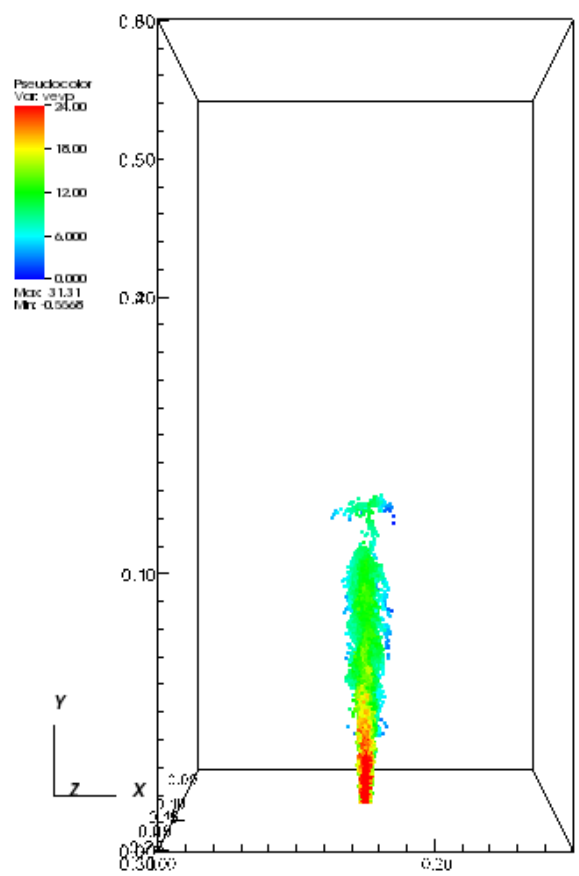

(c)

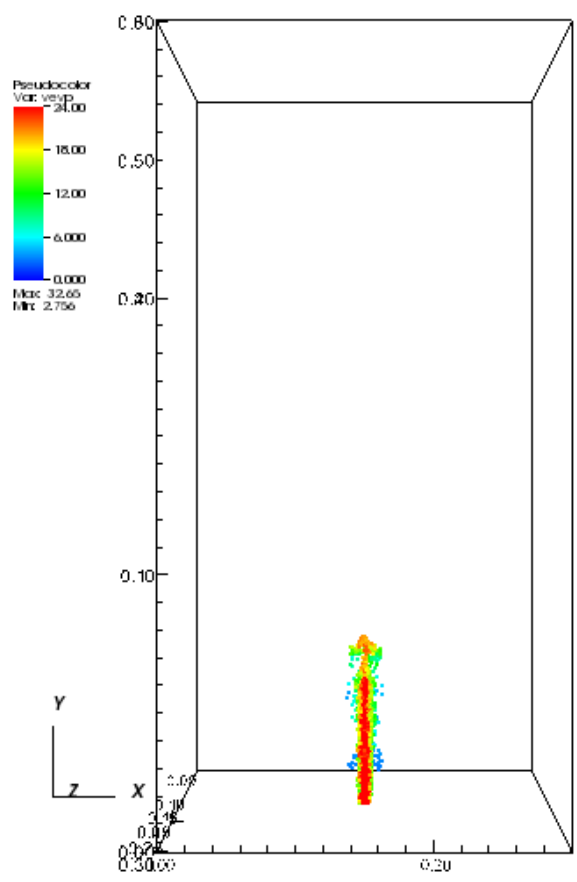

(b)

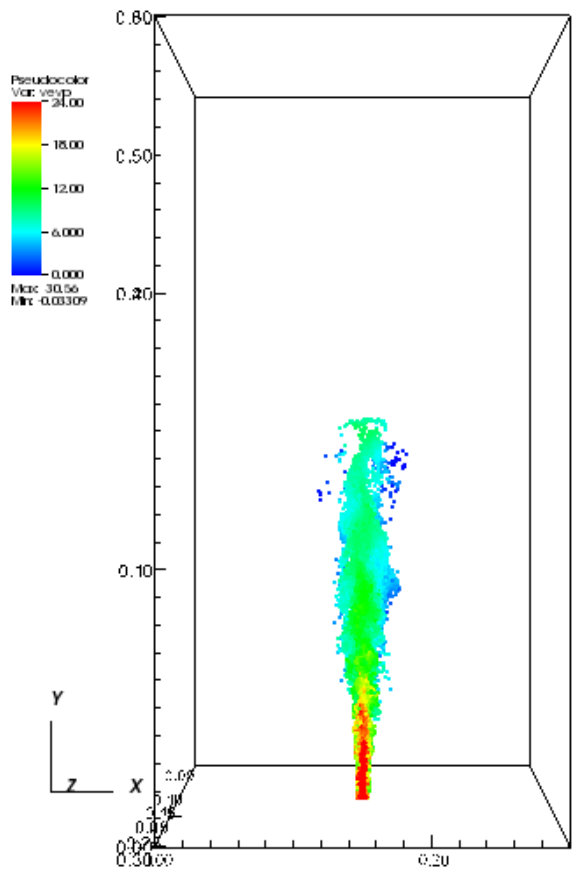

(d)

Figura 6.19: Escoamento incompressível (não-reativo) turbulento com gotículas inertes: (a)-(d) velocidade das gotículas na direção do escoamento com um plano de corte central.

Na simulação numérica de um spray algumas gotículas podem estar na mesma célula computacional. Com isso pode acontecer de uma célula computacional ser selecionada para refinamento mais de uma vez. Desta maneira durante a seleção de células computacionais para refinamento tomou-se cuidado para que uma célula computacional não fosse marcada mais que uma vez. Além disso, não havendo interação entre as gotículas espera-se ganhar muito em eficiência por paralelização deste módulo.

Para comparar os resultados da simulação numérica com os resultados experimentais faz-se 


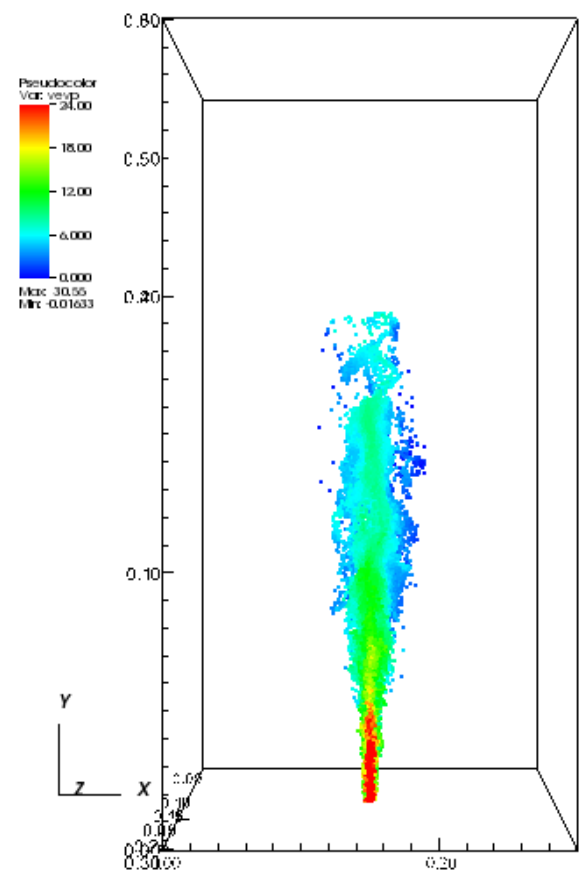

(a)

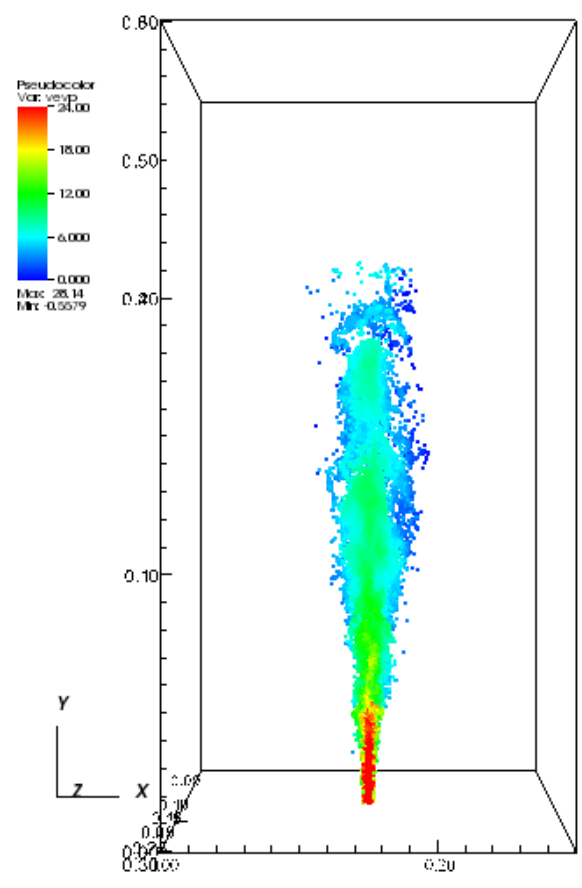

(b)

Figura 6.20: Escoamento incompressível (não-reativo) turbulento com gotículas inertes: (a)-(b) velocidade das gotículas na direção do escoamento em um plano de corte central.

necessária a comparação do perfil da velocidade das gotículas nas linhas radiais do domínio computacional, como por exemplo, $x_{2}=20 \times d_{0}$. Para tanto, é necessário um tempo maior de simulação para o levantamento das estatísticas de velocidade e posicionamento das gotículas. A Figura 6.21 mostra o perfil da velocidade das gotículas (antes do escoamento atingir o regime permanente) na linha radial $x_{2}=20 \times d_{0}$. Uma das explicações para a diferença entre o perfil da velocidade das

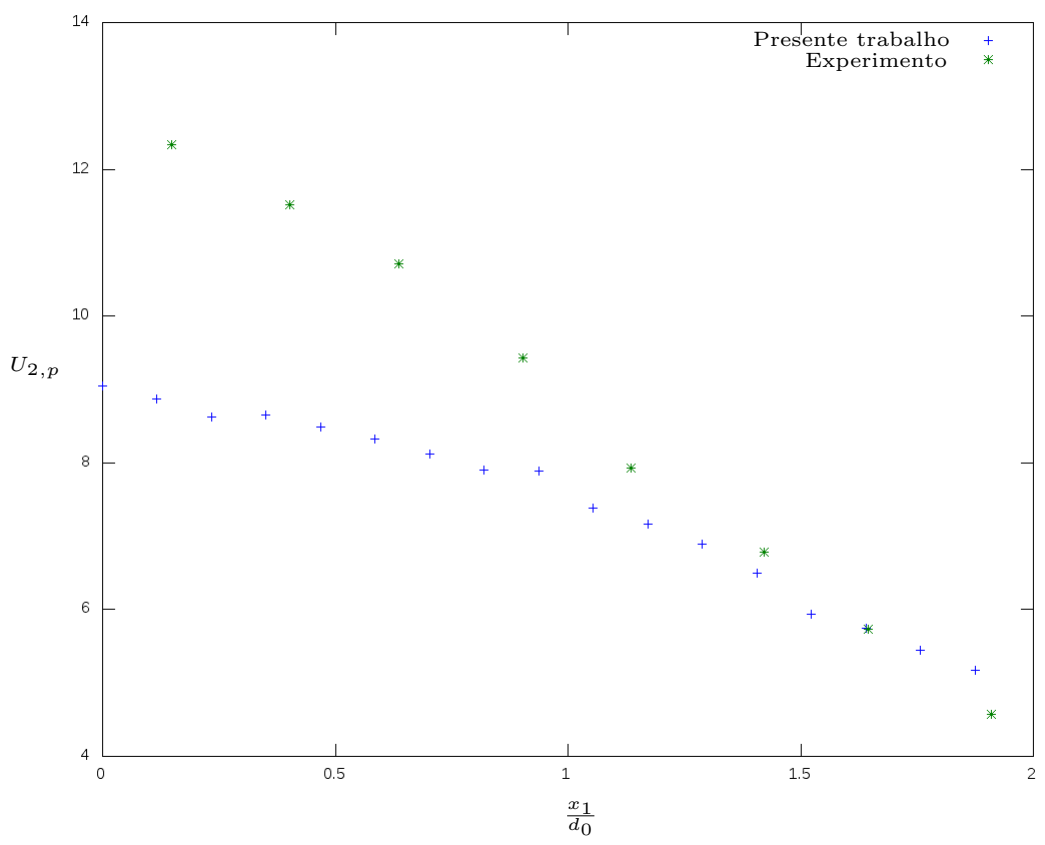

Figura 6.21: Perfil da velocidade das gotículas na linha radial. 
gotículas do experimento e da simulação é o fato de que seriam necessários pelo menos mais dois níveis de refinamento para que a velocidade do escoamento seja bem descrita no bocal de injeção, veja De et al. (2011). Este aumento no número de níveis de refinamento, no momento, tornaria a simulação inviável, veja mais comentários na Seção 6.4.

A respeito do acoplamento entre as fases, a vantagem da utilização da Delta de Dirac para aproximar os operadores responsáveis pela troca de informações entre as fases (veja a Seção 3.3.2) é que as células computacionais vizinhas a célula computacional que contém a gotícula também recebem influência da mesma e vice-versa. Neste contexto, o uso desta aproximação possui seu grau de originalidade.

Futuramente, pretende-se avaliar cuidadosamente a precisão desta aproximação no caso em que as gotículas estejam em diferentes níveis de refinamento. Além de outras distribuições de probabilidade para o tamanho das gotículas que podem ser encontradas na literatura, por exemplo, Lefebvre (1989), Crowe et al. (1998) e Bhatia e Durst (1989).

\subsection{Simulação de escoamentos reativos a baixo Mach}

A metodologia numérica proposta para a fase contínua, descrita na Seção 4.5 e que estende a capacidade do código AMR3D original, é aqui aplicada a dois escoamentos reativos: um laminar, descrito por Smooke et al. (1996) e simulado numericamente por Day e Bell (2000) e Northrup e Groth (2009), e um turbulento (baseado na Chama D), descrito por Balow e Frank (2007) e simulado numericamente por Kempf et al. (2005) e Pitsch e Steiner (2000). A Figura 6.22 mostra como ocorre a entrada do combustível e do oxidante na região de reação em uma chama não pré-misturada. As simulações computacionais são feitas empregando-se o modelo matemático para a fase contínua, descrito na Seção 2.6.3, dado pelas equações para um escoamento reativo com baixo número de Mach,

$$
\begin{aligned}
\frac{\partial \rho}{\partial t}+\frac{\partial\left(\rho u_{j}\right)}{\partial x_{j}} & =0 \\
\frac{\partial(\rho z)}{\partial t}+\frac{\partial\left(\rho u_{j} z\right)}{\partial x_{j}} & =\frac{\partial}{\partial x_{j}}\left(\rho D \frac{\partial z}{\partial x_{j}}\right) \\
\rho\left(\frac{\partial u_{i}}{\partial t}+u_{j} \frac{\partial u_{i}}{\partial x_{j}}\right) & =-\frac{\partial p_{2}}{\partial x_{i}}+\frac{\partial}{\partial x_{j}}\left[\mu\left(\frac{\partial u_{i}}{\partial x_{j}}+\frac{\partial u_{j}}{\partial x_{i}}\right)\right], \\
\frac{\partial u_{j}}{\partial x_{j}} & =S
\end{aligned}
$$

onde $\rho$ é a massa específica da mistura, $\mathbf{u}=\left(u_{1}, u_{2}, u_{3}\right)$ é o campo de velocidade, $z$ é o escalar conservado, $p_{2}$ é a pressão dinâmica, $D$ é o coeficiente de difusão das espécies químicas envolvidas na reação e $S$ é a restrição para o divergente do campo de velocidade, dada por

$$
S=\frac{1}{\rho T}\left(\frac{\partial(\rho T)}{\partial t}+\frac{\partial\left(\rho u_{j} T\right)}{\partial x_{j}}\right)+\frac{W}{\rho} \sum_{l}\left[\frac{1}{W_{l}}\left(\frac{\partial\left(\rho Y_{l}\right)}{\partial t}+\frac{\partial \rho u_{j} Y_{l}}{\partial x_{j}}\right)\right]
$$

para $l=C, O, P$ sendo $T$ a temperatura do escoamento, $W$ o peso molecular da mistura, $Y_{l}$ a fração mássica da espécie química $l$ e $W_{l}$ o peso molecular da espécies química $l$. A dedução da expressão (6.19) encontra-se na Seção 3.1.1. As espécies químicas $Y_{C}, Y_{O}$ e $Y_{P}$ e a temperatura $T$ 
são obtidas por meio da estrutura de chama de Burke-Schumann descrita na Seção 2.3.

Conforme Pember et al. (1998), os termos difusivos $\mu, D$ e $\kappa$ são, em geral, considerados funções que dependem de $\rho, T$ e $Y_{l}$. Para as simulações apresentadas, a viscosidade dinâmica $\mu$ é dada por

$$
\mu=\mu_{0}\left(\frac{T}{T_{0}}\right)^{0.7}
$$

onde $\mu_{0}=1.85 \times 10^{-5} \mathrm{~kg} / \mathrm{m} s$ e $T_{0}=298 \mathrm{~K}$. O termo $\rho D$ e a viscosidade dinâmica $\mu$, estão relacionados por meio do número de Lewis, $L e$ (veja Seção 2.3). Ao assumir-se $L e=1$, a qual é uma aproximação razoável já que Le é aproximadamente 1 para muitos gases (para mais detalhes veja Pember et al. (1998) e Kuo (2005)), obtém-se

$$
\rho D=\frac{\mu}{\operatorname{Pr}}
$$

onde $\operatorname{Pr}=0.75$ (veja Pember et al. (1998)) é o número de Prandtl.

$\mathrm{Na}$ entrada do domínio computacional, são usadas a condição de fronteira Dirichlet para as componentes do campo de velocidade, para a massa específica e para o escalar conservado e a condição de fronteira Neumann nula para a correção da pressão. Nas demais faces do domínio computacional, são usadas as condições de fronteira Neumann nulas para as componentes do campo de velocidade para a massa específica e para o escalar conservado e a condição de fronteira Dirichlet para a pressão, conforme descrevem Day e Bell (2000), Pitsch e Steiner (2000) e Balow e Frank (2007).

Na Seção 6.3.1, são apresentados os resultados numéricos de uma simulação de um escoamento laminar reativo e na Seção 6.3.2, os resultados de uma simulação de um escoamento turbulento reativo.

\subsubsection{Escoamento reativo laminar}

A simulação do escoamento reativo laminar foi realizada com base nos dados de uma chama de difusão metano-ar, estudada por Smooke et al. (1996), Day e Bell (2000) e Northrup (2004). O domínio computacional possui $0.1 m \times 0.1 m \times 0.1 \mathrm{~m}$. O diâmetro do bocal de injeção de combustível é $d_{0}=4 \times 10^{-3} \mathrm{~m}$. O combustível (uma mistura de $65 \% \mathrm{~N}_{2}$, nitrogênio, e $35 \% \mathrm{CH}_{4}$, metano), é injetado no domínio computacional por meio das frações mássicas, $Y_{C H_{4}}^{\star}=0.5149 \mathrm{e}$ $Y_{N_{2}, C}=0.4851$, à temperatura ambiente $T_{C}^{\star}=298 \mathrm{~K}$, com o perfil de velocidade

$$
u_{2}(\mathbf{x}, t)=\frac{0.7}{2}-\frac{0.7}{2} \tanh \left[25\left(\frac{r_{j}}{r_{0}}-\frac{r_{0}}{r_{j}}\right)\right] \mathrm{m} / \mathrm{s},
$$

onde $r_{j}=\sqrt{\left(x_{3}-0.5 \cdot b_{3}\right)^{2}+\left(x_{1}-0.5 \cdot b_{1}\right)^{2}}, r_{0}=2 \mathrm{~mm}$ é o raio do bocal de injeção do combustível e $b_{1}$ e $b_{3}$ são os tamanhos do domínio computacional nas direções $x_{1}$ e $x_{3}$, respectivamente.

$\mathrm{O}$ ar é injetado no domínio computacional por meio das frações mássicas $Y_{\mathrm{O}_{2}}^{\star}=0.232$ e $Y_{N_{2}, O}=$ 0.768 , à temperatura ambiente $T_{O}^{\star}=298 \mathrm{~K}$ e à velocidade $u_{2}(\mathbf{x}, t)=0.35 \mathrm{~m} / \mathrm{s}$. As componentes da velocidade $u_{1}$ e $u_{2}$ são nulas. Os números de Reynolds e de Mach do escoamento, respectivamente, $R e=169$ e $M a=1.6 \times 10^{-3}$. As Figuras 6.23(a)-(d) mostram, em um corte no centro do domínio computacional em $x_{3}=5 \times 10^{-2} \mathrm{~m}$, a evolução da temperatura do escoamento e as malhas adaptativas correspondentes. 


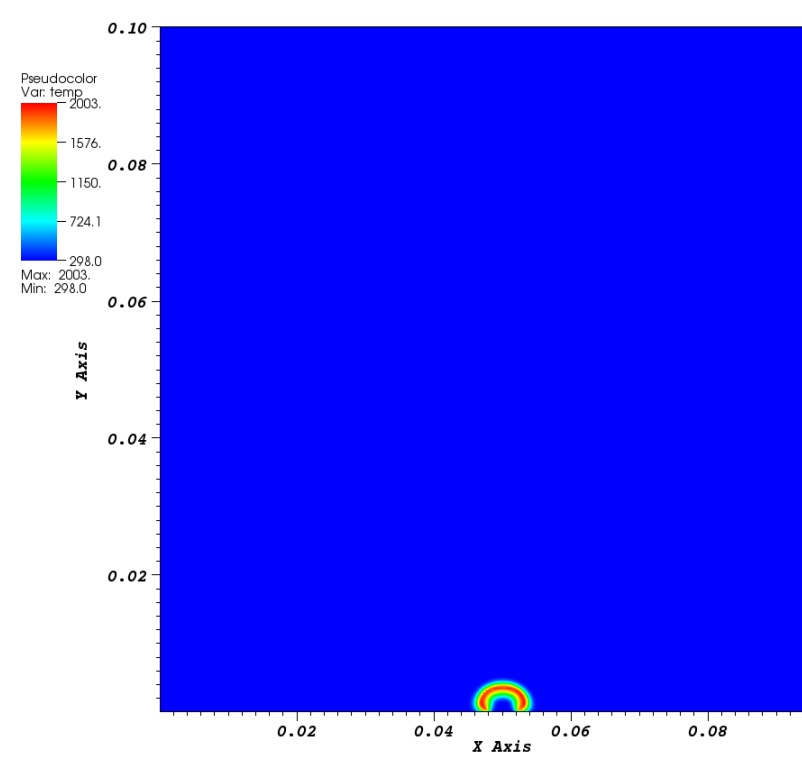

(a)

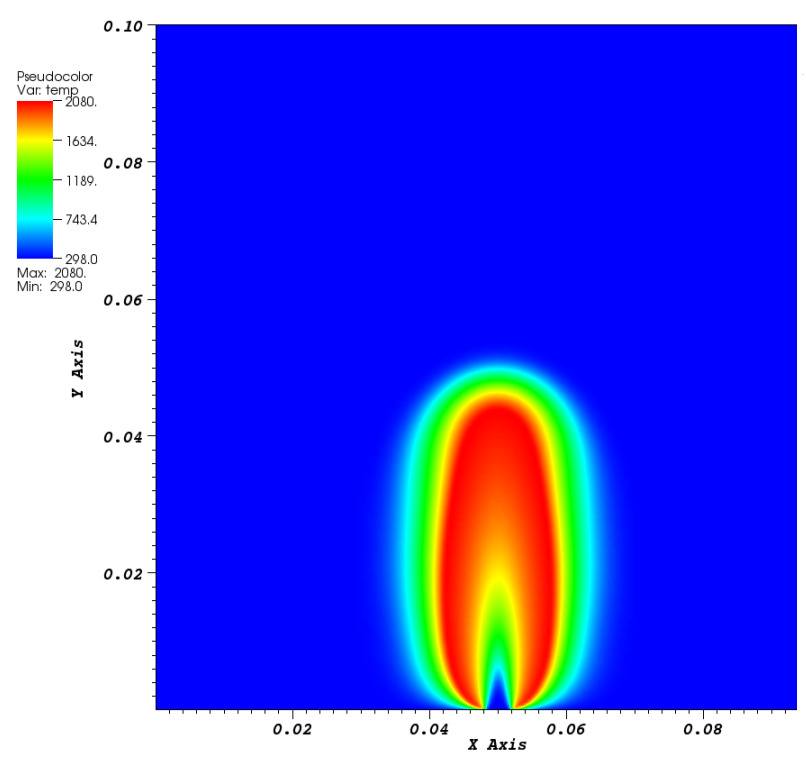

(c)

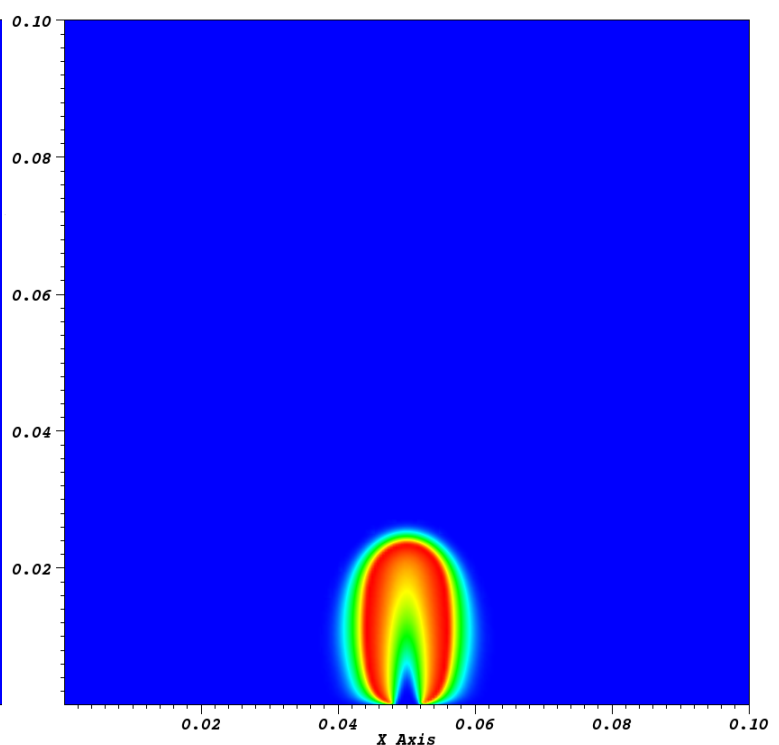

(b)

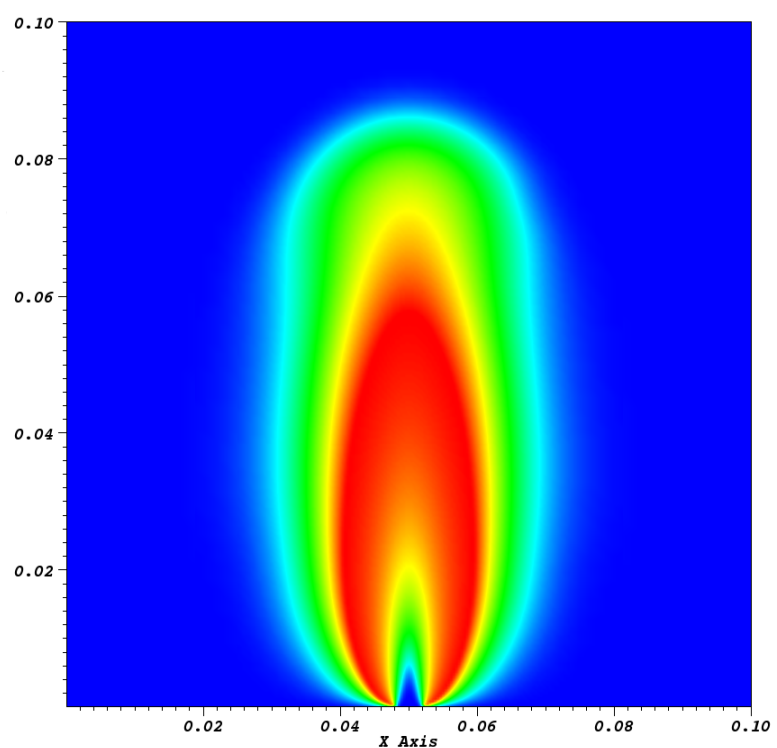

(d)

Figura 6.23: Escoamento reativo laminar: temperatura do escoamento e malhas adaptativas em um plano de corte em $x_{3}=5 \times 10^{-2} \mathrm{~m}$.

A simulação computacional foi realizada em uma malha bloco-estruturada composta por uma malha base mais três níveis de refinamento adaptativos (quatro níveis de refinamento no total). A malha base possui $32 \times 32 \times 32$ células computacionais. O espaçamento do nível de refinamento mais fino é $\Delta x=3.9063 \times 10^{-4} \mathrm{~m}$, o que garante que o diâmetro do bocal contenha pelo menos 10 células computacionais. O critério de refinamento é baseado na norma do tensor de tensões viscosas e na temperatura. A marcação das células computacionais para o refinamento adaptativo é descrita na Seção 4.2. O tamanho do passo de integração é determinado pela condição CFL (4.1) sendo, tipicamente, dado por $\Delta t=\mathrm{O}\left(\Delta x^{1.14}\right)$.

Uma comparação qualitativa feita com os trabalhos de Day e Bell (2000), Smooke et al. (1996) e Northrup (2004) mostra que os resultados obtidos concordam com resultados encontrados na li- 
teratura. A simulação numérica descrita no presente trabalho, determina uma temperatura máxima de 2083 K. A simulação de Smooke et al. (1996) utiliza um mecanismo cinético com 26 espécies e 83 reações químicas em um domínio computacional bidimensional no qual determina uma temperatura máxima de $2025 \mathrm{~K}$. A simulação numérica de Day e Bell (2000) utiliza o mesmo mecanismo cinético descrito por Smooke et al. (1996) e o mesmo domínio computacional com refinamento adaptativo, no qual determina uma temperatura máxima de 2029 K. Já Northrup (2004) utiliza um mecanismo cinético de dois passos com o mesmo domínio computacional e com refinamento adaptativo, determinando uma temperatura máxima de $2214 \mathrm{~K}$ e justifica a diferença valores ao uso do mecanismo cinético simplificado. A Tabela 6.2 apresenta as temperaturas máximas obtidas em cada um dos trabalhos. O mecanismo cinético utilizado no presente trabalho, com as hipóteses de química instantânea e estequiométrica, não capta o descolamento da chama do bocal (lift-off).

Tabela 6.2: Temperatura máxima da chama laminar.

\begin{tabular}{c|c|c|c}
\hline Smooke et al. $(1996)$ & Day e Bell (2000) & Northrup (2004) & Presente Trabalho \\
\hline $2025 \mathrm{~K}$ & $2028 \mathrm{~K}$ & $2214 \mathrm{~K}$ & $2083 \mathrm{~K}$ \\
\hline
\end{tabular}

\subsubsection{Escoamento reativo turbulento}

Escoamentos reativos turbulentos compõem a classe de problemas para os quais desenvolveu-se e intenciona-se empregar a metodologia proposta. Para iniciar a investigação de seu potencial, tal metodologia é empregada a um problema modelo baseado numa chama de difusão. A simulação numérica aqui apresentada é baseada nos dados experimentais da chama de difusão são descritos por Balow e Frank (2007) e simulações numéricas foram feitas por Pitsch e Steiner (2000) e Kempf et al. (2005). O combustível é uma mistura composta por $25 \%$ de $\mathrm{CH}_{4}$, metano e $75 \%$ de ar. O domínio computacional é o mesmo descrito para o jato turbulento (não-reativo), descrito na Seção 6.1.2, bem como o perfil da velocidade na entrada do domínio computacional, veja (6.5)(6.6). O diâmetro do bocal é $d_{0}=7.2 \times 10^{-3} \mathrm{~m}$ e o diâmetro da região piloto é $d_{1}=1.8 \times 10^{-2} \mathrm{~m}$, conforme mostra a Figura 6.22. As dimensões do domínio computacional nesta simulação são $40 d_{0} \times 80 d_{0} \times 40 d_{0}$, aproximadamente $0.288 m \times 0.576 m \times 0.288 m$.

O número de Reynolds baseado no diâmetro do bocal $d_{0}$ é $R e=2.24 \times 10^{4}$. Emprega-se uma malha bloco-estruturada composta pela malha base e mais três níveis de refinamento adaptativo (quatro níveis de refinamento no total, veja um exemplo na Figura 6.6). A malha base possui $32 \times 64 \times 32$ células computacionais. O nível mais fino possui espaçamento $1.125 \times 10^{-3} \mathrm{~m}$, o que garante que o diâmetro do bocal contenha pelo menos 6 células computacionais (na prática, isto é muito pouco, insuficiente para capturar o fenômeno. Boersma et al. (1998) descreve que 12 células são, no mínimo, suficientes. De et al. (2011) descrevem que 24 células são, no mínimo, suficientes para escoamentos com gotículas). Este é o preço que o código estendido, sendo sequencial pode pagar. Mais comentários a respeito do número de células computacionais são feitos na Seção 6.4.

O critério de refinamento usado nesta simulação foi baseado na norma da vorticidade, na norma da viscosidade turbulenta e na temperatura. A marcação das células computacionais para a criação da nova malha bloco-estruturada com refinamento adaptativo é descrita na Seção 4.2. Dentre os três critérios, a viscosidade turbulenta é o critério mais exigente. A Figura 6.24 mostra a temperatura do escoamento e a malha bloco-estruturada gerada em dois instantes de tempo diferentes em um 
corte transversal na metade do domínio computacional na direção do escoamento.
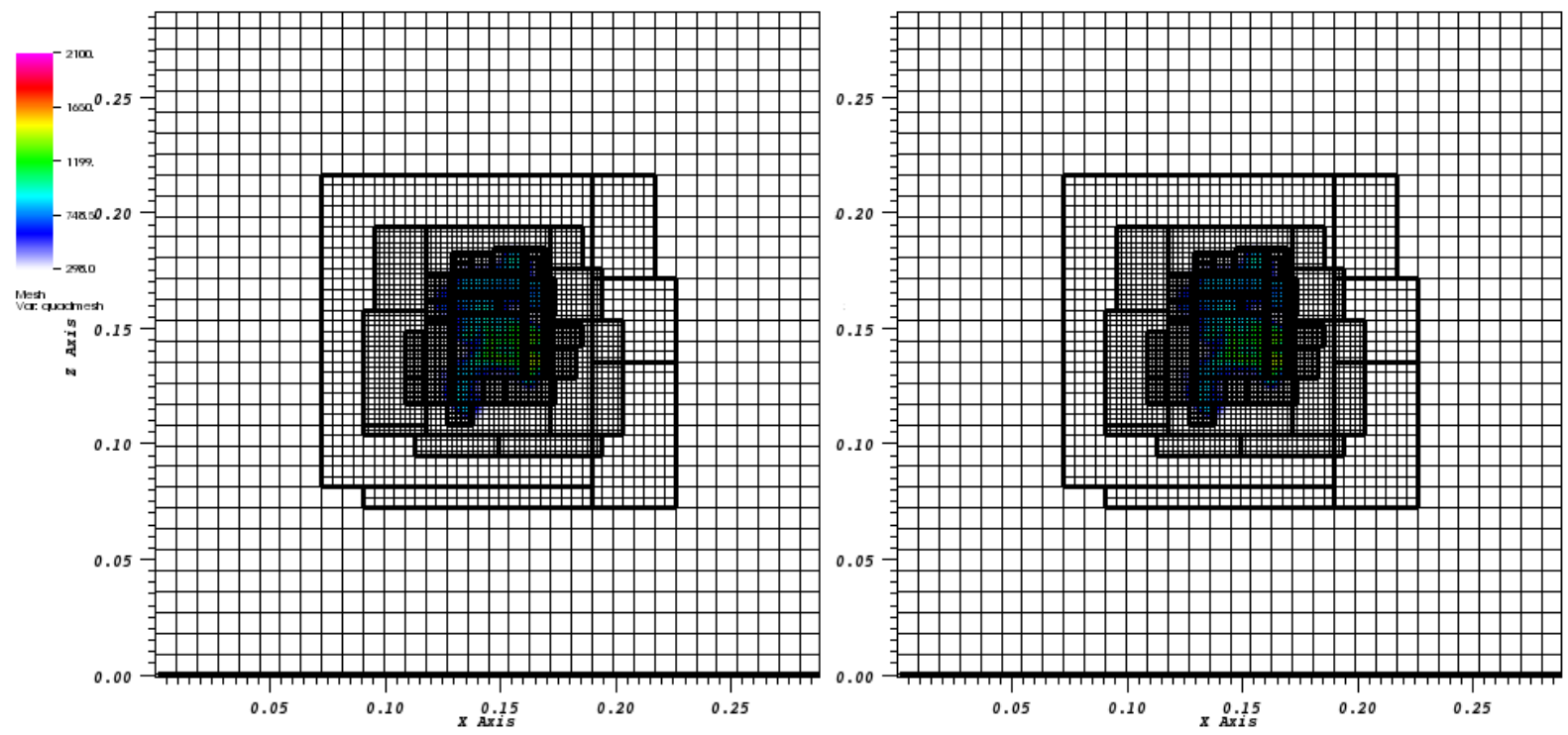

Figura 6.24: Temperatura do escoamento e malha bloco-estruturada gerada, em corte transversal na metade do domínio computacional.

O escalar conservado $z$ (fração de mistura) assume os seguintes valores na entrada do domínio computacional: $z=0$ onde o ar é injetado, $z=0.27$ na região piloto de diâmetro $d_{1}$ e $z=1$ onde o combustível é injetado, no bocal de diâmetro $d_{0}$. O valor estequiométrico do escalar conservado é $z_{e s t}=0.351$. As Figuras 6.25(a)-(f) mostram a evolução da fração de mistura do escoamento em um plano de corte no centro do domínio computacional. Note que a escala de cores varia do azul, indicando a presença do ar (oxidante) até o vermelho na região próxima do bocal, indicando a presença do combustível. A escala de cor intermediária indica a região onde ocorre a mistura entre combustível e oxidante e consequentemente a reação química. A temperatura do escoamento é obtida a partir da fração de mistura (veja a Seção 2.3). Note que o aumento de temperatura ocorre nas regiões onde combustível e oxidante se misturam (zona de reação). As Figuras 6.26(a)-(f) mostram a evolução da temperatura do escoamento.

As Figuras 6.27-6.29 mostram os perfis do decaimento da componente da velocidade na direção do escoamento e do escalar conservado, e o comportamento da temperatura, na linha de centro do domínio computacional. São apresentados dois perfis em cada figura, os quais possuem um mês de diferença de tempo de simulação computacional, mostrando a evolução do decaimento. Além disso, são apresentados os dados experimentais descritos nos trabalhos de Balow e Frank (2007) e Pitsch e Steiner (2000). É importante salientar que, para o tempo de simulação considerado, não é esperada a concordância entre os dados da simulação e os dados experimentais pois, para tais levantamentos estatísticos, os resultados deveriam começar a ser coletados após pelo menos um tempo de residência isto é, o tempo que uma partícula leva para cruzar completamente o domínio computacional. Pelas figuras, é possível notar que o jato atingiu pouco além da metade do domínio.

Os decaimentos apresentados nas Figuras 6.27-6.29 foram coletados bem antes do tempo de residência, devido ao tempo computacional exigido para realizar cada passo de integração da simulação numérica. Para o estágio do escoamento mostrado na Figura 6.26(f) o tempo computacional 


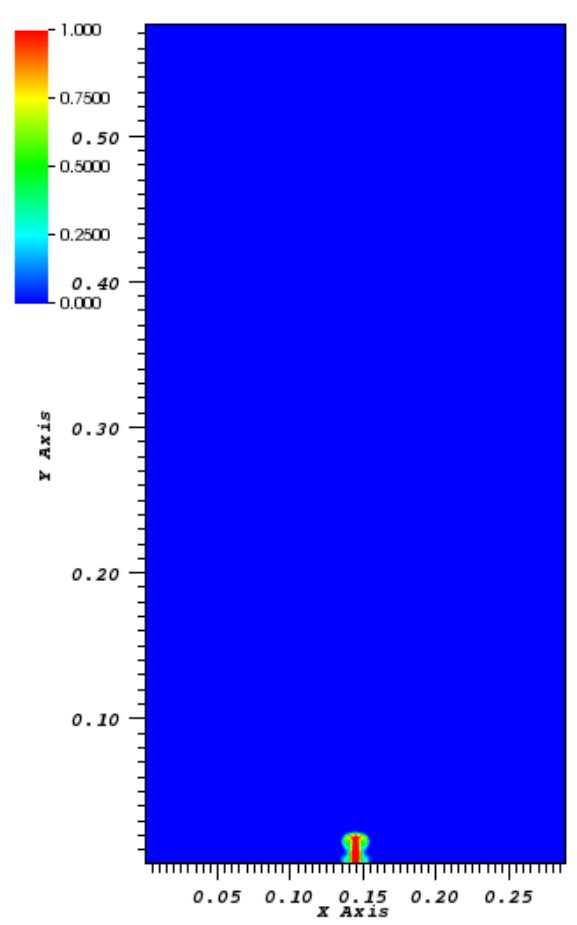

(a)

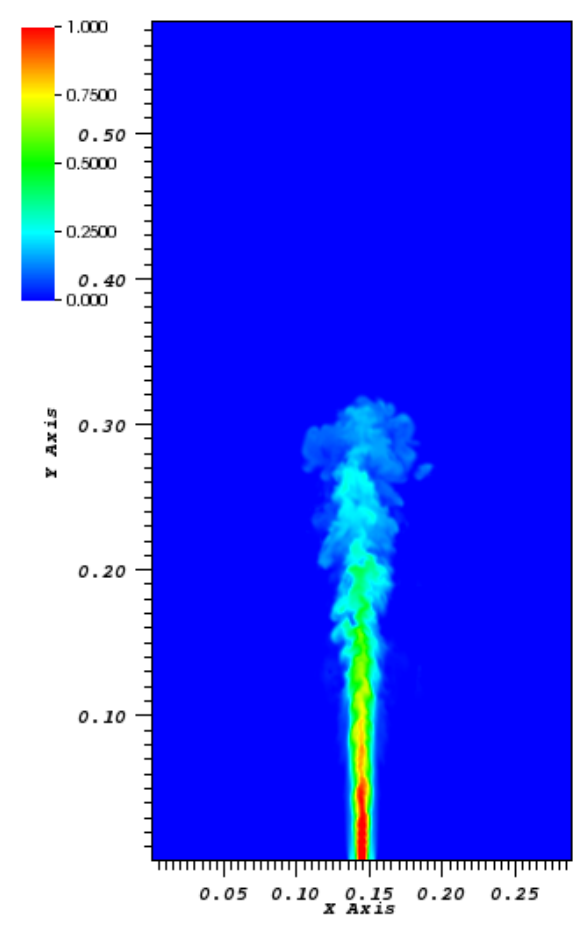

(d)

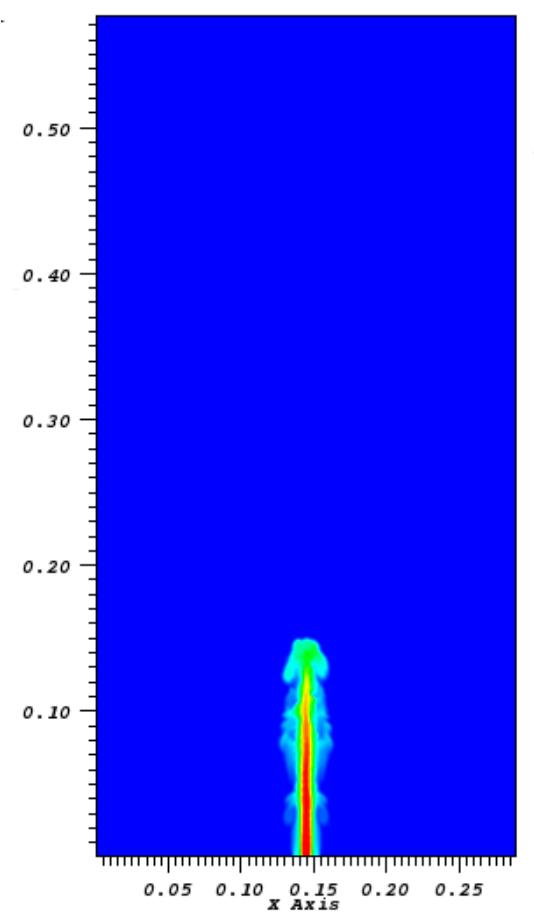

(b)

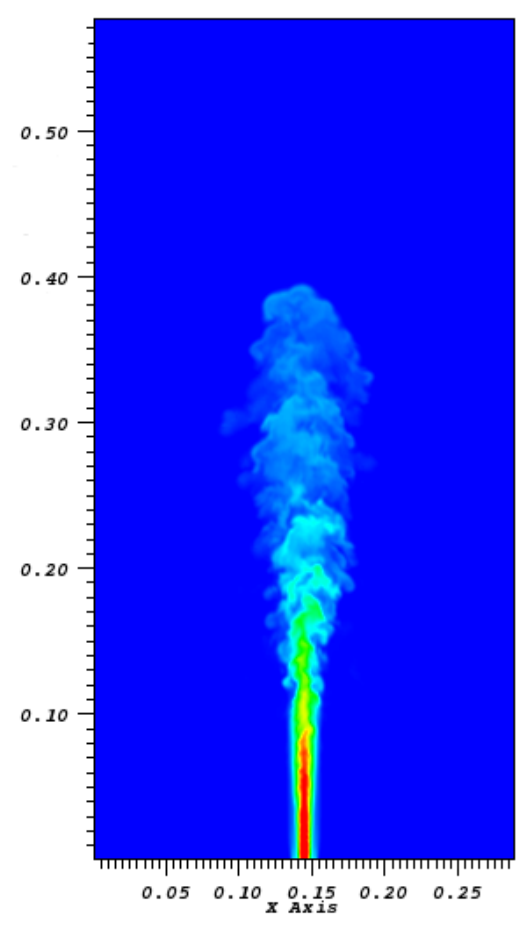

(e)

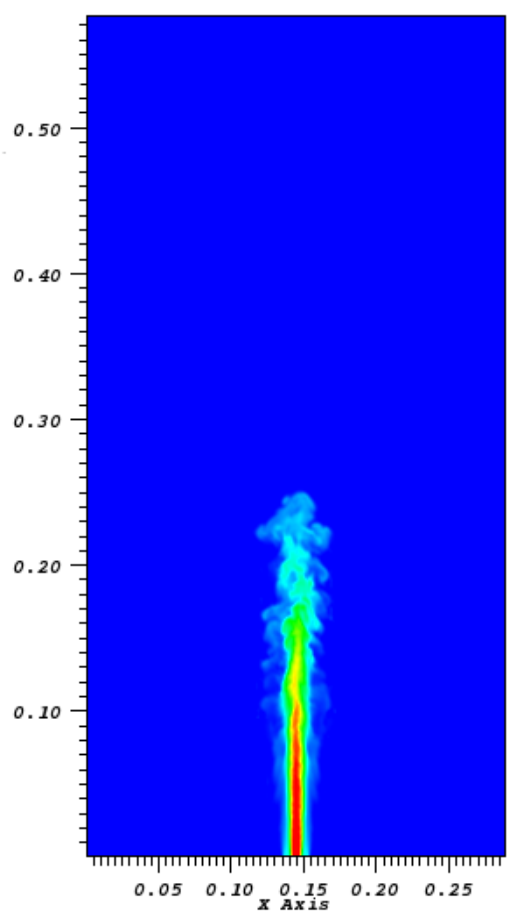

(c)

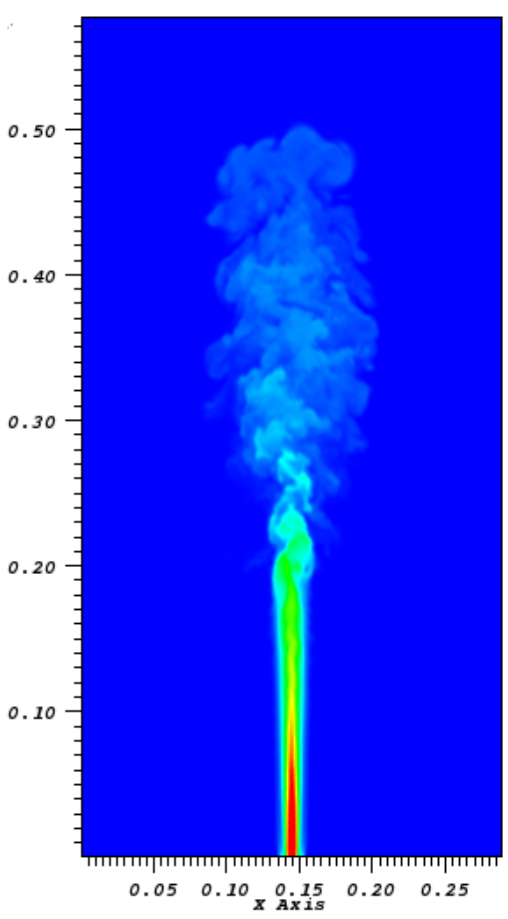

(f)

Figura 6.25: Escoamento reativo turbulento: Fração de mistura em um plano de corte no centro do domínio computacional em $x_{3}=1.44 \times 10^{-1} \mathrm{~m}$.

gasto por passo de integração, foi de aproximadamente $15 \mathrm{~min}$. O tempo computacional por passo de integração tende a aumentar já que o jato está se desenvolvendo e com isso a malha blocoestruturada adaptativa vai aumentando. Com base nas informações do jato incompressível turbulento (veja a Seção 6.1.2) serão necessárias pelo menos 13000 passos de integração para que o jato 


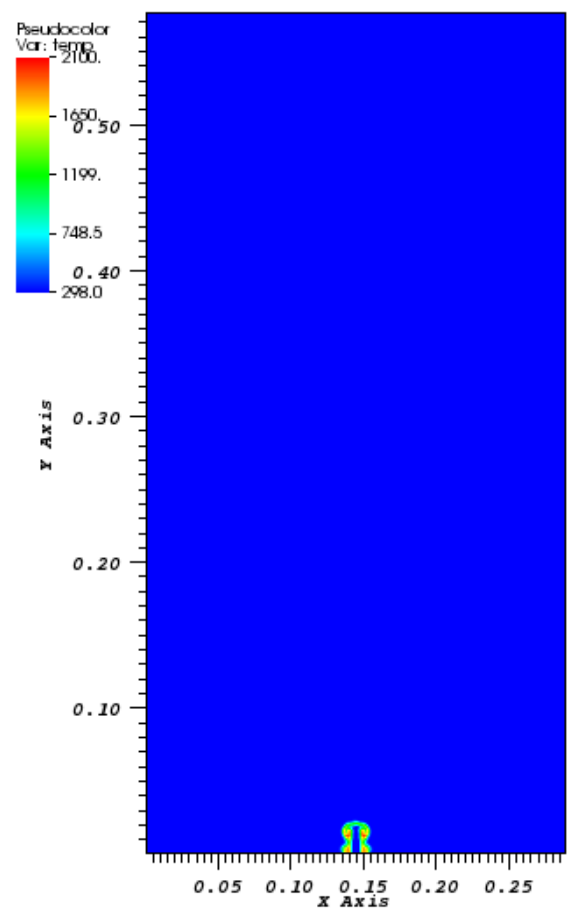

(a)

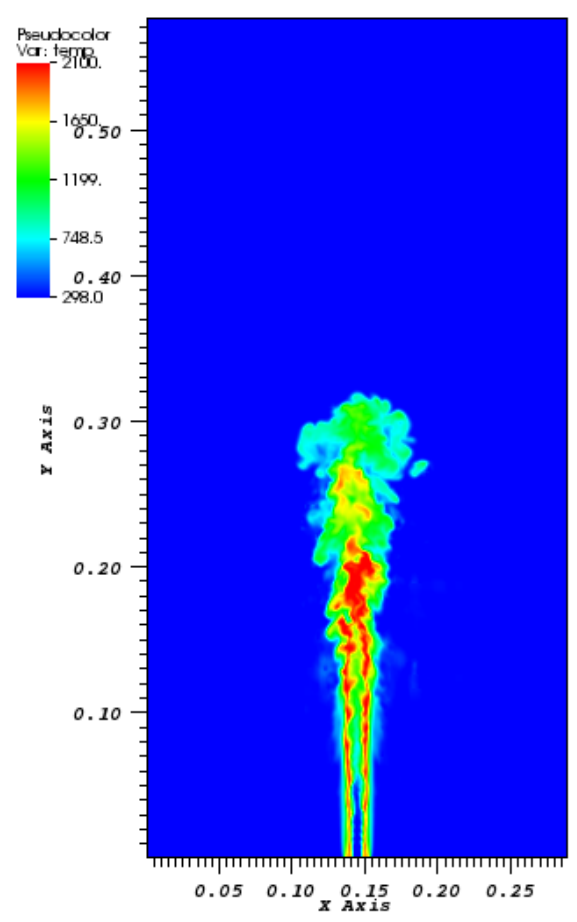

(e)

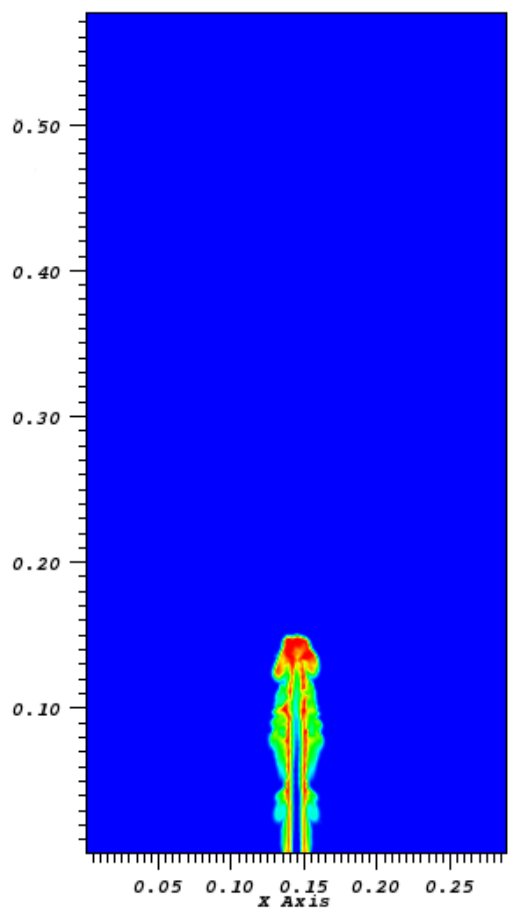

(b)

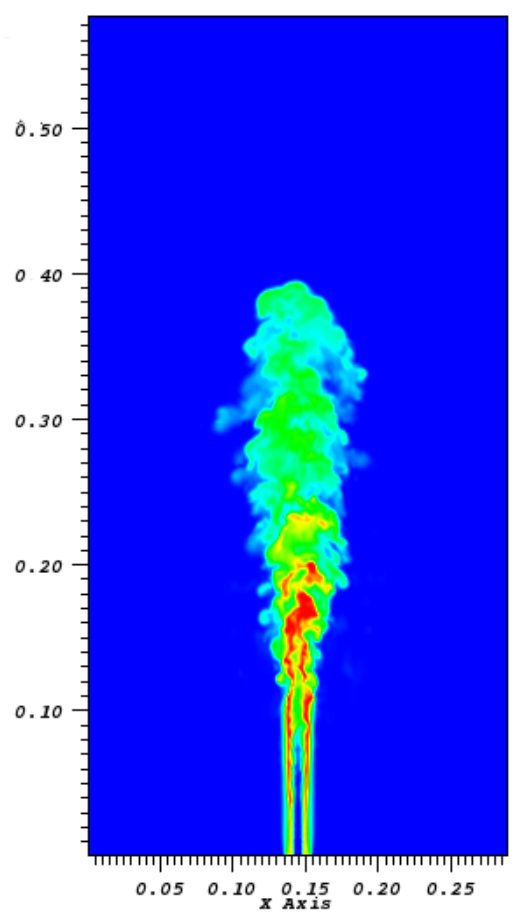

(d)

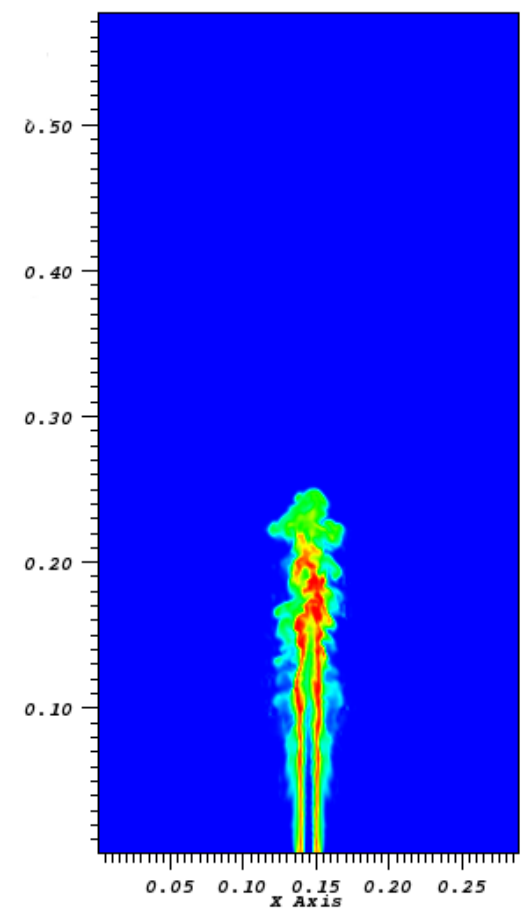

(c)

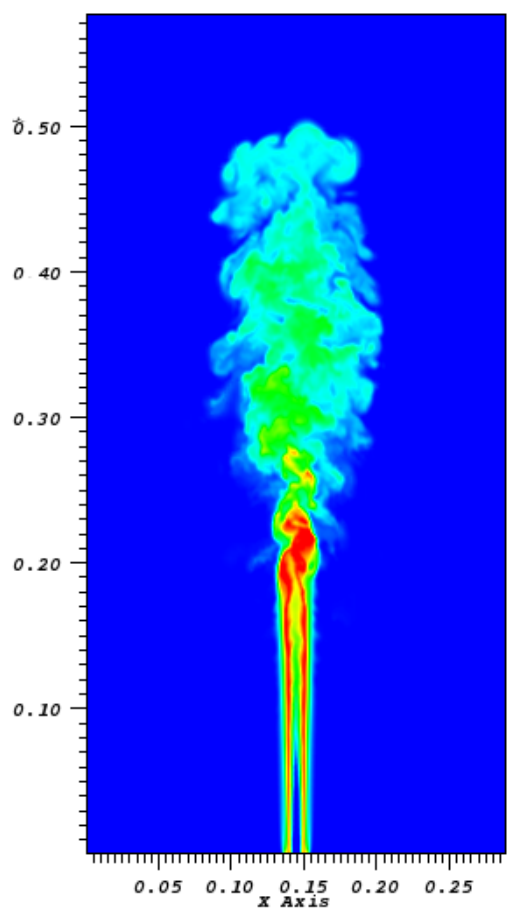

(f)

Figura 6.26: Escoamento reativo turbulento: Temperatura do escoamento em um plano de corte no centro do domínio computacional em $x_{3}=1.44 \times 10^{-1} \mathrm{~m}$.

complete um tempo de residência. 


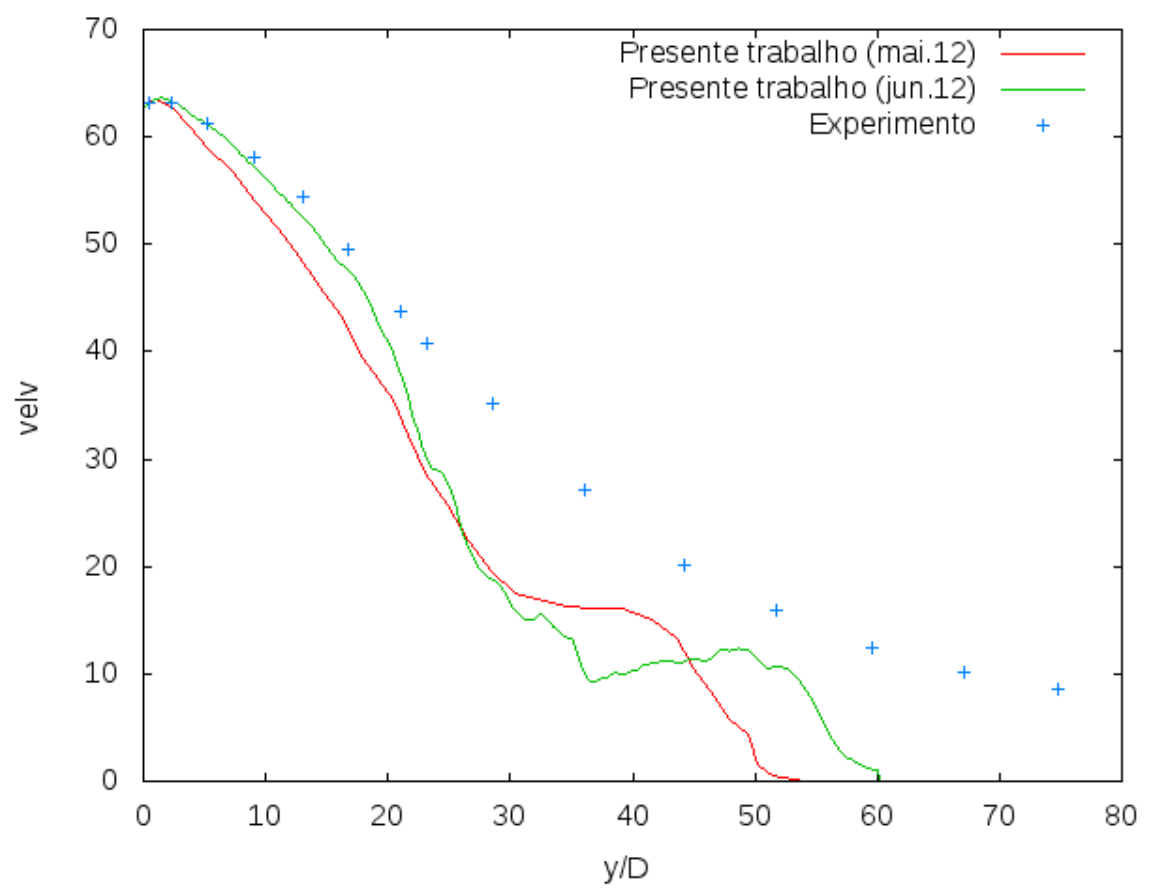

Figura 6.27: Decaimento na linha de centro da velocidade média na direção do escoamento e dados experimentais.

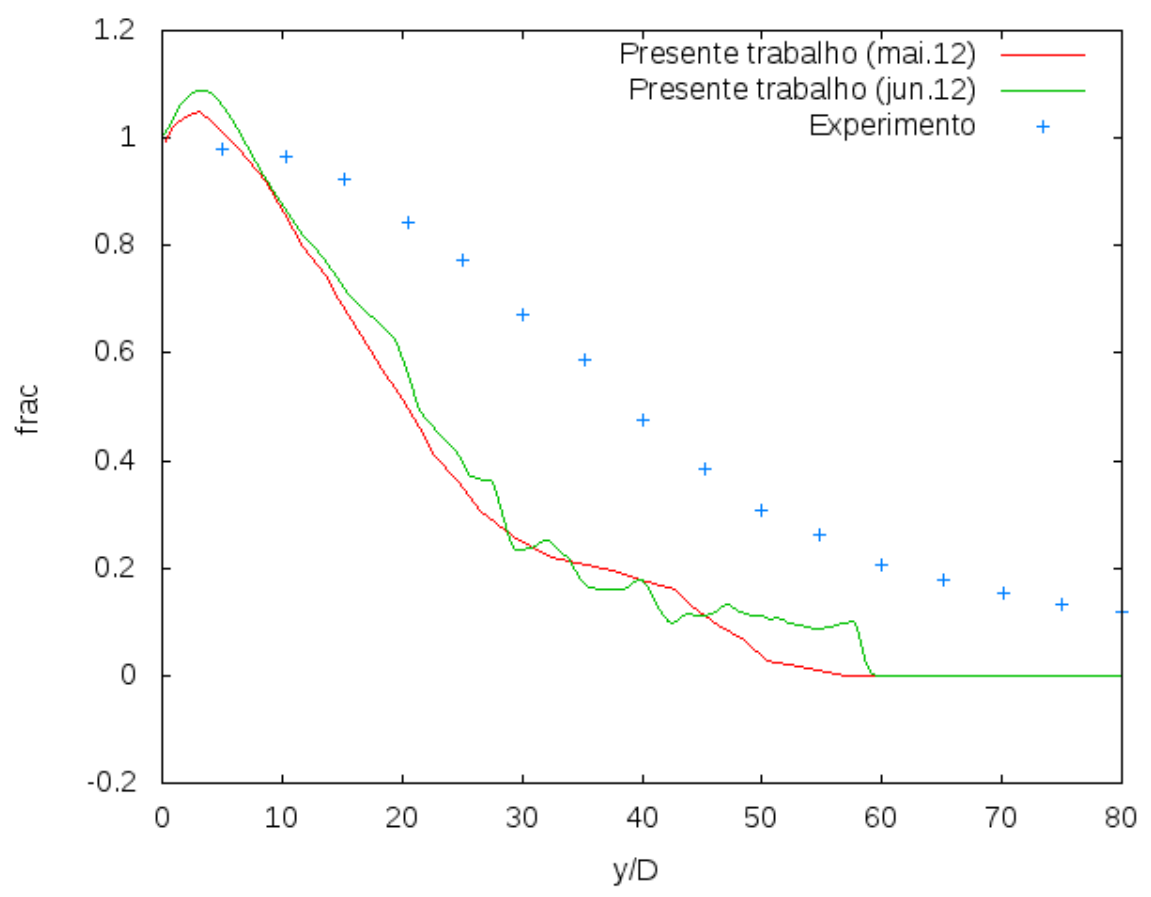

Figura 6.28: Decaimento na linha de centro na direção do escoamento da fração demistura média e dados experimentais.

\subsection{Sumário do presente capítulo}

A dificuldade das simulações numéricas descritas é ainda maior pois, além do tempo de processamento requerido, há que se varrer uma quantidade enorme de parâmetros na simulação numérica para ajustar de maneira apropriada o modelo matemático e as condições de simulação. Dentre tais parâmetros, são particularmente notáveis: 


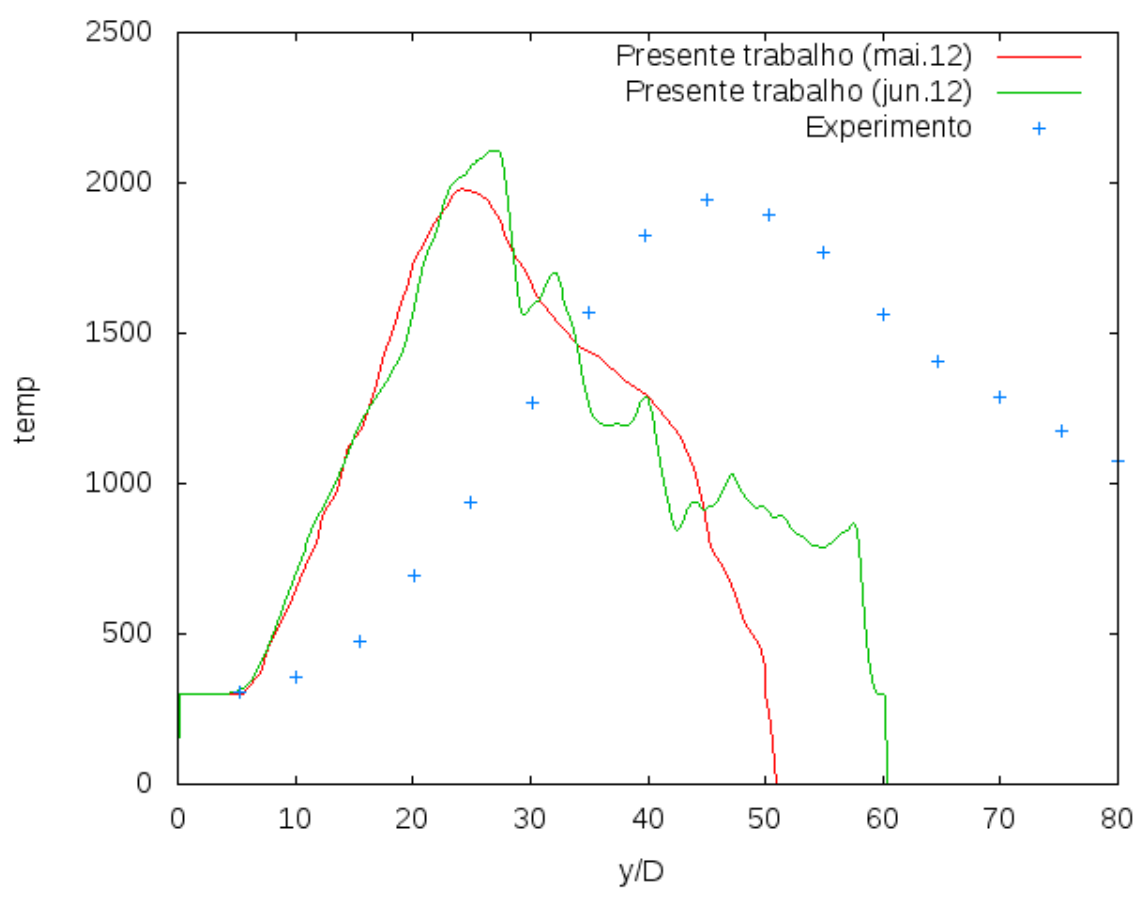

Figura 6.29: Perfil da temperatura na linha de centro na direção do escoamento e dados experimentais.

- Os parâmetros computacionais: o número de níveis de refinamento, os critérios de remalhagem, os critérios de convergência do métodos multigrid e o tamanho do passo de integração.

- Os parâmetros do modelo matemático: o formato e a contribuição relativa do ruído na condição de entrada e a constante de Smagorinsky do modelo de turbulência.

- Os parâmetros físicos: o tamanho do domínio computacional, as distribuições estatísticas que regem a injeção das gotículas.

Algumas tentativas foram realizadas considerando três e cinco níveis de refinamento. Três níveis de refinamento mostraram-se inadequados, pois a física do problema (como a turbulência) não foi bem capturada. Neste caso a região do bocal teria apenas 3 células computacionais na região do bocal. Na prática, isto é muito pouco, insuficiente para capturar o fenômeno. Boersma et al. (1998) descreve que doze células são, no mínimo, suficientes. De et al. (2011) descrevem que vinte e quatro células computacionais são, no mínimo, suficientes para escoamentos com gotículas. Isso implicaria em pelo menos cinco níveis de refinamento para as simulações numéricas sem gotículas e seis níveis de refinamento para a simulação com gotículas. Com cinco níveis de refinamento, os resultados numéricos melhoraram muito mas o código estendido AMR3D, sendo totalmente sequencial, provou-se inadequado para tal simulação numérica, pois o tamanho do passo torna-se 2.5 vezes menor do que o caso com quatro níveis de refinamento estimando-se no mínimo 6 meses de processamento.

Testando diferentes valores entre $[0.1,0.18]$ para a constante $C_{s}$ do modelo de turbulência de Smagorinsky, determinou-se que $C_{s}=0.15$ apresentou melhor decaimento da componente da velocidade na linha de centro. O mesmo pode-se descrever para a intensidade da turbulência na entrada do domínio computacional, para os resultados apresentados usou-se $2 \%$ de intensidade de ruído na componente da velocidade na direção do escoamento. Muitos trabalhos utilizam o 
modelo de Smagorinsky dinâmico (Apte et al. (2009), Pitsch e Steiner (2000), Pierce e Moin (2004) e Bini e Jones (2009)). Futuramente, pretende-se investir neste modelo de turbulência.

Para simular escoamentos reativos, considerando o tempo computacional gasto em um passo de integração do tempo, é necessário lembrar que o código estendido AMR3D é totalmente sequencial, característica herdada do código AMR3D original. Além disso, para obter segunda ordem de precisão, a cada passo de integração no tempo são resolvidas duas vezes cinco equações parábolicas e uma equação elíptica, totalizando doze equações a derivadas parciais sendo que as de natureza elíptica são mal condicionadas e tomam cerca de $80 \%$ do tempo de processamento por passo no tempo. Algumas tentativas de diminuir o tempo gasto a cada passo de integração foram tomadas:

- Aumento da constante CFL: a constante utilizada para a simulação do escoamento reativo na expressão (3.27) é $C=0.5$. Com seu aumento, aparece sinais de instabilidade numérica. A quantidade de iterações (V-ciclos) começa a aumentar aos poucos até o momento em que atinge o número máximo.

- Uso da norma $L_{2}$ no cálculo do tamanho de passo $\Delta t$ : a substituição da norma $L_{\infty}$ pela norma $L_{2}$, na expressão (3.27) gera instabilidades numéricas. O resíduo no método multigrid-multinível cai bem mais lentamente que o usual e acaba-se por atingir um número máximo de iterações (V-ciclos) em poucos passos de integração no tempo (qualquer que seja este limite adotado).

- Aumento da tolerância do Multigrid-multinível: relaxando o critério de convergência do método multigrid-multinível para $\mathrm{O}(\Delta x)$, onde $\Delta x$ é o espaçamento da malha mais fina, o número de iterações realizado pela equação elíptica diminuiu em média 3 V-ciclos dos 25 que vinham sendo realizados. Uma economia de aproximadamente 1.2\%. Diante da pequena diferença decidiu-se manter o critério de convergência de $\mathrm{O}\left(\Delta x^{2}\right)$ para garantir que a precisão espacial não fosse comprometida. Esta decisão foi feita com base nas verificações numéricas que não atingiam segunda ordem de aproximação caso a tolerância para o resíduo não fosse escolhida pequena o suficiente.

Mesmo com o uso da técnica de refinamento adaptativo de malhas, o código estendido AMR3D em sua atual implementação mostra-se por demais custoso para problemas onde o escoamento reativo encontra-se no limiar de se desenvolver, quando muitos blocos de refinamento são gerados. O perfil do código para esta simulação identificou que o maior consumo de tempo é gasto na resolução da equação da correção da pressão, com aproximadamente $80 \%$ do tempo gasto em um passo de integração. As subrotinas mais requisitadas são responsáveis pelo preenchimento de células fantasmas as quais, juntas, consomem aproximadamente $91 \%$ do tempo gasto em um passo de integração do tempo, havendo 2 bilhões de chamadas desta subrotina em um único passo de integração. A estratégia de diminuir o número de blocos discretizados na região principal do escoamento foi mantida (o uso de "tubo" central) diminuindo em $30 \%$ o tempo gasto pelo conjunto de subrotinas responsáveis pelo preenchimento de células fantasmas. Além de investigar outras estratégias para diminuir o número de blocos discretizados, o caminho natural para resolver o principal "gargalo" do código estendido AMR3D, seria resolver pelo menos, a equação da correção da pressão (a equação elíptica) em computação paralela. 


\subsection{Nomenclatura do presente capítulo}

$d_{0} \quad$ Diâmetro do bocal de injeção de combustível $(m)$

$d_{1} \quad$ Diâmetro do bocal piloto $(m)$

$d_{p} \quad$ Diâmetro da gotícula $p(m)$

$D \quad$ Coeficiente de difusão de massa $\left(\mathrm{m}^{2} / \mathrm{s}\right)$

$N_{p} \quad$ Número de gotículas

$p \quad$ Pressão $(\mathrm{atm})$

$p_{0} \quad$ Pressão termodinâmica $(\mathrm{atm})$

$p_{2} \quad$ Pressão dinâmica $(\mathrm{atm})$

$r_{0} \quad$ Raio do bocal de injeção de combustível $(m)$

$r_{1} \quad$ Raio do bocal piloto $(m)$

$t \quad$ tempo $(s)$

$T \quad$ Temperatura $(K)$

$u_{2} \quad$ Componente da velocidade na direção do escoamento $(\mathrm{m} / \mathrm{s})$

$u_{2, d_{0}}$ Componente da velocidade na direção do escoamento na entrada do combustível $(\mathrm{m} / \mathrm{s})$

$\mathbf{u} \quad$ Velocidade do escoamento $(\mathrm{m} / \mathrm{s})$

$\mathbf{U}_{p} \quad$ Velocidade da gotícula $(\mathrm{m} / \mathrm{s})$

$\mathbf{X}_{p} \quad$ Posição de uma gotícula $(m)$

$Y \quad$ Fração mássica de uma espécie química

$z \quad$ Escalar conservado normalizado

\section{Letras Gregas}

$\kappa \quad$ Condutividade térmica $(\mathrm{kg} / \mathrm{ms})$

$\mu \quad$ Viscosidade dinâmica $\left(N \times s / m^{2}\right)$

$\rho \quad$ Massa específica, densidade $\left(\mathrm{kg} / \mathrm{m}^{3}\right)$

\section{Adimensionais}

$R e_{r} \quad$ Número de Reynolds (raio) Re Número de Reynolds

$\operatorname{Pr} \quad$ Número de Prandtl Le Número de Lewis 


\section{Capítulo 7}

\section{Considerações finais}

O presente trabalho estende de maneira original uma metodologia numérica anterior cujo foco eram os escoamentos incompressíveis. A extensão proposta é inédita e é aplicável à simulação computacional de escoamentos reativos a baixo número de Mach (combustão computacional). Tal proposta parte de três componentes principais: uma técnica de refinamento adaptativo de malhas para discretizar o domínio computacional, a utilização de esquemas implícitos-explícitos para a discretização no tempo das equações da dinâmica do escoamento e o Método da Projeção em variáveis primitivas para tratar o acoplamento pressão-velocidade. Embora formada por componentes individualmente conhecidas, a combinação de tais elementos é nova e resulta numa metodologia para abordar escoamentos a baixo número de Mach cujo uso e potencial são aqui cuidadosamente investigados.

A ideia é agregar ao modelo matemático inicialmente considerado e de onde se partiu, isto é, as equações de Navier-Stokes para um escoamento incompressível transiente com propriedades materiais variáveis, um novo conjunto de equações que modelam novas propriedades do fluido: as equações de conservação de massa, das concentrações das espécies químicas e de energia. Basicamente, empregando a abordagem dada por fração de mistura para descrever as concentrações de combustível e oxidante chega-se a um modelo matemático simplificado o qual é tratado pela primeira vez empregando-se malhas adaptativas bloco-estruturadas e o Método da Projeção. É preciso salientar que o método da Projeção, no contexto da extensão proposta, está baseado na imposição de um divergente não nulo para o campo de velocidade, oriunda da combinação de uma equação termodinâmica de estado com a equação de conservação de massa. A hipótese do número de Mach ser baixo é usada na dedução do modelo matemático aqui abordado. Para tratar escoamentos turbulentos, emprega-se a metodologia LES com o modelo de turbulência de Smagorinsky.

O código computacional oriundo das idéias anteriores, empregado nas simulações computacionais de escoamentos com baixo número de Mach, é novo e representa uma das contribuições do presente trabalho. Tal código teve por base o código computacional denominado "AMR3D", escrito em Fortran90, cujo desenvolvimento tem sido feito num esforço conjunto e contínuo do IME-USP e do MFLab/FEMEC-UFU, por vários anos. Os métodos numéricos empregados na resolução das novas equações de advecção-difusão discretizadas (e.g. para a fração de mistura e para a energia) baseiam-se em métodos multigrid-multinível. Uma abordagem que, até recentemente, foi considerada natural no contexto de malhas adaptativas bloco-estruturadas (mais comentários sobre este assunto são feitos mais adiante visando à continuidade deste trabalho). Para tratar instabilidades numéricas oriundas da discretização da equação de conservação de massa (uma equação de caráter 
hiperbólico), discretiza-se o termo de advecção empregando-se o método QUICK, além de se incluir um termo de difusão artificial, um procedimento comumente adotado.

Efetuou-se uma bateria inicial de testes abordando casos de escoamentos incompressíveis nos regimes laminar e turbulento. Tipicamente, estes casos tratados permitem a identificação preliminar de forma mais rápida dos pontos da metodologia que devem receber atenção especial e ser otimizados (eventualmente reprogramados). Para tanto, empregou-se o código AMR3D, especialmente preparado para, dentre outros, estudos de otimização da parte responsável pela geração de malhas adaptativas. É preciso salientar que tais simulações computacionais foram executadas pela primeira vez no contexto do AMR3D.

Uma das primeiras alterações foi armazenar os arquivos de visualização no formato Silo. Silo é um formato de dados e uma biblioteca com interface para a linguagem Fortran90 e C projetada para ler e escrever dados científicos. A bilioteca Silo suporta malhas bloco-estruturadas e o uso do HDF5 (Hierarchical Data Format 5). O HDF5 é um formato de arquivos muito usado para armazenar e gerenciar dados. Esta medida reduziu o tamanho dos arquivos em aproximadamente 15\%. Novos critérios de refinamento foram implementados, baseados na norma do tensor de tensões viscosas, na vorticidade e na norma da turbulência. A calibração destes critérios de refinamento foi feita cuidadosamente. Testes para a escolha da constante do modelo de Smagorinsky também foram realizados. Com a simulação de um jato turbulento foram identificadas informações relevantes associadas à metodologia numérica. Dentre elas, o uso abusivo de células fantasmas que levam a um alto custo computacional. O número de células fantasmas está associado ao número de blocos discretizados gerados pela estratégia AMR. A solução foi diminuir o número de blocos discretizados (estratégia do "tubo"). Esta simples medida resultou em um aumento de eficiência de quase $30 \%$. Além disso, foram realizados testes com diferentes compiladores. Apesar das medidas tomadas, algumas simulações chegaram a levar 12 semanas com passos no tempo de até 15 minutos.

Aos testes anteriores, seguiram testes de verificação e validação das equações de advecçãodifusão de grandezas escalares (incluindo a implementação do método multigrid-multinível, empregado na resolução dos sistemas lineares resultantes da discretização temporal e espacial) os quais comprovaram segunda ordem para estas grandezas. Foram realizados então testes para a conservação de massa. De natureza hiperbólica, estas equações demandam cuidados para sua estabilização. A utilização do esquema de diferenças finitas centradas (com o termo de difusão numérica) resultou no decaimento da ordem de convergência, como relatado na Seção 3.2.4. A substituição pelo esquema numérico QUICK resolveu o problema e manteve segunda ordem de aproximação.

A metodologia numérica para um escoamento incompressível (não-reativo) turbulento contendo gotículas inertes (sem evaporação) foi devidamente verificada para o modelo matemático da fase contínua e da fase dispersa. Alguns testes preliminares envolvendo a fase dispersa foram realizados. Estes testes tiveram como objetivo apontar as primeiras dificuldades a serem enfrentadas dentro do contexto AMR3D, ao se empregar uma nuvem de gotículas lagrangianas acompanhadas em sua trajetória. Para esta simulação foi acrescentado um critério de refinamento baseado na posição das gotículas lagrangianas. O critério de refinamento baseado nas gotículas está implementado de forma que uma célula computacional não seja remarcada na lista de pontos para refinamento. Isto porque uma mesma célula computacional pode conter mais que uma gotícula. A estrutura de dados (uma lista encadeada) utilizada para armazenar as gotículas facilita a remoção e a inserção 
das mesmas. Serão necessários testes com outras distribuições estatísticas para o diâmetro das gotículas para verificar a representatividade dos dados.

A metodologia numérica introduzida para um escoamento com baixo número de Mach foi cuidadosamente verificada por intermédio da estratégia de soluções manufaturadas. Foram abordados preliminarmente alguns problemas de aplicação: escoamentos reativos laminar e turbulento (em particular, no último caso, a Chama de difusão "Chama D"). A simulação de escoamentos reativos a baixo número de Mach incluem a solução de mais duas equações a cada passo de integração. Com isso, surge a dificuldade na escolha da combinação apropriada de parâmetros da simulação. Aqui incluem-se parâmetros computacionais (critérios de refinamento e número de níveis físicos), parâmetros físicos (perturbação na entrada do escoamento) e parâmetros dos modelos envolvidos (constante do modelo de turbulência). Estes parâmetros devem ser escolhidos cuidadosamente por meio de testes numéricos. Um código sequencial como o AMR3D não permite uma ampla bateria de testes. Além disso, a comparação dos resultados numéricos com os resultados experimentais é feita por meio do decaimento da velocidade na linha de centro. A análise deste decaimento deve ser feita após o escoamento estar desenvolvido. O principal "gargalo" do código a ser atacado será a resolução do sistema linear associado a equação da correção da pressão em paralelo. Um trabalho que está sendo desenvolvido pelo grupo IME-MFLab.

\subsection{Contribuições}

Originalmente, o código AMR3D utiliza a técnica AMR e o método da Projeção de ChorinTemann para escoamentos incompressíveis. A proposta de extensão da metodologia numérica para escoamentos reativos com baixo número de Mach, amplia a aplicabilidade da ferramenta a novos problemas de aplicação.

No cenário nacional o presente trabalho contribuiu para o desenvolvimento de um algoritmo adaptativo para a simulação tridimensional de escoamentos reativos com baixo número de Mach. A metodologia numérica associada ao modelo de reação química simplificado tem seu grau de originalidade. A inclusão da técnica de refinamento adaptativo de malhas, neste contexto, mostrouse eficaz e vantajosa.

A aplicação da metodologia numérica proposta a escoamentos incompressíveis com gotículas inertes, até onde se sabe, tem seu grau de originalidade. As simulações computacionais preliminares de escoamentos incompressíveis com gotículas inertes mostra que o uso da técnica AMR é promissor.

Esta foi a primeira vez que uma simulação de jato foi feita pelo grupo IME-MFLab no contexto do código AMR3D. Estas simulações de jatos incompressíveis (não-reativos) e de jatos reativos com baixo número de Mach serviram para mostrar as vantagens e algumas dificuldades da metodologia numérica adaptativa. Dentre as vantagens destacamos o tempo computacional ganho que ainda pode ser melhorado evitando-se o uso abusivo de células fantasmas. Com a simples ideia, de se aproveitar a estrutura do problema com a estratégia do tubo, houve um ganho significativo de aproximadamente $30 \%$ no tempo computacional. 


\subsection{Continuidade}

Durante a realização do presente trabalho surgiram algumas dificuldades que tornaram-se tópicos para a continuidade do mesmo. Dentre eles, destacam-se:

1. Investigação da simulação do problema via resolução matricial do sistema linear associado ao problema. Isso facilitaria a resolução do sistema com a utilização de bibliotecas, como a PETSc, que inclui métodos numéricos já paralelizados. Além disso, a inclusão da metodologia numérica proposta em uma versão paralela do código AMR3D.

2. Investigação e implementação de esquemas numéricos conservativos e de alta ordem para a aproximação dos termos advectivos. Com isso pretende-se retirar o termo de difusão numérica na equação da conservação da massa.

3. Simulação da chama de difusão com parâmetros de simulação "adequados".

4. Desenvolvimento em combustão computacional com ênfase em sprays inertes com e sem modelagem de evaporação.

5. Implementação de estruturas de dados eficientes para a localização das gotículas em diferentes níveis de refinamento na malha bloco-estruturada.

6. Investigação do efeito da turbulência no transporte das gotículas. Implementação do modelo de turbulência de Smagorinsky dinâmico. Implementação de outras formas de simular a turbulência na entrada do domínio computacional.

7. Investigação da necessidade de manter as gotículas lagrangianas no nível mais fino de refinamento.

8. Aplicação da metodologia proposta junto com a metodologia da fronteira imersa para simular escoamentos reativos em geometrias complexas, por exemplo uma câmara de combustão. 


\section{Apêndice A}

\section{Modelo de turbulência}

O fenômeno da turbulência é encontrado em muitos processos de combustão, como foguetes, fornos e queimadores industriais. A turbulência ocorre em escoamentos com altos número de Reynolds. Assumindo-se que as propriedades do escoamento podem ser decompostas em uma parte filtrada e uma parte dinâmica, também chamada flutuação como descrevem Versteeg e Malalasekera (2007), Poinsot e Veynante (2005), Turns (1996) e Pope (2000). Esta decomposição é chamada de Decomposição de Reynolds e é dada por

$$
q=\tilde{q}+q^{\prime}
$$

onde $\tilde{q}$ representa a parte filtrada e $q^{\prime}$ representa uma flutuação.

Os métodos numéricos para descrever a turbulência podem ser agrupados em três categorias: as equações médias de Reynolds (RANS - Reynolds Average Navier-Stokes), a simulação das grandes escalas (LES - Large Eddy Simulation) e a simulação numérica direta (DNS - Direct Numerical Simulation). Os modelos de turbulência para as equações médias RANS foram desenvolvidos para resolver apenas os valores médios de todas as propriedades nas equações de Navier-Stokes. As equações de balanço das quantidades médias são obtidas a partir das equações de balanço instântaneas quando substituimos a expressão (A.1) nas equações descritas no Capítulo 2. As equações médias requerem modelos de fechamento, como o modelo $k-\epsilon$, para os termos extras que surgem das interações não lineares da turbulência. Na simulação numérica direta, as equações de Navier-Stokes instântaneas são resolvidas completamente sem qualquer modelo de turbulência. Todas as escalas de turbulência são explicitamente determinadas e seus efeitos capturados. A simulação numérica direta possui alto custo computacional pois é necessária uma malha muito fina para capturar todos os efeitos da turbulência, veja Pope (2000). Na aproximação LES as grandes escalas turbulentas são explicitamente calculadas ao passo que os efeitos das menores escalas são incluídos usando modelos de submalha conforme descrevem Versteeg e Malalasekera (2007), Poinsot e Veynante (2005) e Pope (2000).

No presente trabalho foi utilizada a aproximação LES. As equações de conservação para esse modelo de turbulência são obtidas pela filtragem das equações de conservação instantâneas descritas no Capítulo 2. Os valores filtrados das propriedades são obtidos por meio da expressão

$$
\widetilde{q}=\int q\left(\mathbf{x}^{\prime}\right) F\left(\mathbf{x}-\mathbf{x}^{\prime}\right) d \mathbf{x}^{\prime}
$$


onde $F$ é o filtro do modelo da turbulência. Por exemplo, o filtro caixa é descrito por

$$
F(\mathbf{x})=F\left(x_{1}, x_{2}, x_{3}\right)=\left\{\begin{array}{l}
\frac{1}{\Delta^{3}}, \text { se }\left|x_{i}\right| \leq \frac{\Delta}{2} \text { para } 1 \leq i \leq 3 \text { ou } \\
0, \text { caso contrário }
\end{array}\right.
$$

onde $\Delta^{3}=\Delta x_{1} \Delta x_{2} \Delta x_{3}$. O filtro caixa corresponde a média sobre o cubo (célula computacional) com dimensões $\Delta x_{1}, \Delta x_{2}$ e $\Delta x_{3}$. Os filtros são normalizados, ou seja,

$$
\int_{-\infty}^{\infty} \int_{-\infty}^{\infty} \int_{-\infty}^{\infty} F\left(x_{1}, x_{2}, x_{3}\right) d x_{1} d x_{2} d x_{3}=1
$$

Para mais exemplos de filtros veja Pope (2000) e Poinsot e Veynante (2005).

Para os problemas com densidade variável costuma-se utilizar o filtro de Favre,

$$
\widetilde{q}(x)=\frac{1}{\bar{\rho}} \int \rho q\left(\mathbf{x}^{\prime}\right) F\left(\mathbf{x}-\mathbf{x}^{\prime}\right) d \mathbf{x}^{\prime} .
$$

A quantidade filtrada $\widetilde{q}$ é calculada numericamente ao passo que a perturbação $q^{\prime}=q-\widetilde{q}$ corresponde a parte não resolvida. As equações de conservação filtradas para o método LES são descritas por

$$
\begin{aligned}
\frac{\partial \bar{\rho}}{\partial t}+\frac{\partial\left(\bar{\rho} \widetilde{u}_{j}\right)}{\partial x_{j}} & =0, \\
\frac{\partial \bar{\rho} \widetilde{u}_{i}}{\partial t}+\frac{\partial\left(\bar{\rho} \widetilde{u}_{j} \widetilde{u}_{i}\right)}{\partial x_{j}} & =-\frac{\partial \bar{p}}{\partial x_{i}}+\frac{\partial}{\partial x_{j}}\left(\widetilde{\tau}_{j i}+t_{j i}\right), \text { com } i=1,2,3 \mathrm{e} \\
\frac{\partial \bar{\rho} \widetilde{z}}{\partial t}+\frac{\partial\left(\bar{\rho} \widetilde{u}_{j} \widetilde{z}\right)}{\partial x_{j}} & =\frac{\partial}{\partial x_{j}}\left(\bar{\rho} \widetilde{D} \frac{\partial \widetilde{z}}{\partial x_{j}}+\bar{\rho} q_{z}\right) .
\end{aligned}
$$

As equações filtradas (A.6)-(A.8) necessitam de modelos de fechamento para os termos $t_{j i}$ e $\bar{\rho} q_{z}$. O termo $t_{i j}=\left(\widetilde{u_{i} u_{j}}-\widetilde{u}_{i} \widetilde{u}_{j}\right)$ é modelado com a hipótese da viscosidade turbulenta conforme Pope (2000), Versteeg e Malalasekera (2007), Poinsot e Veynante (2005) e Pierce e Moin (2004), dada por

$$
t_{i j}-\frac{\delta_{i j}}{3} t_{k k}=-\mu_{t}\left(\frac{\partial \widetilde{u_{i}}}{\partial x_{j}}+\frac{\partial \widetilde{u_{j}}}{\partial x_{i}}-\frac{2}{3} \delta_{i j} \frac{\partial \widetilde{u_{k}}}{\partial x_{k}}\right)=-2 \mu_{t}\left(\widetilde{S_{i j}}-\frac{\delta_{i j}}{3} \widetilde{S_{k k}}\right)
$$

sendo $\mu_{t}$ a viscosidade turbulenta dada pelo modelo de Smagorinsky,

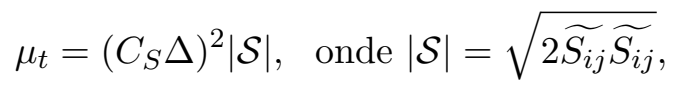

onde $|\mathcal{S}|$ é o módulo da taxa de deformação filtrada, $\Delta$ é o tamanho do filtro e $C_{S}$ é uma constante do modelo, calculada de maneira dinâmica (veja Germano et al. (1991) e Pitsch (2006)) ou escolhida constante dependendo do tipo de escoamento.

O termo $q_{z}$ é modelado com a hipótese do gradiente da difusão descrita por Pierce e Moin (2004), dada por

$$
q_{z}=-\bar{\rho}\left(\widetilde{u_{i} z}-\widetilde{u}_{i} \widetilde{z}\right)=-\bar{\rho} D_{t} \frac{\partial \widetilde{z}}{\partial x_{i}}
$$

onde a difusividade turbulenta é dada por

$$
D_{t}=\left(C_{z} \Delta\right)^{2}\left|\mathcal{S}_{i j}\right| .
$$


A diferença entre a difusividade turbulenta e a viscosidade turbulenta, nas equações (A.10) e (A.12), respectivamente, está nos coeficientes $C_{S}$ e $C_{z}$ destas equações. A razão entre estes coeficientes fornece o número de Schimdt turbulento, dado por

$$
S c_{t}=\frac{C_{S}}{C_{z}}
$$

Desta forma, as equações filtradas (A.7) e (A.8) com os respectivos modelos de turbulência são dadas por

$$
\bar{\rho}\left(\frac{\partial \widetilde{u}_{i}}{\partial t}+\widetilde{u}_{i} \frac{\partial \widetilde{u}_{j}}{\partial x_{j}}\right)=-\frac{\partial \widetilde{p}}{\partial x_{i}}+\frac{\partial}{\partial x_{j}}\left[\mu\left(\frac{\partial \widetilde{u}_{j}}{\partial x_{i}}+\frac{\partial \widetilde{u}_{i}}{\partial x_{j}}\right)-\frac{2}{3} \delta_{i j} \frac{\partial \widetilde{u_{k}}}{\partial x_{k}}\right]
$$

sendo $\mu=\mu_{c}+\mu_{t} \mathrm{e}$

$$
\frac{\partial(\bar{\rho} \widetilde{z})}{\partial t}+\frac{\partial\left(\bar{\rho} \widetilde{u}_{i} \widetilde{z}\right)}{\partial x_{i}}=\frac{\partial}{\partial x_{i}}\left(D \frac{\partial \widetilde{z}}{\partial x_{i}}\right)
$$

sendo $D=\bar{\rho} \widetilde{D}+D_{t}$.

\section{A.1 Modelo de turbulência para a taxa de reação}

Conforme comentado na Seção 2.1 uma dificuldade ligada ao termo $\dot{\omega}_{l}$, o qual determina a taxa de produção ou de consumo da espécie química $l$, está na modelagem da turbulência. O termo $\dot{\omega}_{C}$, para uma reação de um único passo descrita na Seção 2.3 , é dado por

$$
\dot{\omega}_{C}=-A \rho^{2} T^{\alpha} Y_{C} Y_{O} \exp \left(\frac{-T_{a}}{T}\right) .
$$

Como a taxa de reação, na expressão (A.16) é não linear, modelar os termos $\dot{\omega}_{C}$ não é simplesmente expressá-lo como uma função das quantidades filtradas $\widetilde{Y}_{C}, \widetilde{Y}_{O}$ e $\widetilde{T}$. O termo $\widetilde{\omega}_{C}$ é obtido por meio de uma expansão em série de Taylor, conforme Versteeg e Malalasekera (2007), Poinsot e Veynante (2005) e Veynante e Vervisch (2002). Após filtrar a temperatura, $T=\widetilde{T}+T^{\prime \prime}$, dada uma função $f(T)$ tem-se que $f(T)=f\left(\widetilde{T}+T^{\prime \prime}\right)$. Expandindo $f(T)$ em série de Taylor obtém-se

$$
f(T)=f\left(\widetilde{T}+T^{\prime \prime}\right)=f(\widetilde{T})+T^{\prime \prime} f_{\widetilde{T}}(\widetilde{T})+\frac{T^{\prime \prime 2}}{2} f_{\widetilde{T} \widetilde{T}}(\widetilde{T})+O\left(T^{\prime \prime 3}\right),
$$

sendo $f_{\widetilde{T}}=\frac{\partial f(\widetilde{T})}{\partial \widetilde{T}}$.

Assim, os termos $T^{\alpha}$ e $\exp \left(-\frac{T_{a}}{T}\right)$ após a filtragem são substituídos, respectivamente, por

$$
T^{\alpha}=\widetilde{T}^{\alpha}\left(1+\alpha \frac{T^{\prime \prime}}{\widetilde{T}}+\frac{\alpha(\alpha-1)}{2} \frac{T^{\prime \prime 2}}{\widetilde{T}^{2}}+O\left(T^{\prime \prime} 3\right)\right)=\widetilde{T}^{\alpha}\left(1+\sum_{n=1}^{\infty} Q_{n} \frac{T^{\prime \prime} n}{\widetilde{T}^{n}}\right), \mathrm{e}
$$




$$
\begin{aligned}
\exp \left(\frac{-T_{a}}{T}\right) & =\exp \left(\frac{-T_{a}}{\widetilde{T}}\right)\left[1+\frac{T_{a}}{\widetilde{T}} \frac{T^{\prime \prime}}{\widetilde{T}}+\left(\frac{T_{a}^{2}}{\widetilde{T}^{4}}-2 \frac{T_{a}}{\widetilde{T}^{3}}\right) \frac{T^{\prime \prime 2}}{2}+O\left(T^{\prime \prime} 3\right)\right] \\
& =\exp \left(\frac{-T_{a}}{\widetilde{T}}\right)\left(1+\sum_{n=1}^{\infty} P_{n} \frac{T^{\prime \prime} n}{\widetilde{T}^{n}}\right)
\end{aligned}
$$

onde $Q_{n}$ e $P_{n}$ são dados pelas expressões

$$
Q_{n}=\frac{1}{n !} \prod_{k=1}^{n}(\alpha-k-n) \text { e } P_{n}=\sum_{k=1}^{n} \frac{(n-1) !}{(n-k) ![(k-1) !]^{2} k}\left(\frac{T_{a}}{\widetilde{T}}\right)^{n} .
$$

Desta forma, o termo $\widetilde{\dot{\omega}_{C}}$ é dado por

$$
\begin{aligned}
\widetilde{\dot{\omega}_{C}} & =-A \overline{\rho^{2}} \widetilde{T^{\alpha}} \widetilde{Y_{C}} \widetilde{Y_{O}} \exp \left(\frac{-T_{a}}{\widetilde{T}}\right)\left[1+\frac{Y_{O}^{\prime \prime}}{\widetilde{Y_{O}}}+\frac{Y_{C}^{\prime \prime}}{\widetilde{Y_{C}}}+\frac{Y_{C}^{\prime \prime} Y_{O}^{\prime \prime}}{\widetilde{Y_{O}} \widetilde{Y_{C}}}+\left(P_{1}+Q_{1}\right) \times\right. \\
& \times\left(\frac{T^{\prime \prime}}{\widetilde{T}}+\frac{Y_{C}^{\prime \prime} T^{\prime \prime}}{\widetilde{T} \widetilde{Y_{C}}}+\frac{T^{\prime \prime} Y_{O}^{\prime \prime}}{\widetilde{Y_{O}} \widetilde{T}}+\frac{T^{\prime \prime} Y_{C}^{\prime \prime} Y_{O}^{\prime \prime}}{\widetilde{T} \widetilde{Y_{C}} \widetilde{Y_{O}}}\right)+\left(P_{2}+P_{1} Q_{1}+Q_{2}\right) \times \\
& \left.\times\left(\frac{T^{\prime \prime 2}}{\widetilde{T^{2}}}+\frac{Y_{C}^{\prime \prime} T^{\prime \prime 2}}{\widetilde{T^{2}} \widetilde{Y_{C}}}+\frac{T^{\prime \prime} 2 Y_{O}^{\prime \prime}}{\widetilde{Y_{O}} \widetilde{T^{2}}}+\frac{T^{\prime \prime} 2 Y_{C}^{\prime \prime} Y_{O}^{\prime \prime}}{\widetilde{T^{2}} \widetilde{Y_{C}} \widetilde{Y_{O}}}\right)+\ldots\right] .
\end{aligned}
$$

\section{A.2 Modelo da fração de mistura em escoamentos turbulentos}

Em um escoamento turbulento a equação de advecção-difusão da fração de mistura filtrada é dada pela equação (A.8). Entretanto os cálculos das frações mássicas das espécies químicas filtradas e da temperatura filtrada, por meio do modelo de fração de mistura, não são tão triviais quanto em um escoamento laminar. Para a simulação de escoamentos turbulentos, as relações lineares entre as frações mássicas das espécies químicas e a fração da mistura, descritas na Seção 2.3, relacionam os valores instantâneos e não os valores filtrados que são obtidos a partir das equações filtradas. $\mathrm{O}$ mesmo se aplica para a entalpia e para a temperatura. Para determinar as quantidades filtradas das frações mássicas das espécies e da temperatura é necessário utilizar a função densidade de probabilidade beta, $\beta$-pdf, como o fazem Versteeg e Malalasekera (2007), Apte et al. (2009), Pitsch (2006) e Pierce e Moin (2004), da seguinte maneira

$$
\widetilde{Y}_{l}=\int_{0}^{1} Y_{l}(y) P(y ; a, b) d y, \text { e } \widetilde{T}=\int_{0}^{1} T(y) P(y ; a, b) d y,
$$

onde $l=C, O, P$ e $P(y ; a, b)$ é a função $\beta$-pdf dada por

$$
P(y ; a, b)=\frac{y^{a-1}(1-y)^{b-1}}{\beta(a, b)}, \operatorname{com} \beta(a, b)=\frac{\Gamma(a) \Gamma(b)}{\Gamma(a+b)} \text { e } \beta(a, b)=\int_{0}^{1} y^{a-1}(1-y)^{b-1} d y,
$$

onde $\beta$ é a função beta e $\Gamma$ é a função Gamma. Os parâmetros $a$ e $b$ são determinados a partir do valor filtrado e da variância filtrada da fração da mistura, respectivamente, $\widetilde{z}$ e $\widetilde{z^{\prime}}$, como seguem 


$$
a=\widetilde{z}\left(\frac{\widetilde{z}(1-\widetilde{z})}{\widetilde{z^{\prime}}}-1\right), \text { e } b=(1-\widetilde{z}) \frac{a}{\widetilde{z}}
$$

A variância $\widetilde{z^{\prime}}$ é modelada segundo Pierce e Moin (2004) e Pitsch (2006), por meio da seguinte expressão

$$
\widetilde{z^{\prime}}=C_{z}(\Delta)^{2}|\nabla \widetilde{z}|^{2}
$$

onde $C_{z}$ é uma constante do modelo de turbulência, ou é modelado dinamicamente (Germano et al. (1991) e Pierce e Moin (2004)).

Do modelo de fração de mistura descrito na Seção 2.3 obtém-se as seguintes expressões para a espécie química combustível $Y_{C}$, oxidante $Y_{O}$ e produto $Y_{P}$ e para a temperatura $T$, dadas respectivamente por

$$
\begin{aligned}
& Y_{C}(z)= \begin{cases}0, & \text { se } 0 \leq z<z_{\text {est }}, \\
Y_{C}^{\star} \frac{z-z_{\text {est }}}{1-z_{\text {est }}}, & \text { se } z_{\text {est }} \leq z \leq 1,\end{cases} \\
& Y_{O}(z)= \begin{cases}Y_{O}^{\star}\left(1-\frac{z}{z_{\text {est }}}\right), & \text { se } 0 \leq z<z_{\text {est }}, \\
0, & \text { se } z_{\text {est }} \leq z \leq 1,\end{cases} \\
& Y_{P}(z)= \begin{cases}1-Y_{O}^{\star}\left(1-\frac{z}{z_{e s t}}\right), & \text { se } 0 \leq z<z_{e s t}, \\
1-Y_{C}^{\star} \frac{z-z_{e s t}}{1-z_{e s t}}, & \text { se } z_{e s t} \leq z \leq 1,\end{cases} \\
& T(z)= \begin{cases}z T_{C}^{\star}+(1-z) T_{O}^{\star}+\frac{\Delta h_{C}^{*} Y_{C}^{\star}}{c_{p}} z, & \text { se } 0 \leq z<z_{\text {est }}, \\
z T_{C}^{\star}+(1-z) T_{O}^{\star}+\frac{\Delta h_{C}^{*} Y_{C}^{\star}}{c_{p}} z_{\text {est }} \frac{1-z}{1-z_{\text {est }}}, & \text { se } z_{\text {est }} \leq z \leq 1 .\end{cases}
\end{aligned}
$$

onde $z_{\text {est }}$ é fração de mistura estequiométrica, $Y_{C}^{\star}$ é a fração mássica de combustível na entrada da zona de reação, $Y_{O}^{\star}$ é a fração mássica de oxidante na entrada da zona de reação, $T_{C}^{\star}$ é a temperatura do combustível na entrada da zona de reação, $T_{O}^{\star}$ é a temperatura do oxidante na entrada da zona de reação, $c_{p}$ é o calor específico à pressão constante e $\Delta h_{C}^{*}$ é a entalpia de formação da espécie química combustível. Substituindo-se as expressões (A.27)-(A.30) nas integrais descritas em (A.23) obtém-se, como em Cook e Riley (1994), as expressões

$$
\begin{aligned}
\widetilde{Y_{C}} & =\frac{Y_{C}^{\star}}{1-z_{e s t}}\left[\left(\frac{a}{a+b}\right) I_{1-z_{e s t}}(b, a+1)-I_{1-z_{e s t}}(b, a)\right], \\
\widetilde{Y_{O}} & =Y_{O}^{\star}\left[I_{z_{e s t}}(a, b)-\frac{1}{z_{e s t}}\left(\frac{a}{a+b}\right) I_{z_{e s t}}(a+1, b)\right], \\
\widetilde{Y_{P}} & =1-\widetilde{Y_{O}}-\widetilde{Y_{C}}
\end{aligned}
$$




$$
\begin{aligned}
\widetilde{T}= & \left(\frac{a}{a+b}\right) I_{z_{e s t}}(a+1, b)\left(T_{C}^{\star}+\frac{\Delta h_{C}^{*} Y_{C}^{\star}}{c_{p}}\right)+T_{O}^{\star}\left(\frac{b}{a+b}\right) I_{z_{e s t}}(a, b+1)+ \\
& +T_{C}^{\star}\left(\frac{a}{a+b}\right) I_{1-z_{e s t}}(b, a+1)+\left(\frac{b}{a+b}\right) I_{1-z_{e s t}}(b+1, a)\left(T_{O}^{\star}+\frac{\Delta h_{C}^{*} Y_{C}^{\star} z_{e s t}}{\left(1-z_{e s t}\right) c_{p}}\right),
\end{aligned}
$$

onde os parâmetros $a$ e $b$ são dados pela expressão (A.25). A função $I_{x}(a, b)$ é a função beta incompleta normalizada dada por

$$
I_{x}(a, b)=\frac{\beta_{x}(a, b)}{\beta(a, b)}=\frac{1}{\beta(a, b)} \int_{0}^{x} y^{a-1}(1-y)^{b-1} d y, \text { com } 0<x<1,
$$

onde $\beta_{x}(a, b)$ é a função beta incompleta.

A seguir mostra-se com detalhes como a expressão (A.31) para a fração mássica da espécie química combustível $Y_{C}$ é obtida. As demais expressões (A.32)-(A.34) são obtidas de maneira análoga.

Substituindo-se a expressão (A.25) na integral (A.23) obtém-se

$$
\widetilde{Y}_{C}=\frac{Y_{C}^{\star}}{\left(1-z_{\text {est }}\right) \beta(a, b)} \int_{z_{\text {est }}}^{1} z^{a}(1-z)^{b-1} d z-\frac{Y_{C}^{\star} z_{e s t}}{\left(1-z_{\text {est }}\right) \beta(a, b)} \int_{z_{\text {est }}}^{1} z^{a-1}(1-z)^{b-1} d z .
$$

Fazendo-se a mudança de variável $v=1-z$, na expressão (A.36) obtém-se

$$
\tilde{Y}_{C}=\frac{Y_{C}^{\star}}{\left(1-z_{e s t}\right) \beta(a, b)}\left[\int_{0}^{1-z_{e s t}}(1-v)^{a} v^{b-1} d v-z_{\text {est }} \int_{0}^{1-z_{\text {est }}}(1-v)^{a-1} v^{b-1} d v\right] .
$$

Multiplicando-se a primeira integral da expressão (A.37) por $\frac{\beta(b, a+1)}{\beta(b, a+1)}$ obtém-se

$$
\begin{aligned}
\tilde{Y}_{C} & =\frac{Y_{C}^{\star}}{\left(1-z_{\text {est }}\right)}\left[\frac{\beta(b, a+1)}{\beta(a, b)} \frac{1}{\beta(b, a+1)} \int_{0}^{1-z_{\text {est }}}(1-v)^{a} v^{b-1} d v+\right. \\
& \left.-\frac{z_{\text {est }}}{\beta(a, b)} \int_{0}^{1-z_{\text {est }}}(1-v)^{a-1} v^{b-1} d v\right] .
\end{aligned}
$$

Aplicando-se a definição da função beta incompleta normalizada e usando o fato que

$$
\frac{\beta(b, a+1)}{\beta(a, b)}=\frac{a \Gamma(a) \Gamma(b)}{(a+b) \Gamma(a+b)} \frac{\Gamma(a+b)}{\Gamma(a) \Gamma(b)}=\frac{a}{a+b},
$$

obtém-se

$$
\widetilde{Y}_{C}=\frac{Y_{C}^{\star}}{\left(1-z_{e s t}\right)}\left[\left(\frac{a}{a+b}\right) I_{1-z_{e s t}}(b, a+1)-z_{e s t} I_{1-z_{e s t}}(a, b)\right] .
$$




\section{Apêndice B}

\section{Sistema de equações rígido}

Para exemplificar o significado da rigidez do termo da reação química, considere o exemplo de um sistema de equações rígidas originário de um processo químico descrito por Robertson (1966) (veja Butcher (2003) e Hairer e Wanner (1991)). Um modelo de processo químico consistindo de três espécies químicas, as quais são denotadas por $A, B$ e $C$, é representado por

$$
\begin{aligned}
A & \longrightarrow B \\
B+C & \longrightarrow A+C \\
B+B & \longrightarrow C
\end{aligned}
$$

onde a seta " $\longrightarrow$ " representa o sentido da reação química. Sejam $Y_{A}, Y_{B}$ e $Y_{C}$ as frações mássicas das espécies $A, B$ e $C$ e $\dot{\omega}_{A}, \dot{\omega}_{B}$ e $\dot{\omega}_{C}$ as respectivas taxas de reação de cada espécie química. Por exemplo, a taxa de reação da primeira reação química na expressão (B.1) será denotada por $k_{A}$ indicando a taxa na qual $Y_{A}$ decresce e $Y_{B}$ aumenta. Na segunda reação, $k_{B}$ indica a produção da espécie química $A$ a partir da espécie $B$ e a fracão mássica $Y_{C}$ decresce a taxa de $k_{B} Y_{b} Y_{C}$. Na terceira reação, a produção da espécie $C$ a partir da espécie $B$ é dada por $k_{C} Y_{B}^{2}$. Desta forma, o processo de combustão (B.1) origina o seguinte sistema de equações diferenciais ordinárias

$$
\begin{aligned}
& \dot{\omega}_{A}=\frac{d Y_{A}}{d t}=-k_{A} Y_{A}+k_{B} Y_{C} \\
& \dot{\omega}_{B}=\frac{d Y_{B}}{d t}=k_{A} Y_{A}-k_{B} Y_{B} Y_{C}-k_{C} Y_{B}^{2} \\
& \dot{\omega}_{C}=\frac{d Y_{C}}{d t}=k_{C} Y_{B}^{2}
\end{aligned}
$$

sendo a condição inicial $Y_{A}(0)=1, Y_{B}(0)=0$ e $Y_{C}(0)=0$ e os parâmetros $k$ são as taxas da reação de cada reação química em (B.1).

O sistema (B.2) é denominado rígido quando uma ou mais variáveis "mudam rapidamente" em comparação às outras devido a existência de escalas de tempo diferentes, conforme Stoer e Bulirsch (2002) e Shampine e Gear (1979).

Se as taxas de reação $k_{A}, k_{B}$ e $k_{C}$ diferirem muito em magnitude o sistema (B.2) torna-se difícil de resolver numericamente. O exemplo de Robertson apresente taxas de reação $k_{A}=4 \times 10^{-2}$ (lenta), $k_{B}=10^{4}$ (rápida) e $k_{C}=3 \times 10^{7}$ (muito rápida), tornando-se difícil resolver esse sistema 
numericamente.

As frações mássicas das espécies químicas satisfazem $Y_{A}+Y_{B}+Y_{C}=1$. Desta forma, o sistema (B.2) pode ser reescrito como

$$
\begin{aligned}
\frac{d Y_{B}}{d t} & =k_{A}\left(1-Y_{B}-Y_{C}\right)-k_{B} Y_{B} Y_{C}-k_{C} Y_{B}^{2} \\
\frac{d Y_{C}}{d t} & =k_{C} Y_{B}^{2} .
\end{aligned}
$$

De maneira compacta,

$$
\frac{d \mathbf{Y}}{d t}=F(t, \mathbf{Y})
$$

Conforme Butcher (2003), sistemas de equações diferenciais rígidos são tipicamente não-lineares. Como a rigidez é associada ao comportamento das perturbações de uma dada solução, analisa-se a matriz Jacobiana. Considerando $\epsilon \mathbf{Z}$, uma perturbação da solução do sistema (B.3). Se $\mathbf{Y}$ é substituído por $\mathbf{Y}+\epsilon \mathbf{Z}$ em (B.4) e a solução é expandida em série de Taylor, obtém-se

$$
\frac{d \mathbf{Y}}{d t}+\epsilon \frac{d \mathbf{Z}}{d t}=F(t, \mathbf{Y})+\epsilon \frac{d F}{d \mathbf{Y}} \mathbf{Z}
$$

Subtraindo a expressão (B.4) de (B.5), obtém-se

$$
\dot{\mathbf{Z}}=\mathcal{J} \mathbf{Z} \text {. }
$$

A matriz Jacobiana do sistema (B.3) é dada por

$$
\mathcal{J}=\left[\begin{array}{cc}
-k_{A}-k_{B} Y_{c}-2 k_{c} Y_{B} & -k_{A}-k_{B} Y_{B} \\
2 k_{C} Y_{B} & 0
\end{array}\right] .
$$

O polinômio característico da matriz (B.7) é dado por

$$
p(\lambda)=\lambda^{2}+\left(k_{A}+k_{B} Y_{C}+2 k_{C} Y_{B}\right) \lambda+2 k_{C} Y_{B}\left(k_{A}+k_{B} Y_{B}\right) .
$$

Dessa forma os autovalores de $\mathcal{J}$ são

$$
\lambda=\frac{1}{2}\left[-\left(k_{A}+k_{B} Y_{C}+2 k_{C} Y_{B}\right) \pm \sqrt{\left(k_{A}+k_{B} Y_{C}+2 k_{C} Y_{B}\right)^{2}-8 k_{C} Y_{B}\left(k_{A}+k_{B} Y_{B}\right)}\right] .
$$

A parte real dos autovalores, $\operatorname{Re}(\lambda)$, de (B.3) é negativa já que $k_{A}, k_{B}, k_{C}, Y_{C}$ e $Y_{B}$ são sempre valores positivos. Para valores típicos de $Y_{B}$ e $Y_{C}$ tem-se $\operatorname{Re}(\lambda)<<0$, ou seja, negativo e de grande magnitude. Segundo Butcher (2003), a existência de um ou mais valores de $\lambda \Delta t$ de grande magnitude e negativos, com $\Delta t$ o intervalo de tempo entre duas soluções, é um sinal que o sistema é rígido. 


\section{Apêndice $\mathrm{C}$}

\section{Dedução das formas da equação da energia}

Este apêndice apresenta a dedução das diferentes formas da equação da conservação da energia descritas na Seção 2.1.4. A equação da conservação da energia total na notação indicial é dada por

$$
\frac{D e_{t}}{D t}=-\frac{\partial q_{j}}{\partial x_{j}}+\frac{\partial\left(\sigma_{i j} u_{i}\right)}{\partial x_{j}}=-\frac{\partial q_{j}}{\partial x_{j}}+\frac{\partial\left(\tau_{i j} u_{i}\right)}{\partial x_{j}}-\frac{\partial\left(p u_{i}\right)}{\partial x_{i}}
$$

onde $e_{t}$ é a energia total, $p$ é a pressão, $\mathbf{u}=\left(u_{1}, u_{2}, u_{3}\right)$ é o campo de velocidade, $\mathbf{q}=\left(q_{1}, q_{2}, q_{3}\right)$ é o fluxo de calor dado por

$$
q_{j}=-\kappa \frac{\partial T}{\partial x_{j}}+\rho \sum_{l=1}^{N} h_{l} Y_{l} v_{l, j}^{d},
$$

sendo $\kappa$ o coeficiente de difusão de calor, $T$ a temperatura, $h_{l}$ a entalpia absoluta da espécie química $l, Y_{l}$ a fração mássica da espécie química $l, \mathbf{v}_{l}^{d}=\left(v_{l, 1}^{d}, v_{l, 2}^{d}, v_{l, 3}^{d}\right)$ é a velocidade de difusão da espécie química $l$ e o tensor de tensões viscosas combinado com o tensor da pressão, $\sigma_{i j}$ dado por

$$
\sigma_{i j}=-p \delta_{i j}+\tau_{i j}=-p \delta_{i j}+\mu\left(\frac{\partial u_{i}}{\partial x_{j}}+\frac{\partial u_{j}}{\partial x_{i}}-\frac{2}{3} \delta_{i j} \frac{\partial u_{k}}{\partial x_{k}}\right)
$$

onde $\delta_{i j}$ é o delta de Kronecker.

A energia absoluta $e$ é obtida por meio da expressão,

$$
e=e_{t}-\frac{1}{2} \sum_{i=1}^{3} u_{i}^{2}
$$

Para obter-se a equação da conservação da energia absoluta é necessário obter a equação da conservação da energia cinética. Multiplicando-se a componente da velocidade $u_{i}$ na equação da conservação da quantidade de movimento (2.8), obtém-se

$$
\rho \frac{D}{D t}\left(\frac{1}{2} u_{1}^{2}\right)=-u_{1} \frac{\partial p}{\partial x_{1}}+u_{1} \frac{\partial \tau_{1 j}}{\partial x_{j}},
$$




$$
\begin{aligned}
\rho \frac{D}{D t}\left(\frac{1}{2} u_{2}^{2}\right) & =-u_{2} \frac{\partial p}{\partial x_{2}}+u_{2} \frac{\partial \tau_{2 j}}{\partial x_{j}}, \\
\rho \frac{D}{D t}\left(\frac{1}{2} u_{3}^{2}\right) & =-u_{3} \frac{\partial p}{\partial x_{3}}+u_{3} \frac{\partial \tau_{3 j}}{\partial x_{j}} .
\end{aligned}
$$

Subtraindo-se da equação (C.1) as equações (C.5)-(C.7) obtém-se na notação indicial a equação da conservação da energia absoluta, dada por

$$
\rho \frac{D e}{D t}=-p \frac{\partial u_{i}}{\partial x_{i}}+\frac{\partial}{\partial x_{j}}\left[\left(\kappa \frac{\partial T}{\partial x_{j}}\right)-\rho \sum_{l=1}^{N} h_{l} Y_{l} v_{l, j}^{d}\right]+\tau_{i j} \frac{\partial u_{i}}{\partial x_{j}} .
$$

A equação da conservação da entalpia absoluta é obtida por meio da expressão,

$$
h=e+\frac{p}{\rho} .
$$

Substituindo-se a expressão (C.9) no lado esquerdo da equação (C.8) obtém-se

$$
\begin{aligned}
\rho \frac{D e}{D t} & =\rho \frac{D h}{D t}-\rho \frac{D}{D t}\left(\frac{p}{\rho}\right), \\
& =\rho \frac{D h}{D t}-\frac{D p}{D t}+\frac{p}{\rho} \frac{D \rho}{D t}, \\
& =\rho \frac{D h}{D t}-\frac{D p}{D t}-p \frac{\partial u_{i}}{\partial x_{i}} .
\end{aligned}
$$

No terceiro passo da expressão (C.10) foi utilizada a equação da conservação da massa.

Assim, a equação da conservação da entalpia absoluta é dada por

$$
\rho \frac{D h}{D t}=\frac{D p}{D t}+\frac{\partial}{\partial x_{j}}\left[\left(\kappa \frac{\partial T}{\partial x_{j}}\right)-\rho \sum_{l=1}^{N} h_{l} Y_{l} v_{l, j}^{d}\right]+\tau_{i j} \frac{\partial u_{i}}{\partial x_{j}} .
$$

Seguindo a dedução feita por Turns (1996), o termo associado ao fluxo de calor,

$$
\dot{q}_{j}=-\kappa \frac{\partial T}{\partial x_{j}}+\rho \sum_{l=1}^{N} h_{l} Y_{l} v_{l, j}^{d}
$$

pode ser reescrito, utilizando-se a segunda Lei de Fick da Difusão descrita na Seção 2.1.3, da seguinte forma,

$$
\dot{q}_{j}=-\kappa \frac{\partial T}{\partial x_{j}}-\rho D \sum_{l=1}^{N} h_{l} \frac{\partial Y_{l}}{\partial x_{j}} .
$$

Aplicando-se a definição da derivada do produto no termo $\sum_{l} h_{l} Y_{l}$ tem-se

$$
\sum_{l=1}^{N} h_{l} \frac{\partial Y_{l}}{\partial x_{j}}=\frac{\partial}{\partial x_{j}}\left(\sum_{l=1}^{N} h_{l} Y_{l}\right)-\sum_{l=1}^{N} Y_{l} \frac{\partial h_{l}}{\partial x_{j}} .
$$


Desta forma, (C.13) torna-se

$$
\dot{q}_{j}=-\kappa \frac{\partial T}{\partial x_{j}}-\rho D \frac{\partial}{\partial x_{j}}\left(\sum_{l=1}^{N} h_{l} Y_{l}\right)+\rho D \sum_{l=1}^{N} Y_{l} \frac{\partial h_{l}}{\partial x_{j}}
$$

Como $c_{p l}=\frac{\partial h_{l}}{\partial T}$, o terceiro termo do lado direito de (C.11) em termos da temperatura $T$ e do calor específico à pressão constante $c_{p}$, torna-se

$$
\sum_{l=1}^{N} Y_{l} \frac{\partial h_{l}}{\partial x_{j}}=\sum_{l=1}^{N} Y_{l} c_{p l} \frac{\partial T}{\partial x_{j}}=c_{p} \frac{\partial T}{\partial x_{j}} .
$$

Usando a definição da entalpia absoluta da mistura $h=\sum_{l} Y_{l} h_{l},($ C.15) torna-se

$$
\dot{q}_{j}=-\kappa \frac{\partial T}{\partial x_{j}}-\rho D \frac{\partial h}{\partial x_{j}}+\rho D c_{p} \frac{\partial T}{\partial x_{j}} .
$$

Assumindo que o o número de Lewis é unitário, isto é, $\rho D c_{p}=\kappa$, a equação da conservação da entalpia absoluta é dada por

$$
\rho \frac{D h}{D t}=\frac{D p}{D t}+\frac{\partial}{\partial x_{j}}\left(\rho D \frac{\partial h}{\partial x_{j}}\right)+\tau_{i j} \frac{\partial u_{i}}{\partial x_{j}}
$$

Da definição de entalpia absoluta,

$$
h=\sum_{l=1}^{N} \Delta h_{l}^{*} Y_{l}+\int c_{p} d T
$$

onde $\Delta h_{l}^{*}$ é a entalpia de formação da espécie química $l$, obtém-se

$$
\begin{aligned}
\rho \frac{D h}{D t} & =\rho \frac{\partial h}{\partial T} \frac{D T}{D t}+\rho \sum_{l=1}^{N}\left(\Delta h_{l}^{*} \frac{D Y_{l}}{D t}\right) \\
& =\rho c_{p} \frac{D T}{D t}+\sum_{l=1}^{N} \Delta h_{l}^{*}\left[\dot{\omega}_{l}+\frac{\partial}{\partial x_{j}}\left(\rho D \frac{\partial Y_{l}}{\partial x_{j}}\right)\right], \\
& =\rho c_{p} \frac{D T}{D t}-\dot{\omega}_{T}-\frac{\partial}{\partial x_{i}}\left(\rho \sum_{l=1}^{N} \Delta h_{l}^{*} Y_{l} v_{l, j}^{d}\right),
\end{aligned}
$$

Em (C.20) foram utilizada a equação da conservação da fração mássica das espécies químicas na forma não conservativa, a definição da taxa de calor liberado devido a reação química, dada por

$$
\dot{\omega}_{T}=-\sum_{l=1}^{N} \Delta h_{l}^{*} \dot{\omega}_{l}
$$

e a segunda Lei de Fick da Difusão (2.13). 
Substituindo (C.20) em (C.11) obtém-se

$$
\rho c_{p} \frac{D T}{D t}=\frac{D p}{D t}+\frac{\partial}{\partial x_{j}}\left[\left(\kappa \frac{\partial T}{\partial x_{j}}\right)-\rho \sum_{l=1}^{N} h_{l} Y_{l} v_{l, j}^{d}\right]+\tau_{i j} \frac{\partial u_{i}}{\partial x_{j}}+\frac{\partial}{\partial x_{j}}\left(\rho \sum_{l=1}^{N} \Delta h_{l}^{*} Y_{l} v_{l, j}^{d}\right)+\dot{\omega}_{T} .
$$

Da definição de entalpia absoluta $h_{s l}=h_{l}-\Delta h_{l}^{*}$ a equação (C.22) pode ser reescrita como

$$
\rho c_{p} \frac{D T}{D t}=\frac{D p}{D t}+\frac{\partial}{\partial x_{j}}\left[\left(\kappa \frac{\partial T}{\partial x_{j}}\right)-\rho \sum_{l=1}^{N} h_{s l} Y_{l} v_{l, j}^{d}\right]+\tau_{i j} \frac{\partial u_{i}}{\partial x_{j}}+\dot{\omega}_{T}
$$

Conforme descrito no Capítulo 2 o termo $\tau_{i j} \frac{\partial u_{i}}{\partial x_{j}}$ não será considerado. Além disso, se $c_{p}=c_{p l}$ o termo $\sum_{l} h_{s l} Y_{l} v_{l, j}$ na equações (C.23) é nulo. Da definição de entalpia sensível tem-se que

$$
\sum_{l=1}^{N} h_{s l} Y_{l} v_{l, j}^{d}=\sum_{l=1}^{N}\left(\int_{T^{*}}^{T} c_{p l} d T\right) Y_{k} v_{l, j}^{d}=\left(\int_{T^{*}}^{T} c_{p} d T\right) \sum_{l=1}^{N} Y_{l} v_{l, j}^{d}=h_{s} \sum_{l=1}^{N} Y_{l} v_{l, j}^{d}=0 .
$$

O último termo é igual a zero já que $\sum_{l} Y_{l} v_{l, j}^{d}=0$ (veja Poinsot e Veynante (2005)).

Assim a equação de conservação da temperatura é dada por

$$
\rho c_{p} \frac{D T}{D t}=\frac{D p}{D t}+\frac{\partial}{\partial x_{j}}\left(\kappa \frac{\partial T}{\partial x_{j}}\right)+\dot{\omega}_{T}
$$




\section{Apêndice D}

\section{Adimensionalização das equações}

Este apêndice mostra como é feita a adimensionalização das equações da conservação da massa, das espécies químicas, da quantidade de movimento, da temperatura e da equação do estado, descritas na Seção 2.1,

$$
\begin{aligned}
\frac{\partial \rho}{\partial t}+\frac{\partial\left(\rho u_{j}\right)}{\partial x_{j}} & =0 \\
\frac{\partial\left(\rho Y_{l}\right)}{\partial t}+\frac{\partial\left(\rho u_{j} Y_{l}\right)}{\partial x_{j}} & =\frac{\partial}{\partial x_{j}}\left(\rho D \frac{\partial Y_{l}}{\partial x_{j}}\right)+\dot{\omega}_{l}, \\
\rho \frac{D u_{i}}{D t} & =\frac{\partial p}{\partial x_{i}}+\frac{\partial}{\partial x_{j}}\left[\mu\left(\frac{\partial u_{j}}{\partial x_{i}}+\frac{\partial u_{i}}{\partial x_{j}}-\frac{2}{3} \delta_{i j} \frac{\partial u_{k}}{\partial x_{k}}\right)\right], \\
\rho c_{p} \frac{D T}{D t} & =\frac{\partial}{\partial x_{j}}\left(\kappa \frac{\partial T}{\partial x_{j}}\right)+\frac{D p}{D t}+\dot{\omega}_{T}, \\
p & =\frac{\rho \mathcal{R} T}{W} .
\end{aligned}
$$

As propriedades adimensionais são dadas por,

$$
\rho^{a}=\frac{\rho}{\rho^{*}}, u_{i}^{a}=\frac{u_{i}}{u^{*}}, x_{i}^{a}=\frac{x_{i}}{L}, t^{a}=u^{*} \frac{t}{L}, \mu^{a}=\frac{\mu}{\mu^{*}}, T^{a}=\frac{T}{T^{*}}, p^{a}=\frac{p-p^{*}}{\rho^{*} u^{*} u^{*}}, D^{a}=\frac{D}{D^{*}},
$$

onde o símbolo "a" representa a propriedade adimensional e o símbolo "*", os valores de referência do escoamento. Substituindo as expressões da equação (D.6) nas equações (D.1)-(D.5) obtém-se as equações de conservação adimensionais.

\section{D.1 Equação da conservação da massa adimesional}

Substituindo-se $\rho=\rho^{a} \rho^{*}, u_{i}=u_{i}^{a} u^{*}, t=L \frac{t^{a}}{u^{*}}$ e $x_{i}=x_{i}^{a} L$ na equação (D.1) obtém-se,

$$
\frac{u^{*} \rho^{*}}{L} \frac{\partial \rho^{a}}{\partial t^{a}}+\frac{u^{*} \rho^{*}}{L} \frac{\partial\left(\rho^{a} u_{j}^{a}\right)}{\partial x_{j}^{a}}=0,
$$




$$
\frac{u^{*} \rho^{*}}{L}\left(\frac{\partial \rho^{a}}{\partial t^{a}}+\frac{\partial\left(\rho^{a} u_{j}^{a}\right)}{\partial x_{j}^{a}}\right)=0
$$

Portanto, a equação da conservação da massa específica adimensional é dada por,

$$
\frac{\partial \rho^{a}}{\partial t^{a}}+\frac{\partial\left(\rho^{a} u_{j}^{a}\right)}{\partial x_{j}^{a}}=0
$$

\section{D.2 Equação da conservação das espécies químicas adimensional}

Substituindo-se $\rho=\rho^{a} \rho^{*}, u_{i}=u_{i}^{a} u^{*}, t=L \frac{t^{a}}{u^{*}}, D=D^{a} D^{*}, \dot{\omega}_{l}=\dot{\omega}_{l}^{a} u^{*} \frac{\rho^{*}}{L}$ e $x_{i}=x_{i}^{a} L$ na equação (D.2) obtém-se,

$$
\begin{aligned}
\frac{u^{*} \rho^{*}}{L} \frac{\partial\left(\rho^{a} Y_{l}\right)}{\partial t^{a}}+\frac{u^{*} \rho^{*}}{L} \frac{\partial\left(\rho^{a} u_{j}^{a} Y_{l}\right)}{\partial x_{j}^{a}} & =\frac{\rho^{*} D^{*}}{L^{2}} \frac{\partial}{\partial x_{j}^{a}}\left(\rho^{a} D^{a} \frac{\partial Y_{l}}{\partial x_{j}^{a}}\right)+\frac{u^{*} \rho^{*}}{L} \dot{\omega}_{l}^{a}, \\
\frac{u^{*} \rho^{*}}{L}\left(\frac{\partial\left(\rho^{a} Y_{l}\right)}{\partial t^{a}}+\frac{\partial\left(\rho^{a} u_{j}^{a} Y_{l}\right)}{\partial x_{j}^{a}}\right) & =\frac{\rho^{*} D^{*} \mu^{*}}{\mu^{*} L^{2}} \frac{\partial}{\partial x_{j}^{a}}\left(\rho^{a} D^{a} \frac{\partial Y_{l}}{\partial x_{j}^{a}}\right)+\frac{u^{*} \rho^{*}}{L} \dot{\omega}_{l}^{a}, \\
\frac{\partial\left(\rho^{a} Y_{l}\right)}{\partial t^{a}}+\frac{\partial\left(\rho^{a} u_{j}^{a} Y_{l}\right)}{\partial x_{j}^{a}} & =\frac{L}{\rho^{*} u^{*}}\left[\frac{\rho^{*} D^{*} \mu^{*}}{\mu^{*} L^{2}} \frac{\partial}{\partial x_{j}^{a}}\left(\rho^{a} D^{a} \frac{\partial Y_{l}}{\partial x_{j}^{a}}\right)+\frac{u^{*} \rho^{*}}{L} \dot{\omega}_{l}^{a}\right], \\
& =\frac{\mu^{*}}{\rho^{*} u^{*} L} \frac{\rho^{*} D^{*}}{\mu^{*}} \frac{\partial}{\partial x_{j}^{a}}\left(\rho^{a} D^{a} \frac{\partial Y_{l}}{\partial x_{j}^{a}}\right)+\dot{\omega}_{l}^{a} .
\end{aligned}
$$

Portanto, a equação da conservação da fração mássica da espécie $l$ adimensional é dada por,

$$
\frac{\partial\left(\rho^{a} Y_{l}\right)}{\partial t^{a}}+\frac{\partial\left(\rho^{a} u_{j}^{a} Y_{l}\right)}{\partial x_{j}^{a}}=\frac{1}{R e} \frac{1}{S c} \frac{\partial}{\partial x_{j}^{a}}\left(\rho^{a} D^{a} \frac{\partial Y_{l}}{\partial x_{j}^{a}}\right)+\dot{\omega}_{l}^{a} .
$$

onde $R e$ é o número de Reynolds e $S c$ é o número de Schmidt, definidos por, $R e=\frac{\rho L u}{\mu}$ e $S c=\frac{\mu}{\rho D}$.

\section{D.3 Equação da quantidade de movimento linear adimensional}

A equação da quantidade de movimento linear pode ser adimensionalizada de duas maneiras. Na primeira, substitui-se $\rho=\rho^{a} \rho^{*}, u_{i}=u_{i}^{a} u^{*}, t=L \frac{t^{a}}{u^{*}}, \mu=\mu^{a} \mu^{*}, x_{i}=x_{i}^{a} L$ e $p=p^{a} \rho^{*} u^{*} u^{*}+p^{*}$ na equação (D.3) e obtém-se

$$
\begin{aligned}
\frac{\rho^{*} u^{*} u^{*}}{L} \frac{\partial \rho^{a} u_{i}^{a}}{\partial t^{a}}+\frac{\rho^{*} u^{*} u^{*}}{L} \frac{\partial \rho u_{i} u_{j}}{\partial x_{j}^{a}} & =\frac{\rho^{*} u^{*} u^{*}}{L} \frac{\partial p^{a}}{\partial x_{i}^{a}}+\frac{\mu^{*} u^{*}}{L^{2}} \frac{\partial}{\partial x_{j}^{a}}\left[\mu^{a}\left(\frac{\partial u^{a}}{\partial x_{j}^{a}}+\frac{\partial u_{j}^{a}}{\partial x_{i}^{a}}-\frac{2}{3} \delta_{i j} \frac{\partial u_{k}^{a}}{\partial x_{k}^{a}}\right)\right], \\
\rho^{a}\left(\frac{\partial u_{i}^{a}}{\partial t^{a}}+u_{j}^{a} \frac{\partial u_{i}^{a}}{\partial x_{j}^{a}}\right) & =-\frac{p^{*}}{\rho^{*} u^{*}} \frac{\partial p^{a}}{\partial x_{i}^{a}}+\frac{\mu^{*}}{\rho^{*} L u^{*}} \frac{\partial}{\partial x_{j}^{a}}\left[\mu^{a}\left(\frac{\partial u_{i}^{a}}{\partial x_{j}^{a}}+\frac{\partial u_{j}^{a}}{\partial x_{i}^{a}}-\frac{2}{3} \delta_{i j} \frac{\partial u_{k}^{a}}{\partial x_{k}^{a}}\right)\right] .
\end{aligned}
$$


Portanto, a equação da quantidade de movimento linear adimensional é dada por,

$$
\rho^{a}\left(\frac{\partial u_{i}^{a}}{\partial t^{a}}+u_{j}^{a} \frac{\partial u_{i}^{a}}{\partial x_{j}^{a}}\right)=-\frac{\partial p^{a}}{\partial x_{i}^{a}}+\frac{1}{R e} \frac{\partial}{\partial x_{j}^{a}}\left[\mu^{a}\left(\frac{\partial u^{a}}{\partial x_{j}^{a}}+\frac{\partial u_{j}^{a}}{\partial x_{i}^{a}}-\frac{2}{3} \delta_{i j} \frac{\partial u_{k}^{a}}{\partial x_{k}^{a}}\right)\right] .
$$

Na segunda, substitui-se todas as propriedades como na maneira anterior, com exceção da pressão que será adimensionalizada como $p=p^{*} p^{a}$. Desta forma, obtém-se

$$
\begin{aligned}
\frac{\rho^{*} u^{*} u^{*}}{L} \frac{\partial \rho^{a} u_{i}^{a}}{\partial t^{a}}+\frac{\rho^{*} u^{*} u^{*}}{L} \frac{\partial \rho u_{i} u_{j}}{\partial x_{j}^{a}} & =\frac{p^{*}}{L} \frac{\partial p^{a}}{\partial x_{i}^{a}}+\frac{\mu^{*} u^{*}}{L^{2}} \frac{\partial}{\partial x_{j}^{a}}\left[\mu^{a}\left(\frac{\partial u^{a}}{\partial x_{j}^{a}}+\frac{\partial u_{j}^{a}}{\partial x_{i}^{a}}-\frac{2}{3} \delta_{i j} \frac{\partial u_{k}^{a}}{\partial x_{k}^{a}}\right)\right], \\
\rho^{a}\left(\frac{\partial u_{i}^{a}}{\partial t^{a}}+u_{j}^{a} \frac{\partial u_{i}^{a}}{\partial x_{j}^{a}}\right) & =-\frac{p^{*}}{\rho^{*} u^{*} u^{*}} \frac{\partial p^{a}}{\partial x_{i}^{a}}+\frac{\mu^{*}}{\rho^{*} L u^{*}} \frac{\partial}{\partial x_{j}^{a}}\left[\mu^{a}\left(\frac{\partial u_{i}^{a}}{\partial x_{j}^{a}}+\frac{\partial u_{j}^{a}}{\partial x_{i}^{a}}-\frac{2}{3} \delta_{i j} \frac{\partial u_{k}^{a}}{\partial x_{k}^{a}}\right)\right] .
\end{aligned}
$$

Portanto, a equação da quantidade de movimento linear adimensional é dada por,

$$
\rho^{a}\left(\frac{\partial u_{i}^{a}}{\partial t^{a}}+u_{j}^{a} \frac{\partial u_{i}^{a}}{\partial x_{j}^{a}}\right)=-\frac{1}{\gamma M a^{2}} \frac{\partial p^{a}}{\partial x_{i}^{a}}+\frac{1}{R e} \frac{\partial}{\partial x_{j}^{a}}\left[\mu^{a}\left(\frac{\partial u^{a}}{\partial x_{j}^{a}}+\frac{\partial u_{j}^{a}}{\partial x_{i}^{a}}-\frac{2}{3} \delta_{i j} \frac{\partial u_{k}^{a}}{\partial x_{k}^{a}}\right)\right],
$$

onde $M a$ é o número de Mach definido por $M a=\frac{u^{*}}{\sqrt{\gamma \frac{p^{*}}{\rho^{*}}}}$ e $\gamma$ é a razão entre a capacidade de calor à pressão constante e a capacidade de calor a volume constante, $\gamma=\frac{c_{p}}{c_{v}}$.

\section{D.4 Equação da temperatura adimensional}

A equação da temperatura adimensional também pode ser obtida de duas maneiras. Na primeira, substituindo-se $\rho=\rho^{a} \rho^{*}, u_{i}=u_{i}^{a} u^{*}, t=L \frac{t^{a}}{u^{*}}, \kappa=\kappa^{a} \kappa^{*}, x_{i}=x_{i}^{a} L$ e $p=p^{a} \rho^{*} u^{*} u^{*}+p^{*}$ na equação (D.4), obtém-se

$$
\begin{aligned}
\frac{c_{p}^{*} \rho^{*} T^{*} u^{*}}{L} \rho^{a} c_{p}^{a} \frac{D T^{a}}{D t^{a}} & =\frac{\rho^{*} u^{*} u^{*} u^{*}}{L} \frac{D p^{a}}{D t^{a}}+\frac{\kappa^{*} T^{*}}{L^{2}} \frac{\partial}{\partial x_{j}^{a}}\left(\kappa^{a} \frac{\partial T^{a}}{\partial x_{j}^{a}}\right)+\frac{u^{*} \rho^{*} T^{*} c_{p}^{*}}{L} \dot{\omega}_{T}, \\
\rho^{a} c_{p}^{a} \frac{D T^{a}}{D t^{a}} & =-\frac{u^{*} u^{*}}{c_{p}^{*} T^{*}} \frac{D p^{a}}{D t^{a}}+\frac{\kappa^{*}}{c_{p}^{*} \mu^{*}} \frac{\mu^{*}}{\rho^{*} u^{*} L} \frac{\partial}{\partial x_{j}^{a}}\left(\kappa^{a} \frac{\partial T^{a}}{\partial x_{j}^{a}}\right)+\dot{\omega}_{T}, \\
& =\frac{\gamma \mathcal{R}^{*} u^{*} u^{*}}{c_{p}^{*} \gamma \mathcal{R}^{*} T^{*}} \frac{D p^{a}}{D t^{a}}+\frac{\kappa^{*}}{c_{p}^{*} \mu^{*}} \frac{\mu^{*}}{\rho^{*} u^{*} L} \frac{\partial}{\partial x_{j}^{a}}\left(\kappa^{a} \frac{\partial T^{a}}{\partial x_{j}^{a}}\right)+\dot{\omega}_{T}, \\
& =\frac{\gamma \mathcal{R}^{*} M a^{2}}{c_{p}^{*}} \frac{D p^{a}}{D t^{a}}+\frac{1}{\operatorname{Pr}} \frac{1}{\operatorname{Re}} \frac{\partial}{\partial x_{j}^{a}}\left(\kappa^{a} \frac{\partial T^{a}}{\partial x_{j}^{a}}\right)+\dot{\omega}_{T}, \\
& =(\gamma-1) M a^{2} \frac{D p^{a}}{D t^{a}}+\frac{1}{\operatorname{Pr}} \frac{1}{R e} \frac{\partial}{\partial x_{j}^{a}}\left(\kappa^{a} \frac{\partial T^{a}}{\partial x_{j}^{a}}\right)+\dot{\omega}_{T} .
\end{aligned}
$$


Portanto a equação da temperatura adimensional é dada por

$$
\rho^{a} c_{p}^{a} \frac{D T^{a}}{D t^{a}}=(\gamma-1) M a^{2} \frac{D p^{a}}{D t^{a}}+\frac{1}{\operatorname{Pr}} \frac{1}{R e} \frac{\partial}{\partial x_{j}^{a}}\left(\kappa^{a} \frac{\partial T^{a}}{\partial x_{j}^{a}}\right)+\dot{\omega}_{T},
$$

sendo $\operatorname{Pr}=\frac{\mu c_{p}}{\kappa}$ o número de Prandtl e $M a=\frac{u^{*}}{\sqrt{\gamma \mathcal{R}^{*} T^{*}}}$ o número de Mach. Para obter a equação da temperatura adimensional (D.11) foram utilizadas as relações $R_{M}=\frac{\mathcal{R}}{W^{*} W^{a}}=\frac{\mathcal{R}^{*}}{W^{a}} \mathrm{e} \frac{c_{p}}{\mathcal{R}^{*}}=\frac{\gamma}{\gamma-1}$.

Na segunda maneira de adimensionalizar, substitui-se na equação (D.4), todas as variáveis como anteriormente, com exceção da pressão que será substituida por $p=p^{a} p^{*}$. Desta forma, obtém-se

$$
\begin{aligned}
\frac{c_{p}^{*} \rho^{*} T^{*} u^{*}}{L} \rho^{a} c_{p}^{a} \frac{D T^{a}}{D t^{a}} & =\frac{u^{*} p^{*}}{L} \frac{D p^{a}}{D t^{a}}+\frac{\kappa^{*} T^{*}}{L^{2}} \frac{\partial}{\partial x_{j}^{a}}\left(\kappa^{a} \frac{\partial T^{a}}{\partial x_{j}^{a}}\right)+\frac{u^{*} \rho^{*} T^{*} c_{p}^{*}}{L} \dot{\omega}_{T}, \\
\rho^{a} c_{p}^{a} \frac{D T^{a}}{D t^{a}} & =-\frac{p *}{c_{p}^{*} \rho^{*} T^{*}} \frac{D p^{a}}{D t^{a}}+\frac{\kappa^{*}}{c_{p}^{*} \mu^{*}} \frac{\mu^{*}}{\rho^{*} u^{*} L} \frac{\partial}{\partial x_{j}^{a}}\left(\kappa^{a} \frac{\partial T^{a}}{\partial x_{j}^{a}}\right)+\dot{\omega}_{T}, \\
& =\frac{\mathcal{R}^{*} p^{*}}{c_{p}^{*} \rho^{*} \mathcal{R}^{*} T^{*}} \frac{D p^{a}}{D t^{a}}+\frac{\kappa^{*}}{c_{p}^{*} \mu^{*}} \frac{\mu^{*}}{\rho^{*} u^{*} L} \frac{\partial}{\partial x_{j}^{a}}\left(\kappa^{a} \frac{\partial T^{a}}{\partial x_{j}^{a}}\right)+\dot{\omega}_{T}, \\
& =\frac{\mathcal{R}^{*}}{c_{p}^{*}} \frac{D p^{a}}{D t^{a}}+\frac{1}{\operatorname{Pr}} \frac{1}{R e} \frac{\partial}{\partial x_{j}^{a}}\left(\kappa^{a} \frac{\partial T^{a}}{\partial x_{j}^{a}}\right)+\dot{\omega}_{T}, \\
& =\frac{\gamma-1}{\gamma} \frac{D p^{a}}{D t^{a}}+\frac{1}{\operatorname{Pr}} \frac{1}{R e} \frac{\partial}{\partial x_{j}^{a}}\left(\kappa^{a} \frac{\partial T^{a}}{\partial x_{j}^{a}}\right)+\dot{\omega}_{T} .
\end{aligned}
$$

Portanto, a equação da temperatura adimensional é dada por,

$$
\rho^{a} c_{p}^{a} \frac{D T^{a}}{D t^{a}}=\frac{\gamma-1}{\gamma} \frac{D p^{a}}{D t^{a}}+\frac{1}{\operatorname{Pr}} \frac{1}{R e} \frac{\partial}{\partial x_{j}^{a}}\left(\kappa^{a} \frac{\partial T^{a}}{\partial x_{j}^{a}}\right)+\dot{\omega}_{T} .
$$

Para obter a equação (D.12) utilizamos o fato que $p^{*}=\rho^{*} \mathcal{R}^{*} T^{*}$.

\section{D.5 Equação do estado termodinâmico adimensional}

Como na equação da quantidade de movimento linear adimensional e a da temperatura adimensional, a equação do estado termodinâmico também pode ser adimensionalizada de duas maneiras. Na primeira, substituindo -se $\rho=\rho^{a} \rho^{*}, T=T^{a} T^{*}, p=p^{a} \rho^{*} u^{*} u^{*}+p^{*}, W=W^{*} W^{a}$ na equação (D.5), tem-se

$$
\begin{aligned}
p^{a} \rho^{*} u^{*} u^{*}+p^{*} & =\frac{\rho^{*} \rho^{a} \mathcal{R} T^{*} T^{a}}{W^{*} W^{a}}, \\
\frac{\rho^{a} T^{a}}{W^{a}} & =\frac{p^{a} \rho^{*} u^{*} u^{*}+p^{*}}{\rho^{*} \mathcal{R}^{*} T^{*}}, \\
& =\frac{p^{a} \gamma u^{*} u^{*}+p^{*}}{\gamma \mathcal{R}^{*} T^{*}}, \\
& =\gamma M a^{2} p^{a}+1 .
\end{aligned}
$$


Portanto,

$$
\frac{\rho^{a} T^{a}}{W^{a}}=\gamma M a^{2} p^{a}+1
$$

Na segunda maneira, substituindo-se $\rho=\rho^{a} \rho^{*}, T=T^{a} T^{*}, p=p^{a} p^{*}, W=W^{*} W^{a}$, na equação (D.5) tem-se

$$
\begin{aligned}
p^{a} p^{*} & =\frac{\rho^{*} \rho^{a} \mathcal{R} T^{*} T^{a}}{W^{*} W^{a}}, \\
\frac{\rho^{a} T^{a}}{W^{a}} & =\frac{p^{a} p^{*}}{\rho^{*} \mathcal{R}^{*} T^{*}} .
\end{aligned}
$$

Portanto,

$$
\frac{\rho^{a} T^{a}}{W^{a}}=p^{a}
$$




\section{Referências Bibliográficas}

Apte et al.(2009) S. V. Apte, K. Mahesh e P. Moin. Large-eddy simulation of evaporating spray in a coaxial combustor. Proceedings of the Combustion Institute, 32(2):2247-2256. Citado na pág. $2,3,18,92,102$

Ascher et al.(1995) U. M. Ascher, S. J. Ruuth e B. T. R. Wetton. Implicit-explicit methods for time-dependent partial differential equations. SIAM Journal on Numerical Analysis, páginas 797-823. Citado na pág. 1, 2, 25, 28

Balow e Frank(2007) R. Balow e J. Frank. Piloted ch4/air flames c, d, e, and f- release 2.1. www.ca.sandia.gov/TNF. Citado na pág. $65,67,83,84,86,87$

Batchelor(2000) G.K. Batchelor. An introduction to fluid dynamics. Cambridge University Press. Citado na pág. 8, 9

Bell(2005) J. Bell. Amr for low mach number reacting flow. Adaptive Mesh Refinement-Theory and Applications, páginas 203-221. Citado na pág. 4, 5, 7, 10

Bell et al.(1989) J.B. Bell, P. Colella e H.M. Glaz. A second-order projection method for the incompressible navier-stokes equations. Journal of Computational Physics, 85(2):257-283. Citado na pág. 26,36

Berger e Rigoutsos(1991) M. Berger e I. Rigoutsos. An algorithm for point clustering and grid generation. Systems, Man and Cybernetics, IEEE Transactions on, 21(5):1278-1286. Citado na pág. $4,45,48,49$

Berger e Oliger(1984) M. J. Berger e J. Oliger. Adaptive mesh refinement for hyperbolic partial differential equations. Journal of computational Physics, 53(3):484-512. Citado na pág. 1, 2, 4, 45

Berger e Colella(1989) M.J. Berger e P. Colella. Local adaptive mesh refinement for shock hydrodynamics. Journal of computational Physics, 82(1):64-84. Citado na pág. 1, 4, 45, 48

Bhatia e Durst(1989) J. C. Bhatia e F. Durst. Comparative study of some probability distributions applied to liquid sprays. Particle 8 Particle Systems Characterization, 6(1-4):151-162. Citado na pág. 79,83

Bini e Jones(2009) M. Bini e W. P. Jones. Large eddy simulation of an evaporating acetone spray. International Journal of Heat and Fluid Flow, 30(3):471-480. Citado na pág. 5, 20, 92

Boersma et al.(1998) BJ Boersma, G. Brethouwer e FTM Nieuwstadt. A numerical investigation on the effect of the inflow conditions on the self-similar region of a round jet. Physics of fluids, 10:899. Citado na pág. $65,67,70,71,86,91$

Briggs e McCormick(2000) W. L. Briggs e S. F. McCormick. A multigrid tutorial, volume 72. Society for Industrial Mathematics. Citado na pág. 25, 35

Burden e Faires(2005) R. L. Burden e J. D. Faires. Numerical Analysis. Thomson Brooks cole. Citado na pág. 40 
Butcher(2003) J. C. Butcher. Numerical Methods for Ordinary Differential Equations. Wiley. Citado na pág. 10, 105, 106

Ceniceros et al.(2010a) H.D. Ceniceros, R.L. Nós e A.M. Roma. Three-dimensional, fully adaptive simulations of phase-field fluid models. Journal of Computational Physics, 229(17):61356155. Citado na pág. 1, 4

Ceniceros et al.(2010b) H.D. Ceniceros, A.M. Roma, A. Silveira-Neto e M.M. Villar. A robust, fully adaptive hybrid level-set/front-tracking method for two-phase flows with an accurate surface tension computation. Communications in Compututational Physics, 8(1):51-94. Citado na pág. 1, $4,21,25$

Chorin(1968) A. J. Chorin. Numerical solution of the navier-stokes equations. Math. Comp, 22 (104):745-762. Citado na pág. 25, 26, 58

Chorin e Marsden(1993) A.J. Chorin e J.E. Marsden. A mathematical introduction to fluid mechanics, volume 4. Springer. Citado na pág. 8, 9, 10, 26

Chrigui et al.(2012) M. Chrigui, J. Gounder, A. Sadiki, A.R. Masri e J. Janicka. Partially premixed reacting acetone spray using les and fgm tabulated chemistry. Combustion and Flame, páginas 2718-2741. Citado na pág. 5

Cook e Riley(1994) A.W. Cook e J.J. Riley. A subgrid model for equilibrium chemistry in turbulent flows. Physics of fluids, 6:2868. Citado na pág. 103

Crowe et al.(1998) C. T. Crowe, M. Sommerfeld e Y. Tsuji. Multiphase flows with droplets and particles. CRC. Citado na pág. 3, 7, 18, 19, 77, 79, 83

Day e Bell(2000) M.S. Day e J.B. Bell. Numerical simulation of laminar reacting flows with complex chemistry. Combustion Theory and Modelling, 4(4):535-556. Citado na pág. 2, 3, 4, 5, 7, $83,84,85,86$

De et al.(2011) S. De, K. N. Lakshmisha e R. W. Bilger. Modeling of nonreacting and reacting turbulent spray jets using a fully stochastic separated flow approach. Combustion and Flame. Citado na pág. $4,5,6,7,65,77,78,83,86,91$

Douglas e Dupont(1971) J. Douglas e T. Dupont. Alternating-direction galerkin methods on rectangles. Numerical Solution of Partial Differential Equations, II (SYNSPADE 1970), páginas 133-214. Citado na pág. 29

Feofiloff(2009) P. Feofiloff. Algoritmos em linguagem C. Elsevier Brazil. Citado na pág. 50

Ferziger e Perić(1999) J. H. Ferziger e M. Perić. Computational methods for fluid dynamics, volume 2. Springer Berlin. Citado na pág. 26, 33

Fortuna(2000) A. O. Fortuna. Técnicas computacionais para dinâmica dos fluidos: conceitos básicos e aplicações. Edusp. Citado na pág. 31

Gao e Groth(2008) X. Gao e CPT Groth. Parallel adaptive mesh refinement scheme for threedimensional turbulent non-premixed combustion. Em 46th AIAA Aerospace Sciences Meeting and Exhibit, Reno, Nevada, AIAA paper, volume 1017, página 2008. Citado na pág. 2, 3, 5

García(2009) M. M. García. Development and validation of the Euler-Lagrange formulation on a parallel and unstructured solver for large-eddy simulation. Tese de Doutorado, Institut National Polytechnique de Toulouse. Citado na pág. 2, 3, 5, 7, 18, 77, 79

Germano et al.(1991) M. Germano, U. Piomelli, P. Moin e W.H. Cabot. A dynamic subgrid-scale eddy viscosity model. Physics of Fluids A: Fluid Dynamics, 3:1760. Citado na pág. 75, 100, 103 
Gottlieb e Orszag(1993) D. Gottlieb e S.A. Orszag. Numerical analysis of spectral methods: theory and applications, volume 26. Society for industrial and applied mathematics. Citado na pág. 29

Gounder e Masri(2009) J.D. Gounder e A.R. Masri. Flow field and mass flux measurements near the exit plane of spray jets. Em 11th Triennial International Annual Conference on Liquid Atomization and Spray Systems, ICLASS. Citado na pág. 77, 78

Griffith(2005) B. E. Griffith. Simulating the blood-muscle-valve mechanics of the heart by an adaptive and parallel version of the immersed boundary method. Tese de Doutorado, New York University. Citado na pág. 25, 26, 41, 58

Hackbusch(1992) W. Hackbusch. Elliptic differential equations, volume 18. Springer. Citado na pág. 38

Hairer e Wanner(1991) H. Hairer e G. Wanner. Solving ordinary differential equations II: stiff and differential - algebraic problems. Springer. Citado na pág. 105

Hou e Mahesh(2005) Y. Hou e K. Mahesh. A robust, colocated, implicit algorithm for direct numerical simulation of compressible, turbulent flows. Journal of Computational Physics, 205 (1):205-221. Citado na pág. 12

Hussein et al.(1994) H.J. Hussein, S.P. Capp e W.K. George. Velocity measurements in a highreynolds-number, momentum-conserving, axisymmetric, turbulent jet. Journal of Fluid Mechanics, 258(1):31-75. Citado na pág. $65,71,74$

Kempf et al.(2005) A. Kempf, F. Flemming e J. Janicka. Investigation of lenghtscales, scalar dissipation, and flame orientation in a piloted diffusion flame by les. Bull. Soc. Math. France, 98:115-152. Citado na pág. $65,67,83,86$

Kim e Moin(1985) J. Kim e P. Moin. Application of a fractional-step method to incompressible navier-stokes equations. Journal of Computational Physics, 59(2):308-323. Citado na pág. 26

Kuo(2005) K. K. Kuo. Principles of combustion. Wiley. Citado na pág. 3, 7, 8, 9, 10, 11, 12, 17, 18, 67,84

Lefebvre(1989) A.H. Lefebvre. Atomization and sprays. CRC. Citado na pág. 79, 83

Majda e Sethian(1985) A. Majda e J. Sethian. The derivation and numerical solution of the equations for zero mach number combustion. Combustion science and technology, 42(3):185-205. Citado na pág. 4, 7, 14, 27

Melo e Neto(1991) S. T. Melo e F. M. Neto. Mecânica dos fluidos e equações diferenciais. IMPA. Citado na pág. 8,9

Mood et al.(1974) A. M. Mood, F.A. Graybill e D.C. Boes. Introduction to the Theory of Statistics. New York: McGraw-Hill, 3rd edição. Citado na pág. 79

Najm e Knio(2005) HN Najm e OM Knio. Modeling low mach number reacting flow with detailed chemistry and transport. Journal of Scientific Computing, 25(1):263-287. Citado na pág. 3

Nicoud(2000) F. Nicoud. Conservative high-order finite-difference schemes for low-mach number flows. Journal of Computational Physics, 158(1):71-97. Citado na pág. 4, 7

Nonaka et al.(2012) A. Nonaka, AJ Aspden, M. Zingale, AS Almgren, JB Bell e SE Woosley. High-resolution simulations of convection preceding ignition in type ia supernovae using adaptive mesh refinement. The Astrophysical Journal, 745:73. Citado na pág. 5 
Northrup(2004) S.A. Northrup. A Parallel Adaptive-Mesh Refinement Scheme for Predicting Laminar Diffusion Flames. Tese de Doutorado, University of Toronto. Citado na pág. 84, 85, 86

Northrup e Groth(2009) S.A. Northrup e C.P.T. Groth. Solution of laminar combusting flows using a parallel implicit adaptive mesh refinement algorithm. Computational Fluid Dynamics 2006, páginas 341-346. Citado na pág. 5, 83

Nós(2007) R. L. Nós. Simulações de escoamentos tridimensionais bifásicos empregando métodos adaptativos e modelos de campo de fase. Tese de Doutorado, Universidade de São Paulo. Citado na pág. xi, 1, 4, 21, 25, 29, 34, 35, 36, 37, 45, 46, 52, 56, 57, 58

Pember et al.(1998) R. B. Pember, L. H. Howell, J. B. Bell, P. Colella, W. Y. Crutchfield, W. A. Fiveland e J. P. Jessee. An adaptive projection method for unsteady, low-mach number combustion. Combustion Science and Technology, 140(1):123-168. Citado na pág. 2, 3, 4, 5, 7, 10, $25,27,36,60,66,84$

Pierce e Moin(2004) C.D. Pierce e P. Moin. Progress-variable approach for large-eddy simulation of non-premixed turbulent combustion. Journal of Fluid Mechanics, 504(1):73-97. Citado na pág. $3,92,100,102,103$

Pitsch(2006) H. Pitsch. Large-eddy simulation of turbulent combustion. Annu. Rev. Fluid Mech., 38:453-482. Citado na pág. 100, 102, 103

Pitsch e Steiner(2000) H. Pitsch e H. Steiner. Large-eddy simulation of a turbulent piloted methane/air diffusion flame (sandia flame d). Physics of Fluids, 12:2541. Citado na pág. 2, 65, 67, $83,84,86,87,92$

Plewa et al.(2005) T. Plewa, T.J. Linde e V.G. Weirs. Adaptive mesh refinement, theory and applications: proceedings of the Chicago Workshop on Adaptive Mesh Refinement Methods, Sept. 3-5, 2003, volume 41. Springer Verlag. Citado na pág. 4

Poinsot e Veynante(2005) T. Poinsot e D. Veynante. Theoretical and numerical combustion. RT Edwards, Inc. Citado na pág. 3, 7, 8, 10, 11, 12, 13, 16, 17, 18, 60, 99, 100, 101, 110

Pope(2000) S.B. Pope. Turbulent flows. Cambridge University Press. Citado na pág. 66, 75, 99, 100

Rehm e Baum(1978) R.G. Rehm e H.R. Baum. The equations of motion for thermally driven, buoyant flows. Journal of research of the National Bureau of Standards, 83(3):297-308. Citado na pág. $4,7,14,27$

Roma(1996) A. M. Roma. A multilevel self adaptative version of the immersed boundary method. Tese de Doutorado, New York University. Citado na pág. 25, 26, 36, 41, 45, 51, 52, 58

Roma et al.(1999) A.M. Roma, C.S. Peskin e M.J. Berger. An adaptive version of the immersed boundary method. Journal of computational physics, 153(2):509-534. Citado na pág. 1, 4, 21

Shampine e Gear(1979) L.F. Shampine e C.W. Gear. A user's view of solving stiff ordinary differential equations. SIAM review, páginas 1-17. Citado na pág. 105

Sirignano(1999) WA Sirignano. Fluid dynamics and transport of droplets and sprays. Cambridge Univ Pr. Citado na pág. 3, 18, 77

Smooke et al.(1996) MD Smooke, A. Ern, MA Tanoff, BA Valdati, RK Mohammed, DF Marran e MB Long. Computational and experimental study of no in an axisymmetric laminar diffusion flame. Em Symposium (International) on Combustion, volume 26, páginas 2161-2170. Elsevier. Citado na pág. $65,83,84,85,86$ 
Stårner e Masri(2003) S. H. Stårner e A. R. Masri. An experimental comparison of evaporating and non-evaporating sprays in a simple turbulent jet flow. Em 9th International Conference on Liquid Atomization and Spray Systems, Sorrento, Italy. Citado na pág. 4, 6, 7, 65, 77, 78

Stoer e Bulirsch(2002) J. Stoer e R. Bulirsch. Introduction to numerical analysis, volume 12. Springer Verlag. Citado na pág. 40, 57, 105

Strikwerda(2004) J.C. Strikwerda. Finite difference schemes and partial differential equations. Society for Industrial Mathematics. Citado na pág. 25, 30, 32, 60

Temam(1968) R. Temam. Une méthode d'approximation de la solution des équations de navierstokes. Bull. Soc. Math. France, 98:115-152. Citado na pág. 25, 26

Thomas(1995) J.W. Thomas. Numerical partial differential equations: finite difference methods, volume 22. Springer Verlag. Citado na pág. 38

Trottenberg et al.(2001) U. Trottenberg, C. W. Oosterlee e A. Schüller. Multigrid. Academic Pr. Citado na pág. 25, 31, 32, 33, 35, 52, 60

Turns(1996) S. R. Turns. An introduction to combustion: concepts and applications. Citado na pág. $3,7,8,9,10,11,12,15,18,65,67,99,108$

Versteeg e Malalasekera(2007) H. K. Versteeg e W. Malalasekera. An introduction to computational fluid dynamics: the finite volume method. Prentice Hall. Citado na pág. 3, 7, 8, 9, 10, 11, 18, 32, 33, 99, 100, 101, 102

Veynante e Vervisch(2002) D. Veynante e L. Vervisch. Turbulent combustion modeling. Progress in Energy and Combustion Science, 28(3):193-266. Citado na pág. 101

Wang e Ruuth(2008) D. Wang e Steven J. Ruuth. Variable step-size implicit-explicit linear multistep methods for time-dependent partial differential equations. J. Comput. Math, 26(6): 835-855. Citado na pág. 1, 2, 25, 28

Zingale et al.(2009) M. Zingale, AS Almgren, JB Bell, A. Nonaka e SE Woosley. Low mach number modeling of type ia supernovae. iv. white dwarf convection. The Astrophysical Journal, 704:196. Citado na pág. 5 


\section{Índice Remissivo}

Acoplamento

entre as fases, 18, 20, 25, 40, 81

pressão-velocidade, 1

Bloco, 46, 48

discretizado, 4, 51, 70

celula

Célula computacional

fantasma, 75

Célula computacional, 4, 30-34, 39, 41, 48, 50,

$$
51,100
$$

fanstasma, 92

fantasma, 2, 45

visível, 77

vizinha, 82

Célulacomputacional, 75

Chama

de difusão, 84

Chama não pré-misturada, 3

Ordem de convergência, 57

Decomposição

de Hodge (Teorema), 26

de Reynolds, 99

Diâmetro

da gotícula, 19

Difusão artificial, 60

Discretização

espacial, 25

temporal, 25

Distribuição de probabilidade, 78

Domínio computacional, 30, 34, 39-41, 55

Energia, 12, 14

absoluta, 11

cinética, 10

sensível, 11

total, 10,11

Energia cinética turbulenta, 72

Entalpia

absoluta, 10, 11

de formação, 10, 16 sensível, 10, 11

total, 10

Equação

adimensional, 12, 111

da conservação da energia, 10, 107

da conservação da espécie química, 7, 112

da conservação de energia, 7

da conservação de massa, 7-9, 11, 15, 112

da quantidade de movimento linear, 7, 8, 14, 15,112

da temperatura, 113

de advecção-difusão, 55, 59

do estado termodinâmico, 7, 114

Escoamento

bifásico, 7

com baixo número de Mach, 4, 7, 13, 21, 60

incompressível, 3, 7, 13, 20, 58, 65

reativo, $7,13,83$

Valores característicos do, 12

Velocidade do, 9

Estratégia semi-implícita, 29, 30, 36

Fase

contínua, 3, 7, 18-21, 25, 40, 62

dispersa, 3, 6, 7, 18-21, 25, 40, 61, 62

líquida, 78

Formulação

euleriana, 3, 25, 40

lagrangiana, 3, 19, 25, 39, 40, 61

Fração

de mistura, 3, 7, 10, 12, 16, 59, 86, 103

mássica, 8, 9, 15-17, 84

Gotícula, 3, 49-51, 79

inerte, $7,18-21,45,77$

lagrangiana, 78

Jato

laminar não-reativo, 65

turbulento não-reativo, $65,69,77$

Malha, 99

base, 46 
bloco-estruturada, 4, 25, 30, 42, 45, 46, 48- Razão de refinamento, 45, 57

$51,53,68,70,75$

Cartesiana, 25, 31, 50

composta, 55

Euleriana, 39

uniforme, 30, 35, 41, 53, 68

Região de segurança, 50, 51

Método

\section{Multigrid, 25}

Multigrid-Multinível, 33

da projeção, 1, 25, 27

da projeção estendido, 4, 25, 60

de diferenças finitas, 4, 25, 31, 32

de Euler Modificado, 25, 40, 62

implícito-explícito, 1, 4, 25, 28

Método

Multigrid-Multinível, 52

Mistura

pobre, 17

rica, 17

Nível

base, 48,52

de refinamento, $45-50$

de Multigrid, 75

físico, 34

virtual, 34

Normas, 55

Número

adimensional, 12

de Lewis, 16, 83, 109

de Mach, 4, 7, 12-15, 22, 25-28, 33, 35, 113,

114

de Péclet, 32

de Prandtl, 12, 84, 114

de Reynolds, 12, 68, 78, 99, 112

de Schimdt turbulento, 101

de Schmidt, 12, 112

de Stokes, 18

Operador

de interpolação, 31, 32, 40

de prolongamento, 34

de restrição, 34

de suavização, 34

de espalhamento, 40

Operadores

diferenciais, 4, 31

diferenciais discretos, 31

Passo de integração, 4

Propriedades

adimensionais, 13, 111
Refinamento adaptativo, 1, 4, 70

critérios, 70

dinâmico, 45

estático, 45, 49

Refinamento adaptativo, 30

Refinamento adaptativo de malhas, 45 critérios, 50

\section{Sistema}

de equações diferenciais ordinárias, 3,10

linear, 2

rígido, 9

Solução Manufaturada, 58

Turbulência, 1, 99

simulação numérica direta, 5

Verificação numérica, 55 